\title{
Robotics-assisted treadmill exercise for cardiovascular rehabilitation early after stroke
}

Citation for published version (APA):

Stoller, O. (2015). Robotics-assisted treadmill exercise for cardiovascular rehabilitation early after stroke. [Doctoral Thesis, Maastricht University]. Datawyse / Universitaire Pers Maastricht. https://doi.org/10.26481/dis.20150513os

Document status and date:

Published: 01/01/2015

DOI:

10.26481/dis.20150513os

Document Version:

Publisher's PDF, also known as Version of record

\section{Please check the document version of this publication:}

- A submitted manuscript is the version of the article upon submission and before peer-review. There can be important differences between the submitted version and the official published version of record.

People interested in the research are advised to contact the author for the final version of the publication, or visit the DOI to the publisher's website.

- The final author version and the galley proof are versions of the publication after peer review.

- The final published version features the final layout of the paper including the volume, issue and page numbers.

Link to publication

\footnotetext{
General rights rights.

- You may freely distribute the URL identifying the publication in the public portal. please follow below link for the End User Agreement:

www.umlib.nl/taverne-license

Take down policy

If you believe that this document breaches copyright please contact us at:

repository@maastrichtuniversity.nl

providing details and we will investigate your claim.
}

Copyright and moral rights for the publications made accessible in the public portal are retained by the authors and/or other copyright owners and it is a condition of accessing publications that users recognise and abide by the legal requirements associated with these

- Users may download and print one copy of any publication from the public portal for the purpose of private study or research.

- You may not further distribute the material or use it for any profit-making activity or commercial gain

If the publication is distributed under the terms of Article $25 \mathrm{fa}$ of the Dutch Copyright Act, indicated by the "Taverne" license above, 


\section{Robotics-assisted treadmill exercise for cardiovascular rehabilitation early after stroke}


(c) Oliver Stoller 2015

All rights reserved. No part of this thesis may be reproduced or transmitted in any form or by any means, electronic or mechanical, including photocopying, recording or any information storage or retrieval system without permission in writing from the author, or, when appropriate from the publisher of the publications.

Published by Datawyse / Universitaire Pers Maastricht

ISBN 978-94-6159-429-7 


\title{
Robotics-assisted treadmill exercise for cardiovascular rehabilitation early after stroke
}

\author{
DISSERTATION \\ to obtain the degree of Doctor at Maastricht University, \\ on the authority of the Rector Magnificus, Prof. dr. L.L.G. Soete, \\ in accordance with the decision of the Board of Deans, \\ to be defended in public on \\ Wednesday $13^{\text {th }}$ May 2015 at 14:00 hours
}

Oliver Stoller

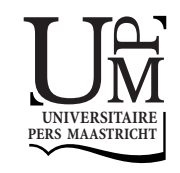




\section{Supervisor}

Prof. dr. R. A. de Bie

\section{Co-supervisors}

Prof. dr. K. J. Hunt, Bern University of Applied Sciences, Switzerland

PD. dr. E. D. de Bruin, Swiss Federal Institute of Technology Zurich, Switzerland

\section{Assessment Committee}

Prof. dr. M. H. Prins (chairman)

Prof. dr. R. Gassert, Swiss Federal Institute of Technology Zurich, Switzerland

Dr. K. Meijer

Prof. dr. R. Riener, Swiss Federal Institute of Technology Zurich, Switzerland

Prof. dr. L. W. van Rhijn

The research presented in this dissertation was conducted at the Institute for Rehabilitation and Performance Technology, Department of Engineering and Information Technology, of Bern University of Applied Sciences, Switzerland, and at the School for Public Health and Primary Care: CAPHRI, Department of Epidemiology, of Maastricht University, the Netherlands. The research project was funded by the Bern University of Applied Sciences. All clinical studies were conducted at the Reha Rheinfelden, Switzerland. 
For Ela 



\section{Contents}

1 General introduction $\quad 1$

2 Effects of cardiovascular exercise early after stroke: systematic review and metaanalysis

3 Evaluation of exercise capacity after severe stroke using robotics-assisted treadmill exercise: a proof-of-concept study

4 Feedback-controlled robotics-assisted treadmill exercise to assess and influence aerobic capacity early after stroke: a proof-of-concept study

5 Cardiopulmonary exercise testing early after stroke using feedback-controlled robotics-assisted treadmill exercise: test-retest reliability and repeatability

6 Cardiovascular rehabilitation soon after stroke using feedback-controlled roboticsassisted treadmill exercise: study protocol of a randomised controlled pilot trial

7 Efficacy of feedback-controlled robotics-assisted treadmill exercise to improve cardiovascular fitness early after stroke: a randomised controlled pilot trial

8 General discussion

Summary / Zusammenfassung

Valorisation

Acknowledgment

About the author 


\section{Abbreviations}

\begin{tabular}{ll} 
10MWT & 10 Meter Walk Test \\
6MWT & 6 Minute Walk Test \\
ACSM & American College of Sports Medicine \\
AT & Anaerobic threshold \\
BP & Blood pressure \\
BWS & Body weight support \\
BWSmin & Minimal body weight support \\
BWSTT & Bodyweight supported treadmill training \\
CI & Confidence interval \\
CLT & Constant load testing \\
CMSA & Chedoke-McMaster Stroke Assessment \\
CoV & Coefficient of variation \\
CPET & Cardiopulmonary exercise testing \\
EBI & Extended Barthel Index \\
ES & Effect size \\
FAC & Functional Ambulation Classification \\
FAI & Frenchay Activities Index \\
FC-RATE & Feedback-controlled robotics-assisted treadmill exercise \\
FIM & Functional Independence Measure \\
GET & Gas exchange threshold \\
GF & Guidance force \\
HR & Heart rate \\
HRpeak & Peak heart rate \\
HRR & Heart rate reserve \\
ICC & Intraclass correlation coefficient \\
IET & Incremental exercise testing \\
LoA & Limits of agreement \\
LT & Lactate threshold \\
MD & Mence \\
MMSE & Minilal State Examination \\
\hline
\end{tabular}




\begin{tabular}{|c|c|}
\hline MVC-W & Maximal voluntary contraction during walking \\
\hline $\mathrm{O}_{2}$ pulse & Oxygen pulse \\
\hline $\mathrm{P}$ & Power output, Work rate \\
\hline $\mathrm{P}_{\text {mech }}^{*}$ & Target mechanical work rate \\
\hline $\mathrm{P}_{\mathrm{ET}} \mathrm{CO}_{2}$ & End tidal fraction of $\mathrm{CO}_{2}$ \\
\hline $\mathrm{P}_{\mathrm{ET}} \mathrm{O}_{2}$ & End tidal fraction of $\mathrm{O}_{2}$ \\
\hline Pmax & Maximal work rate \\
\hline$P_{\text {mech }}$ & Mechanical work rate \\
\hline Ppeak & Peak work rate \\
\hline RATE & Robotics-assisted treadmill exercise \\
\hline RCP & Respiratory compensation point \\
\hline RER & Respiratory exchange ratio \\
\hline RERpeak & Peak respiratory exchange ratio \\
\hline $\mathrm{R}_{\mathrm{f}}$ & Respiratory rate \\
\hline $\mathrm{R}_{\mathrm{fpeak}}$ & Peak respiratory rate \\
\hline $\mathrm{RMSE}_{\mathrm{P}}$ & Root mean square error of work rate tracking \\
\hline RPE & Rate of perceived exertion \\
\hline SD & Standard deviation \\
\hline SDdiff & Standard deviation of the difference \\
\hline SE & Standard error \\
\hline SEM & Standard error of the measurement \\
\hline SIS & Stroke Impact Scale \\
\hline SMD & Standardised mean difference \\
\hline$\dot{\mathrm{V} C O}_{2}$ & Carbon dioxide output \\
\hline$\dot{\mathrm{V}}_{\mathrm{E}}$ & Ventilation rate \\
\hline$\dot{\mathrm{V}}_{\text {Epeak }}$ & Peak ventilation rate \\
\hline $\mathrm{V}_{2}$ & Oxygen uptake \\
\hline$\dot{\mathrm{V}} \mathrm{O}_{2} \max$ & Maximal oxygen uptake \\
\hline$\dot{\mathrm{V}} \mathrm{O}_{2}$ peak & Peak oxygen uptake \\
\hline$\dot{\mathrm{V}}_{\mathrm{T}}$ & Tidal volume \\
\hline VT & Ventilatory threshold \\
\hline
\end{tabular}



Chapter 1

\section{General introduction}


The human species is designed to perform prolonged physical exercise. Our ability to overcome long distances is not just a biologically aberrant behaviour, human cardiovascular capabilities have deep evolutionary roots [1].

In the middle Palaeolithic age, 200,000 years ago, hunter-gatherers profited from a welldeveloped cardiovascular capacity to scavenge [2]. Even with the invention of better projectile weapons such as the bow and arrow, which was invented within the last 50,000 years, chasing prey for several hours required a superior cardiovascular capacity to meet nutritional demands [3]. In addition, the human race spent ages with mass migrations, which was associated with long walks through rough terrain [4]. The extended wanderings by foot combined with physical challenges to secure basic needs required good physical fitness levels for all tribe members.

Unsurprisingly, cardiovascular exercise might be responsible for several health-conserving factors [5]. However, individuals living in western countries do not reach recommended physical activity levels, and cardiovascular related diseases, such as stroke, are rising [6]. Demographic changes and medical progress will inevitably increase the number of individuals suffering from constrained immobilisation after serious healthcare related events [7]. This will indubitably lead to future challenges in preventive and rehabilitative medicine.

Considering the deep evolutionary roots of cardiovascular exercise and the set of health-related issues associated with physical inactivity, there is a need for advanced intervention strategies to facilitate cardiovascular exercise even in severely impaired populations such as early after stroke. Here, rehabilitation robotics might have the potential to provide relevant support.

This chapter provides an overview of: (1) the current knowledge on cardiovascular exercise and health, (2) the state-of-the-art in the assessment of exercise capacity and its interpretation, (3) the recent standards in stroke rehabilitation with a focus on cardiovascular health, and (4) the role of rehabilitation robotics. Finally, the hypotheses and the aims, followed by the outline of this thesis, are presented. 


\section{Cardiovascular exercise and health}

Over recent decades, numerous long-term prospective follow-up studies reinforced scientific evidence that regular cardiovascular exercise can maintain and improve overall health status. The results of several expert panels and scientific statements clearly reveal that individuals who are active tend to live longer and have lower risk for cardiovascular disease, thromboembolic stroke, hypertension, type 2 diabetes mellitus, osteoporosis, obesity, colon cancer, breast cancer, anxiety and depression $[5,8,9,10,11,12,13,14]$. There is a direct relationship between cardiovascular fitness and mortality, whereby the level of fitness has been shown to be a more important predictor of death than established risk factors such as smoking, high blood pressure, high cholesterol, and diabetes [15]. As a result, tremendous global efforts have been set up to promote regular cardiovascular exercise to positively modify risk factor profiles in apparently healthy individuals as well as in various diseases $[16,17]$.

\section{Effects of cardiovascular exercise}

Regular cardiovascular exercise leads to significant improvements in functional capacity. Cardiovascular exercise increases oxygen uptake $\left(\mathrm{VO}_{2}\right)$ by increasing both cardiac output and the ability of muscles to extract and use oxygen from the blood. This fact directly results in decreased oxygen demand for the same level of external work, thus leading to an improvement in exercise capacity. Several physiological changes in cardiovascular, musculoskeletal, and neurological function are directly responsible for the improvement in overall exercise capacity, and have an indirect effect on a number of risk factors for mortality.

In more detail, cardiovascular exercise causes a downregulation of beta-adrenergic receptors which decreases the resting heart rate [18], whereby a reduction of overall blood pressure allows an increase in ejection fraction and stroke volume due to a reduction of left ventricle afterloading [19]. An increase in myocardial contractility boosts the oxygen supply of the myocardium, further enhances cardiac stroke volume, and leads to ventricular hypertrophy. In addition, emerging evidence suggests that cardiovascular exercise improves endothelium function $[20,21]$.

Regular cardiovascular exercise increases the storage of fats and carbohydrates within the muscles, allowing for increased endurance [22]. The neovascularisation of the muscle sarcomeres enhances blood flow to the muscle cells, whereby the speed at which aerobic metabolism activates rises $[23,24]$. Both aspects directly enhance exercise capacity and lead to a shorter recovery phase, thus increasing the ability for early exercise readmission. 
Cardiovascular exercise enhances blood flow to the central neuronal system which can accelerate central processes [25]. At a neuronal level, mild stress generated by exercise promotes neurogenesis [26]. As a result, the number of dendrite connections will increase, which leads to a denser and more effective network. Cardiovascular exercise has therefore been associated with cognitive functions such as attention, processing speed, executive function, and memory $[27,28,29,30,31,32]$.

Regarding the effects on risk factors, hypertension can be prevented and well controlled by regular cardiovascular exercise [33, 34]. Several studies demonstrated a decrease in high blood pressure profile, which was sustained even after reduction in hypertensive medication $[35,36,37$, 38]. Regular exercise also has beneficial effects on diabetes mellitus. The mechanisms behind this are a decrease in glucose production by the liver, an increased number of muscle cells that utilise more glucose than adipose tissue, and an increased sensitivity to insulin [39, 40, 41, 42]. Furthermore, emerging evidence suggests that cardiovascular exercise favourably affects coagulation and haemostatic factors [43], which are important pathophysiological mechanisms of thromboembolic stroke and coronary syndromes [44]. In addition, regular cardiovascular exercise affects blood lipid profiles and is an important contributor to weight loss [45].

\section{Cardiovascular exercise prescription}

Cardiovascular exercise requires a prescription, with consideration of appropriate dosage and possible side effects [46]. Physical activities that demand a strong increase in cardiovascular stress by including dynamic exercise of large muscle groups should be considered. Exercise should be prescribed 3 to 6 times per week for a minimum of 30 minutes per session at a minimum intensity of $40 \%$ to $60 \%$ of the maximal exercise capacity [13, 47]. A useful approach to control the intensity is to identify the desirable heart rate, the corresponding rate of perceived exertion (Borg scale) [48], or the direct $\dot{\mathrm{V}} \mathrm{O}_{2}$.

\section{Risks of cardiovascular exercise}

Three of the most important risk factors for cardiovascular exercise are age, presence of cardiac disease, and exercise intensity [47]. There is evidence that heavy exertion may increase the risk of acute myocardial infarction by a factor of 4 [49]. However, the more active the individuals are, the lower is the risk for development of a myocardial infarction $[50,51]$. Importantly, the risk for musculoskeletal injuries increases as the intensity, the amount, and the physical impact of exercise rises [52]. 


\section{Exercise testing and interpretation}

The ability to perform physical exercise is critically related to the cardiovascular systems capacity to supply oxygen $\left(\mathrm{O}_{2}\right)$ to the muscles and to clear carbon dioxide $\left(\mathrm{CO}_{2}\right)$ from the blood via the lungs. While $\dot{\mathrm{VO}}_{2}$ is tightly coupled to external power output (P) due to energy demands in the muscle cells, maximal oxygen uptake $\left(\dot{\mathrm{VO}}_{2} \max \right)$ has been accepted as the gold standard for assessment of exercise capacity. Cardiopulmonary exercise testing (CPET) has proven to be an important diagnostic tool. The application of CPET evaluates an individuals aerobic capacity by assessing cardiopulmonary performance parameters based on the gas exchange, ventilation, and heart rate during physical exercise.

\section{Cardiopulmonary exercise testing}

Two modes of exercise are commonly employed for CPET: treadmill and leg cycle ergometry, whereby treadmill exercise testing has some advantages over leg cycle ergometry. Most individuals are more familiar with walking than cycling and walking/running permits a larger muscle mass to be recruited and more work to be done against gravity leading to a 5-10\% higher $\dot{\mathrm{V}} \mathrm{O}_{2}$ max compared to leg cycle ergometry $[53,54,55,56]$. However, walking on the treadmill requires superior motor skills and is therefore not the primary choice in disabled populations. Furthermore, the quantification of external work rate during treadmill exercise is a difficulty. In contrast, leg cycle ergometry is less prone to introduce movement artefacts into measurements and the guidance of external work rate can be easily done by increasing the resistance to pedalling.

The most common concepts are progressive incremental ramp protocols and constant workload protocols, but some mixed methods have also been implemented. An efficient incremental exercise testing protocol (IET) on a leg cycle ergometer consists of at least 3 minutes of rest, followed by 3 minutes of unloaded pedalling followed by the incremental phase of exercise ( 5 to 25 $\mathrm{W} /$ minute) until the subject reaches volitional exhaustion or termination criteria are reached. If a treadmill is used, the work rate can be incremented at regular intervals with a combination of speed and grade. Constant load testing (CLT) consists of at least 3 minutes of rest, followed by an unloaded warm up phase of 3 minutes followed by at least 5 minutes of continuous exercise.

\section{Outcome variables}

The main focus of CPET is on maximal exercise capacity and on certain thresholds that indicate major changes in the metabolic state. A plateau in the $\dot{\mathrm{V}} \mathrm{O}_{2}-\mathrm{P}$ relation during incremental exercise 
has been defined as the best indication that $\dot{\mathrm{VO}}_{2}$ max has been reached. It is defined as the highest $\mathrm{O}_{2}$ consumption while performing dynamic exercise involving a large part of total muscle mass [47]. However, reaching a clear plateau is not always achieved [57, 58]. Consequently, the term $\dot{\mathrm{V}} \mathrm{O}_{2}$ peak is used instead of $\dot{\mathrm{V}} \mathrm{O}_{2}$ max. Healthy middle-aged individuals have $\dot{\mathrm{VO}_{2}}$ peak values of $41.3 \pm 9.2 \mathrm{~mL} / \mathrm{min} / \mathrm{kg}$ [59].

Another important variable of exercise capacity is external power output $(\mathrm{P})$, also known as external work rate. $\mathrm{P}$ strongly depends on the choice of the device. As a reference during leg cycle exercise testing, healthy middle-aged individuals will reach $\mathrm{P}$ values (Ppeak) up to $300 \mathrm{~W}$ [60], where the oxygen cost of work $\left(\Delta \dot{\mathrm{VO}}_{2} / \Delta \mathrm{P}\right)$ is normally about $8.5-11 \mathrm{~mL} / \mathrm{min} / \mathrm{W}$ [61].

Important thresholds during CPET include the first and the second anaerobic threshold (AT) [62]. While $\dot{\mathrm{VCO}}_{2}$ increases nearly as much as $\dot{\mathrm{VO}}{ }_{2}$ does over the lower work rate range, a sharp increase towards higher work rates reflects the buffering of lactic acid and defines the first anaerobic threshold, also known a the gas exchange threshold (GET), ventilatory threshold (VT), or lactate threshold (LT). In sedentary individuals, the first AT occurs at about 50-70\% of $\dot{\mathrm{VO}}_{2}$ peak $[63,64]$. With further increase in exercise intensity, the muscular lactate production rate then exceeds the systemic lactate buffering rate, which causes hyperventilation and defines the second AT, also known as the respiratory compensation point (RCP). A rough index of metabolic events is the ratio of $\dot{\mathrm{VCO}_{2}} / \mathrm{VO}_{2}$ that is called the respiratory exchange ratio (RER). An RER greater than 1.0 indicates the rise of $\dot{\mathrm{V} C O}{ }_{2}$ derived from lactic acid or by hyperventilation. However, further indices such as the ventilatory equivalents for $\dot{\mathrm{VO}}_{2}$ and $\dot{\mathrm{VCO}}_{2}$ need to be consulted to define the AT accurately, and ventilation rate $\left(\dot{\mathrm{V}}_{\mathrm{E}}\right)$ versus $\dot{\mathrm{VCO}}_{2}$ slope and the end tidal fraction of $\mathrm{O}_{2}\left(\mathrm{P}_{\mathrm{ET}} \mathrm{O}_{2}\right)$ and $\dot{\mathrm{V} C O} \mathrm{P}_{2}\left(\mathrm{P}_{\mathrm{ET}} \mathrm{CO}_{2}\right)$ are needed to estimate the RCP.

The most common ventilatory variables include changes in $\dot{V}_{\mathrm{E}}$ and breathing pattern as characterised by respiratory frequency $\left(\mathrm{R}_{\mathrm{f}}\right)$ and tidal volume $\left(\dot{\mathrm{V}}_{\mathrm{T}}\right)$. The rise in $\dot{\mathrm{V}}_{\mathrm{E}}$ with exercise is associated with an increase in both depth and frequency of breathing. As exercise progresses, both $\dot{V}_{\mathrm{T}}$ and $\mathrm{R}_{\mathrm{f}}$ increase until 70 to $80 \%$ of peak exercise; thereafter $\mathrm{R}_{\mathrm{f}}$ predominates $[65,66]$.

The achievement of age-predicted values for maximal heart rate during exercise is often used as a reflection of maximal or peak effort and indices for $\dot{\mathrm{VO}_{2}}$ peak. However, the use of this marker as a strict exercise end point is not recommended [46]. Further analyses such as the heart rate- $\dot{\mathrm{V}} \mathrm{O}_{2}$ relationship $\left(\mathrm{O}_{2}\right.$ pulse $)$ and the heart rate response provide indirect measurements for cardiac output and might be used as index of cardiac function during exercise. 


\section{Exercise limitations}

In healthy individuals, exercise capacity appears to be limited by $\mathrm{O}_{2}$ delivery, the product of cardiac output and arterial $\mathrm{O}_{2}$ content. Due to the fact that arterial $\mathrm{O}_{2}$ content is normally maintained even at peak exercise, cardiac output is likely the limiting factor [67]. Adding other exercising muscles to the two-legged exercise does not increase $\dot{\mathrm{VO}_{2}}$ peak, suggesting that $\mathrm{O}_{2}$ blood flow (cardiac output) has reached its maximal capacity. In addition, the diffusive capacity of $\mathrm{O}_{2}$ at muscle tissue level is another limiting factor. The movement of $\mathrm{O}_{2}$ from the capillaries to the mitochondria (tissue diffusion) is directly related to the pressure gradient, which does not increase with progressive exercise. As a result, only improved tissue capillarisation could further increase the $\mathrm{O}_{2}$ delivery of the muscle cells.

\section{Safety}

CPET is a relatively safe procedure. In a survey of 1,375 clinical exercise testing facilities, the risk of dying during CPET was 0.5 per 10,000 tests [68]. However, several absolute and relative contraindications have to be considered. Among these are unstable angina, syncope, uncontrolled heart failure or systemic hypertension, the presence of serious cardiac dysrhythmias on the resting electrocardiogram, or mental impairment leading to inability to cooperate [46]. Furthermore, individuals who are unable to exercise because of neurological or orthopaedic problems are not candidates for conventional CPET procedures.

\section{CPET in disabled populations}

CPET is able to determine the level of functional disability from various diseases because it provides an objective assessment of exercise capacity. For example, exercise testing after myocardial infarction is well established and yields information regarding risk stratification, prognosis, and functional capacity for activity prescription [47]. Furthermore, CPET can be used to evaluate disability and age-associated changes in exercise capacity and prevalence and severity of disease status. Special protocols are available for disabled individuals with stroke, lower limb amputation or spinal cord injury. Advanced testing protocols use semi-recumbent cycle ergometry, arm cycle ergometry, or combined upper- and lower-limb ergometry to overcome mobility and stability issues. 


\section{Stroke}

Each year about 16 million people worldwide experience a first-ever stroke. This number is expected to rise to 23 million first-ever strokes in 2030 [69]. Globally, stroke is the second leading cause of death above the age of 60 years, and the fifth leading cause of death in people aged 15 to 59 years old [70].

\section{Recovery after stroke}

The primary goals after stroke are to reduce brain injury and promote maximal recovery. Rapid detection and appropriate emergency medical care are essential in the acute phase (0-7 days poststroke) [71]. Once a stroke survivor is medically stable, the focus shifts to rehabilitation. The goals of the subacute phase (7 days - 6 months post-stroke) include preventing secondary health complications, minimising impairments, and achieving functional goals that promote independence in activities of daily living [72].

About a third of stroke survivors recover almost completely or with only mild impairments, whereas around two-thirds experience moderate to severe impairments requiring special care such as inpatient rehabilitation and/or a long-term care facility [73, 74].

The early and spontaneous recovery after stroke is often attributed to resolution of oedema or return of circulation within the ischaemic area and may continue for up to 8 weeks $[75,76,77]$. The later recovery, based on neural plasticity and reorganisation, plays an important role in the restoration of function. Neural plasticity and reorganisation of the brain leads to functional changes in the surrounding brain tissue and in remote locations that have structural connections with the injured area $[78,79,80]$.

The major part of neurological recovery occurs within the first 3 months, and recovery may continue at a slower pace for at least 6-12 months [81]. Progress towards recovery may plateau at any stage of recovery with only a very small percentage achieving full recovery $[81,82,83]$. Recent clinical practice guidelines recommend that rehabilitation therapy should start as early as possible, once medical stability is achieved [72].

Higher intensity of therapy results in improved functional outcomes [84, 85, 86, 87, 88]. Therefore, the delivery of high intensity therapy should be the priority. In reality, the amount of therapy stroke survivors get is often inadequate because of a lack of prioritisation and difficult implementation [89].

Repetition plays a major role in inducing and maintaining changes in the brain [90]. However, repetitive physical activity alone is not enough to produce increased motor cortical representations 
[78]. Instead, an element of skilled motor learning is required, in addition to repetition, for cortical reorganisation/plasticity to occur. Therefore, extensive task-specific therapy appears important to maximise recovery of function $[91,92]$.

\section{Cardiovascular status after stroke}

The majority of stroke survivors present with cardiovascular related health issues. Around $75 \%$ of post-stroke individuals exhibit pre-existing cardiac disease [93, 94]. It has been hypothesised that stroke survivors may be more disabled by associated cardiac disease than by the stroke incident itself [95]. Individuals after stroke frequently have significant atherosclerotic lesions throughout their vascular system and are at heightened risk for associated cardiovascular disease [93, 96]. Moreover, stroke survivors are often deconditioned and predisposed to a sedentary lifestyle due to their disability. This limits performance of physical activity and may contribute to a heightened risk for recurrent stroke and cardiovascular disease. CPET studies in stroke indicate that $\dot{\mathrm{V}} \mathrm{O}_{2}$ peak is reduced to $10-17 \mathrm{~mL} / \mathrm{min} / \mathrm{kg}$ within 0-30 days after the stroke, and does not rise above 20 $\mathrm{mL} / \mathrm{min} / \mathrm{kg}$ after six months $[97,98,99,100]$. These values are $25-60 \%$ lower than $\dot{\mathrm{VO}}_{2}$ peak in age-matched, healthy subjects [101, 102, 103]. This intolerance is likely due to several factors, including bed-rest-induced deconditioning, concomitant left ventricular dysfunction, and the associated severity of neurological involvement [104]. As a consequence, the early and persistent decline in exercise capacity can delay or inhibit participation in exercise programmes, complicate the rehabilitation process and long-term post-stroke course of care, and limit the ability of the individual to perform functional activities independently [94, 105].

\section{Cardiovascular rehabilitation after stroke}

The major cardiovascular rehabilitation goals after stroke are to prevent complications of prolonged immobilisation, decrease recurrent stroke and cardiovascular events, and increase exercise capacity [104]. At first, simple exposure to orthostatic or gravitational stress has been shown to obviate much of the deterioration in exercise tolerance [106]. Secondly, a reduction of risk factors can decrease the incidence of recurrent strokes and coronary events while cardiovascular exercise programme can enhance glucose regulation and promote decreases in body weight and fat stores, blood pressure (particularly in hypertensive patients), total blood cholesterol, serum triglycerides, and low-density lipoprotein cholesterol [107]. 
The third rehabilitation goal should be the improvement of exercise capacity. Evidence suggests that cardiovascular exercise training improves aerobic capacity, walking endurance, and walking speed in mildly to moderately impaired stroke survivors [108].

\section{Barriers for cardiovascular rehabilitation after stroke}

The combination of comorbidities and neurological deficits that are unique to each stroke survivor requires an individual approach to safely and effectively implement cardiovascular rehabilitation programmes. Common methods (i.e. treadmill exercise, cycle ergometry) are mainly designed for moderate to mildly impaired individuals, and present unique challenges in severely motor impaired stroke survivors due to limited motor control (non-ambulatory status, limited trunk control), poor postural control, uncoordinated control of the affected limbs, cardiac risk factors, and inadequate cognitive function. As a result, the implementation of cardiovascular rehabilitation after stroke appears to be a major challenge. Therefore, advanced concepts are needed to assess exercise capacity and facilitate cardiovascular exercise in severely motor impaired individuals post-stroke. Technological achievements in rehabilitation robotics might provide solutions for this problem.

\section{Rehabilitation robotics}

Technological progress has enabled several human-machine interfaces to move into various aspects of daily life. Great strides have been made in the design and control of wearable robots (i.e. powered exoskeletons) to provide mobility and to further understand motor learning and recovery. Advances in control strategies, power supplies, and actuators have led to high-quality devices that have potential for rehabilitation purposes. For example, powered exoskeletons can provide prolonged exercise sessions and accurate evaluation of therapy outcomes. This opens new perspectives for the control and guidance of exercise intensity during stroke rehabilitation.

\section{Rationale for rehabilitation robotics in stroke}

A stroke mostly results in sudden impairments of the motor system, which leads to severe disability and major restrictions in rehabilitation endeavours and in daily life. Hemiparesis of the extremities leads to severe impairments of physical functioning such as walking, stair climbing, or grasping. Here, rehabilitation robotics might have the potential to assist or fully support the individual during desired movements, whereby sophisticated control strategies might assist the individual only as needed, which would additionally facilitate recovery of motor function and exercise capacity. 


\section{State of the art}

Several robotic devices have been developed to support and facilitate motor recovery. To date, devices for the upper extremities are designed for stationary practice in a sitting position (MIME [109], MIT-Manus [110], Bi-Manu-Track [111], NeReBot [112], ARMin [113], ARM Guide [114], REHA ROB [115], ARMOR [116], T-WREX [117]). Robotic devices for the lower extremities include systems for enabling treadmill training (Lokomat [118], LokoHelp [119], ARTHur [120], POGO \& PAM [121], ALEX [122], LOPES [123]), stair climbing (G-EO [124]), or stationary walking (MotionMaker [125]). In addition, some lower extremity devices enable overground walking (KineAssist [126], WalkTrainer [127], ReWalk [128], HAL [129]). Recent research suggests that robotics-assisted therapy is an effective complement to conventional rehabilitation, but there is no clear evidence that robotics-assisted training is superior. A meta-analysis on upper extremity robotic training after stroke yielded small but significant improvements in motor recovery and favours robotics-assistance compared to conventional care [130]. Similar to the lower extremities, a recent meta-analysis concluded that individuals who receive robotics-assisted gait training after stroke are more likely to achieve independent walking than individuals who receive gait training without these devices [131]. Importantly, individuals early after stroke (within 3 months) and individuals with severe impairments seem to benefit most from this type of intervention.

\section{Potential for cardiovascular exercise testing and prescription}

While robotic devices have been primarily developed to facilitate motor recovery, a hidden aspect might be the potential for cardiovascular exercise in severely disabled populations. The implementation of effective protocols to improve exercise capacity would provide a complement to the application of these devices for neurological rehabilitation, thus expanding the field of application and clinical uptake. Initial work on robotics-assisted treadmill exercise using the Lokomat [118] has shown that walking within a powered exoskeleton is not entirely passive [132]. Even a subject with motor-complete tetraplegia shows a metabolic response by increasing oxygen uptake, ventilation rate, and heart rate [133]. Intervention studies on robotics-assisted treadmill exercise have shown improvements in left ventricular function, coronary flow reserve, and endothelial dysfunction after incomplete spinal cord injury [134]. In addition, the implementation of feedback-control structures to guide voluntary participation during robotics-assisted treadmill exercise led to promising results for cardiovascular rehabilitation $[135,136]$. 


\section{Hypotheses and aims of the thesis}

Considering the impact of regular cardiovascular exercise on overall health status, risk factor modification for cardiovascular disease, and improvement of exercise capacity, a careful implementation into stroke rehabilitation has a lot of potential. Moreover, the effects on the vascular, neural, and muscular systems lead to the hypothesis that cardiovascular exercise training might facilitate neuroplasticity (i.e. motor recovery, cognitive function). To date, rehabilitation strategies to improve cardiovascular fitness in severely motor impaired individuals after stroke are inefficient and do not embody principles for motor learning (i.e. task-specific exercise). Considering the relatively short intervention window during stroke recovery, advanced intervention approaches (i.e. rehabilitation robotics) might have high potential to facilitate a wide spectrum of key elements (e.g. cardiovascular stress, task-specific training) for highly effective stroke rehabilitation.

The driving hypothesis of the following thesis is that the implementation of cardiovascular exercise training and assessment early after stroke will positively influence cardiovascular health, which could facilitate overall recovery after stroke. Robotic technology (e.g. robotics-assisted treadmill exercise) might have the potential to introduce cardiovascular rehabilitation into the early stages after stroke, where deconditioning and severe motor impairments are highly prevalent. This novel concept would provide a relevant methodology for exercise testing and cardiovascular exercise prescription for stroke survivors with severe motor limitations.

The aims of this thesis are: (1) to review the knowledge about the effects of cardiovascular exercise early after stroke, (2) to implement a robotic-based strategy to accomplish recent standards for exercise testing and prescription for severely motor impaired stroke survivors, and (3) to systematically evaluate the concept within a clinical setting. 


\section{Outline of the thesis}

This thesis focuses on the implementation of cardiovascular exercise early after stroke. In CHAPTER 2, a systematic review of the literature about the state of evidence for cardiovascular exercise early after stroke is presented. A meta-analysis of 11 controlled trials was performed to evaluate the benefits of early cardiovascular intervention approaches and to reveal potential gaps in knowledge. CHAPTER 3 presents first clinical experimental results of robotics-assisted treadmill technology for assessment of exercise capacity and guidance of exercise intensity in severely motor impaired stroke survivors. Furthermore, a feedback-control mechanism has been implemented to increase the exercise intensity during robotics-assisted treadmill exercise, which has been tested in a further clinical trial (CHAPTER 4). Thereby, force measurements from the exoskeleton were used to guide active participation during robotics-assisted walking. CHAPTER 5 investigates test-retest reliability and repeatability of the feedback-control concept to evaluate exercise capacity. Severely motor impaired individuals early after stroke performed repeated CPET within a feedback-controlled robotics-assisted treadmill. Furthermore, a study protocol (CHAPTER 6) for a randomised controlled pilot trial is presented that aims to compare feedback-controlled roboticsassisted treadmill exercise with conventional robotics-assisted treadmill exercise in severely motor impaired individuals early after stroke. CHAPTER 7 presents the results of this longitudinal clinical intervention study. Finally, CHAPTER 8 provides an overall discussion and the main original contributions.

Since CHAPTERS 2-7 have been submitted or published as separate articles in international peer-reviewed scientific journals, there might be some overlap. 


\section{References}

[1] D. E. Lieberman and D. M. Bramble, "The evolution of marathon running : capabilities in humans," Sports Med, vol. 37, no. 4-5, pp. 288-90, 2007.

[2] D. M. Bramble and D. E. Lieberman, "Endurance running and the evolution of homo," Nature, vol. 432, no. 7015, pp. 345-52, 2004.

[3] J. J. Shea, “The origins of lithic projectile point technology: evidence from africa, the levant, and europe," Journal of Archaeological Science, vol. 33, no. 6, pp. 823-846, 2006.

[4] D. B. Goldstein and L. Chikhi, "Human migrations and population structure: What we know and why it matters," Annual Review of Genomics and Human Genetics, vol. 3, pp. 129-152, 2002.

[5] J. Myers, "Exercise and cardiovascular health," Circulation, vol. 107, no. 1, pp. E2-E5, 2003.

[6] W. L. Haskell, S. N. Blair, and J. O. Hill, "Physical activity: Health outcomes and importance for public health policy," Preventive Medicine, vol. 49, no. 4, pp. 280-282, 2009.

[7] G. Lanzieri, “The greying of the baby boomers," Statistics in focus, 2011.

[8] D. E. R. Warburton, C. W. Nicol, and S. S. D. Bredin, "Health benefits of physical activity: the evidence," Canadian medical association journal, vol. 174, no. 6, pp. 801-809, 2006.

[9] R. R. Pate, M. Pratt, S. N. Blair, W. L. Haskell, C. A. Macera, C. Bouchard, D. Buchner, W. Ettinger, G. W. Heath, A. C. King, A. Kriska, A. S. Leon, B. H. Marcus, J. Morris, R. S. Paffenbarger, K. Patrick, M. L. Pollock, J. M. Rippe, J. Sallis, and J. H. Wilmore, "Physical-activity and public-health - a recommendation from the centers-for-disease-control-and-prevention and the american-college-of-sports-medicine," Jama-Journal of the American Medical Association, vol. 273, no. 5, pp. 402-407, 1995.

[10] C. Bouchard, S. N. Blair, and W. L. Haskell, Physical activity and health, vol. 374. Human Kinetics Champaign, IL, 2007.

[11] L. Miles, "Physical activity and health," Nutrition Bulletin, vol. 32, no. 4, pp. 314-363, 2007.

[12] N. Cavill, S. Kahlmeier, and F. Racioppi, Physical activity and health in Europe: evidence for action. World Health Organization, 2006.

[13] W. L. Haskell, I. M. Lee, R. R. Pate, K. E. Powell, S. N. Blair, B. A. Franklin, C. A. Macera, G. W. Heath, P. D. Thompson, and A. Bauman, "Physical activity and public health - updated recommendation for adults from the american college of sports medicine and the american heart association," Circulation, vol. 116, no. 9, pp. 1081-1093, 2007.

[14] Y. K. Kesaniemi, J. Danforth, E., M. D. Jensen, P. G. Kopelman, P. Lefebvre, and B. A. Reeder, "Dose-response issues concerning physical activity and health: an evidence-based symposium," Med Sci Sports Exerc, vol. 33, no. 6 Suppl, pp. S351-8, 2001.

[15] J. Myers, M. Prakash, V. Froelicher, D. Do, S. Partington, and J. E. Atwood, "Exercise capacity and mortality among men referred for exercise testing," New England Journal of Medicine, vol. 346, no. 11, pp. 793-801, 2002.

[16] "A guide for population-based approaches to increasing levels of physical activity: implementation of the who global strategy on diet, physical activity and health," World Health Organization, 2007.

[17] "Global recommendations on physical activity for health.," World Health Organization, 2010.

[18] R. H. Fagard, "Physical activity, physical fitness and the incidence of hypertension," J Hypertens, vol. 23, no. 2, pp. 265-7, 2005. 
[19] R. J. Shephard and G. J. Balady, "Exercise as cardiovascular therapy," Circulation, vol. 99, no. 7, pp. 963-72, 1999.

[20] S. Charo, N. Gokce, and J. A. Vita, "Endothelial dysfunction and coronary risk reduction," J Cardiopulm Rehabil, vol. 18, no. 1, pp. 60-7, 1998.

[21] R. Hambrecht, A. Wolf, S. Gielen, A. Linke, J. Hofer, S. Erbs, N. Schoene, and G. Schuler, "Effect of exercise on coronary endothelial function in patients with coronary artery disease," $N$ Engl J Med, vol. 342, no. 7, pp. 454-60, 2000.

[22] W. D. McArdle, F. I. Katch, and V. L. Katch, Exercise physiology: nutrition, energy, and human performance. Lippincott Williams Wilkins, 2010.

[23] T. Gustafsson and W. E. Kraus, "Exercise-induced angiogenesis-related growth and transcription factors in skeletal muscle, and their modification in muscle pathology," Front Biosci, vol. 6, pp. D75-D89, 2001.

[24] M. Vogt, A. Puntschart, J. Geiser, C. Zuleger, R. Billeter, and H. Hoppeler, "Molecular adaptations in human skeletal muscle to endurance training under simulated hypoxic conditions," Journal of Applied Physiology, vol. 91, no. 1, pp. 173-182, 2001.

[25] N. Uysal, K. Tugyan, B. M. Kayatekin, O. Acikgoz, H. A. Bagriyanik, S. Gonenc, D. Ozdemir, I. Aksu, A. Topcu, and I. Semin, "The effects of regular aerobic exercise in adolescent period on hippocampal neuron density, apoptosis and spatial memory," Neurosci Lett, vol. 383, no. 3, pp. 241-5, 2005.

[26] M. P. Mattson, W. Duan, R. Wan, and Z. Guo, "Prophylactic activation of neuroprotective stress response pathways by dietary and behavioral manipulations," NeuroRx, vol. 1, no. 1, pp. 111-116, 2004.

[27] A. D. Brown, C. A. McMorris, R. S. Longman, R. Leigh, M. D. Hill, C. M. Friedenreich, and M. J. Poulin, "Effects of cardiorespiratory fitness and cerebral blood flow on cognitive outcomes in older women," Neurobiol Aging, vol. 31, no. 12, pp. 2047-57, 2010.

[28] M. Ploughman, "Exercise is brain food: The effects of physical activity on cognitive function," Developmental Neurorehabilitation, vol. 11, no. 3, pp. 236-240, 2008.

[29] J. Weuve, J. H. Kang, J. E. Manson, M. M. Breteler, J. H. Ware, and F. Grodstein, "Physical activity, including walking, and cognitive function in older women," JAMA, vol. 292, no. 12, pp. 1454-61, 2004.

[30] K. Yaffe, D. Barnes, M. Nevitt, L. Y. Lui, and K. Covinsky, "A prospective study of physical activity and cognitive decline in elderly women: women who walk," Arch Intern Med, vol. 161, no. 14, pp. 1703-8, 2001.

[31] J. R. Hughes, "Psychological effects of habitual aerobic exercise: A critical review," Preventive medicine, vol. 13, no. 1, pp. 66-78, 1984.

[32] P. J. Smith, J. A. Blumenthal, B. M. Hoffman, H. Cooper, T. A. Strauman, K. Welsh-Bohmer, J. N. Browndyke, and A. Sherwood, "Aerobic exercise and neurocognitive performance: a meta-analytic review of randomized controlled trials," Psychosom Med, vol. 72, no. 3, pp. 239-52, 2010.

[33] J. Paffenbarger, R. S., A. L. Wing, R. T. Hyde, and D. L. Jung, "Physical activity and incidence of hypertension in college alumni," Am J Epidemiol, vol. 117, no. 3, pp. 245-57, 1983.

[34] S. N. Blair, N. N. Goodyear, L. W. Gibbons, and K. H. Cooper, "Physical fitness and incidence of hypertension in healthy normotensive men and women," JAMA, vol. 252, no. 4, pp. 487-90, 1984.

[35] L. S. Pescatello, A. E. Fargo, J. Leach, C. N., and H. H. Scherzer, "Short-term effect of dynamic exercise on arterial blood pressure," Circulation, vol. 83, no. 5, pp. 1557-61, 1991.

[36] P. F. Kokkinos and V. Papademetriou, "Exercise and hypertension," Coron Artery Dis, vol. 11, no. 2, pp. 99102, 2000. 
[37] R. H. Fagard, "Prescription and results of physical activity," J Cardiovasc Pharmacol, vol. 25 Suppl 1, pp. S207, 1995.

[38] G. A. Kelley and K. S. Kelley, "Progressive resistance exercise and resting blood pressure : A meta-analysis of randomized controlled trials," Hypertension, vol. 35, no. 3, pp. 838-43, 2000.

[39] K. R. Short, J. L. Vittone, M. L. Bigelow, D. N. Proctor, R. A. Rizza, J. M. Coenen-Schimke, and K. S. Nair, "Impact of aerobic exercise training on age-related changes in insulin sensitivity and muscle oxidative capacity," Diabetes, vol. 52, no. 8, pp. 1888-1896, 2003.

[40] R. J. Sigal, G. P. Kenny, D. H. Wasserman, C. Castaneda-Sceppa, and R. D. White, "Physical activity/exercise and type 2 diabetes a consensus statement from the american diabetes association," Diabetes care, vol. 29, no. 6, pp. 1433-1438, 2006.

[41] S. P. Tokmakidis, C. E. Zois, K. A. Volaklis, K. Kotsa, and A.-M. Touvra, "The effects of a combined strength and aerobic exercise program on glucose control and insulin action in women with type 2 diabetes," European journal of applied physiology, vol. 92, no. 4-5, pp. 437-442, 2004.

[42] H. Yokoyama, M. Emoto, T. Araki, S. Fujiwara, K. Motoyama, T. Morioka, H. Koyama, T. Shoji, Y. Okuno, and Y. Nishizawa, "Effect of aerobic exercise on plasma adiponectin levels and insulin resistance in type 2 diabetes," Diabetes care, vol. 27, no. 7, pp. 1756-1758, 2004.

[43] J. R. Stratton, W. L. Chandler, R. S. Schwartz, M. D. Cerqueira, W. C. Levy, S. E. Kahn, V. G. Larson, K. C. Cain, J. C. Beard, and I. B. Abrass, "Effects of physical conditioning on fibrinolytic variables and fibrinogen in young and old healthy adults," Circulation, vol. 83, no. 5, pp. 1692-7, 1991

[44] A. S. Kestin, P. A. Ellis, M. R. Barnard, A. Errichetti, B. A. Rosner, and A. D. Michelson, "Effect of strenuous exercise on platelet activation state and reactivity," Circulation, vol. 88, no. 4 Pt 1, pp. 1502-11, 1993.

[45] Z. V. Tran and A. Weltman, "Differential effects of exercise on serum lipid and lipoprotein levels seen with changes in body weight. a meta-analysis," JAMA, vol. 254, no. 7, pp. 919-24, 1985.

[46] W. R. Thompson, N. F. Gordon, and L. S. Pescatello, American College of Sports Medicine. Guidelines for Exercise Testing and Prescription. Philadelphia: Lippincott Williams Wilkins, eight ed., 2010.

[47] G. F. Fletcher, G. J. Balady, E. A. Amsterdam, B. Chaitman, R. Eckel, J. Fleg, V. F. Froelicher, A. S. Leon, I. L. Pina, R. Rodney, D. G. Simons-Morton, M. A. Williams, and T. Bazzarre, "Exercise standards for testing and training - a statement for healthcare professionals from the american heart association," Circulation, vol. 104, no. 14, pp. 1694-1740, 2001.

[48] G. A. Borg, “Perceived exertion,” Exerc Sport Sci Rev, vol. 2, pp. 131-153, 1974.

[49] I. F. Charo, L. S. Bekeart, and D. R. Phillips, "Platelet glycoprotein iib-iiia-like proteins mediate endothelial cell attachment to adhesive proteins and the extracellular matrix," J Biol Chem, vol. 262, no. 21, pp. 9935-8, 1987.

[50] M. A. Mittleman, M. Maclure, G. H. Tofler, J. B. Sherwood, R. J. Goldberg, and J. E. Muller, "Triggering of acute myocardial infarction by heavy physical exertion. protection against triggering by regular exertion. determinants of myocardial infarction onset study investigators," $N$ Engl J Med, vol. 329, no. 23, pp. 1677-83, 1993.

[51] S. N. Willich, M. Lewis, H. Lowel, H. R. Arntz, F. Schubert, and R. Schroder, "Physical exertion as a trigger of acute myocardial infarction. triggers and mechanisms of myocardial infarction study group," N Engl J Med, vol. 329, no. 23, pp. 1684-90, 1993.

[52] M. L. Pollock, L. R. Gettman, C. A. Milesis, M. D. Bah, L. Durstine, and R. B. Johnson, "Effects of frequency and duration of training on attrition and incidence of injury," Med Sci Sports, vol. 9, no. 1, pp. 31-6, 1977. 
[53] L. Hermansen and B. Saltin, "Oxygen uptake during maximal treadmill and bicycle exercise," J Appl Physiol, vol. 26, no. 1, pp. 31-7, 1969.

[54] W. D. McArdle, F. I. Katch, and G. S. Pechar, "Comparison of continuous and discontinuous treadmill and bicycle tests for max vo2," Med Sci Sports, vol. 5, no. 3, pp. 156-60, 1973.

[55] G. A. McKay and E. W. Banister, "A comparison of maximum oxygen uptake determination by bicycle ergometry at various pedaling frequencies and by treadmill running at various speeds," Eur J Appl Physiol Occup Physiol, vol. 35, no. 3, pp. 191-200, 1976.

[56] S. N. Koyal, B. J. Whipp, D. Huntsman, G. A. Bray, and K. Wasserman, "Ventilatory responses to the metabolic acidosis of treadmill and cycle ergometry," J Appl Physiol, vol. 40, no. 6, pp. 864-7, 1976.

[57] J. Myers, D. Walsh, N. Buchanan, and V. F. Froelicher, "Can maximal cardiopulmonary capacity be recognized by a plateau in oxygen uptake?," Chest, vol. 96, no. 6, pp. 1312-6, 1989.

[58] J. R. Day, H. B. Rossiter, E. M. Coats, A. Skasick, and B. J. Whipp, "The maximally attainable vo2 during exercise in humans: the peak vs. maximum issue," Journal of applied physiology, vol. 95, no. 5, pp. 19011907, 2003.

[59] H. Loe, O. Rognmo, B. Saltin, and U. Wisloff, "Aerobic capacity reference data in 3816 healthy men and women 20-90 years," PLoS One, vol. 8, no. 5, p. e64319, 2013.

[60] A. M. Bovens, M. A. van Baak, J. G. Vrencken, J. A. Wijnen, W. H. Saris, and F. T. Verstappen, "Maximal aerobic power in cycle ergometry in middle-aged men and women, active in sports, in relation to age and physical activity," Int J Sports Med, vol. 14, no. 2, pp. 66-71, 1993.

[61] J. E. Hansen, R. Casaburi, D. M. Cooper, and K. Wasserman, "Oxygen uptake as related to work rate increment during cycle ergometer exercise,” Eur J Appl Physiol Occup Physiol, vol. 57, no. 2, pp. 140-5, 1988.

[62] R. K. Binder, M. Wonisch, U. Corra, A. Cohen-Solal, L. Vanhees, H. Saner, and J. P. Schmid, "Methodological approach to the first and second lactate threshold in incremental cardiopulmonary exercise testing," Eur $J$ Cardiovasc Prev Rehabil, vol. 15, no. 6, pp. 726-34, 2008.

[63] N. L. Jones, L. Makrides, C. Hitchcock, T. Chypchar, and N. McCartney, "Normal standards for an incremental progressive cycle ergometer test," Am Rev Respir Dis, vol. 131, no. 5, pp. 700-8, 1985.

[64] J. A. Davis, T. W. Storer, and V. J. Caiozzo, "Prediction of normal values for lactate threshold estimated by gas exchange in men and women,” Eur J Appl Physiol Occup Physiol, vol. 76, no. 2, pp. 157-64, 1997.

[65] C. G. Gallagher, E. Brown, and M. Younes, "Breathing pattern during maximal exercise and during submaximal exercise with hypercapnia," J Appl Physiol (1985), vol. 63, no. 1, pp. 238-44, 1987.

[66] B. D. Johnson, K. W. Saupe, and J. A. Dempsey, "Mechanical constraints on exercise hyperpnea in endurance athletes," J Appl Physiol (1985), vol. 73, no. 3, pp. 874-86, 1992.

[67] J. A. Dempsey and M. A. Babcock, "An integrative view of limitations to muscular performance," Adv Exp Med Biol, vol. 384, pp. 393-9, 1995.

[68] J. Stuart, R. J. and M. H. Ellestad, "National survey of exercise stress testing facilities," Chest, vol. 77, no. 1, pp. 94-7, 1980 .

[69] K. Strong, C. Mathers, and R. Bonita, "Preventing stroke: saving lives around the world," Lancet Neurol, vol. 6, no. 2, pp. 182-7, 2007.

[70] "Atlas of heart disease and stroke," World Health Organization, 2004. 
[71] E. C. Jauch, B. Cucchiara, O. Adeoye, W. Meurer, J. Brice, Y. Chan, N. Gentile, and M. F. Hazinski, "Part 11: Adult stroke 2010 american heart association guidelines for cardiopulmonary resuscitation and emergency cardiovascular care," Circulation, vol. 122, no. 18, pp. S818-S828, 2010.

[72] P. W. Duncan, R. Zorowitz, B. Bates, J. Y. Choi, J. J. Glasberg, G. D. Graham, R. C. Katz, K. Lamberty, and D. Reker, "Management of adult stroke rehabilitation care - a clinical practice guideline," Stroke, vol. 36, no. 9, pp. E100-E143, 2005.

[73] H. S. Jorgensen, H. Nakayama, H. O. Raaschou, J. Vive-Larsen, M. Stoier, and T. S. Olsen, "Outcome and time course of recovery in stroke. part i: Outcome. the copenhagen stroke study," Arch Phys Med Rehabil, vol. 76 , no. 5, pp. 399-405, 1995.

[74] H. S. Jorgensen, H. Nakayama, H. O. Raaschou, and T. S. Olsen, "Recovery of walking function in stroke patients: the copenhagen stroke study," Arch Phys Med Rehabil, vol. 76, no. 1, pp. 27-32, 1995.

[75] R. C. Lo, "Recovery and rehabilitation after stroke," Can Fam Physician, vol. 32, pp. 1851-3, 1986.

[76] J. Astrup, B. K. Siesjo, and L. Symon, "Thresholds in cerebral ischemia - the ischemic penumbra," Stroke, vol. 12 , no. 6 , pp. $723-5,1981$.

[77] Y. Inoue, K. Takemoto, T. Miyamoto, N. Yoshikawa, S. Taniguchi, S. Saiwai, Y. Nishimura, and T. Komatsu, "Sequential computed tomography scans in acute cerebral infarction," Radiology, vol. 135, no. 3, pp. 655-62, 1980 .

[78] R. J. Nudo, "Adaptive plasticity in motor cortex: implications for rehabilitation after brain injury," J Rehabil Med, no. 41 Suppl, pp. 7-10, 2003

[79] P. M. Rossini, C. Calautti, F. Pauri, and J. C. Baron, "Post-stroke plastic reorganisation in the adult brain," Lancet Neurol, vol. 2, no. 8, pp. 493-502, 2003.

[80] M. Hallett, "Plasticity of the human motor cortex and recovery from stroke," Brain Res Brain Res Rev, vol. 36, no. 2-3, pp. 169-74, 2001.

[81] H. S. Jorgensen, H. Nakayama, H. O. Raaschou, J. Vivelarsen, M. Stoier, and T. S. Olsen, "Outcome and time course of recovery in stroke. part ii: Time course of recovery. the copenhagen stroke study," Archives of Physical Medicine and Rehabilitation, vol. 76, no. 5, pp. 406-412, 1995.

[82] P. W. Duncan, L. B. Goldstein, D. Matchar, G. W. Divine, and J. Feussner, "Measurement of motor recovery after stroke. outcome assessment and sample size requirements,” Stroke, vol. 23, no. 8, pp. 1084-9, 1992.

[83] L. Ferrucci, S. Bandinelli, J. M. Guralnik, M. Lamponi, C. Bertini, M. Falchini, and A. Baroni, "Recovery of functional status after stroke. a postrehabilitation follow-up study," Stroke, vol. 24, no. 2, pp. 200-5, 1993.

[84] P. Langhorne, R. Wagenaar, and C. Partridge, "Physiotherapy after stroke: more is better?," Physiother Res Int, vol. 1, no. 2, pp. 75-88, 1996.

[85] G. Kwakkel, R. C. Wagenaar, T. W. Koelman, G. J. Lankhorst, and J. C. Koetsier, "Effects of intensity of rehabilitation after stroke. a research synthesis," Stroke, vol. 28, no. 8, pp. 1550-6, 1997.

[86] G. Kwakkel, R. van Peppen, R. C. Wagenaar, S. Wood Dauphinee, C. Richards, A. Ashburn, K. Miller, N. Lincoln, C. Partridge, I. Wellwood, and P. Langhorne, "Effects of augmented exercise therapy time after stroke: a meta-analysis," Stroke, vol. 35, no. 11, pp. 2529-39, 2004.

[87] D. X. Cifu and D. G. Stewart, "Factors affecting functional outcome after stroke: a critical review of rehabilitation interventions," Arch Phys Med Rehabil, vol. 80, no. 5 Suppl 1, pp. S35-9, 1999.

[88] C. C. Chen, A. W. Heinemann, C. V. Granger, and R. T. Linn, "Functional gains and therapy intensity during subacute rehabilitation: a study of 20 facilities," Arch Phys Med Rehabil, vol. 83, no. 11, pp. 1514-23, 2002. 
[89] M. J. MacKay-Lyons and L. Makrides, "Longitudinal changes in exercise capacity after stroke," Archives of Physical Medicine and Rehabilitation, vol. 85, no. 10, pp. 1608-1612, 2004.

[90] M. P. Kilgard and M. M. Merzenich, "Cortical map reorganization enabled by nucleus basalis activity," Science, vol. 279, no. 5357, pp. 1714-1718, 1998.

[91] B. French, L. H. Thomas, M. J. Leathley, C. J. Sutton, J. McAdam, A. Forster, P. Langhorne, C. I. M. Price, A. Walker, and C. L. Watkins, "Repetitive task training for improving functional ability after stroke," Cochrane Database of Systematic Reviews, no. 4, p. CD006073, 2007.

[92] N. A. Bayona, J. Bitensky, K. Salter, and R. Teasell, "The role of task-specific training in rehabilitation therapies," Top Stroke Rehabil, vol. 12, no. 3, pp. 58-65, 2005.

[93] E. J. Roth, "Heart disease in patients with stroke: incidence, impact, and implications for rehabilitation. part 1: Classification and prevalence," Archives of Physical Medicine and Rehabilitation, vol. 74, no. 7, pp. 752-760, 1993.

[94] E. J. Roth, "Heart disease in patients with stroke. part 2: Impact and implications for rehabilitation," Archives of Physical Medicine and Rehabilitation, vol. 75, no. 1, pp. 94-101, 1994.

[95] G. E. Gresham, T. F. Phillips, P. A. Wolf, P. M. McNamara, W. B. Kannel, and T. R. Dawber, "Epidemiologic profile of long-term stroke disability: the framingham study," Archives of Physical Medicine and Rehabilitation, vol. 60 , no. 11 , pp. 487-491, 1979.

[96] P. A. Wolf, G. P. Clagett, J. D. Easton, L. B. Goldstein, P. B. Gorelick, M. Kelly-Hayes, R. L. Sacco, and J. P. Whisnant, "Preventing ischemic stroke in patients with prior stroke and transient ischemic attack : a statement for healthcare professionals from the stroke council of the american heart association," Stroke, vol. 30, no. 9, pp. 1991-4, 1999.

[97] A. Tang, K. M. Sibley, S. G. Thomas, W. E. McIlroy, and D. Brooks, "Maximal exercise test results in subacute stroke," Archives of Physical Medicine and Rehabilitation, vol. 87, no. 8, pp. 1100-1105, 2006.

[98] J. O. Kelly, S. L. Kilbreath, G. M. Davis, B. Zeman, and J. Raymond, "Cardiorespiratory fitness and walking ability in subacute stroke patients," Archives of Physical Medicine Rehabilitation, vol. 84, no. 12, pp. 1780$1785,2003$.

[99] D. Brooks, A. Tang, K. M. Sibley, and W. E. McIlroy, "Profile of patients at admission into an inpatient stroke rehabilitation programme: cardiorespiratory fitness and functional characteristics," Physiotherapy Canada vol. 60 , no. 2 , pp. 171-179, 2008.

[100] J. J. Eng, A. S. Dawson, and K. S. Chu, "Submaximal exercise in persons with stroke: Test-retest reliability and concurrent validity with maximal oxygen consumption," Archives of Physical Medicine and Rehabilitation, vol. 85 , no. 1 , pp. $113-118,2004$.

[101] J. Ramas, A. Courbon, F. Roche, F. Bethoux, and P. Calmels, "Effect of training programs and exercise in adult stroke patients: literature review," Annales de Readaptation et de Medecine Physique, vol. 50, no. 6, pp. 438-444, 2007.

[102] F. M. Ivey, R. F. Macko, A. S. Ryan, and C. E. Hafer-Macko, “Cardiovascular health and fitness after stroke,' Topics in Stroke Rehabilitation, vol. 12, no. 1, pp. 1-16, 2005.

[103] M. J. MacKay-Lyons and L. Makrides, "Exercise capacity early after stroke," Archives of Physical Medicine and Rehabilitation, vol. 83, no. 12, pp. 1697-1702, 2002.

[104] N. F. Gordon, M. Gulanick, F. Costa, G. Fletcher, B. A. Franklin, E. J. Roth, and T. Shephard, "Physical activity and exercise recommendations for stroke survivors - an american heart association scientific statement from the council on clinical cardiology, subcommittee on exercise, cardiac rehabilitation, and prevention; the council on cardiovascular nursing; the council on nutrition, physical activity, and metabolism; and the stroke council," Stroke, vol. 35, no. 5, pp. 1230-1240, 2004. 
[105] E. J. Roth, K. Mueller, and D. Green, "Stroke rehabilitation outcome - impact of coronary-artery disease," Stroke, vol. 19, no. 1, pp. 42-47, 1988.

[106] V. Convertino, J. Hung, D. Goldwater, and R. F. DeBusk, "Cardiovascular responses to exercise in middle-aged men after 10 days of bedrest," Circulation, vol. 65, no. 1, pp. 134-40, 1982.

[107] B. A. Franklin and W. Sanders, "Reducing the risk of heart disease and stroke," Phys Sportsmed, vol. 28, no. 10, pp. 19-26, 2000.

[108] M. Y. C. Pang, J. J. Eng, A. S. Dawson, and S. Gylfadottir, "The use of aerobic exercise training in improving aerobic capacity in individuals with stroke: a meta-analysis," Clinical Rehabilitation, vol. 20, no. 2, pp. 97-111, 2006.

[109] P. S. Lum, C. G. Burgar, and P. C. Shor, "Evidence for improved muscle activation patterns after retraining of reaching movements with the mime robotic system in subjects with post-stroke hemiparesis," Neural Systems and Rehabilitation Engineering, IEEE Transactions on, vol. 12, no. 2, pp. 186-194, 2004.

[110] M. L. Aisen, H. I. Krebs, N. Hogan, F. McDowell, and B. T. Volpe, "The effect of robot-assisted therapy and rehabilitative training on motor recovery following stroke," Archives of Neurology, vol. 54, no. 4, p. 443, 1997.

[111] S. Hesse, G. Schulte-Tigges, M. Konrad, A. Bardeleben, and C. Werner, "Robot-assisted arm trainer for the passive and active practice of bilateral forearm and wrist movements in hemiparetic subjects," Archives of physical medicine and rehabilitation, vol. 84, no. 6, pp. 915-920, 2003.

[112] S. Masiero, A. Celia, M. Armani, and G. Rosati, "A novel robot device in rehabilitation of post-stroke hemiplegic upper limbs," Aging clinical and experimental research, vol. 18, no. 6, pp. 531-535, 2006.

[113] T. Nef, M. Mihelj, G. Colombo, and R. Riener, "Armin-robot for rehabilitation of the upper extremities," pp. 3152-3157, IEEE, 2006.

[114] D. J. Reinkensmeyer, L. E. Kahn, M. Averbuch, A. McKenna-Cole, B. D. Schmit, and W. Z. Rymer, "Understanding and treating arm movement impairment after chronic brain injury: progress with the arm guide," Journal of rehabilitation research and development, vol. 37, no. 6, pp. 653-662, 2000.

[115] G. Fazekas, M. Horvath, T. Troznai, and A. Toth, "Robot-mediated upper limb physiotherapy for patients with spastic hemiparesis: A preliminary study," Journal of Rehabilitation Medicine, vol. 39, no. 7, pp. 580-582, 2007.

[116] A. Mayr, M. Kofler, and L. Saltuari, "Armor: An electromechanical robot for upper limb training following stroke. a prospective randomised controlled pilot study," Handchirurgie, Mikrochirurgie, plastische Chirurgie, vol. 40, no. 1, pp. 66-73, 2008.

[117] R. J. Sanchez, J. Liu, S. Rao, P. Shah, R. Smith, T. Rahman, S. C. Cramer, J. E. Bobrow, and D. J. Reinkensmeyer, "Automating arm movement training following severe stroke: functional exercises with quantitative feedback in a gravity-reduced environment," Neural Systems and Rehabilitation Engineering, IEEE Transactions on, vol. 14, no. 3, pp. 378-389, 2006.

[118] G. Colombo, M. Joerg, R. Schreier, and V. Dietz, "Treadmill training of paraplegic patients using a robotic orthosis," Journal of Rehabilitation Research and Development, vol. 37, no. 6, pp. 693-700, 2000.

[119] S. Freivogel, J. Mehrholz, T. Husak-Sotomayor, and D. Schmalohr, "Gait training with the newly developed'lokohelp'-system is feasible for non-ambulatory patients after stroke, spinal cord and brain injury. a feasibility study," Brain Injury, vol. 22, no. 7-8, pp. 625-632, 2008.

[120] D. Reinkensmeyer, J. H. Wynne, and S. J. Harkema, "A robotic tool for studying locomotor adaptation and rehabilitation," vol. 3, pp. 2353-2354, IEEE, 2002. 
[121] D. J. Reinkensmeyer, D. Aoyagi, J. L. Emken, J. A. Galvez, W. Ichinose, G. Kerdanyan, S. Maneekobkunwong, K. Minakata, J. A. Nessler, and R. Weber, "Tools for understanding and optimizing robotic gait training," Journal of rehabilitation research and development, vol. 43, no. 5, p. 657, 2006.

[122] S. K. Banala, S. K. Agrawal, and J. P. Scholz, "Active leg exoskeleton (alex) for gait rehabilitation of motorimpaired patients," in IEEE 10th International Conference on Rehabilitation Robotics, pp. 401-407, IEEE, 2007.

[123] J. F. Veneman, R. Kruidhof, E. E. G. Hekman, R. Ekkelenkamp, E. H. F. Van Asseldonk, and H. van der Kooij, "Design and evaluation of the lopes exoskeleton robot for interactive gait rehabilitation," Neural Systems and Rehabilitation Engineering, IEEE Transactions on, vol. 15, no. 3, pp. 379-386, 2007.

[124] S. Hesse, A. Waldner, and C. Tomelleri, "Innovative gait robot for the repetitive practice of floor walking and stair climbing up and down in stroke patients," J Neuroeng Rehabil, vol. 7, p. 30, 2010.

[125] C. Schmitt, P. Métrailler, A. Al-Khodairy, R. Brodard, J. Fournier, M. Bouri, and R. Clavel, "The motion maker ${ }^{\mathrm{TM}}$ : a rehabilitation system combining an orthosis with closed-loop electrical muscle stimulation," pp. 117-20, 2004.

[126] J. Patton, D. A. Brown, M. Peshkin, J. J. Santos-Munné, A. Makhlin, E. Lewis, E. J. Colgate, and D. Schwandt, "Kineassist: design and development of a robotic overground gait and balance therapy device," Topics in stroke rehabilitation, vol. 15, no. 2, pp. 131-139, 2008.

[127] M. Bouri, Y. Stauffer, C. Schmitt, Y. Allemand, S. Gnemmi, R. Clavel, P. Metrailler, and R. Brodard, "The walktrainer: a robotic system for walking rehabilitation," pp. 1616-1621, IEEE, 2006.

[128] A. Goffer, "Gait-locomotor apparatus," Patent: 7153 242, 2006.

[129] H. Kawamoto and Y. Sankai, Power assist system HAL-3 for gait disorder person. Computers helping people with special needs, Springer, 2002.

[130] J. Mehrholz, A. Hadrich, T. Platz, J. Kugler, and M. Pohl, "Electromechanical and robot-assisted arm training for improving generic activities of daily living, arm function, and arm muscle strength after stroke," Cochrane Database Syst Rev, vol. 6, p. CD006876, 2012.

[131] J. Mehrholz, B. Elsner, C. Werner, J. Kugler, and M. Pohl, "Electromechanical-assisted training for walking after stroke," Cochrane Database Syst Rev, vol. 7, p. CD006185, 2013.

[132] C. Krewer, F. Müller, B. Husemann, S. Heller, J. Quintern, and E. Koenig, "The influence of different lokomat walking conditions on the energy expenditure of hemiparetic patients and healthy subjects," Gait Posture, vol. 26, no. 3, pp. 372-377, 2007.

[133] M. S. Nash, P. L. Jacobs, B. M. Johnson, and E. Field-Fote, "Metabolic and cardiac responses to roboticassisted locomotion in motor-complete tetraplegia: A case report," Journal of Spinal Cord Medicine, vol. 27, no. 1, pp. 78-82, 2004.

[134] M. Turiel, S. Sitia, S. Cicala, V. Magagnin, I. Bo, A. Porta, E. Caiani, C. Ricci, V. Licari, V. D. G. Colonna, and L. Tomasoni, "Robotic treadmill training improves cardiovascular function in spinal cord injury patients," International Journal of Cardiology, vol. 149, no. 3, pp. 323-329, 2011.

[135] K. J. Hunt, L. P. Jack, A. Pennycott, C. Perret, M. Baumberger, and T. H. Kakebeeke, "Control of work ratedriven exercise facilitates cardiopulmonary training and assessment during robot-assisted gait in incomplete spinal cord injury," Biomedical Signal Processing and Control, vol. 3, no. 1, pp. 19-28, 2008.

[136] A. Pennycott, K. J. Hunt, L. P. Jack, C. Perret, and T. H. Kakebeeke, "Estimation and volitional feedback control of active work rate during robot-assisted gait," Control Engineering Practice, vol. 17, no. 2, pp. 322-328, 2008. 



\section{Chapter 2}

\section{Effects of cardiovascular exercise early after stroke: systematic review and meta-analysis}

BMC Neurology 2012;12:45

doi: 10.1186/1471-2377-12-45

Oliver Stoller Eling D de Bruin Ruud H Knols

Kenneth J Hunt 


\section{Abstract}

Background: Previous studies have shown the beneficial effects of aerobic exercise in chronic stroke. Most motor and functional recovery occurs in the first months after stroke. Improving cardiovascular capacity may have potential to precipitate recovery during early stroke rehabilitation. Currently, little is known about the effects of early cardiovascular exercise in stroke survivors. The aim of this systematic review was to evaluate the effectiveness of cardiovascular exercise early after stroke.

Methods: A systematic literature search was performed. For this review, randomised and nonrandomised prospective controlled cohort studies using a cardiovascular, cardiopulmonary or aerobic training intervention starting within 6 months post-stroke were considered. The PEDro scale was used to detect risk of bias in individual studies. Inter-rater agreement was calculated (kappa). Meta-analysis was performed using a random-effects model.

Results: A total of 11 trials were identified for inclusion. Inter-rater agreement was considered to be "very good" (Kappa: 0.81, Standard Error: 0.06, CI95\%: 0.70-0.92), and the methodological quality was "good" (7 studies) to "fair" (4 studies). Peak oxygen uptake data were available for 155 participants. Pooled analysis yielded homogenous effects favouring the intervention group ( standardised mean difference $(\mathrm{SMD})=0.83, \mathrm{CI} 95 \%=0.50-1.16, \mathrm{Z}=4.93, \mathrm{p}<0.01)$. Walking endurance assessed with the 6 Minute Walk Test comprised 278 participants. Pooled analysis revealed homogenous effects favouring the cardiovascular training intervention group (SMD = 0.69$, CI95\% $=0.45-0.94, Z=5.58, \mathrm{p}<0.01)$. Gait speed, measured in 243 participants, did not show significant results $(\mathrm{SMD}=0.51, \mathrm{CI} 95 \%=-0.25-1.26, \mathrm{Z}=1.31, \mathrm{p}=0.19)$ in favour of early cardiovascular exercise.

Conclusion: This meta-analysis shows that stroke survivors may benefit from cardiovascular exercise during subacute stages to improve peak oxygen uptake and walking distance. Thus, cardiovascular exercise should be considered in subacute stroke rehabilitation. However, concepts to influence and evaluate aerobic capacity in severely affected individuals with subacute stroke, as well as in the very early period after stroke, are lacking. Further research is needed to develop appropriate methods for cardiovascular rehabilitation early after stroke and to evaluate long-term effects of cardiovascular exercise on aerobic capacity, physical functioning, and quality of life. 


\section{Background}

Each year 15 million people worldwide experience a stroke. About one third of this population will remain disabled [1]. According to European population projections from the United Nations the number of new cases of stroke will increase from 1.1 million to 1.5 million in 2025 [2]. Furthermore, it was estimated that stroke will occur in $35 \%$ of the population over the age of 65 , a group that will increase in proportion due to demographic shifts in most populations [3]. This will presumably lead to an increased need for rehabilitation programs to enhance recovery, improve functional status and quality of life, thus leading to future challenges for societies, and healthcare and financial systems. Rehabilitation strategies for stroke survivors have focused primarily on restoring reduced motor control of the affected side as well as postural control [4, 5]. However, less than a third of the variance in disability after stroke can be explained by the extent of neurological impairment [6]. Around $75 \%$ of post-stroke individuals exhibit cardiac disease [7, 8]. Thus stroke survivors may be more disabled by associated cardiac disease than by the stroke incident itself [9].

Individuals after stroke generally have low endurance for exercise as a secondary consequence of immobility $[10,11]$. Previous work has shown that maximum oxygen uptake $\left(\mathrm{V}_{2} \max \right)$ is reduced to $10-17 \mathrm{~mL} / \mathrm{min} / \mathrm{kg}$ within 0-30 days after the stroke [12, 13, 14], and does not rise above $20 \mathrm{~mL} / \mathrm{min} / \mathrm{kg}$ after 6 months $[11,14,15,16]$. These values are $25-45 \%$ lower than $\dot{\mathrm{VO}_{2}} \max$ in age-matched, healthy subjects $[17,18]$. This early and persistent decline in aerobic capacity can delay or inhibit participation in a therapeutic exercise program, complicate the rehabilitation process and long-term post-stroke course of care, and limit the ability of the individual to perform functional activities independently $[8,19]$. Previous work has shown that subjects with chronic stroke will reach their maximum aerobic capacity during activities of daily living [18]. Therefore, a minor increase in aerobic capacity could mean the difference between dependency and independence during all daily living.

From these observations it can be concluded that cardiovascular training early after stroke may be a useful treatment option. Several controlled clinical trials evaluated the effect of cardiovascular exercise in individuals post-stroke [11, 20, 21, 22, 23, 24, 25, 26]. The results of these studies are summarised in a meta-analysis demonstrating significant homogenous standard effects sizes for improving aerobic capacity, walking speed, and walking endurance in individuals with stroke. However, the main focus of the studies included was on the chronic stage after stroke $(\geq 6$ months after the initial event) [27]. Whether these findings can be generalised, and whether such training therefore is also feasible for a population in the acute and subacute stages following stroke, remains unclear. 
Until now, little is known about the effects of cardiovascular training in the early stages of stroke. Given the fact that most motor and functional recovery occurs in the first months after stroke [28], there could be a hitherto neglected potential to precipitate motor recovery by improving cardiovascular capacity in individuals early after stroke. A physical exercise program with an adequate emphasis on cardiovascular parameters might be hypothesised to induce a training effect, which could facilitate improved physical functioning and lead to enhanced quality of life.

The purpose of this systematic review is to provide an overview of the currently available evidence for the use of cardiovascular training in the early stages after stroke. The aim is to identify strategies that have the potential to affect physical functioning and that might be used in future early intervention type studies for individuals with stroke. The following specific questions were evaluated: (1) What is the level of evidence for cardiovascular exercise interventions to influence aerobic capacity and physical functioning implemented within 6 months after the initial stroke event?; (2) How soon after the initial stroke event is cardiovascular exercise introduced?; (3) What is the common practice for measurement of aerobic capacity early after stroke?

\section{Methods}

\section{Protocol}

Analysis methods and inclusion criteria were specified in advance and documented in the review protocol, which can be found in Additional file 1 (see online publication).

\section{Data sources and search strategy}

We searched the databases MEDLINE/Premedline (OvidSP), EMBASE, Cochrane Library, CINAHL, and ISI Web of Science (WOS), and performed an additional focussed search where stroke had to be in the title or in the subject headings. The final search was done on 24 November 2011. We used a combination of medical subject headings $(\mathrm{MeSH})$ and textwords as search terms, including the following main terms for the population: Stroke, Cerebral Stroke, Vascular Accident, Brain Vascular Accident, Apoplexy, Cerebrovascular Apoplexy, Cerebrovascular Stroke, CVA (Cerebrovascular Accident), Cerebrovascular Accident, Acute Stroke, Acute Cerebrovascular Accident, Acute $=0-6$ months post-stroke, age $\geq 18$ years. For the intervention of interest: cardiovascular training, cardiopulmonary training, cardiorespiratory training, aerobic training, endurance training, exercise, endurance exercise, ergometry, cycling, rowing, treadmill. For the outcomes of interest: cardiovascular fitness, aerobic fitness, condition, endurance, physical conditioning, $\dot{\mathrm{V}} \mathrm{O}_{2}$ maximal, 
$\dot{\mathrm{V}} \mathrm{O}_{2}$ maximum, $\dot{\mathrm{VO}}_{2}$ peak, maximal oxygen uptake, heart rate, neural recovery, neural rehabilitation, functional recovery, function recovery, quality of life. Articles found through hand search by scanning reference lists of identified studies supplemented the search results.

\section{Eligibility criteria}

We included randomised and non-randomised prospective controlled cohort studies considering cardiovascular training in the subacute stages after stroke. No language or publication date restrictions were imposed and only peer-reviewed journal articles were included. Participants (age $\geq 18$ years) with initial stroke in the acute phase deemed medically stable enough to participate in an aerobic exercise intervention were considered. For the purpose of this systematic review, the phase after stroke was defined by the start of the intervention, whereas we defined acute as the first week after the stroke event, and subacute as 7 days - 6 month after stroke onset. All types of stroke and all severity levels were included. Furthermore, we considered only studies using cardiovascular, cardiopulmonary or aerobic training interventions. Typically, cardiovascular training is performed for extended periods of time on devices that allow for recruitment of large muscle groups or on ergometers (e.g. treadmill, cycling, rowing), or by utilising modes of activity such as walking, cycling, rowing or stair climbing. Any outcome representing an objective and/or subjective measure in the field of cardiovascular fitness, physical condition, endurance, oxygen uptake, heart rate, neural recovery, functional recovery, quality of life, etc. was included in this review. The primary outcome measures were directly related to physical functioning and/or physical performance using validated parameters or scales.

\section{Study selection}

After duplicate citations were removed, two reviewers (OS, EDB) performed the identification and the eligibility assessment independently in a blinded standardised manner by scanning the titles, abstracts and keywords. Disagreements between reviewers were resolved by consensus. If no consensus was found, a third reviewer $(\mathrm{KH})$ made the final decision.

\section{Data collection process}

We developed a data extraction sheet based on the Cochrane Handbook for Systematic Reviews of Interventions data extraction template (The Cochrane Collaboration, Oxford, England). One reviewer (OS) extracted the data from included studies and a second reviewer (EDB) crosschecked the extracted data for accuracy. Disagreements were resolved by discussion between two reviewers 
(OS, EDB); if no agreement could be reached, a third author $(\mathrm{KH})$ verified the data and made the final decision. We contacted authors by email or telephone for further information about unpublished and unclear data. We extracted the following information: study ID (author and year), title, design, intervention length, setting, number of participants, participants missing, inclusion criteria, exclusion criteria, age, sex, country, stroke type, location, severity, initial diagnostics, time since stroke, number of participants, experimental and control interventions, and additional outcome measures.

\section{Risk of bias in individual studies}

Two independent reviewers (OS, EDB) assessed all studies for risk of bias. The PEDro scale was used for this assessment. The PEDro scale was developed to assess the methodological quality of physical therapy RCTs and is composed of 11 items rating internal validity (10 items) and external validity ( 1 item) of a clinical trial. The last item does not influence the internal or statistical validity of the trial and, in general, is not used to calculate the PEDro scale (partitioned) score. The reliability of the PEDro scale has been demonstrated [29]. Consistent with PEDro, we only considered the internal validity (10 items) to assess risk of bias, and used the following cut-points: 9-10 (excellent); 6-8 (good); 4-5 (fair); <4 (poor). Disagreements during the quality assessments were resolved by discussion between two review authors (OS, EDB); if no agreement could be reached, a third author $(\mathrm{KH})$ verified the data and made the final decision.

\section{Risk of bias across studies}

We assessed the possibility of publication bias by evaluating funnel plots of the trials mean differences for asymmetry, which can result from non-publication of small trials with negative results. Heterogeneity of effect sizes was evaluated by $\mathrm{I}^{2}$ statistics, where at least $50 \%$ was taken as an indicator of substantial heterogeneity.

\section{Analysis}

Review Manager (The Cochrane Collaboration, Oxford, England) and Microsoft Excel (2011) were used to calculate mean differences (MD), standard deviation (SD), effects sizes (ES), confidence intervals (CI), and p-values (p). In a few instances, missing SDs of MDs and standard errors (SE) were calculated according to the Cochrane Handbook for Systematic Reviews (The Cochrane Collaboration, Oxford, England). For outcomes where sufficient data was available, we performed 
the meta-analyses by computing standardised mean differences (SMD) using random-effects models. Percentage agreement and Cohens kappa were calculated and interpreted in accordance with Landis and Kochs benchmarks for assessing the agreement between raters: poor $(\leq 0)$, slight $(0.0$ $0.20)$, fair (0.21-0.40), moderate (0.41-0.60), substantial (0.61-0.80), and almost perfect (0.81-1.0) [30]. The PRISMA-statement was followed for reporting items of this systematic review [31].

\section{Results}

\section{Study selection}

The search of MEDLINE/Premedline (OvidSP), EMBASE, Cochrane Library, CINHAL, and ISI Web of Science (WOS) provided a total of 803 citations, and 492 citations with stroke in the title and/or keywords. 538 remained after adjusting for duplicates. 334 studies were discarded after reviewing the abstracts, because they clearly did not meet the inclusion criteria. 174 studies were excluded because subjects were not in the subacute phase after stroke at baseline of the interventions. The full text of the remaining 30 citations was examined in more detail. 19 of these studies did not meet the inclusion criteria. Finally, 11 studies fulfilled all criteria and were selected for review [32, 33, 34, 35, 36, 37, 38, 39, 40, 41, 42] (Figure 2.1).

\section{Study characteristics}

The study characteristics are shown in Table 2.1. Ten studies were randomised controlled trials or randomised controlled pilot studies, and 1 study was a prospective controlled matched design [41]. All articles were published in peer-reviewed journals in English. The year of publication ranged from 1998 to 2011. All studies were carried out during inpatient rehabilitation and were from the United States (3), Germany (2), Israel (4), France (1), and Canada (1). The outcomes of the 3 studies from Katz-Leurer et al. [36, 37, 38] are based on the same sample, but used different outcome measures.

The studies included involved 423 participants with mild to moderate deficits in motor function and functional abilities. The main inclusion criteria for the participants in the studies were: stroke in the acute and subacute phase (0-6 month after initial event), ability to ambulate minimally (measured using Functional Ambulation Classification (FAC), Orpington Prognostic Scale, FuglMeyer Score, Barthel-Index, 10 Meter Walk Test, Chedoke-McMaster Stroke Assessment), and appropriate cognitive function (measured using Mini Mental State Examination). The main exclusion criterion was serious cardiac contraindications for exercise testing according to the ACSM [43]. 


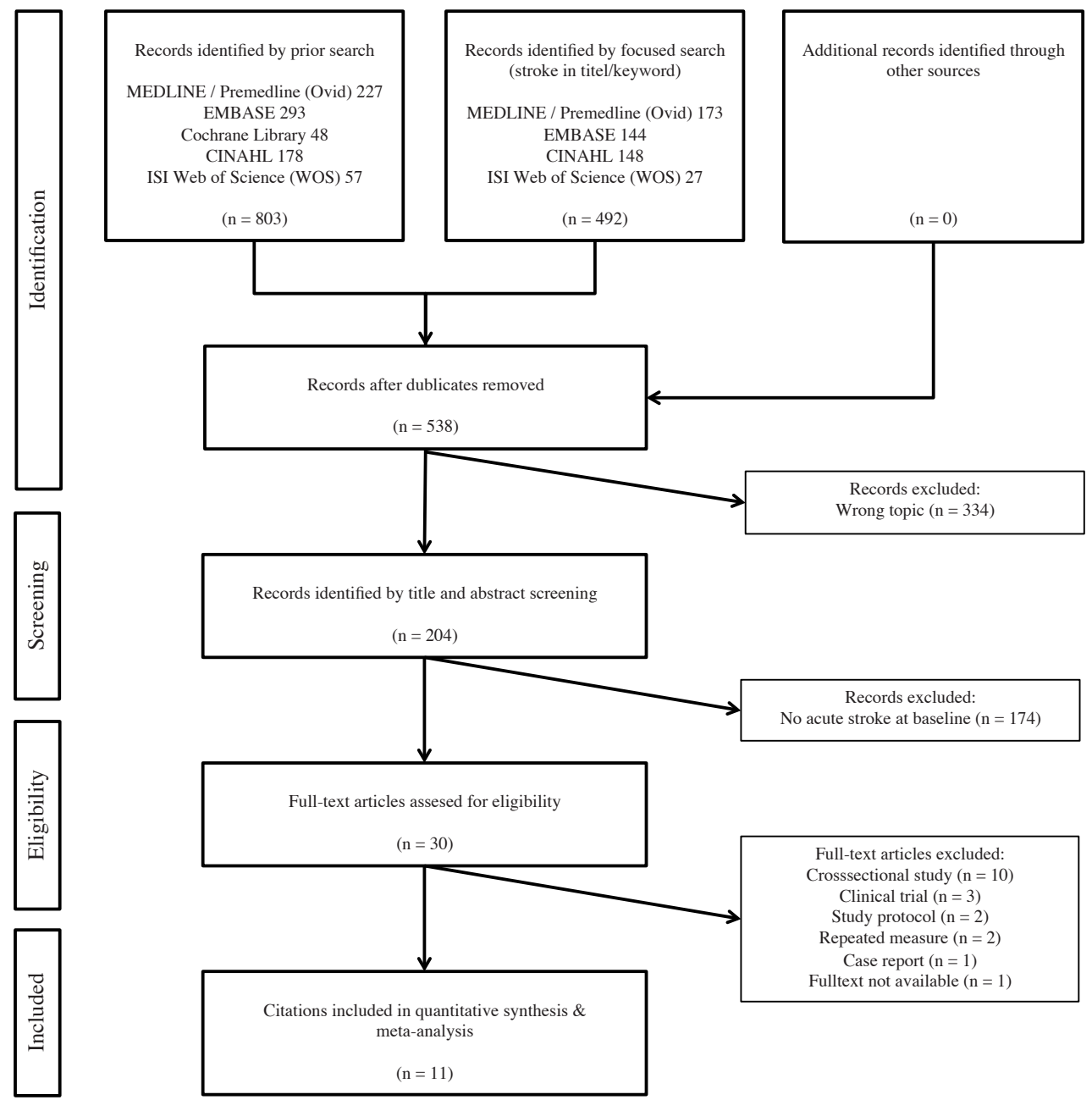

Figure 2.1: Results of the systematic review. Studies flow chart for the systematic review and meta-analysis.

Leg cycle ergometry (5 studies) and treadmill training (4 studies) were the most common methods for aerobic exercise in the subacute phase following stroke. One study used task-orientated circuit class training [40]. Letombe et al. [39] and Toledano-Zarhi et al. [42] mixed the interventions using leg cycle ergometry or treadmill training or aerobic exercise with a stepper or hand bike $[39,42]$. Training duration ranged from 3 to 13 weeks (mean $6.56 \pm 3.7$ weeks), whereas training intensity ranged from $40-80 \%$ of heart rate reserve. All reviewed studies used an intervention pro- 
tocol consisting of continuous exercise of 20-90 min (mean $47.78 \pm 25.8 \mathrm{~min}$ ) for 25 sessions per week (mean $3.44 \pm 1.0$ sessions/week). The intervention started $34.28 \pm 25.1$ days after the initial stroke event. The earliest time was 6 days post-stroke [41, 42].

Outcomes which were directly related to aerobic capacity included peak oxygen uptake ( $\dot{\mathrm{VO}}_{2}$ peak), peak work rate (Ppeak), peak heart rate (HRpeak), heart rate variability, or the 6 Minute Walk Test (6MWT). Usually, $\dot{\mathrm{V}} \mathrm{O}_{2}$ peak was evaluated using semi-recumbent leg cycle ergo-spirometry. Only da Cunha et al. [32] measured oxygen uptake during a 5-minute walk using a portable gas analyser (KB1-C system). The 6MWT was used in 7 of 11 studies, whereas da Cunha et al. used a 5-minute walk test [32]. HRpeak was only evaluated by Tang et al. [41].

Further outcomes were gait speed, motor recovery and functional ability (Rivermead Motor Assessment Score, Functional Ambulation Classification (FAC), Fugl-Meyer Scale, Barthel-Index, Lawton Instrumental ADL, Functional Independence Measure (FIM), Frenchay Activities Index (FAI)), Balance (Berg Balance Scale, Functional Reach Test), hand function (Wolf Motor Function Test, Jebsen Test, grip strength), stair ascent and descent, and quality of life using the Stroke Impact Scale (SIS).

\section{Risk of bias within studies}

The methodological quality of the studies included is summarised in Table 2.2. The most common quality problems were absence of blinding of the assessors (8 studies), absence of concealment of allocation (7 studies), and absence of an intention-to-treat analysis (4 studies). The inter-rater agreement of the quality assessment was considered to be very good (Kappa: 0.81, SE: 0.06, CI95\%: 0.70-0.92).

\section{Syntheses of results}

The synthesis includes only outcomes $\dot{\mathrm{VO}}{ }_{2}$ peak, 6MWT distance and gait speed, where there was sufficient evidence to pool the data. $\dot{\mathrm{VO}} 2$ peak $(\mathrm{mL} / \mathrm{min} / \mathrm{kg})$ data were available in 3 studies, which randomised a total of 173 participants and reported data from 155 participants [34, 39, 41]. Pooled analysis for $\dot{\mathrm{VO}_{2}}$ peak yielded homogenous effects favouring the intervention group ( $\mathrm{SMD}=0.83$, $\mathrm{CI} 95 \%=0.50-1.16, \mathrm{Z}=4.93, \mathrm{p}<0.01$ ) (Figure 2.2). The 6MWT $(\mathrm{m})$ was assessed in 6 studies involving 278 participants, while the baseline sample comprised 298 participants [33, 34, 35, 40, $41,42]$. Pooled analysis for the results of the 6MWT revealed homogenous effects favouring the cardiovascular training intervention group $(\mathrm{SMD}=0.69, \mathrm{CI} 95 \%=0.45-0.94, \mathrm{Z}=5.58, \mathrm{p}<0.01)$ (Figure 2.3). 
Gait speed $(\mathrm{m} / \mathrm{s})$ measured in 5 studies involving 243 participants did not reveal significant effects attributable to a cardiovascular intervention $(\mathrm{SMD}=0.51, \mathrm{CI} 95 \%=-0.25-1.26, \mathrm{Z}=1.31, \mathrm{p}=0.19)$ $[32,34,35,40,41]$ (Figure 2.4).

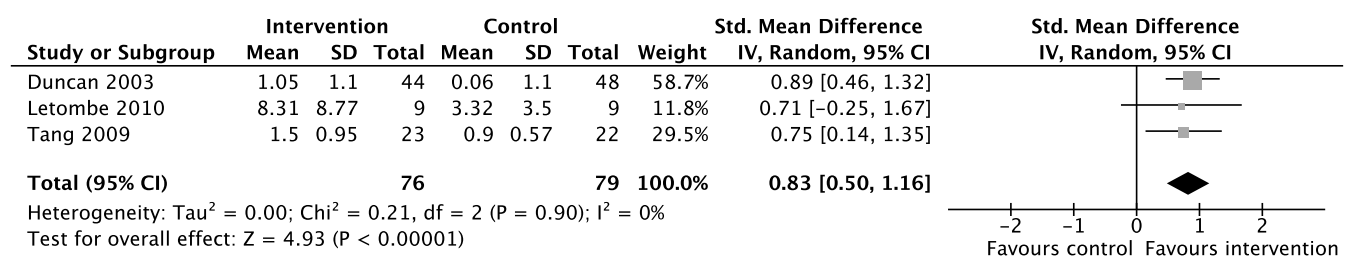

Figure 2.2: Forest plot of 3 trials comparing the effects of additional cardiovascular exercise on aerobic capacity in subacute stroke. Values are given in $\mathrm{mL} / \mathrm{min} / \mathrm{kg}$ peak oxygen uptake $\left(\dot{\mathrm{V}} \mathrm{O}_{2}\right.$ peak $)$. Abbreviations: $\mathrm{SD}=\mathrm{standard}$ deviation, $\mathrm{IV}=$ inverse variance, $\mathrm{CI}=$ confidence interval, $\mathrm{df}=$ degree of freedom.

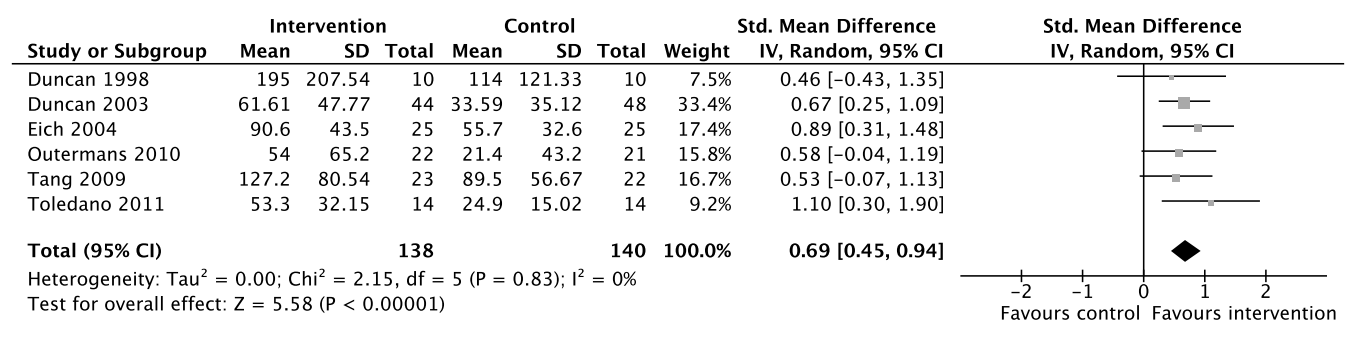

Figure 2.3: Forest plot of 6 trials comparing the effects of additional cardiovascular exercise on walking endurance using the 6 Minute Walk Test (6MWT) in subacute stroke. Values are given in maximal walking distance $(\mathrm{m})$ within 6 minutes. Abbreviations: $\mathrm{SD}=$ standard deviation, $\mathrm{IV}=$ inverse variance, $\mathrm{CI}=$ confidence interval, $\mathrm{df}=\mathrm{degrees}$ of freedom.

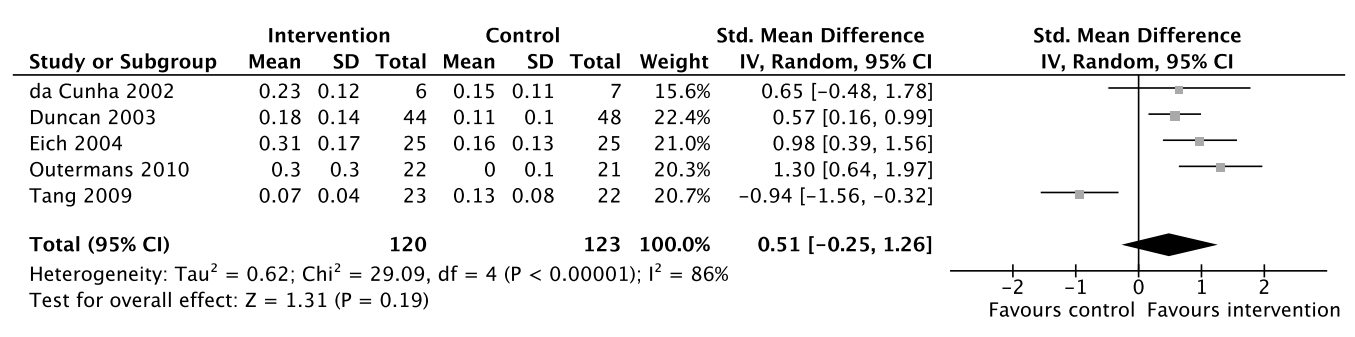

Figure 2.4: Forest plot of 5 trials comparing the effects of additional cardiovascular exercise on gait speed using the 10 Meter Walk Test (10MWT) in subacute stroke. Values are given in maximal gait speed (m/s) over 10 meters. Abbreviations: $\mathrm{SD}=$ standard deviation, $\mathrm{IV}=$ inverse variance, $\mathrm{CI}=$ confidence interval, $\mathrm{df}=$ degree of freedom. 


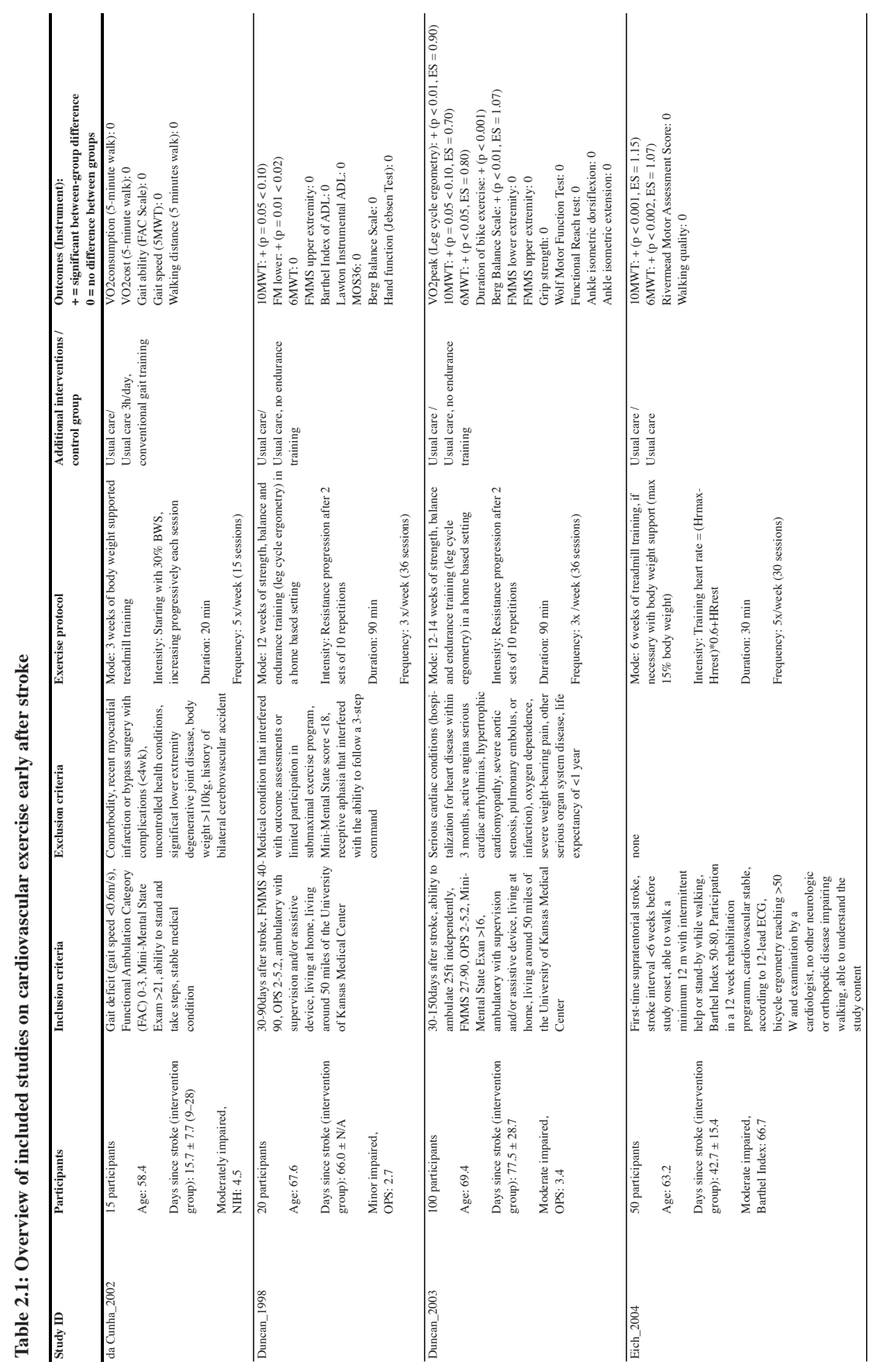




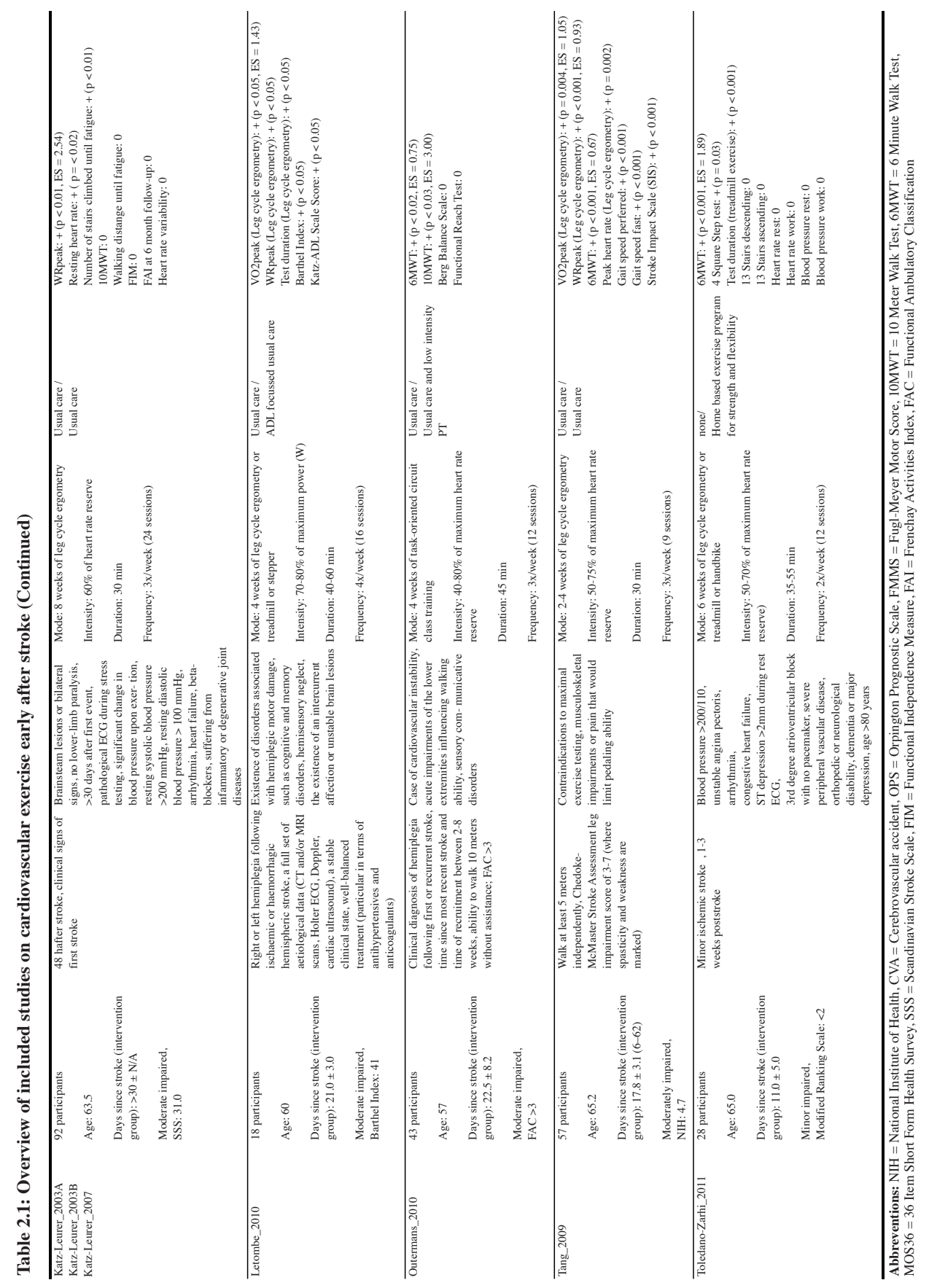




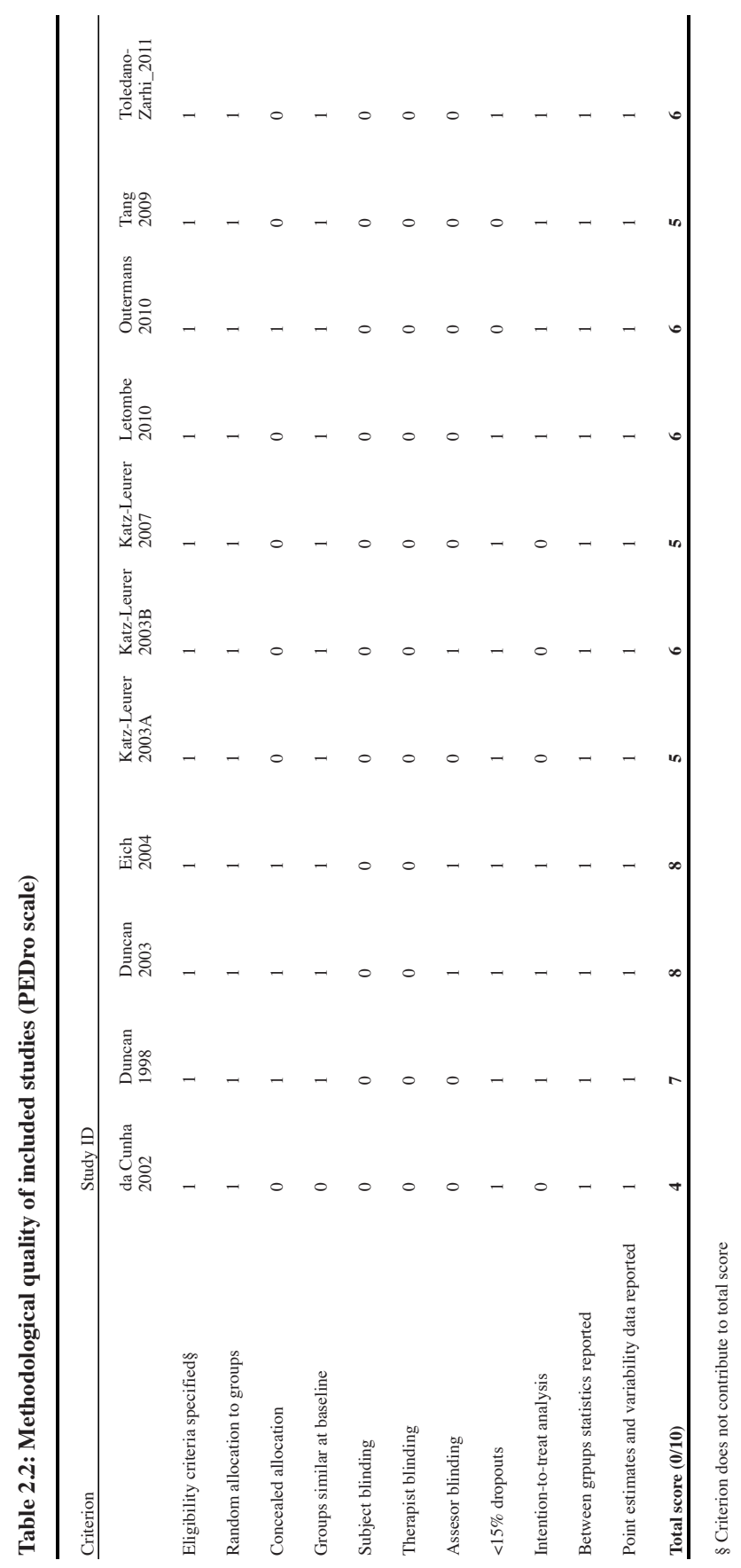




\section{Risk of bias across studies}

The syntheses of $\dot{\mathrm{V}} \mathrm{O}_{2}$ peak $\left(\mathrm{I}^{2}=0 \%, \mathrm{p}=0.90\right)$ and the $6 \mathrm{MWT}\left(\mathrm{I}^{2}=0 \%, \mathrm{p}=0.83\right)$ results were almost homogenous. We only detected significant heterogeneity for the outcome gait speed $\left(\mathrm{I}^{2}=\right.$ $86 \%, \mathrm{x}^{2}=29.09$, df $\left.=4, \mathrm{p}<0.01\right)$. The funnel plots revealed low publication bias in all of the 3 syntheses.

\section{Results of individual studies}

Three studies reported between-group improvements in $\dot{\mathrm{V}} \mathrm{O}_{2}$ peak after cardiovascular exercise $(\mathrm{ES}=0.90, \mathrm{MD}=0.99, \mathrm{CI} 95 \%=0.54-1.44)[34],(\mathrm{ES}=1.43, \mathrm{MD}=4.99, \mathrm{CI} 95 \%=-1.18-11.16)$ [39], $(\mathrm{ES}=1.05, \mathrm{MD}=0.60, \mathrm{CI} 95 \%=0.14-1.06)$ [41]. Katz-Leurer et al. [37] and Tang et al. [41] reported large effect sizes for Ppeak after cardiovascular exercise (ES = 2.54, MD = 11.00, CI95\% = -117.00-193.00) (ES = 0.93, MD = 4.00, CI95\% = 0.67-7.33), while Letombe et al. [39] did not publish baseline scores. Furthermore, Tang et al. reported a decrease in HRpeak after 24 weeks of cardiovascular exercise ( $\mathrm{ES}=-0.96, \mathrm{MD}=7.40, \mathrm{CI} 95 \%=-10.86-3.94$ ) compared to the control group [41]. In contrast, heart rate variability did not significantly decrease after cardiovascular exercise as reported by Katz-Leurer et al. [38]. The 6MWT values yielded betweengroup improvements in 6 studies after cardiovascular exercise intervention [33, 34, 35, 40, 41, 42], whereas da Cunha et al. assessed maximal walking distance using a 5-minute walk-test, and reported no between-group differences after 3 weeks of treadmill exercise training (ES $=1.21$, MD $=46.25$, CI95\% $=3.40-89.10$ ) [32]. Studies that used walking as a major part of the intervention protocol showed larger effect sizes in the 6MWT $(\mathrm{ES}=1.07, \mathrm{MD}=34.90, \mathrm{CI} 95 \%=13.59-56.21)$ [35], $(\mathrm{ES}=1.89, \mathrm{MD}=28.40, \mathrm{CI} 95 \%=9.81-46.99)$ [42] than studies that did not implement walking training $(\mathrm{ES}=0.67, \mathrm{MD}=81.00, \mathrm{CI} 95 \%=68.00-230.00)$ [33], $(\mathrm{ES}=0.80, \mathrm{MD}=28.02$, $\mathrm{CI} 95 \%=10.76-45.28)$ [34], $(\mathrm{ES}=0.67, \mathrm{MD}=37.70, \mathrm{CI} 95 \%=-2.85-78.25)$ [41]

Gait speed was increased in 4 of 5 studies after additional cardiovascular training [33, 34, 35, 40]. Tang et al. reported comparable improvements for both groups [41]. Motor recovery and functional ability were improved in 2 of 7 outcome measurements across 4 studies [33, 34, 39]. Duncan et al. reported improvements for the Fugl-Meyer Upper Extremity Score (ES = 1.68, MD $=6.20, \mathrm{CI} 95 \%=-2.85-15.25)$ and the Fugl-Meyer Lower Extremity Score $(\mathrm{ES}=21.0, \mathrm{MD}=5.67$, $\mathrm{CI} 95 \%=4.76-6.58)$ [33]. Letombe et al. reported improvements in ADL associated scores such as the Barthel-Index $(\mathrm{ES}=7.53, \mathrm{MD}=22.2, \mathrm{CI} 95 \%=4.86-39.54)$, and the Katz-ADL Scale (ES $=1.16, \mathrm{MD}=2.90, \mathrm{CI} 95 \%=1.39-4.41)$ [39] after cardiovascular exercise. Balance, measured in 3 studies, improved according to the Berg Balance Scale after 12 weeks of multimodal physical 
exercise training $(\mathrm{ES}=1.07, \mathrm{MD}=2.66, \mathrm{CI} 95 \%=1.43-3.89)$ [34]. A further 2 studies using the Berg Balance Scale or Functional Reach Test showed no improvements after physical exercise training $[33,34,40]$. Improved stair climbing and an associated decreased in fatigue was reported in one study by Katz-Leurer et al. [37]. In contrast to this is the finding of Toledano-Zarhi et al. who could not confirm these results based on measuring ascent and descent time of 13 steps [42]. Specific hand function assessed with the Wolf Motor Function Test [34] and the Jebsen Test [33] did not improve after 12 weeks of cardiovascular exercise. Grip strength showed no change after cardiovascular exercise in a study performed by Duncan et al. [34]. Finally, quality of life was measured and improved in 1 study using the Stroke Impact Scale (SIS) $(\mathrm{ES}=0.24, \mathrm{MD}=3.30$, CI95\% $=-5.38-11.98)$ [41].

\section{Adverse effects}

Few adverse events were reported during or after cardiovascular exercise in individuals with subacute stroke during the early rehabilitation phase. Recurrent stroke occurred in 3 out of 50 participants $(6 \%)$ in the intervention group during the cardiovascular exercise trial by Duncan et al.: 2 events occurred within 2 weeks after randomisation and one event occurred at seven weeks. No adverse events were reported during the training sessions [34]. Katz-Leurer et al. reported two cases that were withdrawn from a study due to myocardial infarction and deep vein thrombophlebitis, and two recurrent stroke attacks during follow up in the control group [36, 37]. Tang et al. reported exclusions due to acute low back pain, abnormal blood pressure responses, and abnormal electrocardiogram [41].

\section{Exercise testing protocol}

Four of 11 studies assessed aerobic capacity by spiro-ergometry or work rate evaluation using leg cycle ergometry; 2 of these studies reported the use of semi-recumbent bikes [34, 41], 1 study used wheelchair based ergometry [37], and 1 used standard leg cycle ergometry [38]. The protocols were similar in that resistance was increased between $510 \mathrm{~W}$ every 2 minutes and termination criteria defined according to ACSM were used [43]. However, there were large discrepancies regarding criteria for peak aerobic capacity. Duncan et al. [34] used a respiratory exchange ratio (RER) of $\geq 1.15$ and $90 \%$ of age predicted HR maximum (Formula N/A), Katz-Leurer et al. [37] used $85 \%$ of age predicted HR maximum $((220$-age $) \times 0.85))$, and Letombe et al. did not specify criteria for peak aerobic capacity [39]. Only Tang et al. [41] provided uniquely defined criteria such as a plateau in $\dot{\mathrm{V}} \mathrm{O}_{2}, \mathrm{RER} \geq 1.0$, and peak HR within 10 beats per minute of age predicted heart 
rate maximum $((208-(0.7 \times$ age $))$ as recommended by the ACSM guidelines for exercise testing and prescription [43]. There were no adverse effects reported during exercise testing in the studies reviewed.

\section{Discussion}

This systematic review provides an overview of the currently available evidence for the use of cardiovascular training in the early stages after stroke. The aim was to identify strategies that have the potential to influence aerobic capacity, physical functioning, and quality of life.

The methodological quality of the trials included was good ( 7 studies) to fair (4 studies). Failure to conceal the sequencing of treatment allocation before subject recruitment and failure to blind the outcome assessor were the most prevalent methodological shortcomings of the papers included.

The results of the meta-analysis for the $\dot{\mathrm{V}}{ }_{2}$ peak outcome after cardiovascular exercise, in individuals starting exercise within 6 days to 6 months after stroke onset, revealed a large effect size $(\mathrm{SMD}=0.83)$. This finding supports the evidence that individuals in the subacute stage after stroke have high potential to increase $\mathrm{VO}_{2}$ peak following a cardiovascular training intervention. This is in addition to a spontaneous recovery of peak aerobic capacity of $16.9 \%$ that occurs during the first 6 months after stroke, as shown by MacKay-Lyons \& Makrides [44]. The observed additional mean improvement in peak oxygen uptake $(+0.81 \mathrm{~mL} / \mathrm{min} / \mathrm{kg})$ after additional cardiovascular training in our meta-analysis seems to be low. But given the fact that $\dot{\mathrm{VO}_{2}}$ peak is reduced to $10-17 \mathrm{~mL} / \mathrm{min} / \mathrm{kg}$ within $0-30$ days after stroke [12, 13, 14], and $10 \mathrm{~mL} / \mathrm{min} / \mathrm{kg}$ is required for light instrumental activities during all activities of daily living (ADL) [45], this small improvement of peak aerobic capacity could lead to a large functional carryover. Furthermore, we have to consider that individuals with stroke need a higher aerobic capacity for basic ADL functions such as walking due to their impairments [46]. These facts confirm the importance of increasing aerobic capacity as much as possible. However, the short intervention lengths (mean 6.56 \pm 3.7 weeks) that were used in the included trials may be indicative of the fact that only small training effects were to be expected.

Ppeak, which is strongly related to $\dot{\mathrm{V}}{ }_{2}$ peak, revealed large effect sizes in 2 studies [37, 41]. However, these results may be strongly affected by the fact that leg cycle ergometry was used both as training intervention and test measure. Therefore, the observed effect might be partly attributable to learning effects and improved skill due to cycle practice. 
Consistent medium ES was found for walking distance within 6 minutes measured by the 6MWT. Detailed analysis revealed that individuals in the intervention group walked $31.68 \mathrm{~m}$ (CI95\%: 21.95-41.40) farther than participants without additional cardiovascular training. 3 of 6 studies either used treadmill exercise [35, 42] or focussed on walking competence [40] to improve cardiovascular fitness. Therefore, the latter study might also affect the 6MWT results by the additional gait practice that was performed. This might explain the lower ES in trials using leg cycle ergometry as the intervention method compared to trials using walking exercise. However, these significant improvements in walking distance after aerobic exercise using either a treadmill or walking based exercises demonstrate a functional carryover of enhanced aerobic capacity. Neither form of intervention was able to induce a change large enough to reach a minimal clinically important difference of $54.1 \mathrm{~m}$ during a 6 minute walk [47]. Whether this is due to insufficient training intensity, low training frequency, non-use of progression schemes, or other training related factors cannot be answered here. This should be a focal point of future trials where different training related parameters are compared; e.g. intensities, frequencies, and progression schemes.

Despite the fact that 4 out of 5 studies revealed moderate to large ES for improving gait speed, it remains unclear whether additional cardiovascular exercise can affect this outcome. The intervention group showed only a $0.1 \mathrm{~m} / \mathrm{s}$ faster gait speed compared to the control group, whereas $0.3 \mathrm{~m} / \mathrm{s}$ may be a minimal clinically relevant difference, as reported in a comparable sample of individuals with subacute stroke [47].

The outcomes motor recovery, functional ability and balance did not consistently improve after additional cardiovascular exercise. We found large heterogeneity in the outcome measurements that were used and, therefore, we were not able to synthesise these results. An improvement in motor recovery and functional ability after prolonged aerobic training, combined with the implementation of more sensitive measures, may lead to some evidence concerning the effects of cardiovascular exercise on physical function.

When the focus is put on functional activities, stair negotiation was reported in several studies. It remains unclear how the level of aerobic capacity influences the ability to ascend and descend stairs in subacute stroke. Katz-Leurer et al. have shown improvements in stair climbing until fatigue. However, this study failed to report baseline data for this parameter by only stating percentage improvements. In contrast, Toledano-Zarhi et al. could not report improvements in stair negotiation by measuring the time needed to negotiate 13 stairs while ascending and descending. Stair climbing depends on strength, balance and aerobic capacity, and is a rather complex motor task. What can be said is that if we are looking at aerobic capacity, stair-walking tests should 
have a longer duration than climbing 1 flight of stairs and reach symptom limited exhaustion of the participant.

In contrast to the findings of Ploughman et al. [48] derived from individuals with chronic stroke, upper-extremity tasks did not improve following additional cardiovascular exercise. Recent studies have shown that movement speed of the upper-extremities was increased after physical exercise training, probably due to an increase in core temperature and neuronal transmission [49]. A potentially biasing effect in this context could be that the tests were performed directly after the exercise, which could have influenced the test results. We therefore conclude that it is unclear whether cardiovascular exercise after stroke also has an impact on upper-extremity motor function.

Quality of life, evaluated by Tang et al. using the Stroke Impact Scale (SIS), revealed only low ES towards additional cardiovascular exercise [41]. This result might be explained by the short intervention length of 2-4 weeks. There were no other reports on this parameter from the other studies. However, the association of cardiovascular fitness, physical functioning and quality of life may be explored in further investigations, regarding the importance of carry over baseline effects into all ADL tasks.

Various exercise protocols have been used to improve cardiovascular fitness. Three studies used mixed training interventions [33, 34, 40] (strength, balance, endurance), and 2 studies did not explicitly exclude endurance training in the control group [32, 39, 42]. This inconsistent use of exercise protocols might lead to potential intervention bias regarding the evidence of optimal training protocols to be used in subacute stroke.

\section{Risk of cardiovascular exercise early after stroke}

Regarding the safety of early cardiovascular exercise after stroke, the incidence rate of adverse effects such as recurrent stroke reported in the analysed trials was comparable to the usual recurrent stroke risk, which is $8 \%$ between 1 to 6 months after stroke [50]. Further analysis based on the studies included yields a relative risk ratio of 1.25 for cardiovascular exercise compared to standard care following subacute stroke rehabilitation. Given the fact that cardiovascular training intervention started between 6 to 76 days (mean $34.28 \pm 25.1$ days) after stroke onset, and there is a low relative risk for adverse effects, it can be said that cardiovascular exercise early after stroke is safe. However, there were strict exclusion criteria in all studies, mostly based on the ACSM guidelines [43] (Table 2.1), thus individuals with stroke showing severe cardiac disease were not considered. Moreover, the applicability of cardiovascular exercise in the acute period after stroke $(\leq 7$ days post event) remains unclear. 


\section{Exercise testing early after stroke}

All exercise testing protocols used in the studies reviewed are consistent with the ACSM guidelines for exercise testing and prescription [43]. However, there is a lack of a precise description regarding termination criteria for exercise testing and evaluation of aerobic capacity (e.g. criteria for reaching $\dot{\mathrm{VO}}_{2}$ peak) in most of the included studies. Currently, leg cycle ergometry is the most appropriate and valid assessment method to measure aerobic capacity in individuals with lower fitness levels. None of the reviewed studies reported major adverse events that were directly attributable to the cardiovascular training. This can be regarded as an encouraging finding since it raises the possibility to start exercise training and testing in the very early period after stroke. It is important to note that participants tested in these studies were only moderately to mildly affected and certainly able to perform the established exercise testing methods such as semi-recumbent leg cycle ergometry. Concepts to evaluate aerobic capacity in severely affected individuals after stroke are lacking.

\section{Limitations}

We developed and utilised a structured study protocol to guide our search strategy, study selection, extraction of data and statistical analysis. Limitations of this review should however be noted. First, a publication bias may have been present, as well as a language bias, given that we considered only interventions described in published studies and restricted our search to English, French, and German language publications. Second, as there were few randomised trials, we also included one non-randomised study, the results of which may be affected by confounding bias due to the absence of random assignment. Third, as noted previously, participants included were moderately to mildly affected and, therefore, the results of the study are not fully generalisable to the stroke population at large. Furthermore, this analysis cannot provide statements concerning cardiovascular training effects in the very early period after stroke ( $\leq 7$ days post event). Fourth, reports of specific medication usage of the participants were not given in most studies; beta-blockers, for example, may affect cardiovascular responses during exercise [51]. Fifth, the relatively short duration of the interventions without follow-up for the course of outcomes after the cardiovascular exercise program might be considered a limitation. Sixth, the quality of the studies varied. Concealed allocation and the blinding of the assessors were not explicitly stated in the methodology or were absent. Seventh, the analyses of aerobic capacity strongly varied between studies or were not described sufficiently to ensure valid comparisons. Finally, publication bias was low but might account for some effects in the quantitative analysis. 


\section{Clinical relevance}

Regarding the results of the present meta-analysis, cardiovascular exercise interventions and exercise testing protocols using leg cycle ergometry have been found to be safe and feasible in the subacute stage after stroke. There is robust evidence that individuals with subacute stroke may benefit from these protocols to improve peak oxygen uptake and walking distance. Therefore, cardiovascular exercise protocols should be implemented into subacute stroke rehabilitation since conventional physical therapy programs between 2 and 14 weeks post-stroke did not elicit adequate cardiovascular stress to induce a training effect [13]. Clinicians and researchers should follow ACSM guidelines for exercise testing and prescription to ensure medical safety of training protocols and comparability for future analyses.

\section{Future directions}

Leg cycle ergometry or treadmill exercise has been shown to be adequate for moderately to mildly affected individuals with stroke, but these concepts are not applicable in severely affected individuals. Moreover, methods to assess aerobic capacity in the early post-stroke phase and in severely affected individuals with stroke are not available. Future research must develop new concepts for assessment of aerobic capacity in severely disabled individuals and for the early post-stroke period. It is well established that repetitions play a major role in structural reorganisation of the brain [52], and task-orientated activities are a key to functional recovery [53]. Therefore, cardiovascular exercise methods as well as exercise testing protocols must consider ADL-related functions such as walking or stair climbing as a means to influence and assess aerobic performance early after stroke. It is a future challenge to develop appropriate devices that operate with assistanceas-needed to challenge individuals optimally, by taking the quality of the individuals movements into account. Rehabilitation robotics could potentially support new directions in cardiovascular exercise testing and training early after stroke.

The effects of cardiovascular exercise on neuronal recovery, e.g. neuro-plasticity, recovery of function, cognition, etc., are still unknown. Future studies must address these gaps by designing study protocols using advanced assessments to evaluate the effects of cardiovascular exercise after stroke. Potential evidence in this field may justify the application of cardiovascular exercise protocols even for severely affected individuals.

The analysis of aerobic capacity is challenging since there are no standardised evaluation methods [54]. Future research should provide a consistent analysis process, even for individuals after stroke. 


\section{Conclusion}

This systematic review shows robust evidence that cardiovascular exercise early after stroke enhances aerobic capacity by improving $\dot{\mathrm{V}}{ }_{2}$ peak and walking distance during 6 minutes (6MWT) in moderately to mildly affected individuals. These findings have the potential for implementation in subacute stroke rehabilitation. Concepts to influence and evaluate aerobic capacity in severely affected individuals with subacute stroke, as well as in the very early period after stroke ( $\leq 7$ days post-stroke), are lacking. Further research is needed to develop appropriate methods for cardiovascular rehabilitation early after stroke, and to evaluate long-term effects of cardiovascular exercise on aerobic capacity, physical functioning, and quality of life.

\section{References}

[1] M. Koivusalo and M. Mackintosh, "The world health report 2007: A safer future: Global public health security in the 21st century," Development and Change, vol. 39, no. 6, pp. 1163-1169, 2008.

[2] T. Truelsen, B. Piechowski-Jozwiak, R. Bonita, C. Mathers, J. Bogousslavsky, and G. Boysen, "Stroke incidence and prevalence in europe: a review of available data," European Journal of Neurology, vol. 13, no. 6, pp. 581$598,2006$.

[3] W. Rosamond, K. Flegal, G. Friday, K. Furie, A. Go, K. Greenlund, N. Haase, M. Ho, V. Howard, B. Kissela, S. Kittner, D. Lloyd-Jones, M. McDermott, J. Meigs, C. Moy, G. Nichol, C. J. O’Donnell, V. Roger, J. Rumsfeld, P. Sorlie, J. Steinberger, T. Thom, S. Wasserthiel-Smoller, Y. L. Hong, and A. Amer Heart, "Heart disease and stroke statistics - 2007 update - a report from the american heart association statistics committee and stroke statistics subcommittee," Circulation, vol. 115, no. 5, pp. E69-E171, 2007.

[4] J. H. Carr, S. F. Mungovan, R. B. Shepherd, C. M. Dean, and L. A. Nordholm, "Physiotherapy in stroke rehabilitation: bases for australian physiotherapists' choice of treatment," Physiotherapy Theory and Practice, vol. 10, no. 4, pp. 201-209, 1994.

[5] D. Wade, "Rehabilitation therapy after stroke," The Lancet, vol. 354, no. 9174, pp. 176-177, 1999.

[6] E. J. Roth, A. W. Heinemann, L. L. Lovell, R. L. Harvey, J. R. McGuire, and S. Diaz, "Impairment and disability: their relation during stroke rehabilitation," Archives of physical medicine and rehabilitation, vol. 79, no. 3, pp. 329-329, 1998.

[7] E. J. Roth, "Heart disease in patients with stroke: incidence, impact, and implications for rehabilitation. part 1: Classification and prevalence," Archives of Physical Medicine and Rehabilitation, vol. 74, no. 7, pp. 752-760, 1993.

[8] E. J. Roth, "Heart disease in patients with stroke. part 2: Impact and implications for rehabilitation," Archives of Physical Medicine and Rehabilitation, vol. 75, no. 1, pp. 94-101, 1994.

[9] G. E. Gresham, T. F. Phillips, P. A. Wolf, P. M. McNamara, W. B. Kannel, and T. R. Dawber, "Epidemiologic profile of long-term stroke disability: the framingham study," Archives of Physical Medicine and Rehabilitation, vol. 60, no. 11, pp. 487-491, 1979. 
[10] C. E. Hafer-Macko, A. S. Ryan, F. M. Ivey, and R. F. Macko, "Skeletal muscle changes after hemiparetic stroke and potential beneficial effects of exercise intervention strategies," Journal of Rehabilitation Research and Development, vol. 45, no. 2, pp. 261-272, 2008.

[11] K. Potempa, M. Lopez, L. T. Braun, J. P. Szidon, L. Fogg, and T. Tincknell, "Physiological outcomes of aerobic exercise training in hemiparetic stroke patients," Stroke, vol. 26, no. 1, pp. 101-105, 1995.

[12] J. O. Kelly, S. L. Kilbreath, G. M. Davis, B. Zeman, and J. Raymond, "Cardiorespiratory fitness and walking ability in subacute stroke patients," Archives of Physical Medicine Rehabilitation, vol. 84, no. 12, pp. 1780$1785,2003$.

[13] M. J. MacKay-Lyons and L. Makrides, "Cardiovascular stress during a contemporary stroke rehabilitation program: Is the intensity adequate to induce a training effect?," Archives of Physical Medicine and Rehabilitation, vol. 83, no. 10, pp. 1378-1383, 2002.

[14] A. Tang, K. M. Sibley, S. G. Thomas, W. E. McIlroy, and D. Brooks, "Maximal exercise test results in subacute stroke," Archives of Physical Medicine and Rehabilitation, vol. 87, no. 8, pp. 1100-1105, 2006.

[15] D. Brooks, A. Tang, K. M. Sibley, and W. E. McIlroy, "Profile of patients at admission into an inpatient stroke rehabilitation programme: cardiorespiratory fitness and functional characteristics," Physiotherapy Canada, vol. 60, no. 2 , pp. $171-179,2008$.

[16] J. J. Eng, A. S. Dawson, and K. S. Chu, "Submaximal exercise in persons with stroke: Test-retest reliability and concurrent validity with maximal oxygen consumption," Archives of Physical Medicine and Rehabilitation vol. 85, no. 1, pp. 113-118, 2004.

[17] J. Ramas, A. Courbon, F. Roche, F. Bethoux, and P. Calmels, "Effect of training programs and exercise in adult stroke patients: literature review," Annales de Readaptation et de Medecine Physique, vol. 50, no. 6, pp. 438444, 2007.

[18] F. M. Ivey, R. F. Macko, A. S. Ryan, and C. E. Hafer-Macko, "Cardiovascular health and fitness after stroke," Topics in Stroke Rehabilitation, vol. 12, no. 1, pp. 1-16, 2005.

[19] E. J. Roth, K. Mueller, and D. Green, “Stroke rehabilitation outcome - impact of coronary-artery disease,” Stroke, vol. 19 , no. 1 , pp. 42-47, 1988.

[20] A. Bateman, F. J. Culpan, A. D. Pickering, J. H. Powell, O. M. Scott, and R. J. Greenwood, "The effect of aerobic training on rehabilitation outcomes after recent severe brain injury: A randomized controlled evaluation," Archives of Physical Medicine and Rehabilitation, vol. 82, no. 2, pp. 174-182, 2001.

[21] K. S. Chu, J. J. Eng, A. S. Dawson, J. E. Harris, A. Ozkaplan, and S. Gylfadottir, "Water-based exercise for cardiovascular fitness in people with chronic stroke: a randomized controlled trial," Archives of physical medicine and rehabilitation, vol. 85, no. 6, pp. 870-870, 2004.

[22] J. J. Eng, K. S. Chu, C. M. Kim, A. S. Dawson, A. Carswell, and K. E. Hepburn, "A community-based group exercise program for persons with chronic stroke," Medicine Science in Sports Exercise, vol. 35, no. 8, pp. 1271$1278,2003$.

[23] C. Globas, C. Becker, J. Cerny, J. M. Lam, U. Lindemann, L. W. Forrester, R. F. Macko, and A. R. Luft, "Chronic stroke survivors benefit from high-intensity aerobic treadmill exercise: a randomized control trial," Neurorehabil Neural Repair, vol. 26, no. 1, pp. 85-95, 2012.

[24] F. M. Ivey, C. E. Hafer-Macko, and R. F. Macko, "Task-oriented treadmill exercise training in chronic hemiparetic stroke," Journal of Rehabilitation Research and Development, vol. 45, no. 2, pp. 249-259, 2008.

[25] R. F. Macko, F. M. Ivey, L. W. Forrester, D. Hanley, J. D. Sorkin, L. I. Katzel, K. H. Silver, and A. P. Goldberg, "Treadmill exercise rehabilitation improves ambulatory function and cardiovascular fitness in patients with chronic stroke - a randomized, controlled trial," Stroke, vol. 36, no. 10, pp. 2206-2211, 2005. 
[26] M. Y. C. Pang, J. J. Eng, A. S. Dawson, H. A. McKay, and J. E. Harris, “A community-based fitness and mobility exercise program for older adults with chronic stroke: a randomized, controlled trial," Journal of the American Geriatrics Society, vol. 53, no. 10, pp. 1667-1674, 2005.

[27] M. Y. C. Pang, J. J. Eng, A. S. Dawson, and S. Gylfadottir, "The use of aerobic exercise training in improving aerobic capacity in individuals with stroke: a meta-analysis," Clinical Rehabilitation, vol. 20, no. 2, pp. 97-111, 2006.

[28] H. S. Jorgensen, H. Nakayama, H. O. Raaschou, and T. S. Olsen, "Recovery of walking function in stroke patients: the copenhagen stroke study," Arch Phys Med Rehabil, vol. 76, no. 1, pp. 27-32, 1995.

[29] C. G. Maher, C. Sherrington, R. D. Herbert, A. M. Moseley, and M. Elkins, "Reliability of the pedro scale for rating quality of randomized controlled trials," Physical Therapy, vol. 83, no. 8, pp. 713-721, 2003.

[30] J. R. Landis and G. G. Koch, "The measurement of observer agreement for categorical data," Biometrics, vol. 33 , no. 1, pp. 159-174, 1977.

[31] A. Liberati, D. G. Altman, J. Tetzlaff, C. Mulrow, P. C. Gotzsche, J. P. A. Ioannidis, M. Clarke, P. J. Devereaux, J. Kleijnen, and D. Moher, "The prisma statement for reporting systematic reviews and meta-analyses of studies that evaluate healthcare interventions: explanation and elaboration," British Medical Journal, vol. 339, p. b2700, 2009 .

[32] I. T. da Cunha, P. A. Lim, H. Qureshy, H. Henson, T. Monga, and E. J. Protas, "Gait outcomes after acute stroke rehabilitation with supported treadmill ambulation training: A randomized controlled pilot study," Archives of Physical Medicine and Rehabilitation, vol. 83, no. 9, pp. 1258-1265, 2002.

[33] P. Duncan, L. Richards, D. Wallace, J. Stoker-Yates, P. P. Pohl, C. Luchies, A. Ogle, and S. Studenski, "A randomized, controlled pilot study of a home-based exercise program for individuals with mild and moderate stroke," Stroke, vol. 29, no. 10, pp. 2055-2060, 1998.

[34] P. Duncan, S. Studenski, L. Richards, S. Gollub, S. M. Lai, D. Reker, S. Perera, J. Yates, V. Koch, S. Rigler, and D. Johnson, "Randomized clinical trial of therapeutic exercise in subacute stroke," Stroke, vol. 34, no. 9, pp. 2173-2180, 2003.

[35] H. J. Eich, H. Mach, C. Werner, and S. Hesse, "Aerobic treadmill plus bobath walking training improves walking in subacute stroke: a randomized controlled trial," Clinical Rehabilitation, vol. 18, no. 6, pp. 640-651, 2004.

[36] M. Katz-Leurer, E. Carmeli, and M. Shochina, "The effect of early aerobic training on independence six months post stroke," Clinical Rehabilitation, vol. 17, no. 7, pp. 735-741, 2003.

[37] M. Katz-Leurer, M. Shochina, E. Carmeli, and Y. Friedlander, "The influence of early aerobic training on the functional capacity in patients with cerebrovascular accident at the subacute stage," Archives of Physical Medicine and Rehabilitation, vol. 84, no. 11, pp. 1609-1614, 2003.

[38] M. Katz-Leurer and M. Shochina, "The influence of autonomic impairment on aerobic exercise outcome in stroke patients," NeuroRehabilitation, vol. 22, no. 4, pp. 267-272, 2007.

[39] A. Letombe, C. Cornille, H. Delahaye, A. Khaled, O. Morice, A. Tomaszewski, and N. Olivier, "Early poststroke physical conditioning in hemiplegic patients: A preliminary study," Annals of Physical Rehabilitation Medicine, vol. 53, no. 10, pp. 632-642, 2010.

[40] J. C. Outermans, R. P. S. van Peppen, H. Wittink, T. Takken, and G. Kwakkel, "Effects of a high-intensity taskoriented training on gait performance early after stroke: a pilot study," Clinical Rehabilitation, vol. 24, no. 11, pp. 979-987, 2010.

[41] A. Tang, K. M. Sibley, S. G. Thomas, M. T. Bayley, D. Richardson, W. E. McIlroy, and D. Brooks, "Effects of an aerobic exercise program on aerobic capacity, spatiotemporal gait parameters, and functional capacity in subacute stroke," Neurorehabilitation and Neural Repair, vol. 23, no. 4, pp. 398-406, 2009. 
[42] A. Toledano-Zarhi, D. Tanne, E. Carmeli, and M. Katz-Leurer, "Feasibility, safety and efficacy of an early aerobic rehabilitation program for patients after minor ischemic stroke: A pilot randomized controlled trial," Neurorehabilitation, vol. 28, no. 2, pp. 85-90, 2011.

[43] W. R. Thompson, N. F. Gordon, and L. S. Pescatello, American College of Sports Medicine. Guidelines for Exercise Testing and Prescription. Philadelphia: Lippincott Williams Wilkins, eight ed., 2010.

[44] M. J. MacKay-Lyons and L. Makrides, "Longitudinal changes in exercise capacity after stroke," Archives of Physical Medicine and Rehabilitation, vol. 85, no. 10, pp. 1608-1612, 2004.

[45] F. M. Ivey, C. E. Hafer-Macko, and R. F. Macko, “Exercise rehabilitation after stroke," NeuroRx, vol. 3, no. 4, pp. 439-450, 2006.

[46] A. Danielsson and K. S. Sunnerhagen, "Oxygen consumption during treadmill walking with and without body weight support in patients with hemiparesis after stroke and in healthy subjects," Archives of Physical Medicine Rehabilitation, vol. 81, no. 7, pp. 953-957, 2000.

[47] G. D. Fulk, C. Reynolds, S. Mondal, and J. E. Deutsch, "Predicting home and community walking activity in people with stroke," Archives of Physical Medicine and Rehabilitation, vol. 91, no. 10, pp. 1582-1586, 2010.

[48] M. Ploughman, J. McCarthy, M. Bosse, H. J. Sullivan, and D. Corbett, "Does treadmill exercise improve performance of cognitive or upper-extremity tasks in people with chronic stroke? a randomized cross-over trial,' Archives of Physical Medicine and Rehabilitation, vol. 89, no. 11, pp. 2041-2047, 2008.

[49] T. McMorris, K. Collard, J. Corbett, M. Dicks, and J. P. Swain, "A test of the catecholamines hypothesis for an acute exercise-cognition interaction,” Pharmacology Biochemistry and Behavior, vol. 89, no. 1, pp. 106-115, 2008.

[50] K. Hardie, G. J. Hankey, K. Jamrozik, R. J. Broadhurst, and C. Anderson, "Ten-year risk of first recurrent stroke and disability after first-ever stroke in the perth community stroke study," Stroke, vol. 35, no. 3, pp. 731-735, 2004.

[51] P. A. Tesch, “Exercise performance and beta-blockade," Sports Medicine, vol. 2, no. 6, pp. 389-412, 1985.

[52] M. P. Kilgard and M. M. Merzenich, "Cortical map reorganization enabled by nucleus basalis activity," Science, vol. 279, no. 5357, pp. 1714-1718, 1998.

[53] B. French, L. H. Thomas, M. J. Leathley, C. J. Sutton, J. McAdam, A. Forster, P. Langhorne, C. I. M. Price, A. Walker, and C. L. Watkins, "Repetitive task training for improving functional ability after stroke," Cochrane Database of Systematic Reviews, no. 4, p. CD006073, 2007.

[54] R. A. Robergs, D. Dwyer, and T. Astorino, "Recommendations for improved data processing from expired gas analysis indirect calorimetry," Sports Medicine, vol. 40, no. 2, pp. 95-111, 2010. 


\title{
Chapter 3
}

\section{Evaluation of exercise capacity after severe stroke using robotics-assisted treadmill exercise: a proof-of-concept study}

Technology and Health Care 2013;21(2):157-166

doi: 10.3233/THC-130730

\author{
Oliver Stoller \\ Eling D de Bruin \\ Matthias Schindelholz \\ Corina Schuster \\ Rob A de Bie \\ Kenneth J Hunt
}




\section{Abstract}

Background: Robotics-assisted treadmill exercise (RATE) with focus on motor recovery has become popular in early post-stroke rehabilitation but low endurance for exercise is highly prevalent in these individuals. This study aimed to develop an exercise testing method using robotics-assisted treadmill exercise to evaluate aerobic capacity after severe stroke.

Methods: Constant load testing (CLT) based on body weight support (BWS) control, and incremental exercise testing (IET) based on guidance force (GF) control were implemented during RATE. Analyses focussed on step change, step response kinetics, and peak performance parameters of oxygen uptake.

Results: Three subjects with severe motor impairment 16-23 days post-stroke were included. CLT yielded reasonable step change values in oxygen uptake, whereas response kinetics of oxygen uptake showed low goodness of fit. Peak performance parameters were not obtained during IET.

Conclusion: Exercise testing in post-stroke individuals with severe motor impairments using a BWS control strategy for CLT is deemed feasible and safe. Our approach yielded reasonable results regarding cardiovascular performance parameters. IET based on GF control does not provoke peak cardiovascular performance due to uncoordinated walking patterns. GF control needs further development to optimally demand active participation during RATE. The findings warrant further research regarding the evaluation of exercise capacity after severe stroke. 


\section{Background}

Robotics-assisted treadmill exercise (RATE) has become popular for enhancement of recovery early after stroke $[1,2,3,4,5,6,7]$. Most research efforts in the field of assistive rehabilitation robotics for the lower extremities have focussed on motor recovery, e.g. the restoration of walking function. However, around $75 \%$ of individuals post-stroke suffer from cardiac disease [8], and the majority have low endurance for exercise as a secondary reaction to immobility [9, 10]. RATE might be used to provoke cardiovascular responses and to introduce and guide cardiovascular rehabilitation in individuals with severe motor impairment after stroke.

A recent meta-analysis revealed that cardiovascular exercise is safe and effective in improving peak oxygen uptake and walking distance in the subacute stages after stroke (6 days - 6 months post stroke) [11]. Considering that routine neuro-rehabilitation programs did not elicit adequate cardiovascular stress to induce a training effect [12], new concepts for cardiovascular rehabilitation early after stroke are required, because severely impaired, non-ambulatory individuals after stroke cannot benefit from conventional exercise.

The introduction of RATE, in addition to be a promising treatment option, might be used for exercise testing in this population. RATE facilitates the introduction of treadmill walking early after stroke and offers potential for exercise testing even for individuals with severe motor impairment. Considering that most motor recovery occurs in the first 3 months [13], the implementation of a functional task (e.g. walking) may be important with regard to the training intensity necessary to achieve cardiovascular benefits. This concept allows exploration of the effects of early cardiovascular exercise on aerobic capacity, motor recovery, cognition, and quality of life.

Initial work showed that passive RATE provokes an increase in oxygen uptake $[14,15]$. Further experimental studies exploring novel assessment protocols to estimate key cardiovascular performance parameters directly, by using feedback-controlled RATE, have shown feasibility and effectiveness in clinical settings in non-ambulatory individuals after spinal cord injury $[16,17]$ and stroke [18]. A human-in-the-loop feedback control mechanism was incorporated which allowed individuals to maximise their voluntary effort and associated cardiovascular stress during RATE [19]. The underlying assumption driving this research was that this method might offer the possibility to evaluate aerobic capacity and guide cardiovascular rehabilitation in non-ambulatory individuals early after the event. However, a number of individuals are not able to produce adequate voluntary effort within the exoskeleton in order to increase cardiovascular stress during RATE due to poor motor status. We hypothesise that active participation strategies, based on progressive reduction of body weight support and robotics-assistance, might have the potential to provoke cardiovas- 
cular responses from these individuals. Therefore, we designed novel protocols to estimate key cardiovascular performance parameters for post-stroke individuals with severe motor impairments using (1) a body weight support (BWS) control strategy for constant load testing (CLT), and (2) a guidance force (GF) control strategy for incremental exercise testing (IET).

The purpose of this proof-of-concept study was to explore these two novel strategies for provoking a cardiovascular response and to evaluate aerobic capacity on a single case basis. This study aimed (1) to develop an exercise testing method based on principles of exercise physiology to provoke cardiovascular responses and to evaluate aerobic capacity in post-stroke individuals with severe motor impairments, (2) to assess whether this new method would lead to acquisition of usable cardiovascular performance data based on international guidelines for exercise testing and prescription, (3) to evaluate the feasibility and safety of the procedure, and (4) to propose future research directions for exercise testing early after stroke.

\section{Methods}

\section{Subjects}

In-patients after stroke were recruited from a midsize rehabilitation centre in the Northwestern part of Switzerland. Inclusion criteria were (1) a clinical diagnosis of first-ever ischaemic stroke, (2) $\leq 4$ weeks post stroke, (3) age $\geq 18$ years, (4) Functional Ambulation Classification (FAC) $\leq 1$, (5) stable medical condition, and (6) Mini Mental State Examination (MMSE) score $\geq 24$. Subjects were excluded if they had (a) cardiac contraindications for exercise testing [20], and (b) contraindications for RATE according to guidelines from the manufacturer of the device. All subjects were informed about risks and benefits, and gave signed informed consent. The Ethics Review Committee of Canton Aargau, Switzerland, approved the study.

\section{Instrumentation}

We used the Lokomat system (Hocoma AG, Volketswil, Switzerland) with an integrated treadmill (h/p/cosmos sports and medical GmbH, Traunstein, Germany) for RATE. The exoskeleton provides active control at the hip and knee joints of both legs using DC motors, whereas passive foot lifters support dorsiflexion of the ankles during the swing phase. Aerobic capacity was assessed using breath-by-breath spirometry (MetaMax 3B, Cortex Biophysik, Leipzig, Germany) calibrated prior to each test according to the manufacturers specifications. Heart rate (HR) was measured 
continually by a wireless sender belt (T31, Polar Electro, Kempele, Finland) and a receiver board (HRMI, Sparkfun, Boulder, USA).

BWS was implemented using a dynamic body weight unloading system, consisting of an elastic spring element to take over the main unloading force and an active closed-loop controlled electric drive to generate the exact desired force (Lokolift system, Hocoma AG, Volketswil, Switzerland). A significant reduction in BWS requires additional activity of the subjects; this fact is used to generate a step increase in exercise intensity for CLT.

GF controlled IET was implemented using reduction of the Lokomats guidance force, which is based on progressive torque reduction of the hip and knee joints. Reducing GF progressively requires increased walking activity of the subjects to obtain the defined movement pattern; this fact is used to provoke incremental workloads for IET.

\section{Experimental protocol}

The protocol consisted of 2 familiarisation sessions, 2 CLT (T1, T2), and 2 IET (T3, T4) completed in the space of 2 days. Familiarisation focussed on adjusting the system to provide a physiological gait pattern and to ensure that the subjects could walk comfortably in the exoskeleton. Initially, the minimal body weight support (BWSmin) was evaluated by implementing a progressive reduction of $10 \%$ in BWS each minute until the subject was unable to follow a physiological gait pattern, defined as (1) adequate heel strike, (2) no dragging during the swing phase, (3) weight-bearing during the stance phase of both legs. Treadmill speed was fixed between $1.8-2 \mathrm{~km} / \mathrm{h}$, depending on the subjects height. Blood pressure was checked prior to all tests in a sitting position and every three minutes while performing the tests. There was close adherence to guidelines for exercise testing [20].

For CLT, the protocol consisted of (1) rest for 5 minutes with $100 \%$ BWS, (2) walking with $80 \%$ BWS for 5 minutes, (3) walking with BWSmin for 10 minutes, (4) walking with $80 \%$ BWS for 5 minutes, (5) rest for 5 minutes with 100\% BWS (Figure 3.1A). IET consisted of 10 minutes incremental exercise by implementing the GF reduction protocol. The tests consisted of (1) walking with BWSmin for 5 minutes, (2) walking with BWSmin for 10 minutes while GF was reduced by $10 \%$ each minute, (3) walking with BWSmin for 5 minutes (Figure 3.1B).

Termination criteria for all tests were: (a) abnormal blood pressure response, i.e. hypertensive (systolic $\mathrm{BP} \geq 210 \mathrm{mmHg}$ or diastolic $\mathrm{BP} \geq 115 \mathrm{mmHg}$ ), or hypotensive responses (decrease in $\mathrm{BP} \geq 10 \mathrm{mmHg}$ ), (b) peak heart rate $\geq 85 \%$ of age-predicted heart rate maximum [208-(0.7 $\mathrm{x}$ age $)$ ] [21], (c) respiratory exchange ratio (RER) of $\geq 1.15$, (d) obvious inability to follow a physiological gait pattern, (e) pain or discomfort in the chest. 


\section{Outcomes}

Outcome measures for CLT were step response kinetics of oxygen uptake (time constants during on-/off transition), and steady-state change in oxygen uptake $\left(\Delta \dot{\mathrm{V}} \mathrm{O}_{2}\right)$, heart rate $(\Delta \mathrm{HR})$, respiratory volume $\left(\Delta \dot{V}_{E}\right)$, and respiratory rate $\left(\Delta R_{f}\right)$. IET focussed on peak performance parameters such as peak oxygen uptake $\left(\mathrm{V}_{2}\right.$ peak), peak respiratory exchange ratio (RERpeak), gas exchange threshold (GET), peak heart rate (HRpeak), peak respiratory volume ( $\dot{\mathrm{V}}_{\text {Epeak }}$ ), and peak respiratory rate $\left(\mathrm{R}_{\mathrm{f}}\right.$ peak). Criteria for success were subjects compliance to the experimental protocol, clinical safety, cardiovascular response to exercise testing using RATE, and successful data processing.

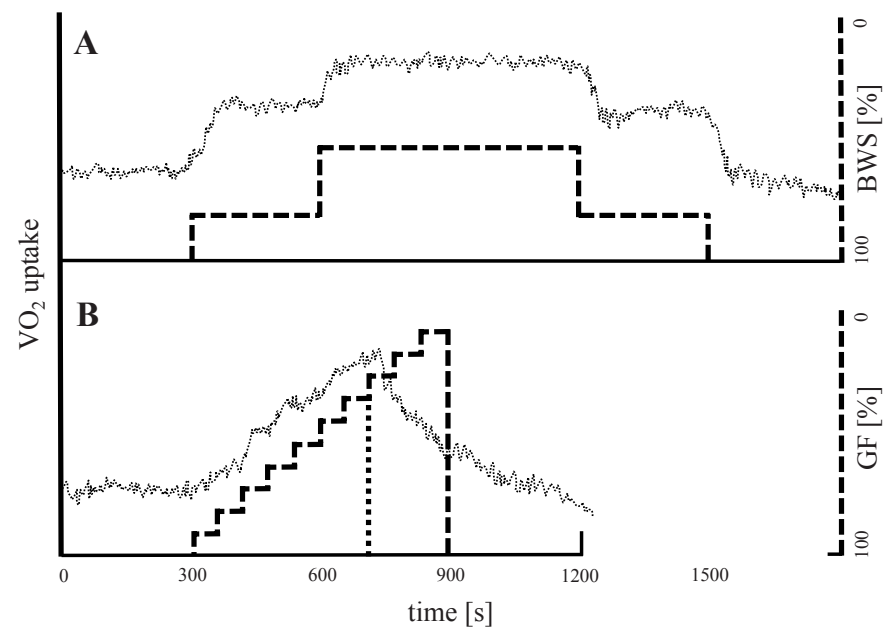

Figure 3.1: Schematic representation of constant load testing (CLT) based on a body weight support (BWS) control strategy (A), and incremental exercise testing (IET) based on a guidance force (GF) control strategy (B). The dotted thick line represents a potential termination stage during IET.

\section{Analysis}

Descriptive analyses were performed on all variables. Raw breath-by-breath data were processed using a bias free moving average filter over 15 breath [22]. Outliers beyond 2 standard deviations between raw and filtered data were removed. Time constants of oxygen uptake kinetics were calculated using a non-linear least-squares algorithm to fit the data as described in the following 
mono-exponential equation: $\dot{\mathrm{VO}}_{2}(\mathrm{t})=\dot{\mathrm{V}} \mathrm{O}_{2}(\mathrm{~b})+\dot{\mathrm{V}} \mathrm{O}_{2}\left(1-\mathrm{e}^{-(\mathrm{t}-\mathrm{Td}) / \tau}\right), \mathrm{t}<0$ with $\dot{\mathrm{VO}}_{2}(\mathrm{~b})=\dot{\mathrm{V}} \mathrm{O}_{2}$ at baseline, $\Delta \dot{\mathrm{VO}}_{2}=$ step increase in oxygen uptake, $\mathrm{Td}=$ time delay of $20 \mathrm{~s}$, and $\tau=$ time constant. $\mathrm{R}^{2}$ values were computed to describe goodness of fit of the non-linear least-squares algorithm. To calculate changes between testing stages, steady state was defined as the mean value of the last 3 minutes during every interval for all outcome measures. $\dot{\mathrm{V}} \mathrm{O}_{2}$ peak was defined as the mean $\dot{\mathrm{V}} \mathrm{O}_{2}$ in the last 20 seconds during incremental exercise, whereas RERpeak, HRpeak, $\dot{V}_{\text {Epeak, and }}$ $\mathrm{R}_{\mathrm{f}}$ peak were defined as the maximal values reached during IET. All analyses and displays were performed using MATLAB (Version R2010a, MathWorks, Natick MA, USA).

\section{Results}

Three subjects were included (Table 3.1). The subjects were similar with regard to lesion, affected body side, time post stroke onset, and FAC.

During CLT, all subjects reached a BWSmin level of 50\%. Time constants of oxygen uptake kinetics for rest/passive and passive/rest steps during CLT were reasonable, but yielded low goodness of fit $\left(\mathrm{R}^{2}\right)$ in most of the analyses. Step response kinetics for passive/active and active/passive steps were inconsistent throughout. Subjects generally yielded higher oxygen uptake values from rest to passive walking (mean $\pm \mathrm{SD} \Delta \dot{\mathrm{V}} \mathrm{O}_{2}=3.2 \pm 2.1 \mathrm{~mL} / \mathrm{min} / \mathrm{kg}$ ) than from passive to active walking condition $\left(\Delta \dot{\mathrm{V}} \mathrm{O}_{2} \quad 1.5 \pm 0.8 \mathrm{~mL} / \mathrm{min} / \mathrm{kg}\right.$ ) (Figure 3.2). The step change $\dot{\mathrm{V}} \mathrm{O}_{2}$ was associated with an increase in $\dot{\mathrm{V}}_{\mathrm{E}}$ (rest to passive $\Delta \dot{\mathrm{V}}_{\mathrm{E}}=5.3 \pm 3.0 \mathrm{~L} / \mathrm{min}$, passive to active $\Delta \dot{\mathrm{V}}_{\mathrm{E}}=3.2 \pm 1.7 \mathrm{~L} / \mathrm{min}$ ) during all CLT stages. However, $\mathrm{HR}$ (rest to passive $\Delta \mathrm{HR}=7.7 \pm 13.3$ beats $/ \mathrm{min}$, passive to active $\Delta \mathrm{HR}=3.6 \pm 7.8$ beats $/ \mathrm{min}$ ) and $\mathrm{R}_{\mathrm{f}}$ (rest to passive $\Delta \mathrm{R}_{\mathrm{f}}=1.7 \pm 3.8$ beats $/ \mathrm{min}$, passive to active $\Delta \mathrm{R}_{\mathrm{f}}=1.0 \pm 3.4$ beats $/ \mathrm{min}$ ) did not increase consistently.

For IET, the lowest achieved GF level (GFmin) ranged between $40-10 \%$, and $\dot{\mathrm{VO}}_{2}$ peak values were mean \pm SD $10.6 \pm 3.7 \mathrm{~mL} / \mathrm{min} / \mathrm{kg}$ (Figure 3.3). RER values $(0.9 \pm 0.1)$ were always below the termination threshold $(\geq 1.15)$. GET could not be analysed due to inconsistent progression of breath-by-breath values. The $85 \%$ threshold of age predicted HRpeak values was not reached in any subject. HR (change $14.2 \pm 9.3$ beats/min), $\dot{\mathrm{V}}_{\mathrm{E}}$ (change $7.2 \pm 3.0 \mathrm{~L} / \mathrm{min}$ ), and $\mathrm{R}_{\mathrm{f}}$ (change $20.8 \pm 3.3$ beats/min) increased in all of the subjects.

The procedure has found to be feasible and safe for individuals with severe motor impairment early after stroke. All subjects completed the four tests without any safety concerns. Subjects showed overall compliance, whereas data recording and processing during the experimental setup was successful for all procedures. 


\begin{tabular}{llll} 
& Subject 1 & Subject 2 & Subject 3 \\
Sex & Female & Male & Female \\
Age $(\mathrm{yr})$ & 60 & 54 & 71 \\
Body mass $(\mathrm{kg})$ & 58 & 93 & 64 \\
Body height $(\mathrm{cm})$ & 164 & 192 & 155 \\
BMI $\left(\mathrm{kg} / \mathrm{m}^{2}\right)$ & 22 & 25 & 27 \\
Diagnosis & Ischemic stroke & Ischemic stroke & Ischemic stroke \\
Affected body side & Right & Right & Right \\
Days post event (d) & 16 & 23 & 16 \\
Medication & $1,2,3,4,5$ & $1,3,4,6$ & $1,3,4,6,7$ \\
MMSE (0/30) & 29 & 27 & 24 \\
FAC (0/5) & 0 & 0 & 0 \\
EBI at clinical admission (0/64) & 40 & 34 & 18 \\
EBI during experiment (0/64) & 41 & 34 & 18 \\
EBI at clinical discharge (0/64) & 55 & 49 & 23 \\
& \multicolumn{2}{l}{} \\
\hline Abbreviations: BMI = Body Mass Index, MMSE = Mini Mental State Examination, FAC = Functional Ambulation \\
Classification, EBI = Extended Barthel Index, $1=$ Angiotensin-converting enzyme inhibitors, 2 = Nonsteriodal anti- \\
inflamatory drugs, 3 = Cholesterol-lowering agents, 4 = Anti-thrombotic medication, 5 = Immunosuppressive agents, \\
6 = Proton pump inhibitors, 7 = Antidepressants.
\end{tabular}

\section{Discussion}

This study aimed (1) to develop an exercise testing method based on principles of exercise physiology to provoke cardiovascular responses and to evaluate aerobic capacity in post-stroke individuals with severe motor impairments, (2) to assess whether this new method would lead to acquisition of usable cardiovascular performance data based on international guidelines for exercise testing and prescription, (3) to evaluate the feasibility and safety of the procedure, and (4) to propose future research directions for exercise testing early after stroke.

\section{Constant load testing (CLT)}

Subjects tolerated all five stages of CLT. Time constants of oxygen uptake kinetics yielded reasonable values compared to previous studies following chronic stroke [23]. However, goodness of fit was not high in most of the analyses and the results of T1 and T2 differed widely. This might be due to the small step change employed. Oxygen uptake kinetics in the transition from 
rest to constant load exercise have been well described by implementing a moderate step change of $40-50 \% \dot{\mathrm{VO}}_{2}$ peak $[24,25]$. Our approach, calculating step response kinetics for both steps during CLT does not appear to be sensitive enough. We only displayed the step from rest to passive, but further approaches have to implement the step change by loading the subjects directly from rest to BWSmin. Furthermore, options to decrease BWS in a fast and safe way are required to ensure the subjects progressive load bearing activity to maintain their associated physiological gait pattern.

$\dot{\mathrm{VO}}_{2}$ during rest $(3.9 \pm 0.4 \mathrm{~mL} / \mathrm{min} / \mathrm{kg})$ yielded comparable values to a previous study using $30 \%$ BWS $(3.7 \pm 0.8 \mathrm{~mL} / \mathrm{min} / \mathrm{kg})$ in subjects after stroke [15]. Thus, we hypothesise that oxygen uptake is not much affected by BWS during standing. The steady-state increase in oxygen uptake from resting to the passive phase confirms that robotics-assisted walking requires some physical activity, as documented previously $[14,15]$. The recruitment of muscle mass during RATE might be much higher than in conventional procedures such as leg or arm cycle ergometry, even during low levels of load. This result is highly important for severely affected individuals who are not able to directly react to a target work rate mechanism.

Interestingly, the step from $80 \%$ BWS to $50 \%$ BWS was less distinctive than assumed, as reflected in most of the outcomes. This underlines the observation that the step increase was too low to consistently provoke a substantial cardiovascular response. However, conditions $80 \%$ and $50 \%$ BWS both yielded a substantially higher cardiovascular response compared to resting, which is an important finding for further research efforts regarding the evaluation of aerobic capacity after severe stroke.

\section{Incremental exercise testing (IET)}

The GF control strategy was not able to produce a substantial cardiovascular response during IET. Implementing GF control resulted in most cases to an abnormal gait pattern for GF values in the range $40-10 \%$, confirming a hypothesis of a previous study [26]. Subjects were overstrained on the affected leg at GF levels below 50\%, which led to an abnormal walking pattern due to motor impairment, weakness, and/or spasticity. Therefore, the termination criterion during all IETs was the inability to follow a physiological gait pattern. Although cardiovascular responses were present in some cases, GF control was not feasible and would need major adaptations. We hypothesise that subjects were more challenged by the coordinative task to successfully walk within the GF control, than by an increase in self-walking activity. A possible option would be to actuate the legs independently with different GF levels. And even better, appropriate assisted-as-needed approaches are in demand to optimally support individuals during their impaired gait cycle. 


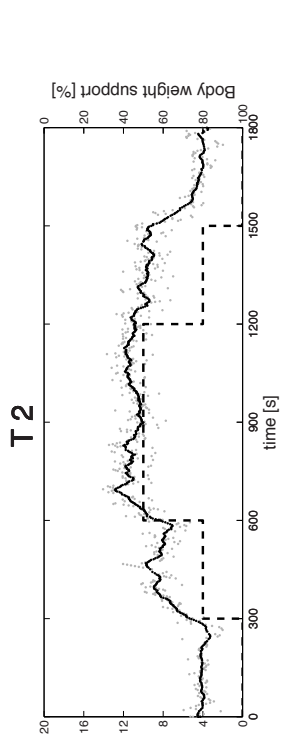

[6y/u!̣/um] ${ }^{2} \mathrm{O}$

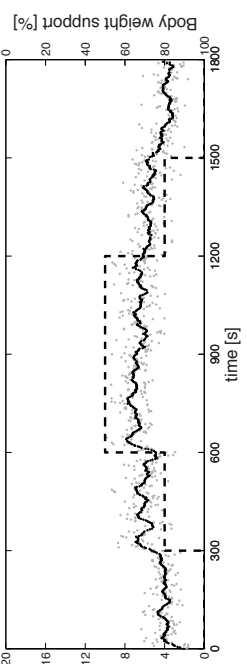

[6y/u!u/u/m] ${ }^{2} \mathrm{O} \wedge$
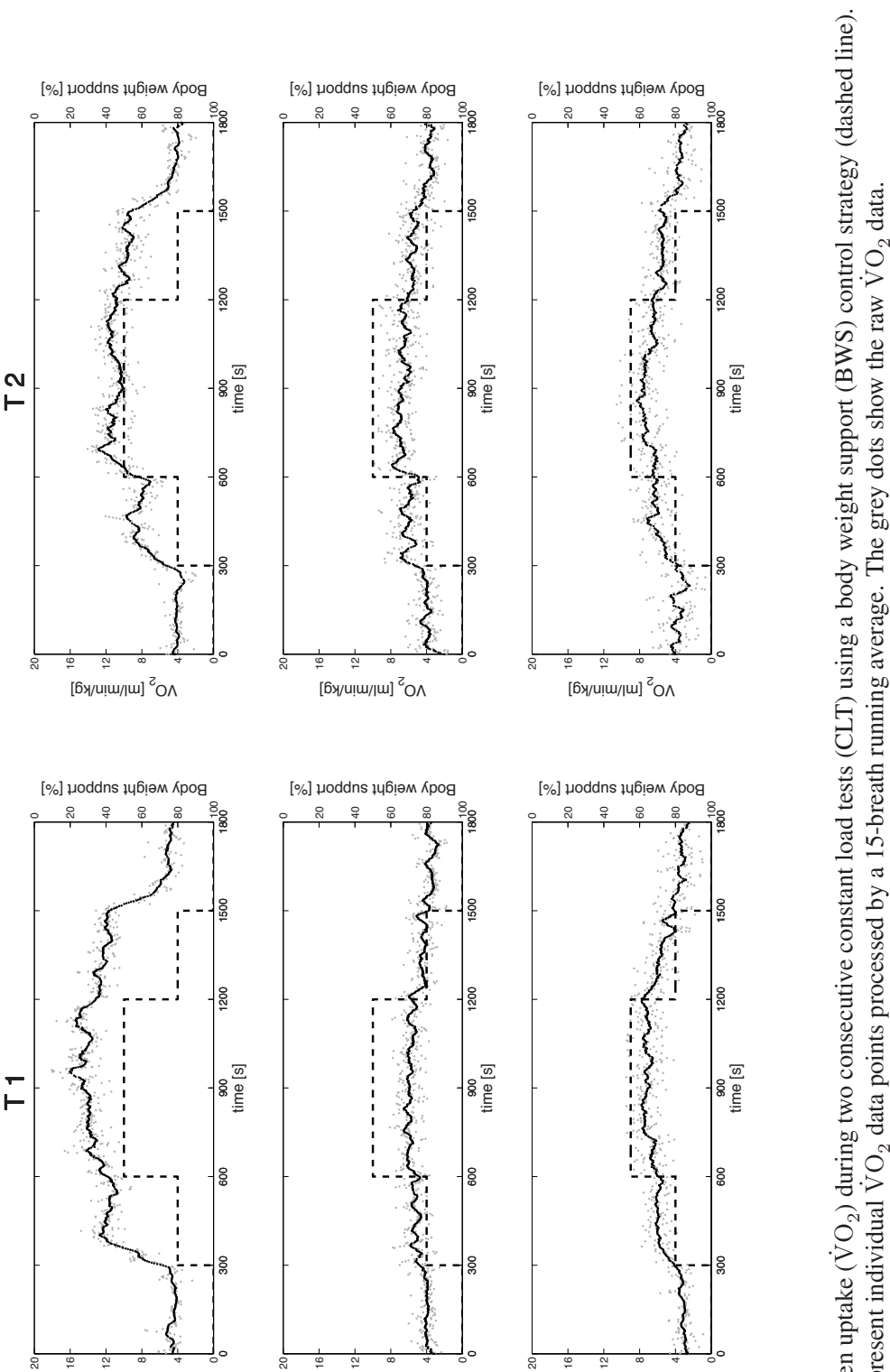

[6y/u!w/|u] ${ }^{\mathrm{Z} O \wedge}$

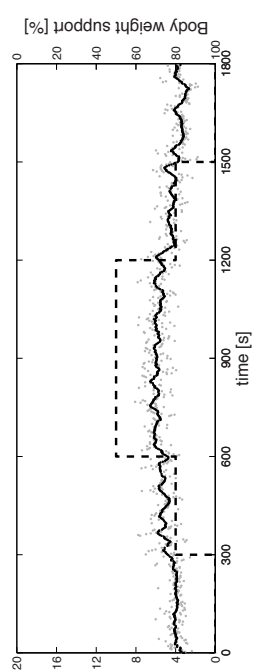

[6y/u!u/|u] ${ }^{2} \mathrm{O} /$

N
ปั
$\frac{6}{0}$
$\stackrel{5}{5}$

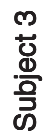

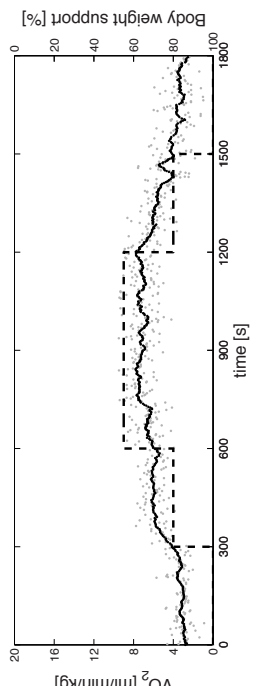

[6y/u!

$\overline{0}$
$\frac{0}{0}$
क

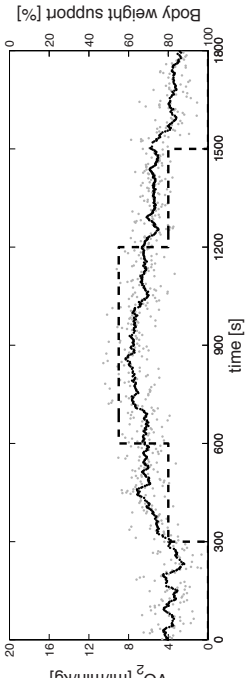

荬

일

क

i

홍

के

专㐫

त

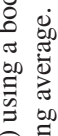

它声

के

宽

光

预

: ठ ठ

亲

总

응

哏

bo

恶.

กิ 즐

2

语.

营 专

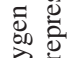

这苛

ô. :

लें 증

mั

尊 

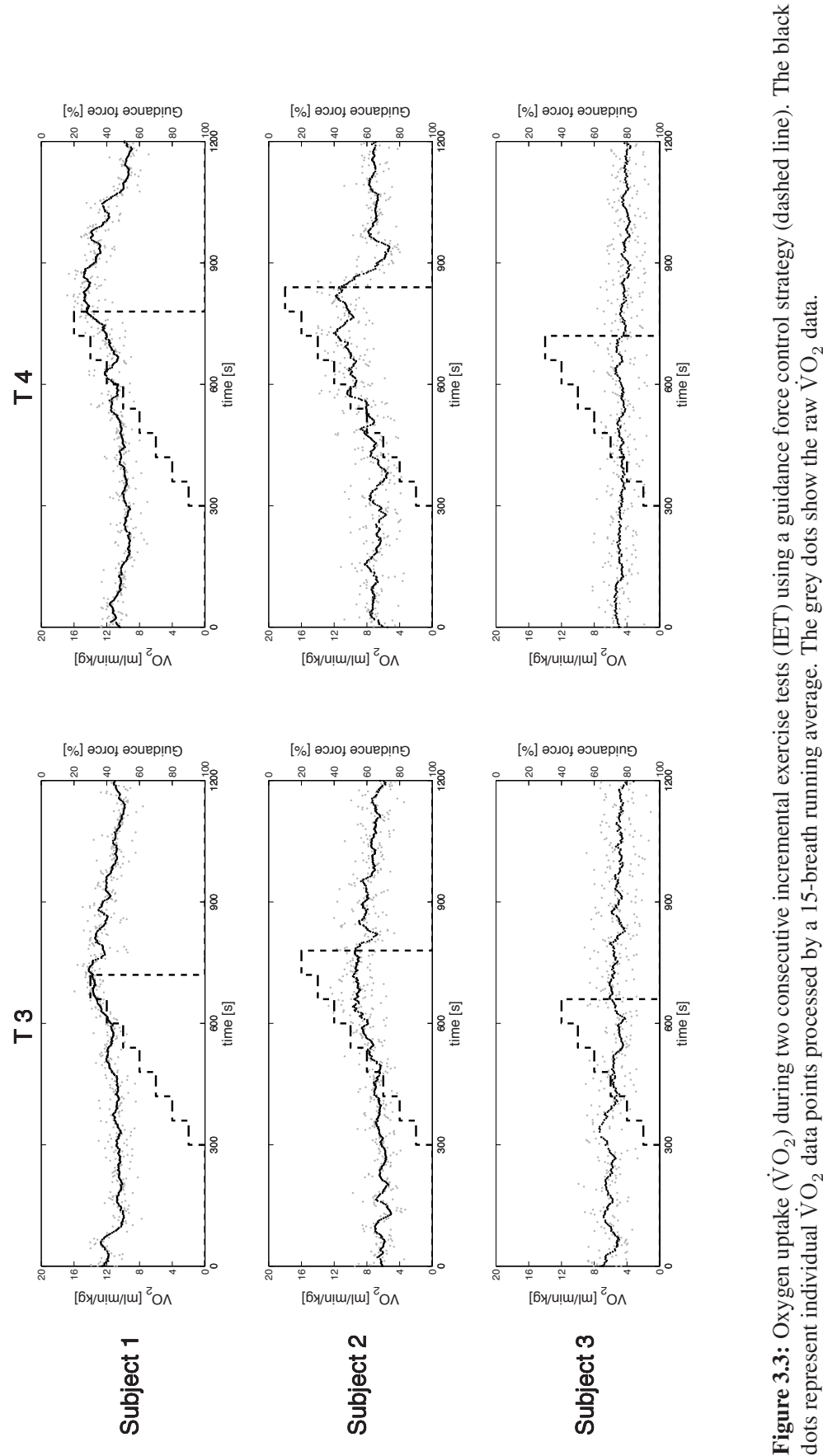


\section{Limitations}

The step changes implemented during CLT were too low to generate a significant response level of the cardiovascular system for detection of step response kinetics of $\dot{\mathrm{VO}}_{2}$. Second, it is in practice difficult to achieve a normal gait pattern with BWS in range $80-100 \%$. Therefore, we used $80 \%$ BWS during the passive phase, which affected the magnitude of the step in CLT. Third, we could

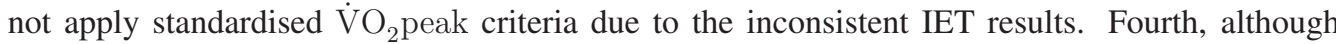
the subjects were similar in all parameters such as lesion, time post-stroke, and walking ability, subject 3 had a low cognitive status, which may have been disadvantageous for adaptation during GF control. Fifth, the study sample was small, but appropriate for a proof-of-concept study design.

\section{Conclusion}

The concept of exercise testing in individuals with severe motor impairments following stroke using a BWS control strategy for CLT is deemed feasible and safe. Our approach yielded reasonable results regarding cardiovascular performance parameters. Further research should focus on achieving an appropriate step magnitude for CLT, by decreasing BWS from 100\% to BWSmin as rapidly as possible. This may increase the accuracy of calculating step response kinetics of oxygen uptake. In addition, significant step response values might be used to prove the effectiveness of cardiovascular exercise interventions in severely impaired individuals. Furthermore, future studies may compare the steady-state improvements of predefined constant load levels to explore its association to peak exercise capacity.

IET based on GF control does not reliably provoke peak cardiovascular response due to uncoordinated walking patterns. Future research should design suitable active participation strategies to enforce peak performance in a safe and physiological way. Alternative rehabilitation robotics devices with stair climbing simulation might have the potential to substantially increase cardiovascular response in severely impaired individuals. 


\section{References}

[1] J. Hidler, D. Nichols, M. Pelliccio, K. Brady, D. D. Campbell, J. H. Kahn, and T. G. Hornby, "Multicenter randomized clinical trial evaluating the effectiveness of the lokomat in subacute stroke," Neurorehabilitation and Neural Repair, vol. 23, no. 1, pp. 5-13, 2009.

[2] T. G. Hornby, D. D. Campbell, J. H. Kahn, T. Demott, J. L. Moore, and H. R. Roth, "Enhanced gait-related improvements after therapist- versus robotic-assisted locomotor training in subjects with chronic stroke: a randomized controlled study," Stroke; a Journal of Cerebral Circulation, vol. 39, no. 6, pp. 1786-1792, 2008.

[3] B. Husemann, F. Müller, C. Krewer, S. Heller, and E. Koenig, "Effects of locomotion training with assistance of a robot-driven gait orthosis in hemiparetic patients after stroke: a randomized controlled pilot study," Stroke; a Journal of Cerebral Circulation, vol. 38, no. 2, pp. 349-354, 2007.

[4] A. Mayr, M. Kofler, E. Quirbach, H. Matzak, K. Fröhlich, and L. Saltuari, "Prospective, blinded, randomized crossover study of gait rehabilitation in stroke patients using the lokomat gait orthosis," Neurorehabilitation and Neural Repair, vol. 21, no. 4, pp. 307-314, 2007.

[5] J. Mehrholz, C. Werner, J. Kugler, and M. Pohl, "Electromechanical-assisted training for walking after stroke," Cochrane Database of Systematic Reviews (Online), no. 4, p. CD006185, 2007.

[6] K. P. Westlake and C. Patten, "Pilot study of lokomat versus manual-assisted treadmill training for locomotor recovery post-stroke," Journal of Neuroengineering and Rehabilitation, vol. 6, p. 18, 2009.

[7] I. Schwartz, A. Sajin, I. Fisher, M. Neeb, M. Shochina, M. Katz-Leurer, and Z. Meiner, "The effectiveness of locomotor therapy using robotic-assisted gait training in subacute stroke patients: a randomized controlled trial," PMR, vol. 1, no. 6, pp. 516-523, 2009.

[8] E. J. Roth, "Heart disease in patients with stroke: incidence, impact, and implications for rehabilitation. part 1: Classification and prevalence," Archives of Physical Medicine and Rehabilitation, vol. 74, no. 7, pp. 752-760, 1993.

[9] C. E. Hafer-Macko, A. S. Ryan, F. M. Ivey, and R. F. Macko, "Skeletal muscle changes after hemiparetic stroke and potential beneficial effects of exercise intervention strategies," Journal of Rehabilitation Research and Development, vol. 45, no. 2, pp. 261-272, 2008.

[10] K. Potempa, M. Lopez, L. T. Braun, J. P. Szidon, L. Fogg, and T. Tincknell, "Physiological outcomes of aerobic exercise training in hemiparetic stroke patients," Stroke, vol. 26, no. 1, pp. 101-105, 1995.

[11] O. Stoller, E. D. de Bruin, R. H. Knols, and K. J. Hunt, "Effects of cardiovascular exercise early after stroke: systematic review and meta-analysis," BMC Neurology, vol. 12, no. 1, p. 45, 2012.

[12] M. J. MacKay-Lyons and L. Makrides, "Cardiovascular stress during a contemporary stroke rehabilitation program: Is the intensity adequate to induce a training effect?," Archives of Physical Medicine and Rehabilitation, vol. 83, no. 10, pp. 1378-1383, 2002.

[13] H. S. Jorgensen, H. Nakayama, H. O. Raaschou, and T. S. Olsen, "Recovery of walking function in stroke patients: the copenhagen stroke study," Arch Phys Med Rehabil, vol. 76, no. 1, pp. 27-32, 1995.

[14] T. G. Hornby, D. H. Zemon, and D. Campbell, "Robotic-assisted, body-weight-supported treadmill training in individuals following motor incomplete spinal cord injury," Physical Therapy, vol. 85, no. 1, pp. 52-66, 2005.

[15] C. Krewer, F. Müller, B. Husemann, S. Heller, J. Quintern, and E. Koenig, "The influence of different lokomat walking conditions on the energy expenditure of hemiparetic patients and healthy subjects," Gait Posture, vol. 26, no. 3, pp. 372-377, 2007.

[16] L. P. Jack, M. Purcell, D. B. Allan, and K. J. Hunt, "Comparison of peak cardiopulmonary performance parameters during robotics-assisted treadmill exercise and arm crank ergometry in incomplete spinal cord injury," Technology and Health Care, vol. 18, no. 4-5, pp. 285-296, 2010. 
[17] K. J. Hunt, L. P. Jack, A. Pennycott, C. Perret, M. Baumberger, and T. H. Kakebeeke, "Control of work ratedriven exercise facilitates cardiopulmonary training and assessment during robot-assisted gait in incomplete spinal cord injury," Biomedical Signal Processing and Control, vol. 3, no. 1, pp. 19-28, 2008.

[18] O. Stoller, M. Schindelholz, L. Bichsel, C. Schuster, R. A. de Bie, E. D. de Bruin, and K. J. Hunt, "Feedbackcontrolled robotics-assisted treadmill exercise to assess and influence aerobic capacity early after stroke: a proofof-concept study," Disabil Rehabil Assist Technol, vol. 9, no. 4, pp. 271-278, 2014.

[19] M. Schindelholz, O. Stoller, and K. J. Hunt, "A software module for cardiovascular rehabilitation in roboticsassisted treadmill exercise,” Biomedical Signal Processing and Control, vol. 10, pp. 296-307, 2014.

[20] W. R. Thompson, N. F. Gordon, and L. S. Pescatello, American College of Sports Medicine. Guidelines for Exercise Testing and Prescription. Philadelphia: Lippincott Williams Wilkins, eight ed., 2010.

[21] H. Tanaka, K. D. Monahan, and D. R. Seals, "Age-predicted maximal heart rate revisited," Journal of the American College of Cardiology, vol. 37, no. 1, pp. 153-156, 2001.

[22] R. A. Robergs, D. Dwyer, and T. Astorino, "Recommendations for improved data processing from expired gas analysis indirect calorimetry," Sports Medicine, vol. 40, no. 2, pp. 95-111, 2010.

[23] P. J. Manns, C. R. Tomczak, A. Jelani, and R. G. Haennel, "Oxygen uptake kinetics: associations with ambulatory activity and physical functional performance in stroke survivors," J Rehabil Med, vol. 42, no. 3, pp. 259-264, 2010 .

[24] B. J. Whipp, "Domains of aerobic function and their limiting parameters," Physiology and Pathophysiology of Exercise Tolerance, pp. 83-89, 1996.

[25] F. Ozyener, H. B. Rossiter, S. A. Ward, and B. J. Whipp, "Influence of exercise intensify on the on- and offtransient kinetics of pulmonary oxygen uptake in humans," Journal of Physiology-London, vol. 533, no. 3, pp. 891-902, 2001.

[26] R. Riener, L. Lünenburger, S. Jezernik, M. Anderschitz, G. Colombo, and V. Dietz, "Patient-cooperative strategies for robot-aided treadmill training: first experimental results," IEEE Transactions on Neural Systems and Rehabilitation Engineering, vol. 13, no. 3, pp. 380-394, 2005. 


\section{Chapter 4}

\section{Feedback-controlled robotics-assisted treadmill exercise to assess and influence aerobic capacity early after stroke: a proof-of-concept study}

Disability and Rehabilitation: Assistive Technology 2014;9(4):271-278 doi: $10.3109 / 17483107.2013 .785038$

Oliver Stoller

Matthias Schindelholz

Lukas Bichsel

Corina Schuster

Rob A de Bie

Eling D de Bruin

Kenneth J Hunt 


\section{Abstract}

Purpose: The majority of post-stroke individuals suffer from low exercise capacity as a secondary reaction to immobility. The aim of this study was to prove the concept of feedback-controlled robotics-assisted treadmill exercise (RATE) to assess aerobic capacity and guide cardiovascular exercise in severely impaired individuals early after stroke.

Method: Subjects underwent constant load and incremental exercise testing using a human-inthe-loop feedback system within a robotics-assisted exoskeleton (Lokomat, Hocoma AG, CH). Inclusion criteria were: stroke onset $\leq 8$ weeks, stable medical condition, non-ambulatory status, moderate motor control of the lower limbs and appropriate cognitive function. Outcome measures included oxygen uptake kinetics, peak oxygen uptake ( $\dot{\mathrm{VO}}_{2}$ peak), gas exchange threshold (GET), peak heart rate (HRpeak), peak work rate (Ppeak) and accuracy of reaching target work rate $\left(\mathrm{RMSE}_{\mathrm{P}}\right)$.

Results: Three subjects (18-42 d post-stroke) were included. Oxygen uptake kinetics during constant load ranged from 42.0 to $60.2 \mathrm{~s}$. Incremental exercise testing showed: $\dot{\mathrm{V}} \mathrm{O}_{2}$ peak range 19.7$28.8 \mathrm{~mL} / \mathrm{min} / \mathrm{kg}$, GET range 11.6-12.7 mL/min/kg, and HRpeak range 115-161 beats/min. Ppeak range was 55.2-110.9 $\mathrm{W}$ and $\mathrm{RMSE}_{\mathrm{P}}$ range was 3.8-7.5 W.

Conclusion: The concept of feedback-controlled RATE for assessment of aerobic capacity and guidance of cardiovascular exercise is feasible. Further research is warranted to validate the method on a larger scale. 


\section{Background}

Stroke is one of the leading causes of long-term disability in western countries [1]. Rehabilitation strategies for individuals after stroke have focused primarily on restoring reduced motor control $[2,3]$. However, around $75 \%$ of individuals post-stroke exhibit cardiac disease $[4,5]$, and the majority have low endurance for exercise as secondary reaction to immobility $[6,7]$.

Previous work has shown that maximum oxygen uptake is reduced to $10-17 \mathrm{~mL} / \mathrm{min} / \mathrm{kg}$ within 0-30 d after stroke $[8,9,10]$ and does not rise over $20 \mathrm{~mL} / \mathrm{min} / \mathrm{kg}$ when individuals enter the chronic phase ( $>6$ month post-stroke) $[7,10,11,12]$. This early and persistent decline in aerobic capacity could delay or inhibit participation in a therapeutic exercise routine, complicate the rehabilitation process and the long-term post-stroke course of care, and limit the ability of the individual to perform functional activities independently [5, 13].

Recent meta-analyses have shown that aerobic exercise in the subacute phase after stroke is beneficial for improving aerobic capacity in people with mild to moderate motor impairment $[14,15]$. The feasibility and medical safety of exercise testing using leg cycle ergometry in this population has also been proven $[10,16,17]$. However, methods to assess aerobic capacity and guide cardiovascular exercise in severely impaired individuals after stroke are lacking. As a consequence, limited evidence is available about the impact of early cardiovascular exercise on mortality, motor recovery, independence in activities of daily living and quality of life in this population. Given the fact that most motor recovery occurs in the first 3 month after stroke [18], early cardiovascular exercise might have the potential to support and even accelerate the course of recovery by increasing an individual's vascular capacity.

The selection of appropriate modalities to assess and influence aerobic capacity in severely impaired individuals early after stroke is challenging due to associated medical conditions that may interfere with and/or complicate conventional procedures such as leg cycle ergometry or treadmill exercise. Moreover, around 50\% of post-stroke individuals may be non-ambulatory or even unable to walk a speed or distance necessary to achieve aerobic benefits [19] which, in turn, leads to inadequate training programmes and precludes exercise testing.

Considering the relatively short time frame of intensive rehabilitation after stroke, highly effective methods are needed to combine neuromuscular recovery training with cardiovascular exercise by using functional activities such as walking or stair climbing. The integration of roboticsassisted walking devices into cardiovascular rehabilitation has in this context potential to be of clinical relevance. The technology offers several advantages over conventional rehabilitation strategies such as controlled training and evaluation settings even for severely impaired individuals. 
Robotics-assisted treadmill exercise (RATE) enables the introduction of treadmill walking to individuals showing low levels of motor function and, thus, opens new perspectives for assessment of aerobic capacity and guidance of cardiovascular exercise. Initial work has shown that passive RATE provokes an increase in oxygen uptake [20,21]. Voluntary effort leads to similar oxygen uptake values as regular treadmill training [22, 23].

Considering the foregoing we developed novel assessment protocols to estimate key cardiovascular performance parameters during RATE [24, 25]. This incorporates a new biofeedback mechanism, which allows individuals to maximise their voluntary effort and associated cardiovascular stress. The underlying assumption driving this research was that feedback-controlled RATE might offer the possibility to evaluate aerobic capacity and guide cardiovascular rehabilitation in severely impaired individuals [26, 27] due to an increased recruitment of muscle mass during walking within the exoskeleton. A first study on individuals with spinal cord injury confirmed feasibility and validity of our approach [28].

New assessments and treatments usually have to go through a series of phases to test whether they are safe and effective before a larger scale study is to be considered [29]. The purpose of this study was, therefore, to evaluate the feasibility of the concept of using feedback-controlled RATE to assess aerobic capacity and guide cardiovascular exercise early after stroke. The study aimed to (1) develop an exercise testing and intervention method based on principles of exercise physiology and deliver it to severely impaired individuals post-stroke using a single case study design, (2) assess whether the concept would lead to acquisition of usable data sets, (3) evaluate the feasibility of the procedure and (4) to identify recommendations for future research directions regarding the assessment of aerobic capacity and guidance of cardiovascular exercise early after stroke.

\section{Methods}

\section{Subjects}

Three inpatients after stroke were recruited from a rehabilitation centre in the north-western part of Switzerland (Reha Rheinfelden) and screened by the responsible ward physician and a cardiologist according to the selection criteria. Inclusion criteria were: (1) clinical diagnosis of initial stroke (ischaemic or haemorrhagic), (2) $\leq 8$ weeks post stroke, (3) age $\geq 18$ years, (4) stable medical condition, (5) Mini Mental State Examination (MMSE) score of $\geq 24$, (6) Functional Ambulation Classification (FAC) of $\leq 3$ and (7) Chedoke-McMaster Stroke Assessment (CMSA) leg score of $\geq 2$ (where active voluntary movement is present without facilitation). Subjects were excluded if 
they had (1) cardiac contraindications for exercise testing according to the American College of Sports Medicine (ACSM) [30] and (2) contraindications for RATE according to guidelines from the manufacturer (Hocoma AG, Volketswil, Switzerland).

Recorded characteristics included sex, age, weight, height, diagnosis, body side affected, time post-stroke, additional diagnoses, medication and functional ability using the Extended Barthel Index (EBI). None of the subjects had received any significant physical exercise training prior to stroke. All subjects were informed about risks and benefits, and gave signed informed consent. The Ethics Review Committee of the Swiss Canton of Aargau approved the study (Reference No: 2011/017).

\section{Technical Implementation}

The Lokomat system (Hocoma AG, Volketswil, Switzerland) with an integrated treadmill (h/ p/ cosmos sports medical GmbH, Traunstein, Germany) and a motor-driven body weight support (BWS) system with real time feedback control for precise body weight unloading (Lokolift, Hocoma AG) was used. The exoskeleton provides active control at the hip and knee joints of both legs using DC motors. Real-time access to the joint forces and angular velocities was available via analogue signals from a custom interface unit (Lokomaster Output Box, Hocoma AG, Volketswil, Switzerland). The total mechanical work rate exerted on the exoskeleton by the subject was computed from the force, moment arm and velocity data at the four active joints. The active mechanical work rate $\left(\mathrm{P}_{\text {mech }}\right)$, applied by the subjects effort was estimated by subtracting the passive mechanical work rate (work rate necessary to move the subject passively within the exoskeleton) from the total mechanical work rate. A manual human-in-the-loop feedback system was implemented to control the subjects active work rate, programmed using LabVIEW software (Version 2009, National Instruments, Austin, TX) [31]. $\mathrm{P}_{\text {mech }}$ was smoothed by a first order IIR low-pass filter at $20 \mathrm{mHz}$ bandwidth and projected onto a screen at the front of the treadmill together with a target mechanical work rate $\left(\mathrm{P}_{\text {mech }}^{*}\right)$. The subject was instructed to vary the forces applied on the exoskeleton by volitional muscle activity and to keep the measured and visualised active work rate as close as possible to the target (Figure 4.1).

\section{Experimental Protocol}

The first session focused on adjusting the Lokomat system to provide a physiological gait pattern and to ensure that the subjects could walk comfortably in the exoskeleton. The second session allowed familiarisation with the facemask for breath-by-breath monitoring, and the implementation 


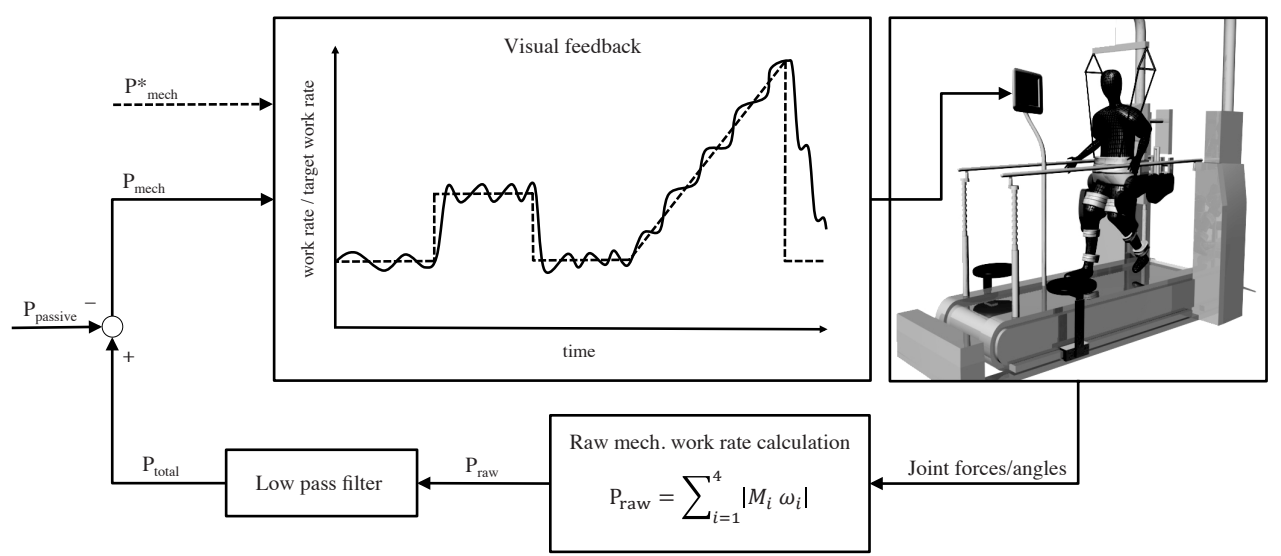

Figure 4.1: Human-in-the-loop work rate control during robotics-assisted treadmill exercise (RATE). The solid line represents the mechanical work rate $\left(\mathrm{P}_{\text {mech }}\right)$ produced by the subject, whereas the dashed line represents the target work rate $\left(\mathrm{P}_{\text {mech }}^{*}\right)$. The passive mechanical work rate $\left(\mathrm{P}_{\text {passive }}\right)$ is evaluated before every session and subtracted from $\mathrm{P}_{\text {mech }}$. Legend: $\mathrm{P}_{\text {raw }}=$ raw mechanical work rate, $M_{i}=$ moments of force, $\omega_{i}=$ angular velocity, $\mathrm{P}_{\text {total }}=$ total mechanical work rate.

of maximal voluntary contractions during walking (MVC-W) in the exoskeleton to determine the individual work rate maximum (Pmax). Subjects were asked to increase their maximal voluntary effort during RATE within 10s. MVC-W was repeated three times, and the mean Pmax values were used for further calculations.

Each subject underwent constant load testing (CLT) and incremental exercise testing (IET) (Figure 4.2). The CLT consisted of 15 min constant-intensity exercise ( $\left.\mathrm{P}_{\text {mech }}^{*}=40 \% \mathrm{Pmax}\right)$. Subjects were instructed to: (1) stand on the treadmill attached to the exoskeleton with $50 \%$ BWS and no treadmill motion for 5 min (recorded rest), (2) walk passively by letting the exoskeleton move their legs for $5 \mathrm{~min}$ (passive phase), (3) to actively contribute to the walking by pushing against the exoskeleton to reach the individual $\mathrm{P}_{\text {mech }}^{*}$ for 15 min (active phase) and (4) walk passively by letting the exoskeleton move their legs for $5 \mathrm{~min}\left(2^{\text {nd }}\right.$ passive phase). The IET consisted of continuous ramp exercise where the slope was designed to reach the individual Pmax at $15 \mathrm{~min}$. Subjects were instructed to: (1) stand on the treadmill attached to the exoskeleton with 50\% BWS and no treadmill motion for $5 \mathrm{~min}$ (recorded rest), (2) walk passively by letting the exoskeleton move their legs for $5 \mathrm{~min}$ (passive phase), (3) to actively contribute to the walking by pushing against the exoskeleton to reach the individual $\mathrm{P}_{\text {mech }}^{*}$ until volitional exhaustion (active phase) and (4) walk passively by letting the exoskeleton move their legs for $5 \min \left(2^{\text {nd }}\right.$ passive phase).

Termination criteria for all tests were: (a) abnormal blood pressure responses, i.e. hypertensive 
(systolic BP $\geq 210 \mathrm{~mm} \mathrm{Hg}$ or diastolic $\mathrm{BP} \geq 115 \mathrm{~mm} \mathrm{Hg}$ ) when exercising at high work rate, or hypotensive responses (decrease in $\mathrm{BP} \geq 10 \mathrm{~mm} \mathrm{Hg}$ ) despite an increase in work rate, (b) individual $\mathrm{P}_{\text {mech }}$ below $\mathrm{P}_{\text {mech }}^{*}$ for $\geq 30 \mathrm{~s}$, (c) age-predicted heart rate maximum [32], whereas the formula was adjusted accordingly to $70 \%$ for subjects on b-blocker medications [33] and (d) pain or discomfort in the chest.

The subjects were scheduled for the protocols within a 2-week time period. The first week was reserved for two CLTs with $48 \mathrm{~h}$ rest between the experiments and the second week contained two IETs with $48 \mathrm{~h}$ rest between tests. The aim of this sequence was to account for possible adverse events or unwanted effects during the experimental procedure to obtain at least one successful test per protocol.

Treadmill speed was fixed at $2 \mathrm{~km} / \mathrm{h}$ and BWS was $50 \%$ of individual body mass for all sessions. Blood pressure was measured prior to all tests in a sitting position and every 3 min while performing the test using a sphygmomanometer (HEM-907, Omron Corporation, Kyoto, Japan). There was close adherence to established models for exercise testing according to the ACSM [30].

\section{Safety Arrangements}

There is an overall risk for exercise after stroke regarding a recurrent cerebral or cardiac event. Furthermore, the subject is vertically strapped to the exoskeleton, which complicates emergency procedures. Several risk management arrangements were implemented: (1) clearly defined eligibility criteria, (2) continuous blood pressure monitoring (cut off: systolic $\geq 210$, diastolic $\geq 115$, hypotensive responses $\geq 10 \mathrm{~mm} \mathrm{Hg}$ ), (3) presence of resuscitation-trained assistants, (4) opportunity to call the medical resuscitation team in the clinic and (5) trained research personnel to be able to release the subject within $60 \mathrm{~s}$ from the exoskeleton.

\section{Outcomes}

Aerobic capacity was assessed by breath-by-breath spirometry (MetaMax 3B, Cortex Biophysik, Leipzig, Germany). Heart rate was measured continually and recorded by a heart rate belt (T31, Polar Electro, Kempele, Finland) and a receiver board (HRMI, Sparkfun, Boulder, CO). $\mathrm{P}_{\text {mech }}$ was calculated by the force and angular signals, which were available in real time from the custom interface unit. For CLT, outcome measures were time constants $(\tau)$ of oxygen uptake kinetics, steady-state increase in oxygen uptake following a step $\left(\Delta \dot{\mathrm{V}} \mathrm{O}_{2}\right)$, oxygen cost of work $\left(\Delta \dot{\mathrm{VO}}_{2} / \Delta \mathrm{P}\right)$, and accuracy of reaching target work rate $\left(\mathrm{RMSE}_{\mathrm{P}}\right)$. IET focused on peak performance parameters such as peak oxygen uptake ( $\dot{\mathrm{VO}}_{2}$ peak), gas exchange threshold (GET), peak 
respiratory exchange ratio (RERpeak), peak heart rate (HRpeak), peak work rate (Ppeak) and accuracy of reaching target work rate $\left(\mathrm{RMSE}_{\mathrm{P}}\right)$.

The feasibility of the test procedures was rated using the following outcomes: (i) feasible as is, (ii) feasible with modifications, (iii) not feasible [29]. We were interested in safety, compliance, cardiovascular responses to the exercise, continuous data processing and successful clinical implementation.

\section{CLT}

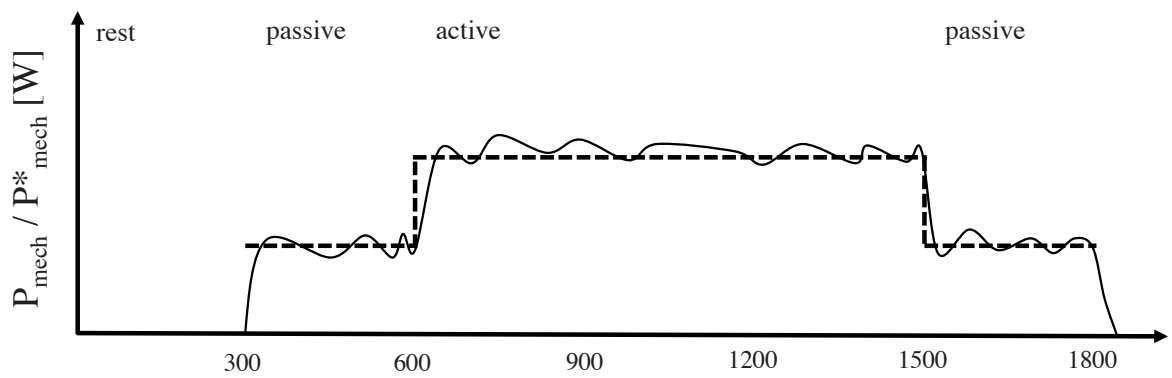

\section{IET}

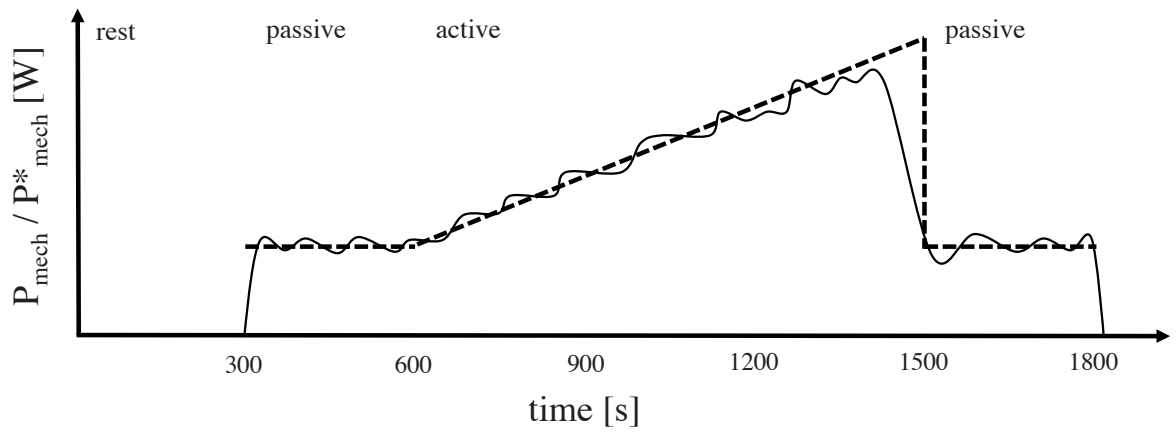

Figure 4.2: Schematic representation of constant load testing (CLT) and the incremental exercise testing (IET). The solid line represents the active work rate $\left(\mathrm{P}_{\text {mech }}\right)$ produced by the subject, whereas the dashed line represents the target work rate $\left(\mathrm{P}_{\text {mech }}^{*}\right)$. 


\section{Analysis}

Descriptive analyses were performed on all variables. To ensure clinical validity regarding the interpretation of the results, only the first complete CLT/IET was considered for analysis. Raw breath-by-breath data were processed using a 15-breaths running average, aligned to the time of the central breath [34]. For CLT, the time constant $(\tau)$ of oxygen uptake kinetics is defined as the time taken to reach $63 \%$ of the steady state $\mathrm{VO}_{2}$ following a step change in work rate during moderate exercise below the anaerobic threshold [35]. $\tau$ values were calculated by excluding the first $20 \mathrm{~s}$ and using a non-linear least-squares algorithm to fit the data as described in the following mono-exponential equation: $\dot{\mathrm{V}}_{2}(\mathrm{t})=\dot{\mathrm{V}}_{2}(\mathrm{~b})+\dot{\mathrm{V}} \mathrm{O}_{2}\left(1-\mathrm{e}^{-(\mathrm{t}-\mathrm{Td}) / \tau}\right), \mathrm{t}<0$ with $\dot{\mathrm{VO}}_{2}(\mathrm{~b})=\dot{\mathrm{V}} \mathrm{O}_{2}$ at baseline, $\Delta \dot{\mathrm{V}} \mathrm{O}_{2}=$ step increase in oxygen uptake, $\mathrm{Td}=$ time delay of $20 \mathrm{~s}$, and $\tau=$ time constant. To calculate the step increase in oxygen uptake $\left(\Delta \dot{\mathrm{V}} \mathrm{O}_{2}\right)$, steady state $\dot{\mathrm{V}} \mathrm{O}_{2}$ was defined as the mean value of minutes 3-5 during the constant load over time. The accuracy of reaching the target work rate was expressed by the root mean square error $(\mathrm{RMSE})$ between work rate $\left(\mathrm{P}_{\text {mech }}\right)$ and target work rate $\left(\mathrm{P}_{\text {mech }}^{*}\right)$. $\dot{\mathrm{V}} \mathrm{O}_{2}$ peak was defined as the mean $\dot{\mathrm{VO}}_{2}$ in the last $20 \mathrm{~s}$ during incremental exercise, whereas HRpeak was the maximal heart rate reached during IET. The gas exchange threshold (GET) represents the oxygen uptake value at the point at which carbon dioxide output begins to increase more rapidly in proportion to oxygen uptake. This was estimated using the v-slope method and standard gas exchange criteria [36]. All analyses and displays were performed using MATLAB (Version R2010a, MathWorks, Natick, MA).

\section{Results}

Subjects characteristics are displayed in Table 4.1. The preliminary evaluation of Pmax during MVC-W ranged from 58.1 to $130.7 \mathrm{~W}$. For CLT, time constants $(\tau)$ of oxygen uptake kinetics yielded values in the range of 42.0-60.2 s (Table 4.2). The results represented a moderate increase in steady-state oxygen uptake $\left(\Delta \dot{\mathrm{VO}}_{2}\right)$ from resting to passive walking in all subjects, and a substantial oxygen uptake increase from passive to active RATE $\left(\Delta \dot{\mathrm{VO}} \mathrm{O}_{2}=9.3 \mathrm{~mL} / \mathrm{min} / \mathrm{kg}\right)$ in subject 3 (Figure 4.3). RMSE $E_{P}$ values for reaching target work rate were low. However, subject 3 provoked an abrupt safety stop after $820 \mathrm{~s}$ that substantially increased $\mathrm{RMSE}_{\mathrm{P}}$, caused by temporary dis-coordination of the walking pattern within the exoskeleton.

For IET, peak aerobic performance yielded $\dot{\mathrm{V}} \mathrm{O}_{2}$ peak values of $19.7,19.8$, and $28.8 \mathrm{~mL} / \mathrm{min} / \mathrm{kg}$ respectively, whereby the time to $\dot{\mathrm{V}}{ }_{2}$ peak differed strongly between subjects (Figure 4.3). GET estimation showed similar values in all of the 3 subjects. RERpeak above 1.0 was reached in 2 subjects and percentage HRpeak to age predicted heart rate maximum was $69.9 \%, 77.4 \%$, and 
93.5\% respectively. Attention should be paid to the association between Pmax and Ppeak during IET, because differences between subjects in reaching Pmax were high (95.1\%, 107.5\%, 59.6\%). Mean $\mathrm{RMSE}_{\mathrm{P}}$ was below $10 \mathrm{~W}$, whereas termination of the incremental phase was due to individual work rate below target work rate for $30 \mathrm{~s}$ during all IET protocols. None of the subjects had hypertensive/hypotensive responses or pain/discomfort in the chest, and there were no adverse effects during any tests.

All subjects completed the tests without any safety issues, resulting in complete compliance with the test protocol. Given the very extensive instrumentation to perform the protocol, data processing rate and clinical implementation were completely successful. A trained therapist was responsible for the RATE preparations; it took about 10 min to prepare the subject for the experimental setup. A research assistant conducted the protocol and monitored the outcome measures. Each protocol was completed in a 1-h therapy setting during usual care. In view of these results, the overall feasibility of the concept was set to: (ii) feasible with modifications.

Table 4.1: Subject characteristics

$\begin{array}{llll} & \text { Subject } 1 & \text { Subject 2 } & \text { Subject 3 } \\ \text { Sex } & \text { Male } & \text { Male } & \text { Male } \\ \text { Age }(\mathrm{yr}) & 63 & 47 & 51 \\ \text { Body mass }(\mathrm{kg}) & 83 & 94 & 85 \\ \text { Body height }(\mathrm{cm}) & 180 & 173 & 176 \\ \text { BMI }\left(\mathrm{kg} / \mathrm{m}^{2}\right) & 25.6 & 31.4 & 27.4 \\ \text { Diagnosis } & \text { Ischemic stroke } & \text { Ischemic stroke } & \text { Ischemic stroke } \\ \text { Affected body side } & \text { Right } & \text { Right } & \text { Left } \\ \text { Days post event }(\mathrm{d}) & 42 & 18 & 19 \\ \text { Medication } & 1,2,3,4 & 1,3,4,6,8 & 1,3,4 \\ \text { MMSE }(0 / 30) & 30 & 30 & 30 \\ \text { FAC }(0 / 5) & 2 & 2 & 3 \\ \text { CMSA leg score }(1-7) & 5 & 5 & 5 \\ \text { EBI at clinical admission }(0 / 64) & 55 & 57 & 63 \\ \text { EBI at first session }(0 / 64) & 56 & 57 & 63 \\ \text { EBI at clinical discharge }(0 / 64) & 56 & 64 & 63 \\ \end{array}$

Abbreviations: $\mathrm{BMI}=$ Body Mass Index, MMSE = Mini Mental State Examination, FAC = Functional Ambulation Classification, CMSA = Chedoke McMaster Stroke Assessment, EBI = Extended Barthel Index, $1=$ Angiotensinconverting enzyme inhibitors, $2=$ Nonsteriodal anti-inflamatory drugs, 3 = Cholesterol-lowering agents, $4=$ Antithrombotic medication, $5=$ Immunosuppressive agents, $6=$ Proton pump inhibitors, $7=$ Antidepressants, $8=$ Antibiotics 

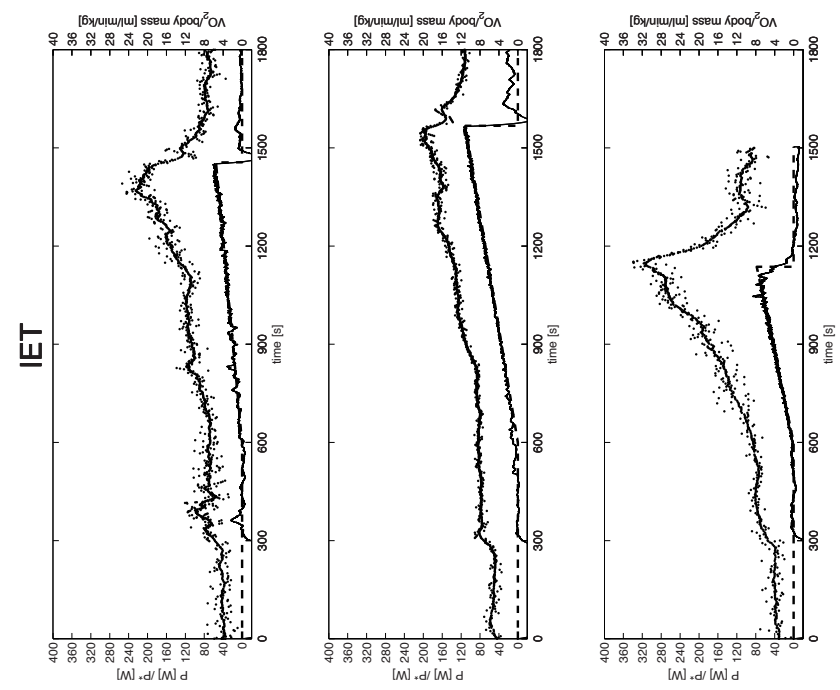

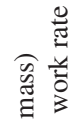

帘莣.

잉

氞总

氧焉

产递要

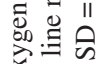

을

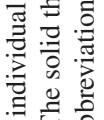

焉

矛 己ें

苛

苛离选

总会苛

击

记

武交导
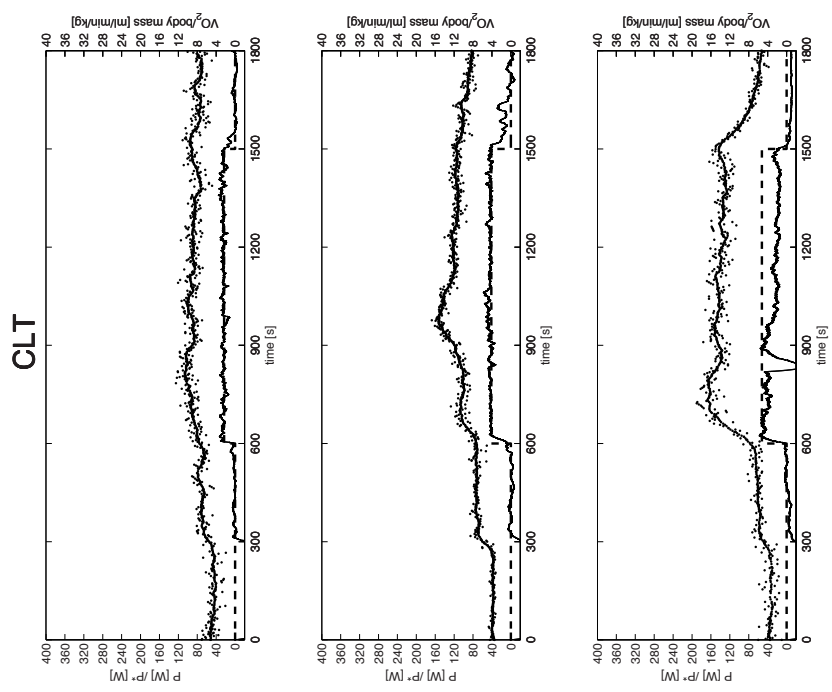

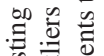

进表

政

矛

离.

局

อั ญ

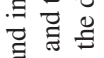

它范苛

bo bo

寻告苍

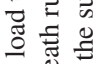

氙㝴

密尚豆

ป

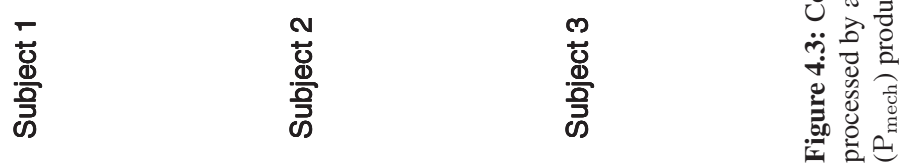


Table 4.2: Exercise testing results using feedback-controlled RATE

\begin{tabular}{|c|c|c|c|}
\hline & Subject 1 & Subject 2 & Subject 3 \\
\hline Pmax during MVC-W [W] & 58.1 & 103.16 & 130.69 \\
\hline \multicolumn{4}{|l|}{ CLT } \\
\hline Oxygen uptake kinetics $(\tau)[\mathrm{s}]$ & 60.19 & 42.31 & 42.04 \\
\hline $\mathrm{P}_{\text {mech }}^{*}$ during active phase $(40 \% \mathrm{MVC}-\mathrm{W})[\mathrm{W}]$ & 23.24 & 41.26 & 52.28 \\
\hline$\Delta \dot{\mathrm{V} O} \mathrm{O}_{2}$ from rest to passive $[\mathrm{mL} / \mathrm{min} / \mathrm{kg}]$ & 2.43 & 3.39 & 2.82 \\
\hline$\Delta \dot{\mathrm{VO}} \mathrm{O}_{2}$ from passive to active $[\mathrm{mL} / \mathrm{min} / \mathrm{kg}]$ & 3.02 & 3.04 & 9.26 \\
\hline$\dot{\mathrm{V}} \mathrm{O}_{2}$ cost of work $[\mathrm{mL} / \mathrm{min} / \mathrm{W}]$ & 7.59 & 10.63 & 13.08 \\
\hline Accuracy of target work rate $\left(\mathrm{RMSE}_{\mathrm{P}}\right)[\mathrm{W}]$ & 4.06 & 5.70 & 30.02 \\
\hline \multicolumn{4}{|l|}{ IET } \\
\hline$\dot{\mathrm{V}} \mathrm{O}_{2}$ peak $[\mathrm{mL} / \mathrm{min}]$ & 1636 & 1857 & 2449 \\
\hline$\dot{\mathrm{V}} \mathrm{O}_{2}$ peak/body mass $[\mathrm{mL} / \mathrm{min} / \mathrm{kg}]$ & 19.71 & 19.76 & 28.81 \\
\hline Time from ramp onset to $\dot{\mathrm{V}} \mathrm{O}_{2}$ peak $[\mathrm{s}]$ & 846 & 958 & 527 \\
\hline $\mathrm{GET}[\mathrm{mL} / \mathrm{min}]$ & 1052 & 1088 & 983 \\
\hline GET/body mass $[\mathrm{mL} / \mathrm{min} / \mathrm{kg}]$ & 12.67 & 11.58 & 11.56 \\
\hline RERpeak & 1.11 & 0.95 & 1.14 \\
\hline HRpeak [beats/min] & 115 & 136 & 161 \\
\hline HRpeak of predicted heart rate max [\%] & 69.86 & 77.38 & 93.50 \\
\hline Ppeak [W] & 55.24 & 110.89 & 77.95 \\
\hline Ppeak of Pmax [\%] & 95.08 & 107.49 & 59.64 \\
\hline Accuracy of target work rate $\left(\mathrm{RMSE}_{\mathrm{P}}\right)[\mathrm{W}]$ & 4.06 & 5.70 & 30.02 \\
\hline
\end{tabular}

Abbreviations: MVC-W = Maximal voluntary contraction during walking, RMSE = Root mean square error

\section{Discussion}

The aim of this proof-of-concept study was to (1) develop an exercise testing and intervention method based on principles of exercise physiology and deliver it to severely impaired individuals post-stroke using a single case study design, (2) assess whether the concept would lead to acquisition of usable data sets, (3) evaluate the feasibility of the procedure and (4) to identify recommendations for future research directions regarding the assessment of aerobic capacity and guidance of cardiovascular exercise early after stroke.

We demonstrated a novel cardiovascular exercise testing and intervention method for individuals with subacute stroke showing low levels of motor function. All subjects tolerated CLT and 
IET using feedback-controlled RATE. For CLT, our findings yield time constant values comparable to reference data in an earlier study evaluating oxygen uptake kinetics after stroke [37]. However, comparisons between studies are difficult because of non-standardised evaluation methods for calculation of the time constants of oxygen uptake kinetics. These values may nevertheless have importance for within-study comparisons as a measure of the subjects ability to adapt to a change in exercise work rate. Further trials should therefore optimise and standardise the calculation procedure. The time constants for recovery from a certain exercise work rate may give additional information regarding the oxygen uptake kinetics during RATE.

The steady-state increase in oxygen uptake values during the passive phase confirms the observation that robotics-assisted walking is not actually passive, as shown in previous studies $[20,22,23]$. We hypothesise that the recruitment of muscle mass during RATE is much higher than in conventional procedures such as leg or arm cycle ergometry, even during low levels of load. This response might be interesting for severely impaired individuals who are not able to voluntarily react within the work rate feedback-guidance mechanism of the approach presented here.

Peak performance results clearly confirmed the feasibility of the test concept and the technical feasibility of the method. Previous studies described $\dot{\mathrm{VO}}_{2}$ peak values of $10.7 \pm 0.38$ [10], $11.8 \pm 0.8$ [16], $11.4 \pm 3.7 \mathrm{~mL} / \mathrm{min} / \mathrm{kg}$ [17] for post-stroke individuals using leg cycle ergometry, and $13.3 \pm 2.7 \mathrm{~mL} / \mathrm{min} / \mathrm{kg}$ using treadmill exercise [11]. Our findings showed similar or higher $\dot{\mathrm{V}} \mathrm{O}_{2}$ peak values using feedback-controlled RATE. GET values were also similar to earlier studies which reported the values of $9.6 \pm 0.4$ [10], $8.4 \pm 1.8$ [16] and $8.6 \pm 1.7 \mathrm{~mL} / \mathrm{min} / \mathrm{kg}$ [17]. Betweenstudy comparisons of indirect anaerobic threshold calculations should be interpreted with caution, because comparisons of evaluation methods have shown large differences large differences $[36,38,39]$.

RERpeak values of $\geq 1.0$ during IET were only reached in subject 1 and 3 . This result suggests that $\mathrm{P}_{\text {mech }}^{*}$ probably demanded too little from subject 2 to force peak aerobic performance within the given time frame. It is recommended to reach peak cardiovascular performance between 480 and 720 s [30]. However, we defined Pmax at $90 \mathrm{~s}$ to give the subjects additional time to adjust to the situation, as it was a completely new approach. Based on these results, the assessment of Pmax using initial MVC-W requires further consideration. Our approach evaluated Pmax only during a single period at a very early stage of RATE that might have not led to a suitable estimation of $\mathrm{P}_{\text {mech }}^{*}$. This assumption is supported by the abrupt safety stop after $820 \mathrm{~s}$ during CLT of subject 3 due to a poor walking pattern within the exoskeleton and the fact that $\mathrm{P}_{\text {mech }}^{*}$ was not reached after recommencement of RATE. MVC-W might be useful to estimate Pmax, but it should be assessed 
at the start of each protocol individually. We hypothesise that subjects might increase Pmax by increasing motor coordination and efficiency during RATE, and that being in good or bad fettle on the day of testing might also influence the MVC-W protocol. In a similar way to established protocols using leg cycle ergometry and treadmill exercise, the challenge of estimating a subjects Pmax to calculate optimal baseline values remains. Furthermore, there is a lack of reference data regarding $\mathrm{P}_{\text {mech }}$ during $\mathrm{RATE}$, and these values are difficult to interpret because of the multifactorial interference that occurs during human-machine interaction, e.g. attachment of the body to the exoskeleton, holding of the handrails or the defined BWS may lead to disturbances. Further studies must evaluate the variance of Pmax for individuals showing large differences in motor capabilities to recommend optimal $\mathrm{P}_{\text {mech }}^{*}$ values by taking individuals impairment level into account. One opportunity might be a classification based on standardised motor recovery scores such as the Chedoke-McMaster Stroke Assessment or the Fugl-Meyer Assessment. As in established protocols, recommendations for $\mathrm{P}_{\text {mech }}^{*}$ regarding a certain start level and an incremental step increase over time are in demand.

The current work demonstrated the accuracy of reaching target work rate during feedbackcontrolled RATE. Subjects were able to follow $\mathrm{P}_{\text {mech }}^{*}$ during the entire testing protocol. Almost all $\mathrm{RMSE}_{\mathrm{P}}$ values were $\leq 10 \mathrm{~W}$. The major deviation of subject $3(30.0 \mathrm{~W})$, caused by a safety stop during CLT due to dis-coordination of the walking pattern, can be seen as a normal occurrence during RATE based on the challenges of the human-machine interaction mentioned above. These results confirm the feasibility of guiding cardiovascular exercise using a feedback-control structure within a robotics-assisted treadmill.

We did not focused too much on heart rate, as these measures are highly affected by day-byday variability, medication, and has been shown to be not accurate for individual estimations [40]. However, HRpeak values were high during IET and might have represented the course of effort during exercise testing. This item should, therefore, be closely monitored when data for a group of subjects are to be collected in the future.

The overall feasibility of the concept seems to be proven albeit with the need of some protocol modification. As discussed previously, the estimation of individual target work rate $\left(\mathrm{P}_{\text {mech }}^{*}\right)$ needs further consideration. Furthermore, Pmax values have to be set between 480 and $720 \mathrm{~s}$ to optimally demand peak performance. However, the concept has shown preliminary safety, overall compliance, successful data processing and clinical implementation, which is an important finding of this proof-of-concept approach.

The study has some limitations. First, for this new approach of estimating peak performance parameters using RATE, we did not employ termination criteria such as plateaus in $\dot{\mathrm{VO}}_{2}$ and RER 
values, as they were reported and used in previous studies $[10,11,16]$. This might have affected the estimation of aerobic capacity. Second, the present study protocol did not include ECG monitoring due to practicability, which influenced the eligibility assessment by excluding individuals with cardiac risk factors for exercise testing. Third, the sample size of this study was small, however, is justified based on a proof-of-concept methodology to evaluate the technical and clinical feasibility of a new method. The findings of these three single cases warrant the performance of a small group study.

In summary, the feasibility of the concept for assessment of aerobic capacity and guidance of cardiovascular exercise in non-ambulatory individuals post-stroke using feedback-controlled RATE has been established. Oxygen uptake kinetics may have advantages for the evaluation of aerobic capacity in severely disabled individuals with cardiac risk factors for exercise testing. We observed substantial peak aerobic performance values, but the evaluation of individual Pmax for the definition of optimal slope values for IET and the interpretation of $\mathrm{P}_{\text {mech }}$ remains challenging due to the multifactorial interference during the human-machine interaction. It is a future challenge to investigate true work rate during activities within an exoskeleton (e.g. robotics-assisted walking, -stair raising, -grasping). Safety and compliance are critical features of this new approach and we applied strict exclusion criteria for this small cohort, particularly as it was a proof-of-concept study. It is hoped that the criteria can be relaxed in time as experience is gained and the protocol may be extended to more severely impaired individuals.

Further research should focus on validity and reliability of exercise testing and on the feasibility of cardiovascular exercise prescription using feedback-controlled RATE. This new concept might have the potential to accurately evaluate aerobic capacity in severely impaired individuals. Moreover, the feedback-control structure is able to guide exercise intensity during RATE and could be used to implement early cardiovascular exercise. Prolonged constant load exercise on individual levels might be used to introduce regular therapeutic exercise in this population. Further investigations should give attention to the estimation of individual Pmax values while walking within an exoskeleton to define optimal target work rates. There could be a focus on the control of external influences during human-machine interaction (fixtures, active holding of the handrails, BWS settings, etc.). However, it will be necessary to define appropriate exercise protocols for individuals showing varying levels of motor impairment at different stages of recovery to effectively assess true aerobic capacity and guide cardiovascular exercise. 


\section{Conclusion}

The feasibility of the concept for assessment of aerobic capacity and guidance of cardiovascular exercise in severely impaired individuals post-stroke using feedback-controlled RATE seems given. This study yielded reasonable values for cardiovascular performance parameters following standardised exercise protocols in 3 individuals early after stroke. Further research is warranted to validate the method on a larger scale.

\section{References}

[1] G. A. Donnan, M. Fisher, M. Macleod, and S. M. Davis, "Stroke," Lancet, vol. 371, no. 9624, pp. 1612-1623, 2008.

[2] D. Wade, "Rehabilitation therapy after stroke," The Lancet, vol. 354, no. 9174, pp. 176-177, 1999.

[3] P. Langhorne, F. Coupar, and A. Pollock, "Motor recovery after stroke: a systematic review," Lancet Neurology, vol. 8, no. 8, pp. 741-754, 2009.

[4] E. J. Roth, "Heart disease in patients with stroke: incidence, impact, and implications for rehabilitation. part 1: Classification and prevalence," Archives of Physical Medicine and Rehabilitation, vol. 74, no. 7, pp. 752-760, 1993.

[5] E. J. Roth, "Heart disease in patients with stroke. part 2: Impact and implications for rehabilitation," Archives of Physical Medicine and Rehabilitation, vol. 75, no. 1, pp. 94-101, 1994.

[6] C. E. Hafer-Macko, A. S. Ryan, F. M. Ivey, and R. F. Macko, "Skeletal muscle changes after hemiparetic stroke and potential beneficial effects of exercise intervention strategies," Journal of Rehabilitation Research and Development, vol. 45, no. 2, pp. 261-272, 2008.

[7] K. Potempa, M. Lopez, L. T. Braun, J. P. Szidon, L. Fogg, and T. Tincknell, "Physiological outcomes of aerobic exercise training in hemiparetic stroke patients," Stroke, vol. 26, no. 1, pp. 101-105, 1995.

[8] J. O. Kelly, S. L. Kilbreath, G. M. Davis, B. Zeman, and J. Raymond, "Cardiorespiratory fitness and walking ability in subacute stroke patients," Archives of Physical Medicine Rehabilitation, vol. 84, no. 12, pp. 1780$1785,2003$.

[9] M. J. MacKay-Lyons and L. Makrides, "Cardiovascular stress during a contemporary stroke rehabilitation program: Is the intensity adequate to induce a training effect?," Archives of Physical Medicine and Rehabilitation, vol. 83, no. 10, pp. 1378-1383, 2002.

[10] A. Tang, K. M. Sibley, S. G. Thomas, W. E. McIlroy, and D. Brooks, "Maximal exercise test results in subacute stroke," Archives of Physical Medicine and Rehabilitation, vol. 87, no. 8, pp. 1100-1105, 2006.

[11] J. J. Eng, A. S. Dawson, and K. S. Chu, "Submaximal exercise in persons with stroke: Test-retest reliability and concurrent validity with maximal oxygen consumption," Archives of Physical Medicine and Rehabilitation, vol. 85, no. 1, pp. 113-118, 2004.

[12] D. Brooks, A. Tang, K. M. Sibley, and W. E. McIlroy, "Profile of patients at admission into an inpatient stroke rehabilitation programme: cardiorespiratory fitness and functional characteristics," Physiotherapy Canada, vol. 60, no. 2, pp. 171-179, 2008. 
[13] E. J. Roth, K. Mueller, and D. Green, "Stroke rehabilitation outcome - impact of coronary-artery disease," Stroke, vol. 19 , no. 1, pp. 42-47, 1988.

[14] M. Y. C. Pang, J. J. Eng, A. S. Dawson, and S. Gylfadottir, "The use of aerobic exercise training in improving aerobic capacity in individuals with stroke: a meta-analysis," Clinical Rehabilitation, vol. 20, no. 2, pp. 97-111, 2006.

[15] O. Stoller, E. D. de Bruin, R. H. Knols, and K. J. Hunt, "Effects of cardiovascular exercise early after stroke: systematic review and meta-analysis," BMC Neurology, vol. 12, no. 1, p. 45, 2012.

[16] J. K. Chen, T. W. Chen, C. H. Chen, and M. H. Huang, "Preliminary study of exercise capacity in post-acute stroke survivors," Kaohsiung Journal of Medical Sciences, vol. 26, no. 4, pp. 175-181, 2010.

[17] J. S. Yates, S. Studenski, S. Gollub, R. Whitman, S. Perera, S. M. Lai, and P. W. Duncan, "Bicycle ergometry in subacute-stroke survivors: Feasibility, safety, and exercise performance," Journal of Aging and Physical Activity, vol. 12, no. 1, pp. 64-74, 2004.

[18] H. S. Jorgensen, H. Nakayama, H. O. Raaschou, and T. S. Olsen, "Recovery of walking function in stroke patients: the copenhagen stroke study," Arch Phys Med Rehabil, vol. 76, no. 1, pp. 27-32, 1995.

[19] D. T. Wade, V. A. Wood, A. Heller, J. Maggs, and R. L. Hewer, "Walking after stroke - measurement and recovery over the 1st 3 months," Scandinavian Journal of Rehabilitation Medicine, vol. 19, no. 1, pp. 25-30, 1987.

[20] T. G. Hornby, D. H. Zemon, and D. Campbell, "Robotic-assisted, body-weight-supported treadmill training in individuals following motor incomplete spinal cord injury,” Physical Therapy, vol. 85, no. 1, pp. 52-66, 2005.

[21] L. P. Jack, M. Purcell, D. B. Allan, and K. J. Hunt, "The metabolic cost of passive walking during roboticsassisted treadmill exercise," Technology and Health Care, vol. 19, no. 1, pp. 21-27, 2011.

[22] C. Krewer, F. Müller, B. Husemann, S. Heller, J. Quintern, and E. Koenig, "The influence of different lokomat walking conditions on the energy expenditure of hemiparetic patients and healthy subjects," Gait Posture, vol. 26, no. 3, pp. 372-377, 2007.

[23] J. F. Israel, D. D. Campbell, J. H. Kahn, and T. G. Hornby, "Metabolic costs and muscle activity patterns during robotic- and therapist-assisted treadmill walking in individuals with incomplete spinal cord injury," Physical Therapy, vol. 86, no. 11, pp. 1466-1478, 2006.

[24] K. J. Hunt, L. P. Jack, A. Pennycott, C. Perret, M. Baumberger, and T. H. Kakebeeke, "Control of work ratedriven exercise facilitates cardiopulmonary training and assessment during robot-assisted gait in incomplete spinal cord injury," Biomedical Signal Processing and Control, vol. 3, no. 1, pp. 19-28, 2008.

[25] K. J. Hunt and D. B. Allan, "A stochastic hammerstein model for control of oxygen uptake during roboticsassisted gait," International Journal of Adaptive Control and Signal Processing, vol. 23, no. 5, pp. 472-484, 2009.

[26] K. J. Hunt, B. Ajayi, H. Gollee, and L. Jamieson, "Feedback control of oxygen uptake during treadmill exercise," IEEE Transactions on Control Systems Technology, vol. 16, no. 4, pp. 624-635, 2008.

[27] M. Schindelholz and K. J. Hunt, "Feedback control of heart rate during robotics-assisted treadmill exercise," Technology and Health Care, vol. 20, no. 3, pp. 179-194, 2012.

[28] L. P. Jack, M. Purcell, D. B. Allan, and K. J. Hunt, "Comparison of peak cardiopulmonary performance parameters during robotics-assisted treadmill exercise and arm crank ergometry in incomplete spinal cord injury," Technology and Health Care, vol. 18, no. 4-5, pp. 285-296, 2010.

[29] L. Thabane, J. Ma, R. Chu, J. Cheng, A. Ismaila, L. P. Rios, R. Robson, M. Thabane, L. Giangregorio, and C. H. Goldsmith, "A tutorial on pilot studies: the what, why and how," BMC Med Res Methodol, vol. 10, p. 1, 2010. 
[30] W. R. Thompson, N. F. Gordon, and L. S. Pescatello, American College of Sports Medicine. Guidelines for Exercise Testing and Prescription. Philadelphia: Lippincott Williams Wilkins, eight ed., 2010.

[31] M. Schindelholz, O. Stoller, and K. J. Hunt, "A software module for cardiovascular rehabilitation in roboticsassisted treadmill exercise,” Biomedical Signal Processing and Control, vol. 10, pp. 296-307, 2014.

[32] H. Tanaka, K. D. Monahan, and D. R. Seals, "Age-predicted maximal heart rate revisited," Journal of the American College of Cardiology, vol. 37, no. 1, pp. 153-156, 2001.

[33] P. A. Tesch, "Exercise performance and beta-blockade," Sports Medicine, vol. 2, no. 6, pp. 389-412, 1985.

[34] R. A. Robergs, D. Dwyer, and T. Astorino, "Recommendations for improved data processing from expired gas analysis indirect calorimetry," Sports Medicine, vol. 40, no. 2, pp. 95-111, 2010.

[35] B. J. Whipp and K. Wasserman, "Oxygen-uptake kinetics for various intensities of constant-load work," Journal of Applied Physiology, vol. 33, no. 3, pp. 351-356, 1972.

[36] W. L. Beaver, K. Wasserman, and B. J. Whipp, "A new method for detecting anaerobic threshold by gasexchange,” Journal of Applied Physiology, vol. 60, no. 6, pp. 2020-2027, 1986.

[37] P. J. Manns, C. R. Tomczak, A. Jelani, and R. G. Haennel, "Oxygen uptake kinetics: associations with ambulatory activity and physical functional performance in stroke survivors," J Rehabil Med, vol. 42, no. 3, pp. 259-264, 2010 .

[38] B. J. Whipp, S. A. Ward, N. Lamarra, J. A. Davis, and K. Wasserman, "Parameters of ventilatory and gas exchange dynamics during exercise," Journal of Applied Physiology, vol. 52, no. 6, pp. 1506-1513, 1982.

[39] S. K. Powers, S. Dodd, and R. Garner, "Precision of ventilatory and gas exchange alterations as a predictor of the anaerobic threshold," European Journal of Applied Physiology and Occupational Physiology, vol. 52, no. 2, pp. 173-177, 1984.

[40] J. Achten and A. E. Jeukendrup, "Heart rate monitoring - applications and limitations," Sports Medicine, vol. 33, no. 7, pp. 517-538, 2003. 


\section{Chapter 5}

\section{Cardiopulmonary exercise testing early after stroke using feedback-controlled robotics-assisted treadmill exercise: test-retest reliability and repeatability}

Journal of NeuroEngineering and Rehabilitation 2014;11:145 doi: 10.1186/1743-0003-11-145

Oliver Stoller

Eling D de Bruin

Matthias Schindelholz Corina Schuster-Amft Rob A de Bie Kenneth J Hunt 


\section{Abstract}

Background: Exercise capacity is seriously reduced after stroke. While cardiopulmonary assessment and intervention strategies have been validated for the mildly and moderately impaired populations post-stroke, there is a lack of effective concepts for stroke survivors suffering from severe motor limitations. This study investigated the test-retest reliability and repeatability of cardiopulmonary exercise testing (CPET) using feedback-controlled robotics-assisted treadmill exercise (FC-RATE) in severely motor impaired individuals early after stroke.

Methods: 20 subjects (age 44-84 years, $<6$ month post-stroke) with severe motor limitations (Functional Ambulation Classification 0-2) were selected for consecutive constant load testing (CLT) and incremental exercise testing (IET) within a powered exoskeleton, synchronised with a treadmill and a body weight support system. A manual human-in-the-loop feedback system was used to guide individual work rate levels. Outcome variables focussed on standard cardiopulmonary performance parameters. Relative and absolute test-retest reliability were assessed by intraclass correlation coefficients (ICC), standard error of the measurement (SEM), and minimal detectable change (MDC). Mean difference, limits of agreement, and coefficient of variation (CoV) were estimated to assess repeatability.

Results: Peak performance parameters during IET yielded good to excellent relative reliability: absolute peak oxygen uptake ( $\mathrm{ICC}=0.82$ ), relative peak oxygen uptake $(\mathrm{ICC}=0.72)$, peak work rate ( $\mathrm{ICC}=0.91$ ), peak heart rate ( $\mathrm{ICC}=0.80)$, absolute gas exchange threshold $(\mathrm{ICC}=0.91)$, relative gas exchange threshold (ICC $=0.88$ ), oxygen cost of work ( $\mathrm{ICC}=0.87$ ), oxygen pulse at peak oxygen uptake ( $\mathrm{ICC}=0.92$ ), ventilation rate versus carbon dioxide output slope $(\mathrm{ICC}=0.78)$. For these variables, SEM was 4-13\%, MDC 12-36\%, and CoV 10-36\%. CLT revealed high mean differences and insufficient test-retest reliability for all variables studied.

Conclusions: This study presents first evidence on reliability and repeatability for CPET in severely motor impaired individuals early after stroke using a feedback-controlled robotics-assisted treadmill. The results demonstrate good to excellent test-retest reliability and appropriate reproducibility for the most important peak cardiopulmonary performance parameters. These findings have important implications for the design and implementation of cardiovascular exercise interventions in severely impaired populations. Future research needs to develop advanced control strategies to enable the true limit of functional exercise capacity to be reached and to further assess test-retest reliability and repeatability in larger samples. 


\section{Background}

Exercise capacity and activity status have become well-established predictors of cardiovascular and overall mortality, both of which are seriously reduced after stroke [1,2]. It has been shown that peak oxygen uptake ( $\dot{\mathrm{V}}_{2}$ peak) is approximately $50 \%$ lower compared to normative values of healthy adults 30 days post-stroke [3,4]. Despite extensive inpatient rehabilitation procedures and spontaneous recovery of cardiovascular fitness, the exercise capacity of stroke survivors entering the chronic phase remains below recommended levels [5]. The rapid deterioration of fitness not only predisposes to secondary medical complications, but also restricts the degree to which individuals can participate in rehabilitation routines and limits the ability of the individual to perform functional activities independently [6]. Therefore, research into cardiovascular exercise training in the early stages after stroke has been highlighted as a priority [7, 8]. Effective assessment and intervention strategies are needed to assess, monitor, and improve cardiovascular fitness early after stroke.

Current research has investigated several modalities for cardiopulmonary exercise testing $(\mathrm{C}$ PET) in subacute stroke (6 days- 6 months post-stroke) $[3,4,9,10,11,12,13]$ and in chronic stroke (>6 months post-stroke) $[14,15,16,17,18,19]$ using treadmill exercise $[14,15,16]$, body weight supported treadmill exercise [3], leg cycle ergometry [4, 9, 10,11, 15, 17, 18], and combined upperand lower-limb ergometry $[12,19]$. The most common concepts, i.e. treadmill exercise and leg cycle ergometry, are primarily designed for individuals with mild to moderate motor impairment, because limited motor control (non-ambulatory status, limited trunk control), poor postural control, and poor coordination of the affected limbs may restrict severely impaired individuals from performing on these devices. As a result, most studies focussing on exercise capacity after stroke have excluded individuals not able to walk independently and those presenting with low levels of motor function.

A potential option to overcome severe motor restrictions is the introduction of combined upper- and lower-limb ergometry [12, 19, 20]. Current study results demonstrated feasibility and validity, and emphasised the fact that an all-extremity exercise protocol might decrease early onset of lower limb fatigue which leads to better estimates of exercise capacity due to the incorporation of more muscle mass. However, this approach does not embody the concept of repetitive taskspecific exercise during the early stages of stroke recovery and might be not appropriate for implementation into early rehabilitation phases [21, 22]. Considering the relatively short intervention window during subacute stroke rehabilitation and the current recommendations for cardiovascular exercise training after stroke [8], novel approaches should incorporate task-specific activities such 
as walking or stair climbing. The combination of motor function training and cardiovascular exercise might have the potential to positively influence overall therapy outcomes and to prevent or mitigate the loss of exercise capacity in the early stages after stroke onset [23].

A promising approach to overcome motor limitations while facilitating task-specific activity and cardiovascular stress is body weight supported treadmill training. Initial research has shown that gait symmetry improved with increasing body weight support (BWS) [24]. However, during walking with BWS of more than $15 \%$, vertical ground reaction forces and functional activity of antigravity muscles decreased, which led to substantially lower oxygen uptake levels during body weight supported treadmill training compared to conventional treadmill exercise $[25,26]$. Because severely impaired stroke survivors need considerable physical support during walking with low body weight support, the application of robotics-assisted treadmill exercise (RATE) might be of relevance in this context. A powered exoskeleton for the lower extremities, synchronised with a treadmill and BWS, provides active support during the gait trajectory that enables progressive body weight loading for individuals with severe motor restrictions.

Recent research on exercise intensity during RATE has shown substantial increases in cardiopulmonary performance parameters after stroke [27, 28], and spinal cord injury [29], including complete tetraplegia [30]. However, oxygen uptake levels were below that of overground walking, recommended cardiovascular training intensities could not be achieved [31], and conventional control strategies such as the modulation of walking speed, BWS, and guidance force had only a minor influence on exercise intensity [27, 28, 31]. There is a need for voluntary effort during walking within an exoskeleton to provoke substantial cardiovascular stress comparable to conventional treadmill exercise [32]. Therefore, novel protocols have been developed to control and direct active participation during RATE with the specific aim of provoking cardiorespiratory responses $[33,34,35,36,37,38]$. This incorporates biofeedback mechanisms allowing the control of exercise intensity through the guidance of the individuals voluntary effort. The approach presented here provides control of exercise intensity during RATE by biofeedback and voluntary adaptation of the hip and knee forces by the subject. A first clinical study in non-ambulatory stroke survivors in the subacute phase revealed that feedback-controlled RATE (FC-RATE) can be used to implement CPET [39]. Results yielded acceptable cardiopulmonary performance parameters following standardised CPET protocols. Thus, this approach might have the potential to assess exercise capacity and guide cardiovascular exercise in stroke survivors with severe motor limitations. This needs to be formally investigated for clinical feasibility, test-retest reliability and repeatability. 
The aims of this study were: (1) to assess the clinical feasibility of FC-RATE for CPET in severely motor impaired individuals early after stroke, (2) to examine the ability of the concept to meet standard cardiopulmonary criteria for maximal exercise capacity, and (3) to assess the test-retest reliability and the repeatability of the approach.

\section{Methods}

\section{Participants}

20 first-ever stroke inpatients were recruited at a neurological rehabilitation clinic in the northwestern part of Switzerland (Reha Rheinfelden) and screened according to the selection criteria. Subjects were then presented to the responsible ward physician and a cardiologist to confirm eligibility. Inclusion criteria were: (1) clinical diagnosis of initial stroke (ischaemic or haemorrhagic), (2) $<20$ weeks after stroke onset, (3) age $>18$ years, (4) Functional Ambulation Classification (FAC) of $<3,(5)$ ability to understand the procedures and provide informed consent. Subjects were excluded if they had (1) cardiac contraindications for exercise testing according to the American College of Sports Medicine (ACSM) [40], (2) contraindications for RATE according to guidelines from the manufacturer (Hocoma AG, Volketswil, Switzerland), (3) concurrent neurological disease (e.g. Multiple Sclerosis, Parkinsons Disease, etc.), (4) concurrent pulmonary disease (e.g. COPD, etc.), (5) history of dementia.

Recorded characteristics included gender, age, body mass index, diagnosis, affected body side, time post-stroke, medications, comorbidities, FAC [41] and functional independence using the Extended Barthel Index (EBI) [42]. All subjects were informed about risks and benefits, and gave signed informed consent. The Ethics Review Committee of the Swiss canton of Aargau approved the study (Reference No: 2012/051).

\section{Technical implementation}

The Lokomat system (Hocoma AG, Volketswil, Switzerland) was used to implement FC-RATE. The powered exoskeleton provides control of both legs using DC motors, synchronised with an integrated treadmill ( $\mathrm{h} / \mathrm{p} / \mathrm{cosmos}$ sports medical GmbH, Traunstein, Germany) and a motor-driven BWS system with real time feedback control for precise body weight unloading (Lokolift, Hocoma AG). The total mechanical work rate exerted on the exoskeleton by the subject was computed from the force, moment arm and velocity data at the four active joints (hips and knees). The active mechanical work rate $\left(\mathrm{P}_{\text {mech }}\right)$, applied by the subjects effort was estimated by subtracting 
the passive mechanical work rate (work rate necessary to move the subject passively within the exoskeleton) from the total mechanical work rate. A manual human-in-the-loop feedback system was implemented to control the subjects active work rate. $\mathrm{P}_{\text {mech }}$ was projected onto a screen at the front of the treadmill together with a target mechanical work rate $\mathrm{P}_{\text {mech }}^{*}$. The subject was instructed to vary the forces applied on the exoskeleton by volitional muscle activity and to keep the measured and visualised active work rate as close as possible to the target (Figure 5.1).

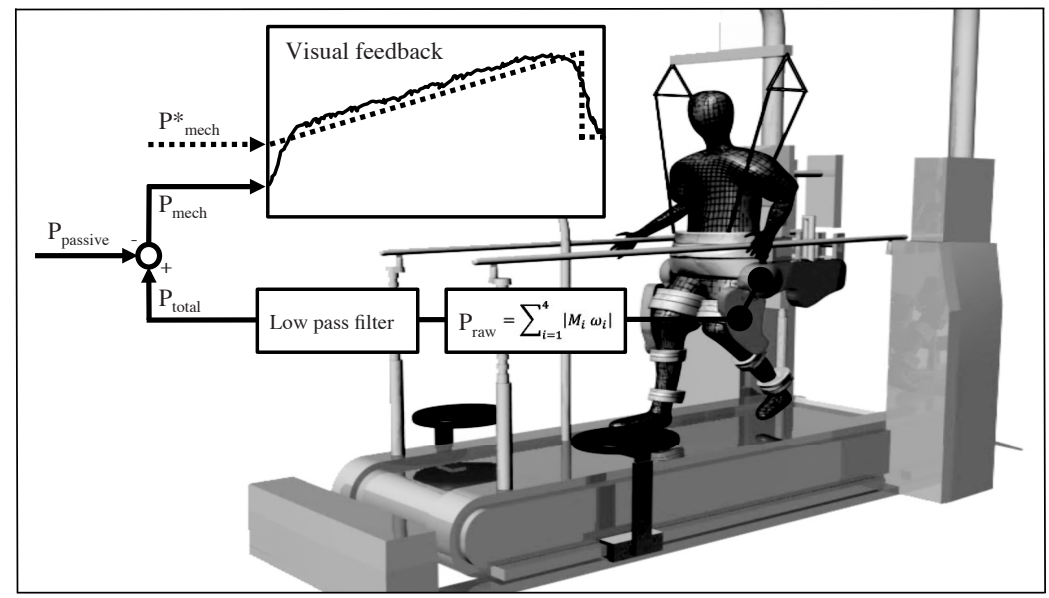

Figure 5.1: Feedback-controlled robotics-assisted treadmill exercise. Hip and knee joint forces and angles are measured in real time to allow calculation of the mechanical work rate $\left(\mathrm{P}_{\text {mech }}\right.$, solid line $)$ and projection onto a screen in front of the subject. Individual target work rate profiles $\left(\mathrm{P}_{\text {mech }}^{*}\right.$, dashed line) are used to guide exercise intensity during robotics-assisted walking. The passive mechanical work rate $\left(\mathrm{P}_{\text {passive }}\right)$ is evaluated before every session and subtracted from $\mathrm{P}_{\text {mech. }}$. Legend: $\mathrm{P}_{\text {raw }}=$ raw mechanical work rate, $M_{i}=$ moments of force, $\omega_{i}=$ angular velocity, $\mathrm{P}_{\text {total }}=$ total mechanical work rate.

\section{Experimental protocol}

At study entry, all included subjects completed a familiarisation session with the FC-RATE concept, which started by qualified and experienced physiotherapists adjusting the Lokomat system to provide a physiological gait pattern and to ensure that the subjects could walk comfortably. Then, an initial test of decreasing BWS continuously by $5 \%$ per minute was implemented to define the minimal possible BWS level. There was strict adherence to physiological gait pattern criteria 
through visual observation: (1) heel strike (physiological knee extension), (2) no foot dragging during the swing phase, and (3) active weight-bearing during the stance phase (physiological knee extension) [43]. After the first adjustments, subjects were asked to perform a short constant load exercise test for $5 \mathrm{~min}\left(\mathrm{P}_{\text {mech }}^{*}=20 \mathrm{~W}\right)$ to explain the approach and practice with the feedbackcontrol structure. Finally, the safety procedures for potential adverse events were explained in detail.

After a break of at least $24 \mathrm{~h}$, subjects then completed repeated constant load testing (CLT) and incremental exercise testing (IET) on separate days, with 48-72 h between the trials. All sessions were controlled for time of day. Subjects were instructed to avoid additional strenuous activity during participation in the study and not to consume food, alcohol, nicotine or caffeine at least $3 \mathrm{~h}$ prior to testing.

Subjects were asked at the beginning of the first CLT and IET to increase their maximal voluntary effort during RATE within $30 \mathrm{~s}$ to define the maximal work rate (Pmax) for the subsequent tests. Walking cadence was fixed at $60 \mathrm{steps} / \mathrm{min}$ and individual BWS was consistent for all sessions. An experienced examiner performed all tests. There was close adherence to established models for exercise testing according to the ACSM guidelines [39].

CLT was based on constant-intensity exercise (40\% Pmax) separated into 4 phases: (1) rest - subjects stood on the treadmill for 5 min with $0 \%$ BWS, (2) passive phase - subjects walked passively with their individual BWS for 5 min, (3) active phase - subjects actively contributed to the walking by pushing forward within the exoskeleton during the swing phase of each leg to reach the target work rate for $10 \mathrm{~min}$, (4) recovery - subjects walked passively with their individual BWS for $5 \mathrm{~min}$ (Figure 5.2A). IET was based on progressive ramp exercise and separated into 4 phases: (1) rest - subjects stood on the treadmill for 5 min with $0 \%$ BWS, (2) passive phase subjects walked passively with their individual BWS for $5 \mathrm{~min}$, (3) active phase - subjects actively contributed to the walking by pushing forward within the exoskeleton during the swing phase of each leg to reach the target work rate, (4) recovery - subjects walked passively with their individual BWS for $5 \mathrm{~min}$. The progressive ramp (active phase) was defined as a continuous slope aiming to the reach predefined Pmax in 10 min (Figure 5.2B).

Both test protocols followed strict termination criteria for CPET including: (1) abnormal blood pressure responses, i.e. hypertensive (systolic $>210 \mathrm{mmHg} /$ diastolic $>115 \mathrm{mmHg}$ ) when exercising at high work rate, or hypotensive responses (decrease of $>10 \mathrm{mmHg}$ ) despite an increase in work rate, (2) individual work rate below target work rate for $60 \mathrm{~s}$, (3) peak heart rate within 10 beats per minute of the age-predicted heart rate maximum [44], where the formula was adjusted down to $70 \%$ of heart rate maximum for subjects on beta-blocker medications [45], (d) pain or dis- 
comfort. Subjects rated their perceived exertion using the Borg rating of perceived exertion scale $(\mathrm{RPE})(6=$ no exertion at all, $20=$ maximal exertion $)$ [46].

Several risk management strategies were implemented to ensure subjects safety: (1) clearly defined eligibility criteria to include medically stable subjects only, (2) screening by cardiologists to exclude subjects with potential risk factors (i.e. abnormalities in resting ECG, history of any cardiac/cardiovascular disease, uncontrolled metabolic disease), (3) continuous blood pressure and heart rate monitoring during exercise testing, (4) presence of resuscitation-trained assistants, (5) opportunity to call the emergency medical resuscitation team in the clinic, and (6) presence of personnel trained to release the subject within $60 \mathrm{~s}$ from the exoskeleton. Detailed information on FC-RATE-based CPET can be found elsewhere [39, 47].

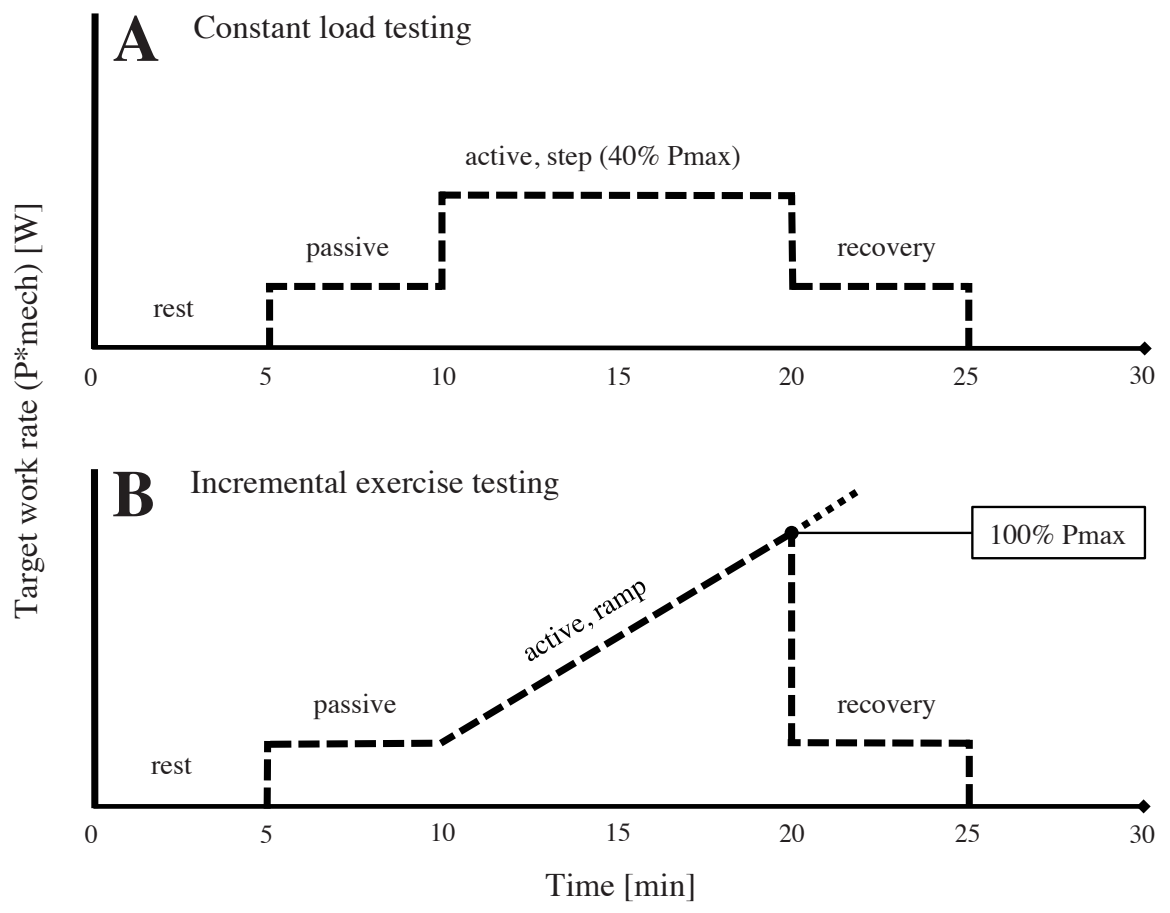

Figure 5.2: Exercise testing protocols. Schematic representation of constant load testing, CLT, (A) and incremental exercise testing, IET, (B) using feedback-controlled robotics-assisted treadmill exercise. The dashed line represents the target work rate $\left(\mathrm{P}_{\mathrm{mech}}^{*}\right)$. The slope during incremental exercise testing was estimated such that the predefined work rate maximum $(\mathrm{P} \max )$ was reached at $10 \mathrm{~min}$ during the active phase. When individual termination criteria were met the incremental phase was ended and $\mathrm{P}_{\text {mech }}^{*}$ set back to the passive level (recovery). 


\section{Outcomes}

Measured cardiopulmonary performance parameters were: oxygen uptake $\left(\dot{\mathrm{V}} \mathrm{O}_{2}\right)$, carbon dioxide output $\left(\dot{\mathrm{V}} \mathrm{CO}_{2}\right)$, ventilation rate $\left(\dot{\mathrm{V}}_{\mathrm{E}}\right)$, respiratory rate $\left(\mathrm{R}_{\mathrm{f}}\right)$, and heart rate $(\mathrm{HR})$. These were recorded by a breath-by-breath cardiorespiratory monitoring system (MetaMax 3B, Cortex Biophysik, Leipzig, Germany), including a heart rate belt (T31, Polar Electro, Kempele, Finland) and a receiver board (HRMI, Sparkfun, Boulder, USA). Pmech was calculated using the exoskeleton geometry and interaction forces, and angular signals, which were available in real time from a custom interface unit.

For CLT, outcome variables were speed of oxygen uptake kinetics (time constant $\tau$ ), oxygen cost of passive walking ( $\Delta$ rest vs. passive walking), oxygen cost of active walking ( $\Delta$ passive walking vs. active walking), and accuracy of work rate tracking (RMSE $\mathrm{P}_{\mathrm{P}}$. IET focused on peak performance parameters for oxygen uptake $\left(\dot{\mathrm{V} O}{ }_{2}\right.$ peak), time to $\dot{\mathrm{V}} \mathrm{O}_{2}$ peak ( $\mathrm{tVO}_{2}$ peak), work rate (Ppeak), ventilation rate $\left(\dot{\mathrm{V}}_{\mathrm{E}}\right.$ peak), respiratory rate $\left(\mathrm{R}_{\mathrm{f}}\right.$ peak), heart rate (HRpeak), and respiratory exchange ratio (RERpeak). In addition, gas exchange threshold (GET), oxygen cost of work $\left(\Delta \dot{\mathrm{V} O} \mathrm{O}_{2} / \Delta \mathrm{P}\right), \mathrm{O}_{2}$ pulse at $\dot{\mathrm{V}} \mathrm{O}_{2}$ peak $\left(\mathrm{O}_{2}\right.$ pulse $), \dot{\mathrm{V}}_{\mathrm{E}}$ versus $\dot{\mathrm{VCO}}_{2}$ slope $\left(\Delta \dot{\mathrm{V}}_{\mathrm{E}} / \Delta \dot{\mathrm{VCO}}_{2}\right)$, and $\mathrm{RMSE}_{\mathrm{P}}$ were evaluated.

\section{Data processing}

Raw breath-by-breath data were processed using a zero phase shift moving average filter over 15 breaths [48]. For CLT, the time constant for the oxygen uptake kinetics $\tau$ was calculated using a non-linear least-squares algorithm to fit the data as described in the following mono-exponential equation: $\dot{\mathrm{VO}}_{2}(\mathrm{t})=\dot{\mathrm{V}} \mathrm{O}_{2}(\mathrm{~b})+\dot{\mathrm{V}} \mathrm{O}_{2}\left(1-\mathrm{e}^{-(\mathrm{t}-\mathrm{Td}) / \tau}\right), \mathrm{t}<0$, with $\dot{\mathrm{V}}_{2}(\mathrm{~b})=$ oxygen uptake at baseline, $\Delta \dot{\mathrm{VO}_{2}}=$ step increase in oxygen uptake, $\mathrm{Td}=$ time delay of $20 \mathrm{~s}$ corresponding to the cardio-dynamic phase of the response, and $\tau=$ time constant [49]. Steady-state was defined by excluding the first 2 minutes and last minute of each phase, i.e. steady-state calculations were done using data from the $3^{r d}-4^{\text {th }}$ minute of a given phase. Cost of passive walking was defined as the difference between rest and passive steady-state values, whereas cost of active walking was estimated from the difference between passive and active steady-states. For IET, peak cardiopulmonary response variables were defined as the maximal values in the final $30 \mathrm{~s}$ during the incremental phase. Criteria for maximal aerobic capacity were (1) plateau in oxygen uptake, (2) respiratory exchange ratio $(\mathrm{RER}) \geq 1.15$, and (3) peak heart rate within 10 beats per minute of the age-predicted heart rate maximum (adjusted for subjects on beta-blocker medications) [40]. The identification of a plateau or reduction in $\dot{\mathrm{VO}}_{2}$ was performed by plotting the slope and $95 \%$ con- 
fidence interval (CI) of the $\dot{\mathrm{V}} \mathrm{O}_{2}-\mathrm{P}_{\text {mech }}$ slope by least-squares linear regression analysis, where the presence of data points that fell below and outside the extrapolated 95\% CI were taken as evidence of plateauing or levelling-off behaviour [50]. The GET was estimated using the v-slope method, where the anaerobic threshold is identified as the deflection point of the $\dot{\mathrm{V}} \mathrm{O}_{2}-\dot{\mathrm{V} C \mathrm{CO}_{2}}$ relationship [51]. The accuracy of work rate tracking $\left(\mathrm{RMSE}_{\mathrm{P}}\right)$ was expressed by the root mean square error between $\mathrm{P}_{\text {mech }}$ and $\mathrm{P}_{\text {mech }}^{*}$. Data processing was performed using MATLAB (Version R2010a, MathWorks, Natick MA, USA) and LabVIEW (Version 2009, National Instruments, Austin TX, USA).

\section{Statistical analysis}

Descriptive statistics were calculated for all outcome variables. Due to the small sample size, Wilcoxon-tests were applied to exclude significant practice effects. Test-retest reliability was quantified using intraclass correlation coefficients $\left(\mathrm{ICC}_{3,1}\right)$ with $95 \% \mathrm{CI}$. The ICC provides an estimate of the relative reliability of measurement when the population under study is heterogeneous [52]. ICC results of 0.60-0.74 were considered as "good", and ICC results $>0.74$ as "excellent" [53]. Absolute reliability was determined by estimating the standard error of measurement (SEM $=$ standard deviation of the difference (SDdiff) $\sqrt{1-I C C}$ ) and the minimal detectable change $(\mathrm{MDC}=1.96 \times \sqrt{2} \times \mathrm{SEM})$, presented in absolute values and percentages [54, 55]. Repeatability was estimated by mean difference (MD), limits of agreement (LoA) (MD $\pm 1.96 \mathrm{x}$ SDdiff), and coefficients of variation (CoV) (SDdiff / mean). Two-sided $\mathrm{p}$-values $\mathrm{p} \leq 0.05$ were considered significant. Statistical analyses were performed using SPSS (Version 20.0, IBM, Armonk NY, USA) and MATLAB (Version R2010a, MathWorks, Natick MA, USA).

\section{Results}

\section{General observations}

Of the 20 subjects enrolled in the study, 1 subject showed an abnormal gait pattern due to uncontrollable spasticity during familiarisation and 1 subject developed a tibia skin lesion due to inadequate padding of the exoskeleton, which led to withdrawal from the study (Figure 5.3). Further, 4 withdrawals after the first IET occurred due to groin pain, lack of motivation, liquor swelling, and acute respiratory infection. Thus, 18 subjects (90\%) performed the two CLTs and, of these, $14(70 \%)$ also performed the two IETs. All subjects presented with severe motor impairments and 
were non-ambulatory (FAC range 0-2). BWS ranged between 46-77\% and walking speed was set at $60 \mathrm{steps} /$ minute $(0.47-0.67 \mathrm{~m} / \mathrm{s})$. The subject characteristics are summarised in Table 5.1 .

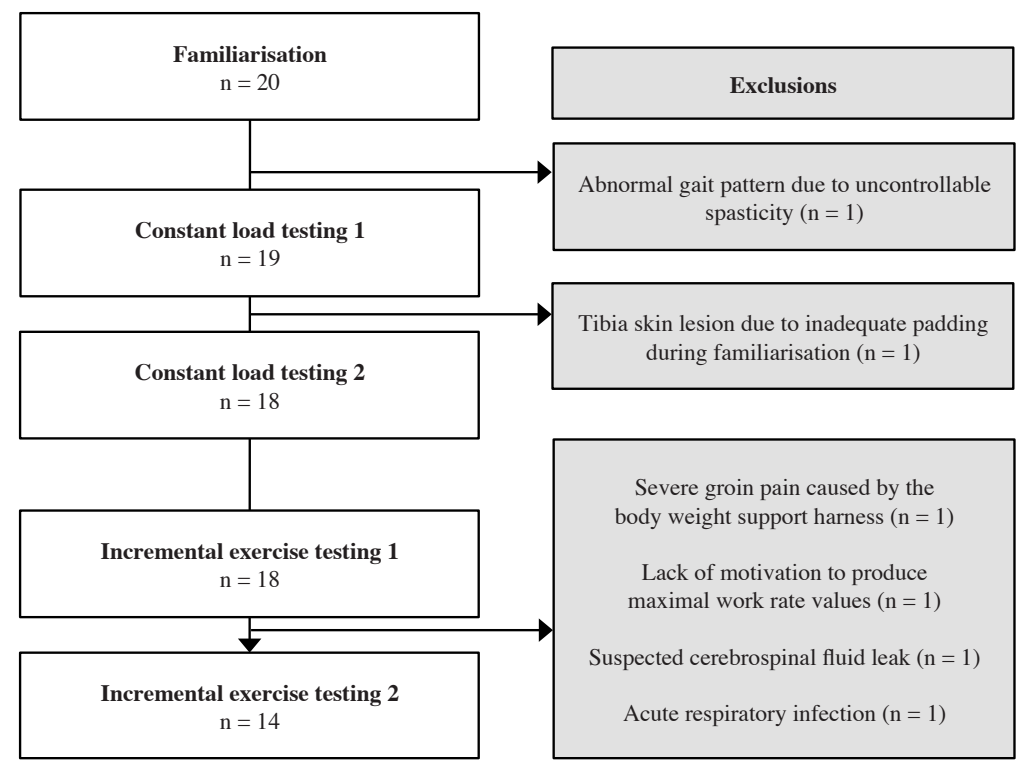

Figure 5.3: Study flow chart.

All subjects successfully completed the predefined CPET protocol (rest, passive, active, recovery). During CLT, 3 subjects stopped the active walking phase after 5 minutes due to generalised fatigue and continued with the recovery phase. All other CLT conditions were performed according to the plan. 13 subjects completed both IETs without symptomatic responses requiring termination per safety criteria; the reason for test termination was in every case the inability to reach $\mathrm{P}_{\text {mech }}$ due to generalised and/or leg fatigue. The examiner stopped 2 consecutive IET sessions in 1 subject due to high blood pressure responses according to the safety criteria ( 2 adverse events); however, no serious adverse events occurred during testing. Mean IET duration was 23.2 \pm 2.6 min (active phase: $8.2 \pm 2.6 \mathrm{~min})$. RPE at peak performance was $14.8 \pm 1.9$. $\mathrm{RMSE}_{\mathrm{P}}$ values were $<10 \mathrm{~W}$. 
Table 5.1: Subject characteristics

\section{Constant load testing}

$\mathrm{n}=18$

$11 / 7$

$12 / 6$

$9 / 9$

$49 \pm 31(14-139)$

$61 \pm 11(44-84)$

$27 \pm 5(19-38)$

$1.1 \pm 0.8(0-2)$

$43 \pm 9(27-56)$

EBI (0-64)

Medications: beta-blockers / ACE inhibitors / both

Comorbidities:ypertension / Dyslipi-

demia / Adipositas / Diabetes mellitus

BWS [\%]

Walking speed $[\mathrm{m} / \mathrm{s}]$

RPE (6-20)

Pmax during MVC-W [W]

\section{$6 / 11 / 4$}

$9 / 6 / 3 / 3$

$59 \pm 9(46-77)$

$0.57 \pm 0.05(0.47-0.67)$

$13 \pm 2(6-17)$

$38.4 \pm 23.0(8.5-77.2)$
Incremental exercise testing

$\mathrm{n}=14$

$9 / 5$

$13 / 3$

$8 / 6$

$43 \pm 25$ (14-92)

$61 \pm 12(44-84)$

$28 \pm 6(19-38)$

$0.9 \pm 0.8(0-2)$

$42 \pm 9(27-55)$

$4 / 11 / 4$

$8 / 6 / 3 / 3$

$60 \pm 9(46-77)$

$0.56 \pm 0.05(0.47-0.64)$

$15 \pm 2(11-18)$

$57.1 \pm 33.1(11.3-127.7)$

Values are given in numbers $(\mathrm{n})$ or mean \pm standard deviation (range)

Abbreviations: $\mathrm{BMI}=$ Body Mass Index, $\mathrm{FAC}=$ Functional Ambulation Classification, EBI $=$ Extended Barthel Index, ACE inhibitors: Angiotensin-converting enzyme inhibitors, BWS: Body weight support, RPE: Rate of perceived exertion, Pmax: Maximal work rate (power), MVC-W: Maximal voluntary contraction during walking

\section{Exercise capacity}

For CLT, time constants of oxygen uptake kinetics $(\tau)$ could not be evaluated due to continuous disturbances of $\dot{\mathrm{VO}}_{2}$ in the transition phases (rest/passive walking, passive walking/active walking). Cost of passive walking ( $\Delta$ rest vs. passive walking) was (mean \pm standard deviation): $\dot{\mathrm{VO}}_{2}$ $=184.2 \pm 124.0 \mathrm{~mL} / \mathrm{min}, \mathrm{HR}=1.4 \pm 6.4$ beats $/ \mathrm{min}$, and cost of active walking $(\Delta$ passive walking vs. active walking) was: $\dot{\mathrm{VO}_{2}}=45.7 \pm 56.6 \mathrm{~mL} / \mathrm{min}, \mathrm{HR}=2.3 \pm 3.1$ beats $/ \mathrm{min}$. RMSE during CLT was $5.5 \pm 5.5 \mathrm{~W}$. For IET, peak performance parameters were: absolute $\dot{\mathrm{V}} \mathrm{O}_{2}$ peak $=1280.9 \pm 564.8 \mathrm{~mL} / \mathrm{min}$, relative $\dot{\mathrm{V}}{ }_{2}$ peak $=15.5 \pm 4.9 \mathrm{~mL} / \mathrm{min} / \mathrm{kg}(51.6 \pm 20.5 \%$ of predicted $\left.\dot{\mathrm{VO}}_{2} \max [56]\right), \mathrm{tVO}_{2}$ peak $=8.2 \pm 2.6 \mathrm{~min}$, Ppeak $=53.9 \pm 33.8 \mathrm{~W}, \dot{\mathrm{V}}_{\mathrm{E}}$ peak $=41.3 \pm 18.6 \mathrm{~L} / \mathrm{min}$, $\mathrm{R}_{\mathrm{f}}$ peak $=36.1 \pm 8.31 / \mathrm{min}$, HRpeak $=126.0 \pm 19.5$ beats $/ \mathrm{min}(84.3 \pm 12.2 \%$ of age-predicted heart 
rate maximum [44]), and RERpeak $=0.92 \pm 0.09$. Absolute GET was at a $\dot{\mathrm{VO}}{ }_{2}$ of $878.9 \pm 316.6$ $\mathrm{mL} / \mathrm{min}$ and relative $\mathrm{GET}$ at $11.0 \pm 3.1 \mathrm{~mL} / \mathrm{min} / \mathrm{kg}$, which was $\mathrm{GET} \%=72.6 \pm 12.2 \%$ of $\dot{\mathrm{V}} \mathrm{O}_{2}$ peak. $\Delta \dot{\mathrm{V} O}{ }_{2} / \Delta \mathrm{P}$ was $20.1 \pm 14.4 \mathrm{~mL} / \mathrm{W}, \mathrm{O}_{2}$ pulse was $10.2 \pm 4.1 \mathrm{~mL} /$ beat, and $\Delta \dot{\mathrm{V}}_{\mathrm{E}} / \Delta \dot{\mathrm{V} C O} \mathrm{O}_{2}$ was 36.3 \pm 7.3L. RMSE $\mathrm{P}_{\mathrm{P}}$ during IET was $8.7 \pm 9.0 \mathrm{~W}$.

With respect to the 3 criteria for maximal aerobic capacity, 2 subjects $(14 \%)$ showed a plateau in $\dot{\mathrm{VO}}_{2}$ at the end of IET, 1 subject (7\%) achieved an RER value $\geq 1.15$, and 5 subjects (36\%) reached peak heart rate within 10 beats per minute of the age-predicted heart rate maximum, where 2 of these subjects had an adjusted heart rate due to beta-blockers. Thus, $57 \%$ of the subjects achieved at least 1 of the 3 criteria for maximal exercise capacity.

\section{Test-retest reliability and repeatability}

Table 5.2 shows mean values, test-retest reliability and repeatability results of the repeated CLT and IET trials. No practice effects could be detected; trials were not significantly different. Outcome variables for CLT yielded high MD between tests and insufficient test-retest reliability and repeatability throughout. For IET, good to excellent relative reliability was found for absolute $\dot{\mathrm{V}} \mathrm{O}_{2}$ peak $(\mathrm{ICC}=0.82)$, relative $\dot{\mathrm{V}}{ }_{2}$ peak $(\mathrm{ICC}=0.72)$, Ppeak $(\mathrm{ICC}=0.91)$, HRpeak $(\mathrm{ICC}=$ $0.80)$, absolute GET (ICC $=0.91)$, relative GET $(\mathrm{ICC}=0.88), \Delta \dot{\mathrm{V}} \mathrm{O}_{2} / \Delta \mathrm{P}(\mathrm{ICC}=0.87), \mathrm{O}_{2}$ pulse $(\mathrm{ICC}=0.92)$, and $\Delta \dot{\mathrm{V}}_{\mathrm{E}} / \Delta \dot{\mathrm{VCO}}_{2}(\mathrm{ICC}=0.78)$. SEM were between $4-13 \%$ and MDC ranged from 12-36\%. $\mathrm{MD} \pm$ SDdiff of the outcome variables that were analysed for relative reliability were: absolute $\dot{\mathrm{V}}{ }_{2}$ peak $=45.5 \pm 353.7 \mathrm{~mL} / \mathrm{min}$, relative $\dot{\mathrm{VO}_{2}}$ peak $=1.0 \pm 3.8 \mathrm{~mL} / \mathrm{min} / \mathrm{kg}$, Ppeak $=2.4 \pm 15.0 \mathrm{~W}$, HRpeak $=3.6 \pm 12.6$ beats $/ \mathrm{min}$, absolute GET $=67.3 \pm 124.2 \mathrm{~mL} / \mathrm{min}$, relative $\mathrm{GET}=0.2 \pm 1.6 \mathrm{~mL} / \mathrm{min} / \mathrm{kg}, \Delta \dot{\mathrm{VO}} \mathrm{O}_{2} / \Delta \mathrm{P}=2.7 \pm 7.2 \mathrm{~mL} / \mathrm{min} / \mathrm{W}, \mathrm{O}_{2}$ pulse $=0.1 \pm 1.7 \mathrm{~mL} / \mathrm{beat}$, $\Delta \dot{\mathrm{V}}_{\mathrm{E}} / \Delta \dot{\mathrm{VCO}}_{2}=0.5 \pm 5.1 \mathrm{~L} . \mathrm{CoV}$ for peak cardiopulmonary performance parameters ranged from $10-44 \%$. Bland-Altman plots for the major outcome variables visualise the differences between tests (Figure 5.4 and 5.5). 


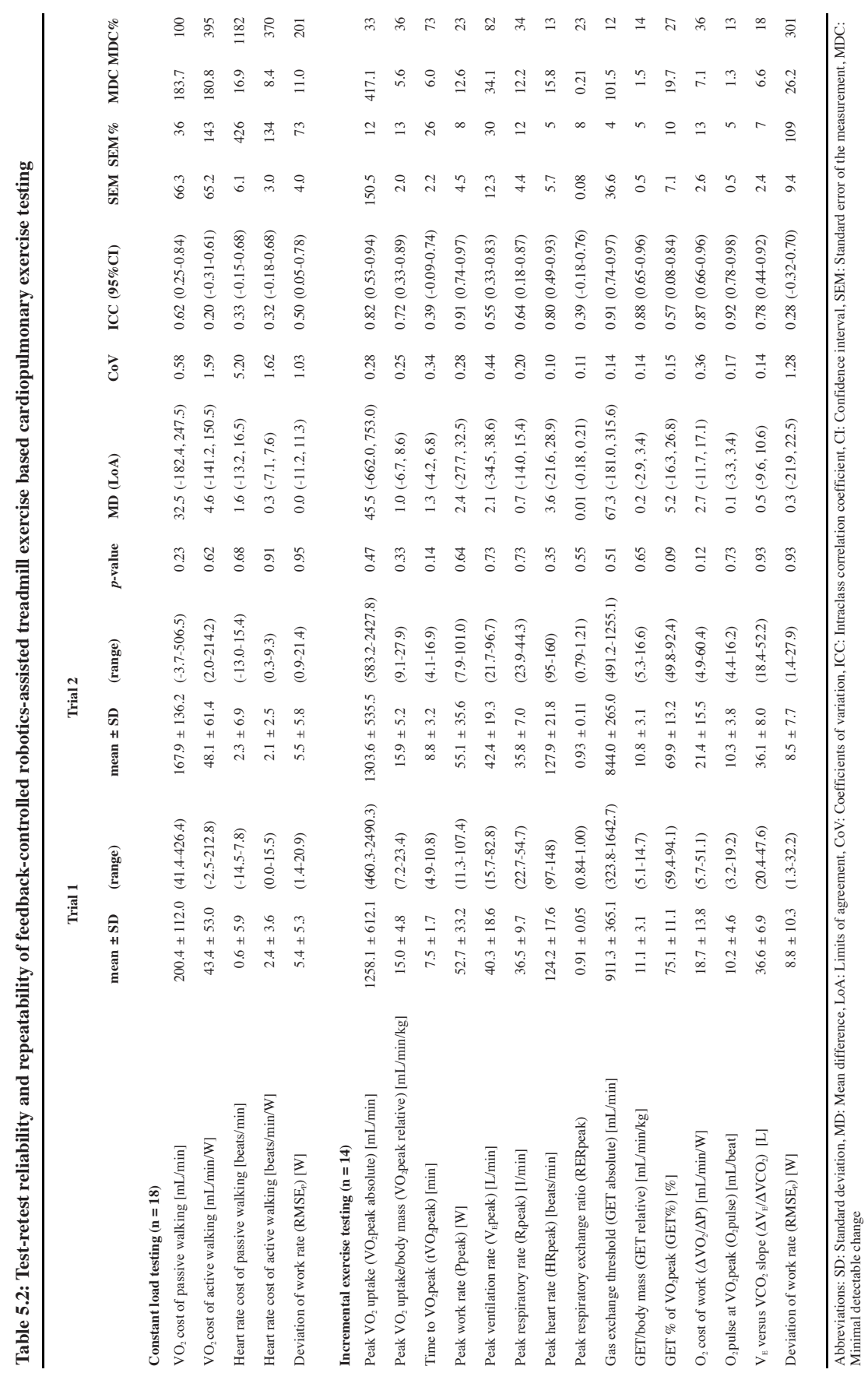



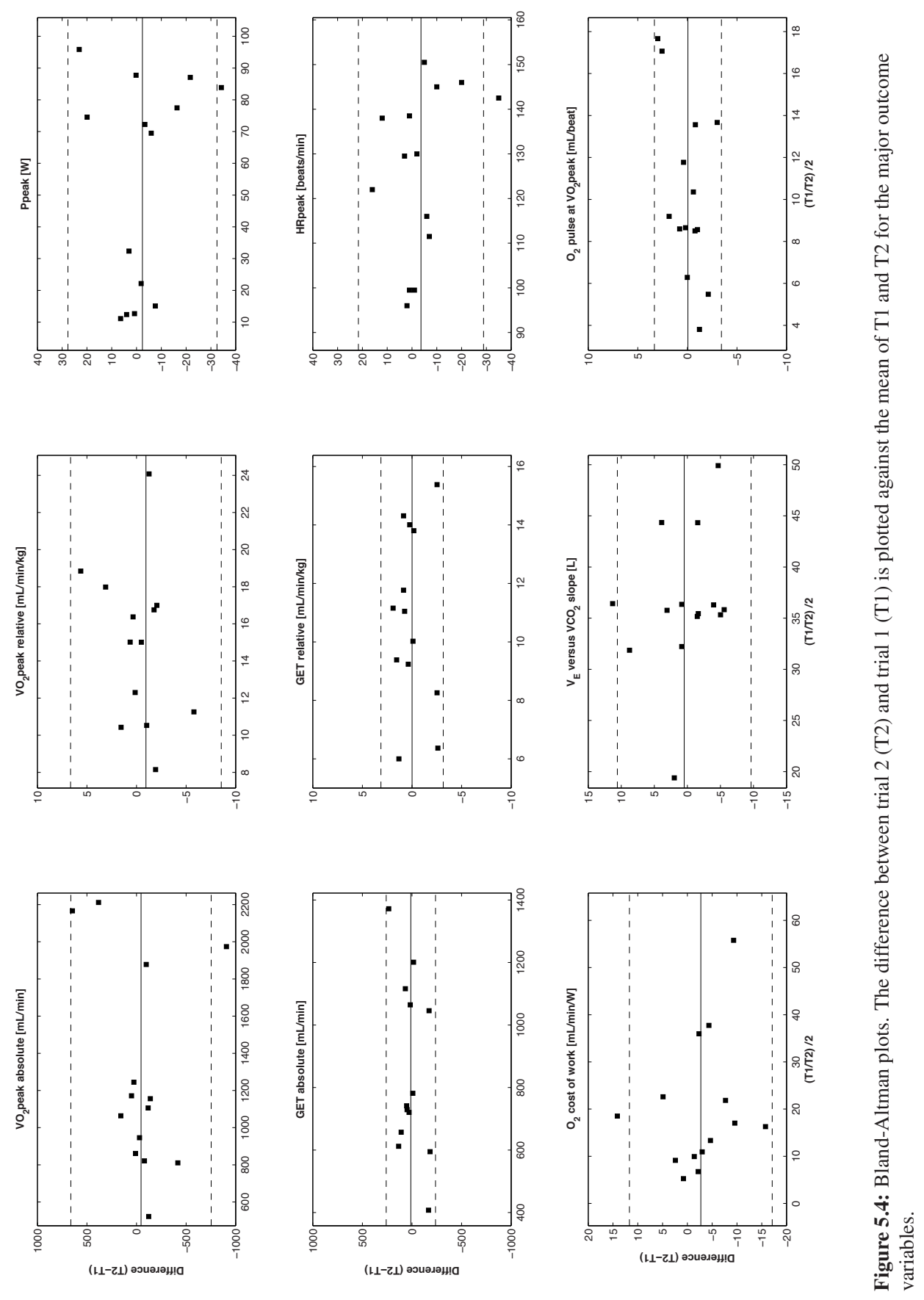

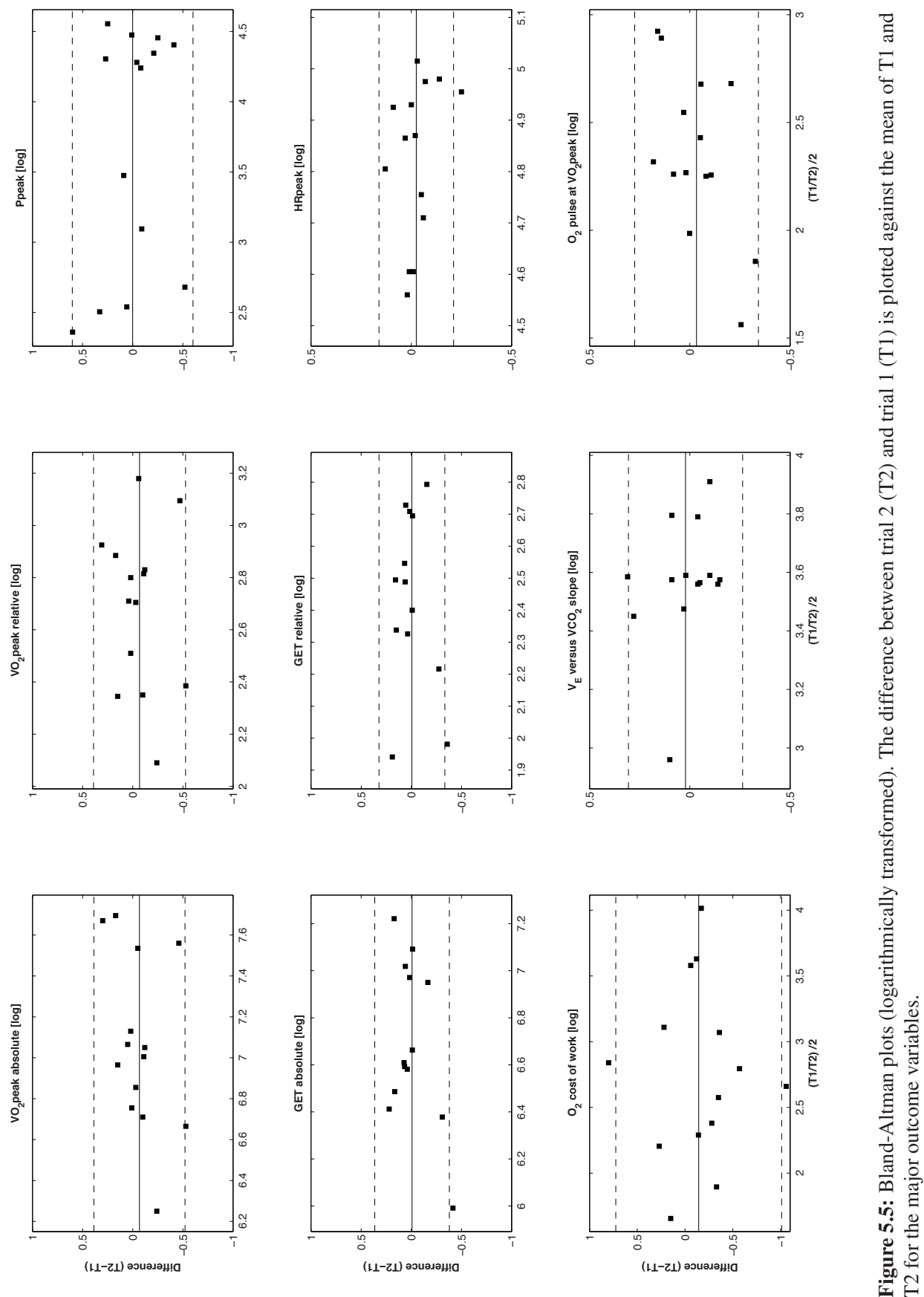

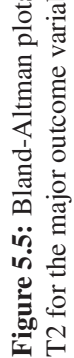

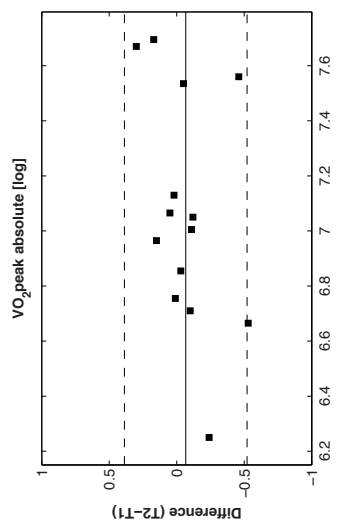




\section{Discussion}

This is the first study to evaluate the test-retest reliability and repeatability of FC-RATE for assessment of exercise capacity early after severe stroke. The aims were: (1) to assess the clinical feasibility of FC-RATE for CPET in severely motor impaired individuals early after stroke, (2) to examine the ability of the concept to meet standard cardiopulmonary criteria for maximal exercise capacity, and (3) to assess the test-retest reliability and the repeatability of the approach.

\section{General observations}

Despite rigorous exclusion criteria, only $90 \%$ of the sample completed both CLTs and $70 \%$ completed both IETs. Of the 6 subjects who dropped out during the study, only 2 were due to reasons based on uncontrollable factors such as cerebrospinal fluid leak and acute respiratory infection. The remaining 4 dropouts were caused by controllable factors such as abnormal gait pattern, tibia skin lesion, severe groin pain and lack of motivation. Skin lesions and severe groin pain due to inappropriate padding are preventable by extended familiarisation and padding procedures, whereas abnormal gait patterns due to spasticity and lack of motivation are difficult factors to control. Advanced control strategies might provide solutions for abnormal gait patterns and virtual reality approaches might facilitate motivation in the near future. Nevertheless, dropout rates were comparable with previous CPET studies in subacute stroke $[3,9,10,11]$.

The guidance of work rate for FC-RATE-based CPET was successful. RMSE $E_{P}$ values were below 10W, which can be seen as acceptable based on previous pilot study results [39]. The approach presented here used work rate values of both legs together and does not consider the severe hemiplegia of the included subjects. As a result, subjects generally tend to exercise using the unaffected side more dominantly, which led to deviations from the predefined physiological gait pattern. The powered exoskeleton allowed the subjects to remain in an acceptable movement trajectory during FC-RATE. While the approach presented here aimed to recruit as much muscle mass as possible to provoke peak exercise capacity, the imbalance of muscular activation during FC-RATE might be relevant when applying the method in longitudinal training interventions, because continuous imbalance in the gait cycle might facilitate unwanted compensation patterns.

Overall, the findings present a promising method for CPET in severely motor impaired individuals after stroke, but important factors such as appropriate padding and force interactions between subject and robot must be well controlled to gain improvements towards clinical feasibility. 


\section{Exercise capacity}

For CLT, the difficulty of estimating the time constants for $\dot{\mathrm{V}} \mathrm{O}_{2}$ uptake kinetics $(\tau)$ in the transitions from rest to passive walking and passive walking to active walking was due to the inherent noisiness of the breath-by-breath data and the consequent poor signal-to-noise ratio. The approach presented here seems not appropriate to provide consistent CLT outcome values for the transition phases. Sudden onset of changes in BWS and walking pattern seem to have a strong impact on individual performance levels during conventional RATE that restricts valid assessment of $\tau$ values. Cost of passive walking was comparable with previous studies using an identical setup [26, 27], whereas cost of active walking was considerably higher than previous results [39]. This difference might be caused by the inclusion of non-ambulatory subjects showing severe motor impairments (FAC 2.3 vs. FAC 1.1) in the present study.

With relative $\dot{\mathrm{VO}_{2}}$ peak values of $15.5 \pm 4.9 \mathrm{~mL} / \mathrm{min} / \mathrm{kg}$ ( $51 \%$ of predicted $\dot{\mathrm{V}} \mathrm{O}_{2} \mathrm{max}$ ), the results confirm that exercise capacity is seriously reduced within this group of severely motor impaired stroke survivors. Peak performance parameters during IET found in this study were slightly higher compared to previous trials using leg cycle ergometry, body weight supported treadmill training, and combined upper- and lower-limb ergometry [3, 4, 9, 10, 11, 12]. This finding might be due to the introduction of treadmill exercise based CPET that has previously confirmed higher $\dot{\mathrm{V}} \mathrm{O}_{2}$ peak values compared to leg cycle ergometry protocols [57]. Furthermore, the feedbackcontrol approach presented in this study might have recruited additional muscle mass that provoked higher peak values compared to body weight supported treadmill training. Considering the inclusion of individuals with serious motor impairments and the comparable peak cardiovascular performance parameters, this study opens new perspectives regarding assessment of exercise capacity early after severe stroke.

The GET values observed in the current study ( $\mathrm{GET} \%=72.6 \pm 12.2 \%$ of $\mathrm{VO}_{2}$ peak) were in the upper range compared to sedentary healthy individuals (50-76\% of $\mathrm{VO}_{2}$ peak) [58], providing additional evidence of compromised exercise capacity in this population. $\Delta \dot{\mathrm{V}} \mathrm{O}_{2} / \Delta \mathrm{P}$ was higher compared to leg cycle ergometry and conventional treadmill exercise meaning that subjects required more oxygen for a given work rate level; this may be explained by a substantial amount of unaccounted work performed during the test [59, 60, 61]. Further research seems indicated to explore the impact on $\Delta \dot{\mathrm{V}} \mathrm{O}_{2} / \Delta \mathrm{P}$ while walking within different robotics-assisted systems.

Although the current study provides first evidence for clinical feasibility of using FC-RATE for CPET and promising results regarding assessment of maximal exercise capacity, the issue remains of whether the concept is able to meet traditional criteria for true maximal capacity. Only 2 subjects within this study showed a plateau in $\dot{\mathrm{VO}}_{2}$ at the end of IET, traditionally considered the 
primary criterion for maximal aerobic effort. This finding is in line with previous studies [3, 10]. Even in healthy people, a plateau in $\dot{\mathrm{VO}}_{2}$ response is not always seen during IET [62], therefore this criterion must be reconsidered for future analyses. With respect to the RER, only 1 subject achieved an RER value $\geq 1.15$, and only 3 subjects ( $21 \%$ ) reached RER $\geq 1.0$. Compared to previous studies in subacute stroke which have shown mean RERpeak vales of 0.9 [4], 1.0 [3, 10], 1.02 [13], 1.1 [9], the results presented here are clearly in the lower range, but not unusual in this early phase after stroke. At least 5 subjects (36\%) reached peak heart rate within 10 beats per minute of the age-predicted heart rate maximum, which is comparable with previous findings [3, 11]. Considering these results, most of the subjects appear not to have reached their maximal aerobic capacity. The main reason might be generalised and/or leg fatigue, because $93 \%$ of the subjects terminated the IET due to inability to maintain the target work rate, suggesting that impairments in strength, coordination, and sensorimotor control contribute to difficulties in producing high work rate levels. These findings are consistent with previous studies performing CPET in subacute stroke [4, 10]. Advanced control strategies of powered exoskeletons, i.e. adapting the movement trajectory to the subjects needs (impairment level, hemiplegic side) and synchronising the treadmill inclination, might allow a more appropriate challenge progression to reach higher physical performance levels in this severely impaired population, and might provide closer approximations and comparisons to conventional treadmill based exercise testing procedures such as the Bruce or Balke protocols $[59,63]$.

Although most of the subjects appear to have performed in the submaximal range, the estimation of the work rate slope using MWC-W was shown to be successful. The approach implemented was able to reach peak performance within $8-12$ minutes $\left(\mathrm{tVO}_{2}\right.$ peak $\left.=8.2 \pm 2.6 \mathrm{~min}\right)$ during IET by defining the walking cadence at 60 steps/min while increasing the target work rate profile. This is an important finding for further research regarding the initial estimation of target work rate profiles for CPET in severely motor impaired populations.

Finally, considering the peak performance results and the low frequency of achieved criteria for $\dot{\mathrm{VO}_{2}}$ max of this study, in combination with previous study results on peak exercise capacity in subacute stroke, we hypothesise that the guidelines postulated for healthy populations may not be realistic for determination of true exercise capacity in the early stages after stroke $[64,65,66]$. 


\section{Test-retest reliability and repeatability}

Most studies that examined test-retest reliability using CPET after stroke reported excellent relative reliability, whereas only Tang et al. revealed fair to good associations between trials $[10,14,15,17$, 18]. The present study using a novel robotics-assisted treadmill-based method for assessment of exercise capacity revealed good to excellent relative reliability for the major peak cardiopulmonary performance parameters. There is only limited evidence so far on absolute reliability for CPET early after stroke. Compared to a previous study in chronic stroke using leg cycle ergometry that has shown SEM for relative $\dot{\mathrm{VO}}_{2}$ peak of $1 \mathrm{~mL} / \mathrm{min} / \mathrm{kg}(6 \%)$, our approach presented higher SEM vales (2 $\mathrm{mL} / \mathrm{min} / \mathrm{kg}(13 \%))$. Likewise, a previous study that used semi-recumbent leg cycle ergometry in subacute stroke with cognitive impairments yielded considerably lower MDC values (e.g. relative $\mathrm{VO}_{2}$ peak: $0.97 \mathrm{~mL} / \mathrm{min} / \mathrm{kg}(4 \%)$ vs. $5.6 \mathrm{~mL} / \mathrm{min} / \mathrm{kg}(36 \%)$ ) compared to our study.

While studies in healthy subjects and individuals with cardiac or respiratory disease have revealed high repeatability $(\mathrm{CoV}<0.10)$ [64, 67, 68, 69], the present study yielded considerably higher $\mathrm{CoV}$ for the major cardiopulmonary parameters (absolute $\dot{\mathrm{V}} \mathrm{O}_{2}$ peak $=0.28$, relative $\dot{\mathrm{V}} \mathrm{O}_{2}$ peak $=0.25$, Ppeak $=0.28$, HRpeak $=0.10$, absolute $\mathrm{GET}=0.14$, relative $\mathrm{GET}=0.14$, $\Delta \dot{\mathrm{V}} \mathrm{O}_{2} / \Delta \mathrm{P}=0.36, \mathrm{O}_{2}$ pulse $\left.=0.17, \Delta \dot{\mathrm{V}}_{\mathrm{E}} / \Delta \dot{\mathrm{V} C O}_{2}=0.14\right)$.

There was visual suspicion of heteroscedasticity for absolute $\dot{\mathrm{V}}_{2}$ peak and Ppeak; however, logarithmic transformation of the data did not change the relevant outcome variables, and thus appeared irrelevant fur further consideration. We hypothesise that the increase in bias along with higher work rate values is caused by large day-to-day variability in strength and/or coordinative capabilities. Although general factors that may contribute to variability in test-retest situations such as disease severity, patient instruction, time of day, testing procedure, and equipment, were well controlled in the protocol presented here. However, the novel approach might have led to additional confounding factors that could have influenced test-retest reliability and repeatability. A major factor was the high coordinative demand of the concept. Subjects not only had to walk (or pedal, as in earlier cycle ergometry studies); the challenge was to produce additional forces in the walking direction, where the exoskeleton restricted the movement. The results clearly indicated that the major reason for test termination was the inability to maintain $\mathrm{P}_{\text {mech }}^{*}$, which led to the assumption that muscular and/or coordinative fatigue was the reason for test termination. Therefore, variation might be reinforced by day-to-day variability (normally 3\% [64]) and influenced by whether the test was maximal or not. This hypothesis is supported by the low RERpeak values reported in this study. More sophisticated strategies are required to reduce the load on the neuromuscular system while increasing cardiovascular stress during FC-RATE. This will possibly lead to a better 
approximation of true exercise capacity early after stroke and might improve the reliability and the repeatability of FC-RATE-based CPET.

The approach presented here seems suitable for comparison of groups of stroke individuals or for assessment of group intervention effects in future studies, considering the range of betweengroup improvement in $\dot{\mathrm{VO}_{2}}$ peak of 12.6-34.8\% [70, 71, 72, 73] and Ppeak of 23.4-176.9\% [72, $73,74]$ after cardiovascular exercise in subacute stroke. Whether the absolute reliability and the repeatability reported are adequate to identify effectiveness of intervention programmes to improve exercise capacity should be part of future studies including larger sample sizes.

\section{Limitations}

The major limitation of the current study is the small sample size, which may render the results underpowered. A sample size of at least 50 is generally seen as adequate for the assessment of the agreement parameter, based on a general guideline by Altman [75]. Considering the experimental approach of the method and the difficulty of implementing and performing CPET in the early stages after severe stroke, our sample of 20 subjects at onset was a realistic group size to evaluate first estimates from a clinical perspective.

The conventional sequencing of the test situations (CLT, CLT, IET, IET) might have led to practice effects. The severely impaired and early-post-stroke status of the individuals included in this experimental approach may justify this order to progressively increase the exercise intensity over time to control potential risks.

The present study protocol did not include ECG monitoring for reasons of practicability, which influenced the study sample by excluding individuals with cardiac risk factors for CPET. While around $75 \%$ of stroke survivors present some degree of cardiovascular disease [76], the sample of this study might not be representative. Nevertheless, there was an uncontrolled risk for cardiac events due to the absence of ECG despite the adherence to strict exclusion criteria for cardiovascular disease.

While the study protocol strictly controlled time of day for CPET, the tests were performed within $48 \mathrm{~h}$ or $72 \mathrm{~h}$ due to practical reasons. This time difference might have affected the recovery phase of the subjects, thus influencing the results. 


\section{Conclusion}

This study presents first evidence on reliability and repeatability for CPET in severely motor impaired individuals early after stroke using a feedback-controlled robotics-assisted treadmill. The results demonstrate good to excellent test-retest reliability and appropriate repeatability for the most important peak cardiopulmonary performance parameters. These findings have important implications for the design and implementation of cardiovascular exercise interventions in severely impaired populations. Future research needs to develop advanced control strategies to enable the true limit of functional exercise capacity to be reached and to further assess test-retest reliability and repeatability in larger samples.

\section{References}

[1] J. Myers, M. Prakash, V. Froelicher, D. Do, S. Partington, and J. E. Atwood, "Exercise capacity and mortality among men referred for exercise testing," New England Journal of Medicine, vol. 346, no. 11, pp. 793-801, 2002 .

[2] J. Paffenbarger, R. S., R. T. Hyde, A. L. Wing, I. M. Lee, D. L. Jung, and J. B. Kampert, "The association of changes in physical-activity level and other lifestyle characteristics with mortality among men," $N$ Engl J Med, vol. 328 , no. 8 , pp. $538-45,1993$.

[3] M. J. MacKay-Lyons and L. Makrides, "Exercise capacity early after stroke," Archives of Physical Medicine and Rehabilitation, vol. 83, no. 12, pp. 1697-1702, 2002.

[4] J. O. Kelly, S. L. Kilbreath, G. M. Davis, B. Zeman, and J. Raymond, "Cardiorespiratory fitness and walking ability in subacute stroke patients," Archives of Physical Medicine Rehabilitation, vol. 84, no. 12, pp. 1780$1785,2003$.

[5] M. J. MacKay-Lyons and L. Makrides, "Longitudinal changes in exercise capacity after stroke," Archives of Physical Medicine and Rehabilitation, vol. 85, no. 10, pp. 1608-1612, 2004.

[6] E. J. Roth, "Heart disease in patients with stroke. part 2: Impact and implications for rehabilitation," Archives of Physical Medicine and Rehabilitation, vol. 75, no. 1, pp. 94-101, 1994.

[7] D. H. Saunders, C. A. Greig, A. Young, and G. E. Mead, "Physical fitness training for patients with stroke an updated review," Stroke, vol. 41, no. 3, pp. E160-E161, 2010.

[8] S. A. Billinger, R. Arena, J. Bernhardt, J. J. Eng, B. A. Franklin, C. M. Johnson, M. MacKay-Lyons, R. F. Macko, G. E. Mead, E. J. Roth, M. Shaughnessy, A. Tang, C. American Heart Association Stroke, C. Council on, N. Stroke, L. Council on, H. Cardiometabolic, E. Council on, Prevention, and C. Council on Clinical, "Physical activity and exercise recommendations for stroke survivors: a statement for healthcare professionals from the american heart association/american stroke association," Stroke, vol. 45, no. 8, pp. 2532-53, 2014.

[9] J. K. Chen, T. W. Chen, C. H. Chen, and M. H. Huang, "Preliminary study of exercise capacity in post-acute stroke survivors," Kaohsiung Journal of Medical Sciences, vol. 26, no. 4, pp. 175-181, 2010.

[10] A. Tang, K. M. Sibley, S. G. Thomas, W. E. McIlroy, and D. Brooks, "Maximal exercise test results in subacute stroke," Archives of Physical Medicine and Rehabilitation, vol. 87, no. 8, pp. 1100-1105, 2006. 
[11] J. S. Yates, S. Studenski, S. Gollub, R. Whitman, S. Perera, S. M. Lai, and P. W. Duncan, "Bicycle ergometry in subacute-stroke survivors: Feasibility, safety, and exercise performance," Journal of Aging and Physical Activity, vol. 12, no. 1, pp. 64-74, 2004.

[12] D. C. Hill, K. A. Ethans, D. A. MacLeod, E. R. Harrison, and J. E. Matheson, "Exercise stress testing in subacute stroke patients using a combined upper- and lower-limb ergometer," Archives of Physical Medicine and Rehabilitation, vol. 86, no. 9, pp. 1860-1866, 2005.

[13] I. Baert, D. Daly, E. Dejaeger, C. Vanroy, Y. Vanlandewijck, and H. Feys, "Evolution of cardiorespiratory fitness after stroke: A 1-year follow-up study. influence of pre-stroke patients' characteristics and stroke-related factors," Cerebrovascular Diseases, vol. 31, p. 189, 2011.

[14] C. L. Dobrovolny, F. M. Ivey, M. A. Rogers, J. D. Sorkin, and R. F. Macko, "Reliability of treadmill exercise testing in older patients with chronic hemiparetic stroke," Archives of Physical Medicine Rehabilitation, vol. 84, no. 9, pp. 1308-12, 2003.

[15] J. J. Eng, A. S. Dawson, and K. S. Chu, "Submaximal exercise in persons with stroke: Test-retest reliability and concurrent validity with maximal oxygen consumption," Archives of Physical Medicine and Rehabilitation, vol. 85, no. 1, pp. 113-118, 2004.

[16] R. F. Macko, L. I. Katzel, A. Yataco, L. D. Tretter, C. A. DeSouza, D. R. Dengel, G. V. Smith, and K. H. Silver, "Low-velocity graded treadmill stress testing in hemiparetic stroke patients," Stroke, vol. 28, no. 5, pp. 988-992, 1997.

[17] O. C. Lennon, R. S. Denis, N. Grace, and C. Blake, "Feasibility, criterion validity and retest reliability of exercise testing using the astrand-rhyming test protocol with an adaptive ergometer in stroke patients," Disabil Rehabil, vol. 34 , no. 14 , pp. 1149-56, 2012.

[18] C. Olivier, J. Dore, S. Blanchet, D. Brooks, C. L. Richards, G. Martel, N. M. Robitaille, and D. B. Maltais, "Maximal cardiorespiratory fitness testing in individuals with chronic stroke with cognitive impairment: practice test effects and test-retest reliability," Arch Phys Med Rehabil, vol. 94, no. 11, pp. 2277-82, 2013.

[19] S. A. Billinger, B. Y. Tseng, and P. M. Kluding, "Modified total-body recumbent stepper exercise test for assessing peak oxygen consumption in people with chronic stroke," Physical Therapy, vol. 88, no. 10, pp. 1188-95, 2008 .

[20] L. J. Durstine, G. Moore, P. Painter, and S. Roberts, ACSM's Exercise Management for Persons with Chronic Diseases and Disabilities. Champaign, IL: Human Kinetics, 3rd ed., 2009.

[21] B. French, L. H. Thomas, M. J. Leathley, C. J. Sutton, J. McAdam, A. Forster, P. Langhorne, C. I. M. Price, A. Walker, and C. L. Watkins, "Repetitive task training for improving functional ability after stroke," Cochrane Database of Systematic Reviews, no. 4, p. CD006073, 2007.

[22] P. Langhorne, F. Coupar, and A. Pollock, "Motor recovery after stroke: a systematic review," Lancet Neurology, vol. 8, no. 8, pp. 741-754, 2009.

[23] M. Mackay-Lyons, A. McDonald, J. Matheson, G. Eskes, and M. A. Klus, "Dual effects of body-weight supported treadmill training on cardiovascular fitness and walking ability early after stroke: a randomized controlled trial," Neurorehabil Neural Repair, vol. 27, no. 7, pp. 644-53, 2013.

[24] S. Hesse, C. Bertelt, M. T. Jahnke, A. Schaffrin, P. Baake, M. Malezic, and K. H. Mauritz, "Treadmill training with partial body weight support compared with physiotherapy in nonambulatory hemiparetic patients," Stroke, vol. 26, no. 6, pp. 976-81, 1995.

[25] A. Danielsson and K. S. Sunnerhagen, "Oxygen consumption during treadmill walking with and without body weight support in patients with hemiparesis after stroke and in healthy subjects," Archives of Physical Medicine Rehabilitation, vol. 81, no. 7, pp. 953-957, 2000. 
[26] M. MacKay, L. Makrides, and S. Speth, "Effect of 15exercise capacity of adults without impairments," Physical Therapy, vol. 81, no. 11, pp. 1790-1800, 2001.

[27] C. Krewer, F. Müller, B. Husemann, S. Heller, J. Quintern, and E. Koenig, "The influence of different lokomat walking conditions on the energy expenditure of hemiparetic patients and healthy subjects," Gait Posture, vol. 26, no. 3, pp. 372-377, 2007.

[28] O. Stoller, E. D. de Bruin, M. Schindelholz, C. Schuster, R. A. de Bie, and K. J. Hunt, "Evaluation of exercise capacity after severe stroke using robotics-assisted treadmill exercise: A proof-of-concept study," Technology and Health Care, vol. 21, no. 2, pp. 157-166, 2013.

[29] T. G. Hornby, D. D. Campbell, D. H. Zemon, and J. H. Kahn, "Clinical and quantitative evaluation of roboticassisted treadmill walking to retrain ambulation after spinal cord injury," Topics in Spinal Cord Injury Rehabilitation, vol. 11, no. 2, pp. 1-17, 2005.

[30] M. S. Nash, P. L. Jacobs, B. M. Johnson, and E. Field-Fote, "Metabolic and cardiac responses to robotic-assisted locomotion in motor-complete tetraplegia: A case report," Journal of Spinal Cord Medicine, vol. 27, no. 1, pp. 78-82, 2004.

[31] M. P. van Nunen, K. H. Gerrits, A. de Haan, and T. W. Janssen, "Exercise intensity of robot-assisted walking versus overground walking in nonambulatory stroke patients," J Rehabil Res Dev, vol. 49, no. 10, pp. 1537-46, 2012 .

[32] J. F. Israel, D. D. Campbell, J. H. Kahn, and T. G. Hornby, "Metabolic costs and muscle activity patterns during robotic- and therapist-assisted treadmill walking in individuals with incomplete spinal cord injury," Physical Therapy, vol. 86, no. 11, pp. 1466-1478, 2006.

[33] K. J. Hunt, L. P. Jack, A. Pennycott, C. Perret, M. Baumberger, and T. H. Kakebeeke, "Control of work ratedriven exercise facilitates cardiopulmonary training and assessment during robot-assisted gait in incomplete spinal cord injury," Biomedical Signal Processing and Control, vol. 3, no. 1, pp. 19-28, 2008.

[34] A. Koenig, X. Omlin, J. Bergmann, L. Zimmerli, M. Bolliger, F. Muller, and R. Riener, "Controlling patient participation during robot-assisted gait training," J Neuroeng Rehabil, vol. 8, p. 14, 2011.

[35] A. Pennycott, K. J. Hunt, L. P. Jack, C. Perret, and T. H. Kakebeeke, "Estimation and volitional feedback control of active work rate during robot-assisted gait," Control Engineering Practice, vol. 17, no. 2, pp. 322-328, 2008.

[36] T. Lam, K. Pauhl, A. Krassioukov, and J. J. Eng, "Using robot-applied resistance to augment body-weightsupported treadmill training in an individual with incomplete spinal cord injury," Phys Ther, vol. 91, no. 1, pp. 143-51, 2011.

[37] A. Duschau-Wicke, A. Caprez, and R. Riener, "Patient-cooperative control increases active participation of individuals with sci during robot-aided gait training," Journal of Neuroengineering and Rehabilitation, vol. 7 , p. $43,2010$.

[38] R. Banz, M. Bolliger, S. Muller, C. Santelli, and R. Riener, "A method of estimating the degree of active participation during stepping in a driven gait orthosis based on actuator force profile matching," IEEE Trans Neural Syst Rehabil Eng, vol. 17, no. 1, pp. 15-22, 2009.

[39] O. Stoller, M. Schindelholz, L. Bichsel, C. Schuster, R. A. de Bie, E. D. de Bruin, and K. J. Hunt, "Feedbackcontrolled robotics-assisted treadmill exercise to assess and influence aerobic capacity early after stroke: a proofof-concept study," Disabil Rehabil Assist Technol, vol. 9, no. 4, pp. 271-278, 2014.

[40] W. R. Thompson, N. F. Gordon, and L. S. Pescatello, American College of Sports Medicine. Guidelines for Exercise Testing and Prescription. Philadelphia: Lippincott Williams Wilkins, eight ed., 2010.

[41] F. M. Collen, D. T. Wade, and C. M. Bradshaw, "Mobility after stroke: reliability of measures of impairment and disability," Int Disabil Stud, vol. 12, no. 1, pp. 6-9, 1990. 
[42] C. Collin, D. T. Wade, S. Davies, and V. Horne, "The barthel adl index: a reliability study," Int Disabil Stud, vol. 10, no. 2, pp. 61-3, 1988.

[43] J. Perry, Gait analysis: normal and pathological function. Thorofare, NJ: SLACK incorporated, 1992.

[44] H. Tanaka, K. D. Monahan, and D. R. Seals, "Age-predicted maximal heart rate revisited," Journal of the American College of Cardiology, vol. 37, no. 1, pp. 153-156, 2001.

[45] P. A. Tesch, "Exercise performance and beta-blockade," Sports Medicine, vol. 2, no. 6, pp. 389-412, 1985.

[46] G. A. Borg, "Perceived exertion,” Exerc Sport Sci Rev, vol. 2, pp. 131-153, 1974.

[47] M. Schindelholz, O. Stoller, and K. J. Hunt, "A software module for cardiovascular rehabilitation in roboticsassisted treadmill exercise,” Biomedical Signal Processing and Control, vol. 10, pp. 296-307, 2014.

[48] R. A. Robergs, D. Dwyer, and T. Astorino, "Recommendations for improved data processing from expired gas analysis indirect calorimetry," Sports Medicine, vol. 40, no. 2, pp. 95-111, 2010.

[49] B. J. Whipp and K. Wasserman, “Oxygen-uptake kinetics for various intensities of constant-load work,” Journal of Applied Physiology, vol. 33, no. 3, pp. 351-356, 1972.

[50] D. C. Poole, D. P. Wilkerson, and A. M. Jones, "Validity of criteria for establishing maximal o2 uptake during ramp exercise tests," Eur J Appl Physiol, vol. 102, no. 4, pp. 403-10, 2008.

[51] W. L. Beaver, K. Wasserman, and B. J. Whipp, "A new method for detecting anaerobic threshold by gasexchange," Journal of Applied Physiology, vol. 60, no. 6, pp. 2020-2027, 1986.

[52] D. L. Streiner, "Diagnosing tests: using and misusing diagnostic and screening tests," J Pers Assess, vol. 81, no. 3, pp. 209-19, 2003.

[53] D. L. Streiner and G. R. Norman, Health measurement scales: a practical guide to their development and use. Oxford, NY: Oxford university press, 2008.

[54] J. P. Weir, "Quantifying test-retest reliability using the intraclass correlation coefficient and the sem," J Strength Cond Res, vol. 19, no. 1, pp. 231-40, 2005.

[55] S. M. Haley and M. A. Fragala-Pinkham, "Interpreting change scores of tests and measures used in physical therapy," Physical Therapy, vol. 86, no. 5, pp. 735-743, 2006.

[56] R. Jurca, A. S. Jackson, M. J. LaMonte, J. R. Morrow, S. N. Blair, N. J. Wareham, W. L. Haskell, W. van Mechelen, T. S. Church, J. M. Jakicic, and R. Laukkanen, "Assessing cardiorespiratory fitness without performing exercise testing," American Journal of Preventive Medicine, vol. 29, no. 3, pp. 185-193, 2005.

[57] G. A. McKay and E. W. Banister, "A comparison of maximum oxygen uptake determination by bicycle ergometry at various pedaling frequencies and by treadmill running at various speeds," Eur J Appl Physiol Occup Physiol, vol. 35, no. 3, pp. 191-200, 1976.

[58] J. A. Davis, T. W. Storer, and V. J. Caiozzo, "Prediction of normal values for lactate threshold estimated by gas exchange in men and women," Eur J Appl Physiol Occup Physiol, vol. 76, no. 2, pp. 157-64, 1997.

[59] K. Wasserman, J. E. Hansen, D. Y. Sue, W. W. Stringer, K. E. Sietsema, X. G. Sun, and B. J. Whipp, Principles of exercise testing and interpretation: including pathophysiology and clinical applications. Philadelphia, PA: Lippincott Williams Wilkins, 2011.

[60] J. E. Hansen, R. Casaburi, D. M. Cooper, and K. Wasserman, "Oxygen uptake as related to work rate increment during cycle ergometer exercise,” Eur J Appl Physiol Occup Physiol, vol. 57, no. 2, pp. 140-5, 1988. 
[61] J. Porszasz, R. Casaburi, A. Somfay, L. J. Woodhouse, and B. J. Whipp, "A treadmill ramp protocol using simultaneous changes in speed and grade," Medicine and Science in Sports and Exercise, vol. 35, no. 9, pp. 1596$1603,2003$.

[62] J. R. Day, H. B. Rossiter, E. M. Coats, A. Skasick, and B. J. Whipp, "The maximally attainable vo2 during exercise in humans: the peak vs. maximum issue," Journal of applied physiology, vol. 95, no. 5, pp. 1901-1907, 2003.

[63] R. A. Bruce, M. N. Cooper, G. O. Gey, L. D. Fisher, and D. R. Peterson, "Variations in responses to maximal exercise in health and in cardiovascular disease," Angiology, vol. 24, no. 11, pp. 691-702, 1973.

[64] "Ats/accp statement on cardiopulmonary exercise testing," American Journal of Respiratory and Critical Care Medicine, vol. 167, pp. 211-277, 2003.

[65] G. J. Balady, R. Arena, K. Sietsema, J. Myers, L. Coke, G. F. Fletcher, D. Forman, B. Franklin, M. Guazzi, M. Gulati, S. J. Keteyian, C. J. Lavie, R. MacKo, D. Mancini, and R. V. Milani, "Clinician's guide to cardiopulmonary exercise testing in adults: A scientific statement from the american heart association," Circulation, vol. 122 , no. 2 , pp. 191-225, 2010.

[66] G. F. Fletcher, G. J. Balady, E. A. Amsterdam, B. Chaitman, R. Eckel, J. Fleg, V. F. Froelicher, A. S. Leon, I. L. Pina, R. Rodney, D. G. Simons-Morton, M. A. Williams, and T. Bazzarre, "Exercise standards for testing and training - a statement for healthcare professionals from the american heart association," Circulation, vol. 104, no. 14, pp. 1694-1740, 2001.

[67] C. S. Garrard and C. Emmons, "The reproducibility of the respiratory responses to maximum exercise," Respiration, vol. 49, no. 2, pp. 94-100, 1986.

[68] J. E. Nordrehaug, R. Danielsen, L. Stangeland, G. A. Rosland, and H. Vik-Mo, "Respiratory gas exchange during treadmill exercise testing: reproducibility and comparison of different exercise protocols," Scand J Clin Lab Invest, vol. 51, no. 7, pp. 655-8, 1991.

[69] A. Barron, N. Dhutia, J. Mayet, A. D. Hughes, D. P. Francis, and R. Wensel, "Test-retest repeatability of cardiopulmonary exercise test variables in patients with cardiac or respiratory disease," Eur J Prev Cardiol, vol. 21, no. 4 , pp. $445-53,2014$.

[70] I. T. da Cunha, P. A. Lim, H. Qureshy, H. Henson, T. Monga, and E. J. Protas, "Gait outcomes after acute stroke rehabilitation with supported treadmill ambulation training: A randomized controlled pilot study," Archives of Physical Medicine and Rehabilitation, vol. 83, no. 9, pp. 1258-1265, 2002.

[71] P. Duncan, S. Studenski, L. Richards, S. Gollub, S. M. Lai, D. Reker, S. Perera, J. Yates, V. Koch, S. Rigler, and D. Johnson, "Randomized clinical trial of therapeutic exercise in subacute stroke," Stroke, vol. 34, no. 9, pp. 2173-2180, 2003.

[72] A. Letombe, C. Cornille, H. Delahaye, A. Khaled, O. Morice, A. Tomaszewski, and N. Olivier, "Early poststroke physical conditioning in hemiplegic patients: A preliminary study," Annals of Physical Rehabilitation Medicine, vol. 53, no. 10, pp. 632-642, 2010.

[73] A. Tang, K. M. Sibley, S. G. Thomas, M. T. Bayley, D. Richardson, W. E. McIlroy, and D. Brooks, "Effects of an aerobic exercise program on aerobic capacity, spatiotemporal gait parameters, and functional capacity in subacute stroke," Neurorehabilitation and Neural Repair, vol. 23, no. 4, pp. 398-406, 2009.

[74] M. Katz-Leurer, M. Shochina, E. Carmeli, and Y. Friedlander, "The influence of early aerobic training on the functional capacity in patients with cerebrovascular accident at the subacute stage," Archives of Physical Medicine and Rehabilitation, vol. 84, no. 11, pp. 1609-1614, 2003.

[75] D. G. Altman, Practical statistics for medical research. CRC Press, 1990.

[76] E. J. Roth, "Heart disease in patients with stroke: incidence, impact, and implications for rehabilitation. part 1: Classification and prevalence," Archives of Physical Medicine and Rehabilitation, vol. 74, no. 7, pp. 752-760, 1993. 


\section{Chapter 6}

\section{Cardiovascular rehabilitation soon after stroke using feedback-controlled}

robotics-assisted treadmill exercise: study protocol of a randomised controlled pilot trial

Trials 2013;14:304

doi: 10.1186/1745-6215-14-304

Oliver Stoller Eling D de Bruin Corina Schuster-Amft Matthias Schindelholz Rob A de Bie Kenneth J Hunt 


\section{Abstract}

Background: After experiencing a stroke, most individuals also suffer from cardiac disease, are immobile and thus have low endurance for exercise. Aerobic capacity is seriously reduced in these individuals and does not reach reasonable levels after conventional rehabilitation programmes. Cardiovascular exercise is beneficial for improvement of aerobic capacity in mild to moderate stroke. However, less is known about its impact on aerobic capacity, motor recovery, and quality of life in severely impaired individuals. The aim of this pilot study is to explore the clinical efficacy and feasibility of cardiovascular exercise with regard to aerobic capacity, motor recovery, and quality of life using feedback-controlled robotics-assisted treadmill exercise in non-ambulatory individuals soon after experiencing a stroke.

Methods/Design: This will be a single-centred single blind, randomised control trial with a prepost intervention design. Subjects will be recruited early after their first stroke ( $\leq 20$ weeks) at a neurological rehabilitation clinic and will be randomly allocated to an inpatient cardiovascular exercise programme that uses feedback-controlled robotics-assisted treadmill exercise (experimental) or to conventional robotics-assisted treadmill exercise (control). Intervention duration depends on the duration of each subject's inpatient rehabilitation period. Aerobic capacity, as the primary outcome measure, will be assessed using feedback-controlled robotics-assisted treadmill-based cardiopulmonary exercise testing. Secondary outcome measures will include gait speed, walking endurance, standing function, and quality of life. Outcome assessment will be conducted at baseline, after each 4-week intervention period, and before clinical discharge. Ethical approval has been obtained.

Discussion: Whether cardiovascular exercise in non-ambulatory individuals early after stroke has an impact on aerobic capacity, motor recovery, and quality of life is not yet known. Feedbackcontrolled robotics-assisted treadmill exercise is a relatively recent intervention method and might be used to train and evaluate aerobic capacity in this population. The present pilot trial is expected to provide new insights into the implementation of early cardiovascular exercise for individuals with severe motor impairment. The findings of this study may guide future research to explore the effects of early cardiovascular activation after severe neurological events.

Trial registration: This trial is registered with the Clinical Trials.gov Registry (NCT01679600). 


\section{Background}

Cardiovascular-related diseases are the leading causes of death [1] and long-term disability worldwide [2]. Stroke affects about 15 million people each year, whereby 5 million die and another 5 million remain permanently disabled [3]. It has been estimated that stroke will occur in $35 \%$ of the population over the age of 65 , a group that will increase in proportion due to demographic shifts in most populations [4]. This leads to an increased need for effective rehabilitation programmes to enhance recovery, improve functional status and quality of life, while considering the future challenges in worldwide health care economics [5].

To date, stroke rehabilitation research has focussed primarily on restoring motor control to promote independent function during daily life. However, around $75 \%$ of post-stroke individuals suffer from cardiac disease [6,7], and the majority have low endurance for exercise as a consequence of immobility [8]. Previous work has shown that peak oxygen uptake $\left(\mathrm{V}_{2}\right.$ peak $)$ is reduced to 10 to $17 \mathrm{~mL} / \mathrm{min} / \mathrm{kg}$ within the first 30 days after stroke $[9,10,11]$ and does not rise over $20 \mathrm{~mL} / \mathrm{min} / \mathrm{kg}$ after 6 months $[11,12,13,14]$. These values are 25 to $45 \%$ lower than $\dot{\mathrm{V}} \mathrm{O}_{2}$ peak in age-matched healthy subjects $[15,16]$. This early and persistent decline in aerobic capacity can delay or inhibit participation in a therapeutic exercise programme, cause difficulties in the rehabilitation process and long-term post-stroke course of care, and limits an individuals independent performance of functional activities [7, 17].

In a recent meta-analysis it has been shown that cardiovascular exercise is beneficial for improving $\dot{\mathrm{VO}_{2}}$ peak and walking endurance in mild to moderately impaired individuals with subacute stroke (1 week to 6 months after onset) [18]. However, less is known about its impact on motor recovery, quality of life, and mortality. Previous trials did not consider post-stroke individuals with severe motor impairments due to the challenge of selecting appropriate training modalities and evaluation of effectiveness (for example, aerobic capacity). About $50 \%$ of stroke survivors may be non-ambulatory or even unable to walk a speed or distance necessary to achieve aerobic benefits [19]. This leads to inadequate training programmes and restricts the performance of valid cardiopulmonary exercise testing protocols. Given the fact that most motor recovery occurs in the first 3 months after a stroke [20], subacute rehabilitation programmes have to target neuromuscular recovery as well as cardiovascular exercise to optimise intervention outcomes. This could be achieved by the implementation of assistive devices to promote functional activities such as walking or stair climbing.

The application of robotics-assisted walking thus has substantial clinical potential. The technology offers several advantages over conventional rehabilitation strategies, such as motor func- 
tion related training programmes within controlled evaluation settings, even for individuals with severe motor impairments. Robotics-assisted treadmill exercise (RATE), consisting of driven gait orthoses with an integrated body weight unloading system, opens new perspectives for cardiovascular exercise and evaluation of aerobic capacity in the early stages after stroke.

A novel assessment protocol has been developed to estimate key cardiopulmonary performance parameters during RATE [21, 22]. This incorporates a human-in-the-loop feedback mechanism, which allows individuals to maximise their voluntary input and associated cardiovascular stress. A previous study provided evidence for the concept of feedback-controlled RATE (FCRATE) to evaluate aerobic capacity and guide cardiovascular exercise in non-ambulatory individuals with subacute stroke [23]. Cardiopulmonary performance parameters yielded reasonable values following standardised exercise testing protocols. The next logical step is to evaluate the clinical efficacy and feasibility of the concept during early stroke rehabilitation. It has been hypothesised that FC-RATE is feasible and effective for maintenance or improvement of aerobic capacity in non-ambulatory individuals with subacute stroke. This might be highly relevant for further research to explore the association between cardiovascular rehabilitation and neural plasticity during early stroke rehabilitation.

This randomised controlled pilot trial aims: (1) to explore the clinical efficacy of early cardiovascular exercise on aerobic capacity, motor recovery, and quality of life; (2) to evaluate the feasibility of FC-RATE for cardiovascular rehabilitation in non-ambulatory individuals after stroke; and (3) to put forward recommendations for development and design of larger clinical trials regarding cardiovascular rehabilitation early after stroke.

\section{Methods/Design}

All procedures involved in this trial will be conducted in compliance with national ethical standards and the Helsinki Declaration. Ethical approval has been obtained from the ethics committee of the canton of Aargau in Switzerland (Reference No. 2012/051).

\section{Design and setting}

This study is a single-centred single blind, randomised control trial with a pre-post intervention design. Subjects will be recruited at a neurological rehabilitation clinic in the northwestern part of Switzerland (Reha Rheinfelden). Figure 6.1 illustrates the plan of the study protocol. 


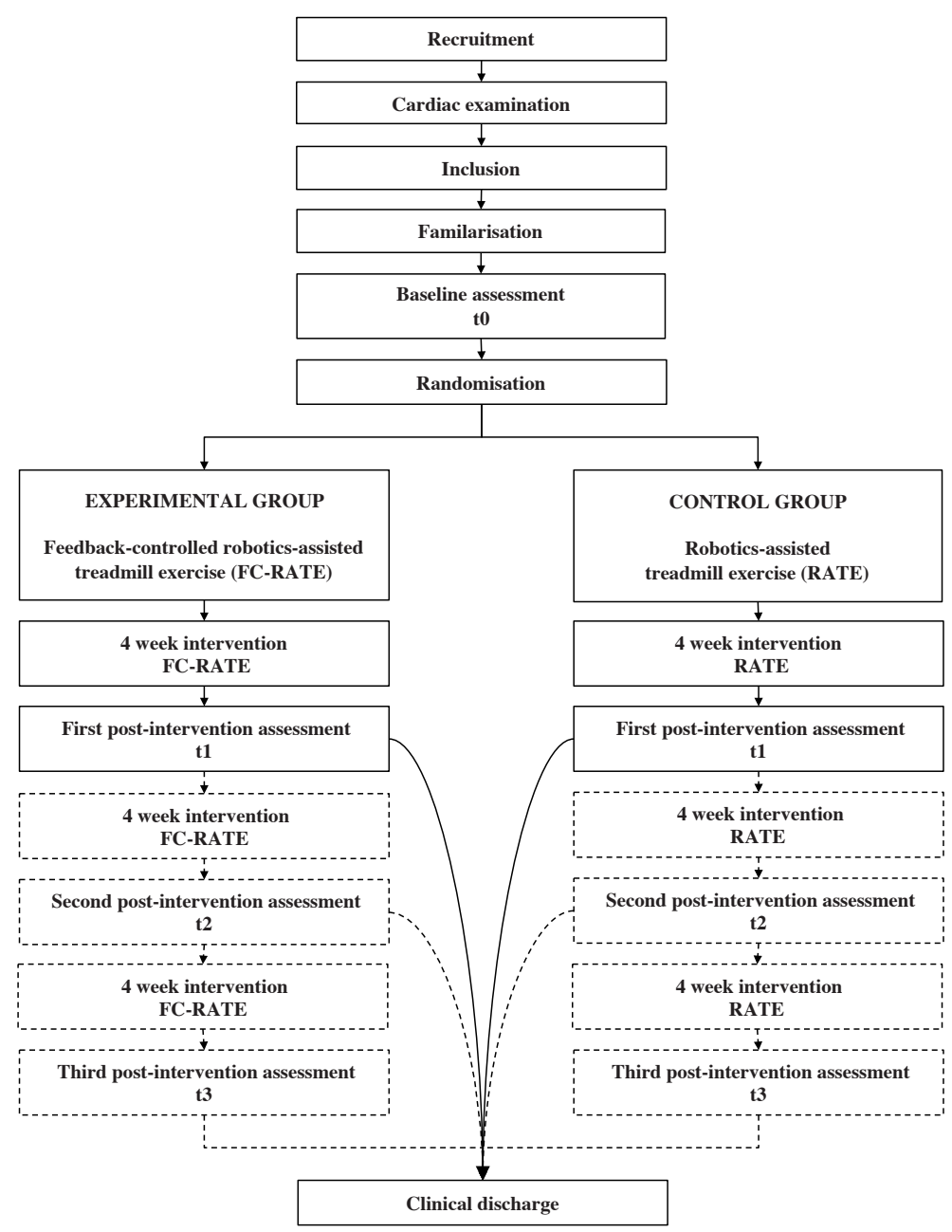

Figure 6.1: Flow chart outlining the study protocol. A 4-week intervention period includes baseline assessments (t0) and first post-intervention (t1) assessments (solid line). The dashed line represents a potential continuation of the intervention due to extended inpatient rehabilitation. Post-intervention assessments will be conducted after every 4-week intervention period and before clinical discharge. 


\section{Eligibility criteria}

Subjects will be enrolled after their first stroke if they are non-ambulatory. Inclusion criteria are: (1) clinical diagnosis of an initial stroke, (2) $\leq 20$ weeks post-stroke at intervention onset, (3) age $\geq 18$ years, (4) Functional Ambulation Classification (FAC) $\leq 3$, and (5) ability to understand the procedures and provide informed consent. Exclusion criteria are: (1) contraindications for cardiopulmonary exercise testing as outlined by the American College of Sports Medicine (ACSM) [24], (2) contraindications for RATE according to the device manufacturer, (3) concurrent neurological disease, (4) concurrent pulmonary disease, and (4) history of dementia.

\section{Recruitment}

Potential subjects will be screened daily by searching the admission lists of the rehabilitation centre. The project leader will approach eligible subjects and explain the purpose of the study and ask whether potentially qualifying individuals have an interest in participating. If a potential subject expresses interest, the study will be explained in detail, all questions will be addressed and the subject will be invited to participate by providing signed informed consent. Subjects then will be referred for specific cardiac examination by a cardiologist to detect potential contraindications for cardiopulmonary exercise testing. The project leader and the responsible physician will make a final decision on inclusion.

\section{Randomisation, allocation concealment, and blinding}

Subjects will be randomly allocated to an experimental group (FC-RATE) or to a control group (RATE). An independent researcher will generate a 4-block randomisation list and send it to the clinic's pharmacy for safekeeping. The leader of the physical therapy team will call the pharmacy to request a subjects allocation. Only the treating therapists will have access to the allocated documents. The project leader, who is blind for allocation of the subjects, will carry out all testing procedures as an independent examiner. A further research assistant will be responsible for data input. Subjects will be told not to talk to the examiner about the group allocation or therapy content during the post-intervention assessments. Randomisation will be concealed to the independent examiner until the last post-intervention assessment has been performed. The data will be analysed by the project leader. 


\section{Outcome measures}

All primary and secondary outcome measurements will be carried out at baseline (t0), after each 4week intervention period ( 1 1, t2, t3, etcetera), and before clinical discharge. At baseline, descriptive variables for each subject include age, sex, area and type of infarct, side of stroke, time since stroke onset, body height and body mass.

A summary of the primary and secondary outcome measures can be found in Table 6.1. The primary outcome will be aerobic capacity, the accepted criterion for exercise capacity [24]. We will estimate standardised cardiopulmonary performance parameters based on international guidelines [25]. Secondary outcome measures will focus on physical performance, stroke impact, and feasibility. We will evaluate the work rate tracking error during FC-RATE by calculating the root mean square error (RMSE) between actual work rate and target work rate values. Gait speed will be assessed using the 10 Meter Walk Test (10MWT) [26, 27]. Walking endurance will be measured using the 6 Minute Walk Test (6MWT), the distance that an individual is able to walk in 6 minutes on a hard, flat surface $[28,29]$. To assess basic standing function in non-ambulatory subjects, we will employ a standardised standing test on a force plate. The subject will be requested to: (1) stand in bipedal stance, (2) shift his/her body weight from the right to the left leg alternately, and (3) shift his/her body weight in a defined rhythm from the right to the left leg alternately. The test-retest reliability of centre of pressure measures in bipedal tasks has been shown previously [30]. We will use the Stroke Impact Scale (SIS) to assess recovery and quality of life after stroke [31].

The feasibility of the study protocol will be based on defined criteria for success such as compliance, attrition, safety, and successful data processing. We define feasibility to be affirmed with compliance to the study protocol of $\geq 90 \%$, an attrition rate of $\leq 10 \%$, clinical safety of $100 \%$, and effective data processing of $100 \%$. The overall feasibility of the study will be rated using the following outcomes: (i) protocol deemed feasible (when all criteria for success can be achieved), (ii) protocol deemed feasible with minor modifications ( $\geq 75 \%$ of the criteria for success can be achieved), (iii) protocol deemed feasible with major modifications ( $\geq 50 \%$ of the criteria for success can be achieved), and (iv) protocol deemed not feasible (none of the criteria for success can be achieved) [32]. 


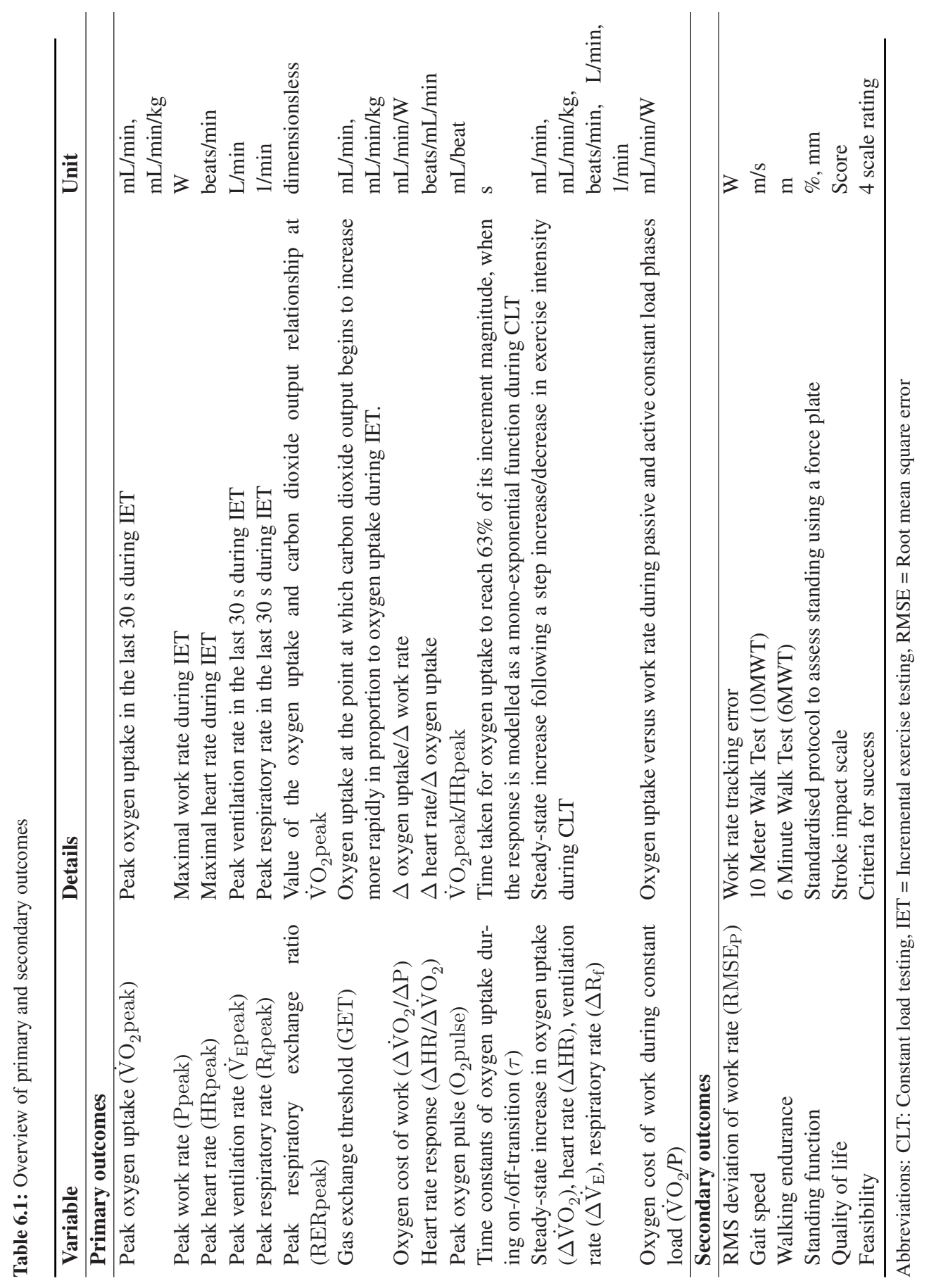




\section{Instrumentation}

We will use the Lokomat driven-gait orthosis system (Hocoma AG, Volketswil, Switzerland) integrated with a treadmill (h/p/cosmos sports medical GmbH, Traunstein, Germany) and a motordriven body weight support system with real time feedback control for precise body weight unloading (Lokolift, Hocoma AG) to apply RATE. A human-in-the-loop feedback system will control each subjects active work rate by projecting his mechanical work rate onto a screen at the front of the treadmill together with a target mechanical work rate. Figure 6.2 illustrates the FC-RATE approach. The subject will be instructed to vary the forces applied on the exoskeleton by volitional muscle activity and to keep the actual work rate as close as possible to the target. This procedure will be used to assess the primary outcome measures (aerobic capacity), and to guide each subjects individual target work rate during FC-RATE in the experimental group.

Pulmonary gas exchange and ventilatory measurements will be carried out using breath-bybreath spirometry (MetaMax 3B, Cortex Biophysik GmbH, Leipzig, Germany). Prior to each test, a volume calibration will be performed using a standardised volumetric syringe and gas calibration will be carried out using ambient air and a certified precision-analysed gas mixture. Heart rate will be continuously measured and recorded by a heart rate belt (T31, Polar Electro, Kempele, Finland) and a receiver board (HRMI, Sparkfun, Boulder, USA). Blood pressure will be monitored by a sphygmomanometer (HEM- 907, Omron Corporation, Kyoto, Japan). A software module for the overall procedure has been programmed in LabVIEW (Version 2009, National Instruments, Austin TX, USA) [33].

\section{Testing procedure}

The first RATE session will focus on adjusting the device to provide a physiological gait pattern, and on familiarisation with the facemask for respiratory monitoring. An initial test of decreasing body weight support (BWS) continuously by $5 \%$ each minute will be carried out to define individual BWS levels. Furthermore, we will implement a short period of incremental exercise to familiarise the subject with the feedback-control structure and to estimate dynamic maximal voluntary contraction during walking (MVC-W) within the exoskeleton to adjust the individual target work rate for the following exercise tests.

We will use constant load testing (CLT) [34] to implement a constant work load during RATE. Figure 6.3A shows a schematic representation of the CLT protocol that consists of: (1) a rest phase where subjects will stand on the treadmill attached to the exoskeleton for 5 minutes with $0 \% \mathrm{BWS}$; (2) a passive phase where subjects will walk passively with their individual BWS for 5 minutes; 


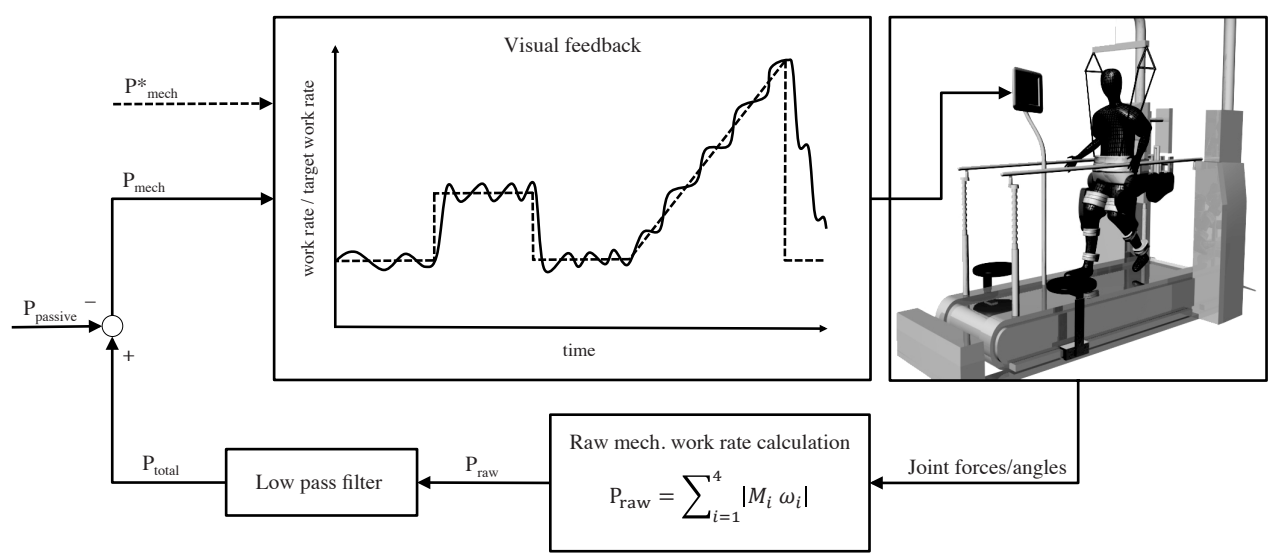

Figure 6.2: Human-in-the-loop work rate control during robotics-assisted treadmill exercise (RATE). The solid line represents the mechanical work rate $\left(\mathrm{P}_{\text {mech }}\right)$ produced by the subject, whereas the dashed line represents the target work rate $\left(\mathrm{P}_{\text {mech }}^{*}\right)$. The passive mechanical work rate $\left(\mathrm{P}_{\text {passive }}\right)$ is evaluated before every session and subtracted from $\mathrm{P}_{\text {mech }}$. Legend: $\mathrm{P}_{\text {raw }}=$ raw mechanical work rate, $M_{i}=$ moments of force, $\omega_{i}=$ angular velocity, $\mathrm{P}_{\text {total }}=$ total mechanical work rate.

and (3) an active phase where subjects will be instructed to actively contribute to the walking by pushing forward within the exoskeleton during the swing phase of each leg to reach the target work rate, which will be defined as $40 \%$ of MVC-W. The target work rate profile will be displayed on a screen in front of the subject. Using real-time visual feedback of their actual work rate the subjects will be asked to maintain their active contribution as close as possible to the target work rate. This phase will be terminated after 10 minutes; (4) passive phase/recovery: subjects will be instructed to walk passively with their individual BWS for 5 minutes; (5) rest: subjects will stand on the treadmill, still attached to the exoskeleton, for 5 minutes with $0 \%$ BWS.

Incremental exercise testing (IET) [35] will be used to estimate peak cardiopulmonary performance parameters. Figure 6.3B shows a schematic representation of the IET protocol that consists of: (1) a passive phase where subjects will be instructed to walk passively with their individual BWS for 5 minutes; (2) and active phase where subjects will be instructed to actively contribute to the walking by pushing forward within the exoskeleton during the swing phase of each leg to reach peak exercise capacity, where the slope will be defined so that MVC-W is reached in approximately 10 minutes; and (3) a passive phase/recovery where subjects will be instructed to walk passively with their individual BWS for 5 minutes. 
Both test protocols will follow strict termination criteria for cardiopulmonary exercise testing including: (1) abnormal blood pressure responses, that is, hypertensive (systolic BP $\geq 210 \mathrm{mmHg}$ or diastolic $\mathrm{BP} \geq 115 \mathrm{mmHg}$ ) when exercising at high work rate, or hypotensive responses (decrease in $\mathrm{BP} \geq 10 \mathrm{mmHg}$ ) despite an increase in work rate; (2) individual work rate below target work rate for $60 \mathrm{~s}$; (3) peak heart rate within 10 beats per minute of the age-predicted heart rate maximum [36], where the formula will be adjusted down to $70 \%$ of heart rate maximum for subjects on beta-blocker medications [37]; and (4) pain or discomfort in the chest. We will follow established models for cardiopulmonary exercise testing based on the ACSM guidelines [24].

The 10MWT and the 6MWT will be conducted using standardised testing protocols in a quiet area. Standing function will be assessed within parallel bars. The SIS will be administered to the subjects on a face-to-face basis.

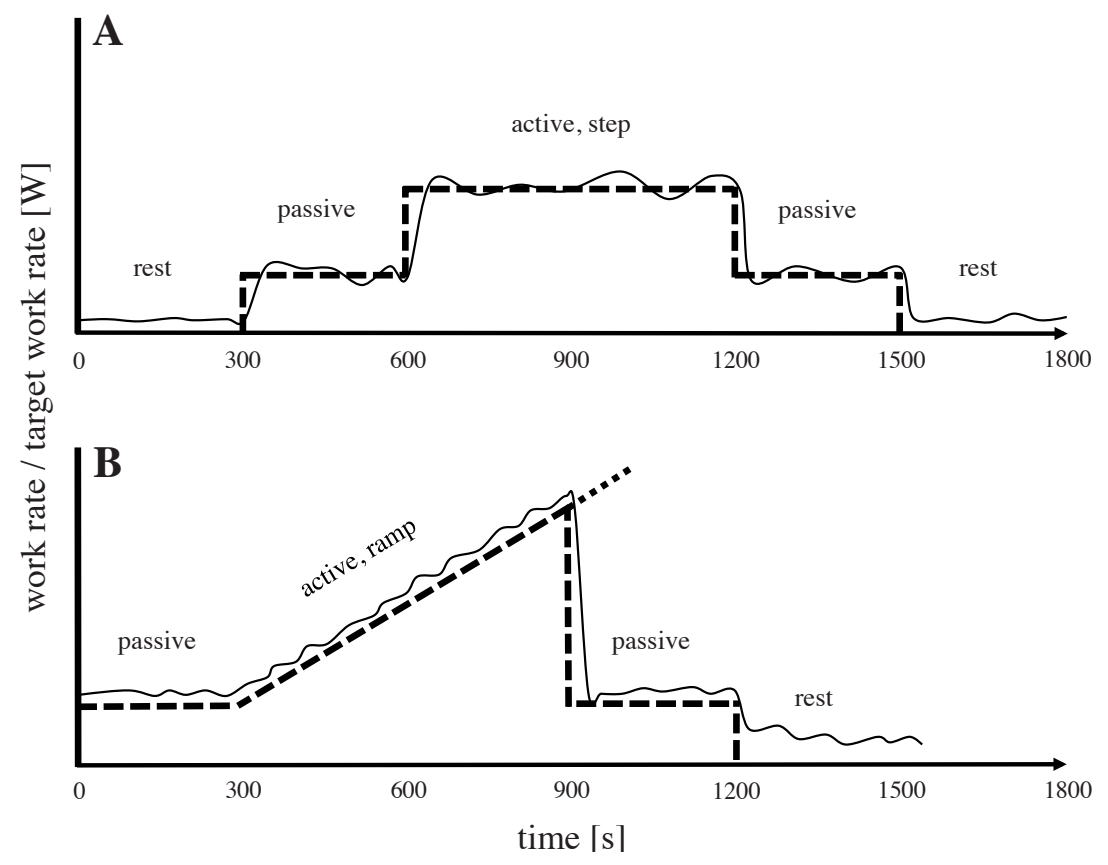

Figure 6.3: Exercise testing protocols. Schematic representation of constant load testing (A) and incremental exercise testing (B) using feedback-controlled robotics-assisted treadmill exercise. The solid line represents the mechanical work rate $\left(\mathrm{P}_{\text {mech }}\right)$ produced by the subject. The dashed line represents the target work rate $\left(\mathrm{P}_{\text {mech }}^{*}\right)$. The slope will be estimated such that the predefined peak work rate is reached after 10 minutes. When individual termination criteria are met the incremental phase is ended and $\mathrm{P}_{\text {mech }}^{*}$ set back to the passive level. 


\section{Intervention}

Both groups will receive regular RATE sessions (3/week, $30 \mathrm{~min} / \mathrm{session}$ ) during their inpatient rehabilitation period (typically, 4 to 12 weeks). Training intensity will be recorded by continuous heart rate monitoring and evaluating rate of perceived exertion (RPE) on the 6 to 20 scale [38]. The experimental group will receive progressive cardiovascular exercise using FC-RATE. Training will start at $40 \%$ of peak work rate (based on initial IET results) for the first session. Intensity will then be adjusted by increasing the target work rate in 5\% steps for subsequent sessions based on heart rate and RPE outcomes (target heart rate: 40 to $70 \%$ of heart rate reserve, target RPE: 11 to 14). The control group will receive conventional RATE, where therapists will focus on gait quality only. To confirm or refute low cardiovascular training intensity in the control group (target heart rate: $\leq 30 \%$ of heart rate reserve, target RPE: $\leq 7)$, therapists will monitor heart rate and RPE during all training sessions. All subjects will receive individually tailored conventional therapy including physiotherapy (4 to 5 sessions/week, 30 to $60 \mathrm{~min} / \mathrm{session}$ ), occupational therapy (2 to 3 sessions/week, 30 to $60 \mathrm{~min} / \mathrm{session}$ ), and individual speech and language therapy.

\section{Safety arrangements}

There is an inherent risk during cardiopulmonary exercise testing procedures and cardiovascular intervention programmes for subjects with subacute stroke of a recurrent cerebral or cardiac event. Furthermore, subjects are attached to the exoskeleton, which complicates emergency procedures. Several risk management arrangements will be implemented to ensure subjects safety: (1) clearly defined eligibility criteria to include medically stable subjects only, (2) continuous blood pressure monitoring during exercise testing (noted above), (3) presence of resuscitation-trained assistants, (4) opportunity to call the medical resuscitation team in the clinic, and (5) presence of personnel trained to release the subject within $60 \mathrm{~s}$ from the exoskeleton.

\section{Sample size and power calculation}

The required sample size has been calculated based on pooled estimation of standardised effect sizes obtained from a previous meta-analysis on cardiovascular exercise early after stroke [18]. Power analysis was done using $G^{*}$ Power Software (Version 3.1, University of Dusseldorf, Germany). A priori analysis indicated that a sample of at least 14 subjects would provide a statistical power of $80 \%$, that is, an $80 \%(\beta=0.2)$ chance to detect a $20 \%(\alpha=0.05)$ difference between groups. To allow for dropouts, a total of 20 subjects will be recruited. 


\section{Data management and analysis}

Data will be recorded on standardised forms and concealed between examiners and therapists. All data recorded continuously during testing will be saved automatically by the software module and will only be accessible by an independent software specialist. Data set examples will be checked for errors randomly or at any time a serious adverse event occurs. Raw breath-by-breath data will be processed using a zero phase shift moving average filter over 15 breaths [39]. Peak cardiopulmonary response variables will be calculated by analysing the final $30 \mathrm{~s}$ during incremental exercise, whereby criteria for maximal aerobic capacity will be (1) plateau in oxygen uptake, (2) respiratory exchange ratio (RER) $\geq 1.15$, and (3) peak heart rate within 10 beats per minute of the age-predicted heart rate maximum (adjusted for subjects on beta-blocker medications) [24]. Further peak performance variables will be defined as the maximal values at the termination time of the IET. The gas exchange threshold (GET) will be estimated using the v-slope method and standard gas exchange criteria [40]. Step response kinetics will be calculated using a non-linear least-squares algorithm to fit the data as described in the following mono-exponential equation: $\dot{\mathrm{VO}}_{2}(\mathrm{t})=\dot{\mathrm{VO}_{2}}(\mathrm{~b})+\dot{\mathrm{VO}}_{2}\left(1-\mathrm{e}^{-(\mathrm{t}-\mathrm{Td}) / \tau}\right), \mathrm{t}<0$ with $\dot{\mathrm{VO}}_{2}(\mathrm{~b})=\dot{\mathrm{V}} \mathrm{O}_{2}$ at baseline, $\Delta \dot{\mathrm{VO}}_{2}=$ step increase in oxygen uptake, $\mathrm{Td}=$ time delay of $20 \mathrm{~s}$ corresponding to the cardiodynamic phase of the response, and $\tau=$ time constant [34]. Steady-state will be defined as the mean value of the final $120 \mathrm{~s}$ during constant load phases. All analyses will be performed using MATLAB (Version R2010a, MathWorks, Natick MA, USA) and MetaSoft (Version 3.9.6 SR1, Cortex Biophysik GmbH, Leipzig, Germany).

\section{Statistical analysis}

Descriptive statistics will be calculated for dependent and independent variables. Demographic and clinical characteristics of the groups will be compared on admission using unpaired two-sample ttests (continuous data) and Chi-square analysis (categorical data). Nonparametric methods will be used when assumptions of normality are violated. To evaluate the clinical efficacy of the method, group differences will be compared after each 4-week intervention ( $\mathrm{t} 1, \mathrm{t} 2, \mathrm{t} 3$, etcetera) using unpaired two-sample t-tests for continuous data and z-tests for categorical data that conform to the assumption of normality. If data are not normally distributed for continuous variables, MannWhitney $U$ tests will be applied. A mixed effects analysis of variance with repeated measures to model the intervention effects over time for continuous variables that are normally distributed (that is, aerobic capacity) will be applied. Non-parametric tests (Mann-Whitney U and Kruskall Wallis tests) will be used for categorical data (that is, SIS) or where a normal distribution is not present. 
Variables that are significantly different in the baseline analysis will be used as covariates for further analysis. Multiple regressions will be applied to evaluate relationships among variables. All statistical analysis will be performed using SPSS (SPSS Inc., Chicago, Il, USA). The significance level will be 0.05 .

\section{Discussion}

The focus of this pilot trial is to evaluate the efficacy and the feasibility of cardiovascular exercise using FC-RATE with regard to aerobic capacity, motor recovery, and quality of life early after stroke. Study results will contribute to the evidence base regarding effects and feasibility of a novel cardiovascular exercise intervention for individuals suffering from severe motor impairment. Furthermore, the results will help the development of further methods for assessment of aerobic capacity and guidance of exercise intensity during early stroke rehabilitation.

It has previously been shown that cardiovascular exercise is effective in improving aerobic capacity and walking distance in individuals with moderate or low impairment after stroke [18]. However, there is a lack of appropriate intervention methods to include highly impaired individuals with low motor status. Chang et al. recently explored the effects of RATE on aerobic capacity and motor recovery early after stroke [41]. This conventional approach of reducing BWS and guidance torque, and increasing treadmill speed to guide exercise intensity during RATE showed improved aerobic capacity and increased lower extremity strength in non-ambulatory individuals. However, subjects received 2 weeks of regular exercise only, which is a relatively short aerobic training volume, and the guidance of exercise intensity using this conventional approach was reported to be very difficult.

The present pilot trial will implement a feedback-control structure for guidance of exercise intensity during RATE. This is the first controlled clinical application of this novel concept in an intervention study and might be a first step into intensity-guided cardiovascular rehabilitation research for individuals suffering from severe motor impairment early after stroke. The long-term objective of this research programme is to provide a basis for exploration of the association of early cardiovascular activation/exercise and neural plasticity. There are a few studies that support this objective by reporting promising effects of early cardiovascular exercise on cognition [42], motor function of the lower extremities [43, 44], and functional independence [45]. The present trial could provide new insights into cardiovascular rehabilitation early after stroke and may, therefore, guide future research on this topic. This will be relevant for further development of rehabilitation robotics and future stroke rehabilitation strategies. 


\section{Conclusion}

Our overall research plan aims to further develop and implement robotics-assisted devices for cardiovascular rehabilitation soon after stroke. The approach presented here has been tested previously in a phase II clinical trial that has shown feasibility of the concept for assessment of aerobic capacity and guidance of cardiovascular exercise in severely impaired individuals. The phase III clinical trial described here aims to determine early indications of the presence and magnitude of efficacy as well as to refine the definition of the experimental protocol. If this trial is able to show reasonable clinical feasibility and to demonstrate having potential for efficacy on the primary outcomes (aerobic capacity), further full-scale trials would be warranted to investigate overall effectiveness of this approach. In case of poor feasibility and low efficacy, the concept might have to undergo further revision and development to optimally support cardiovascular exercise in severely impaired populations.

\section{Trial status}

The trial was designed and commenced in 2012. The expected time of completion of enrolment is June 2014.

\section{References}

[1] "World health statistics," World Health Organization, 2012.

[2] G. A. Donnan, M. Fisher, M. Macleod, and S. M. Davis, "Stroke," Lancet, vol. 371, no. 9624, pp. 1612-1623, 2008.

[3] M. Koivusalo and M. Mackintosh, "The world health report 2007: A safer future: Global public health security in the 21st century," Development and Change, vol. 39, no. 6, pp. 1163-1169, 2008.

[4] W. Rosamond, K. Flegal, G. Friday, K. Furie, A. Go, K. Greenlund, N. Haase, M. Ho, V. Howard, B. Kissela, S. Kittner, D. Lloyd-Jones, M. McDermott, J. Meigs, C. Moy, G. Nichol, C. J. O’Donnell, V. Roger, J. Rumsfeld, P. Sorlie, J. Steinberger, T. Thom, S. Wasserthiel-Smoller, Y. L. Hong, and A. Amer Heart, "Heart disease and stroke statistics - 2007 update - a report from the american heart association statistics committee and stroke statistics subcommittee," Circulation, vol. 115, no. 5, pp. E69-E171, 2007.

[5] A. Di Carlo, "Human and economic burden of stroke," Age and Ageing, vol. 38, no. 1, pp. 4-5, 2009.

[6] E. J. Roth, "Heart disease in patients with stroke: incidence, impact, and implications for rehabilitation. part 1: Classification and prevalence," Archives of Physical Medicine and Rehabilitation, vol. 74, no. 7, pp. 752-760, 1993.

[7] E. J. Roth, "Heart disease in patients with stroke. part 2: Impact and implications for rehabilitation," Archives of Physical Medicine and Rehabilitation, vol. 75, no. 1, pp. 94-101, 1994. 
[8] C. E. Hafer-Macko, A. S. Ryan, F. M. Ivey, and R. F. Macko, "Skeletal muscle changes after hemiparetic stroke and potential beneficial effects of exercise intervention strategies," Journal of Rehabilitation Research and Development, vol. 45, no. 2, pp. 261-272, 2008.

[9] J. O. Kelly, S. L. Kilbreath, G. M. Davis, B. Zeman, and J. Raymond, "Cardiorespiratory fitness and walking ability in subacute stroke patients," Archives of Physical Medicine Rehabilitation, vol. 84, no. 12, pp. 1780$1785,2003$.

[10] M. J. MacKay-Lyons and L. Makrides, "Cardiovascular stress during a contemporary stroke rehabilitation program: Is the intensity adequate to induce a training effect?," Archives of Physical Medicine and Rehabilitation, vol. 83, no. 10, pp. 1378-1383, 2002.

[11] A. Tang, K. M. Sibley, S. G. Thomas, W. E. McIlroy, and D. Brooks, "Maximal exercise test results in subacute stroke," Archives of Physical Medicine and Rehabilitation, vol. 87, no. 8, pp. 1100-1105, 2006.

[12] D. Brooks, A. Tang, K. M. Sibley, and W. E. McIlroy, "Profile of patients at admission into an inpatient stroke rehabilitation programme: cardiorespiratory fitness and functional characteristics," Physiotherapy Canada, vol. 60, no. 2, pp. 171-179, 2008.

[13] J. J. Eng, A. S. Dawson, and K. S. Chu, "Submaximal exercise in persons with stroke: Test-retest reliability and concurrent validity with maximal oxygen consumption," Archives of Physical Medicine and Rehabilitation, vol. 85, no. 1, pp. 113-118, 2004.

[14] K. Potempa, M. Lopez, L. T. Braun, J. P. Szidon, L. Fogg, and T. Tincknell, "Physiological outcomes of aerobic exercise training in hemiparetic stroke patients," Stroke, vol. 26, no. 1, pp. 101-105, 1995.

[15] F. M. Ivey, R. F. Macko, A. S. Ryan, and C. E. Hafer-Macko, "Cardiovascular health and fitness after stroke," Topics in Stroke Rehabilitation, vol. 12, no. 1, pp. 1-16, 2005.

[16] J. Ramas, A. Courbon, F. Roche, F. Bethoux, and P. Calmels, "Effect of training programs and exercise in adult stroke patients: literature review," Annales de Readaptation et de Medecine Physique, vol. 50, no. 6, pp. 438444, 2007.

[17] E. J. Roth, K. Mueller, and D. Green, “Stroke rehabilitation outcome - impact of coronary-artery disease,” Stroke, vol. 19 , no. 1, pp. 42-47, 1988.

[18] O. Stoller, E. D. de Bruin, R. H. Knols, and K. J. Hunt, "Effects of cardiovascular exercise early after stroke: systematic review and meta-analysis," BMC Neurology, vol. 12, no. 1, p. 45, 2012.

[19] D. T. Wade, V. A. Wood, A. Heller, J. Maggs, and R. L. Hewer, "Walking after stroke - measurement and recovery over the 1st 3 months," Scandinavian Journal of Rehabilitation Medicine, vol. 19, no. 1, pp. 25-30, 1987.

[20] H. S. Jorgensen, H. Nakayama, H. O. Raaschou, J. Vivelarsen, M. Stoier, and T. S. Olsen, "Outcome and time course of recovery in stroke. part ii: Time course of recovery. the copenhagen stroke study," Archives of Physical Medicine and Rehabilitation, vol. 76, no. 5, pp. 406-412, 1995.

[21] K. J. Hunt, L. P. Jack, A. Pennycott, C. Perret, M. Baumberger, and T. H. Kakebeeke, "Control of work ratedriven exercise facilitates cardiopulmonary training and assessment during robot-assisted gait in incomplete spinal cord injury," Biomedical Signal Processing and Control, vol. 3, no. 1, pp. 19-28, 2008.

[22] L. P. Jack, M. Purcell, D. B. Allan, and K. J. Hunt, "Comparison of peak cardiopulmonary performance parameters during robotics-assisted treadmill exercise and arm crank ergometry in incomplete spinal cord injury," Technology and Health Care, vol. 18, no. 4-5, pp. 285-296, 2010.

[23] O. Stoller, M. Schindelholz, L. Bichsel, C. Schuster, R. A. de Bie, E. D. de Bruin, and K. J. Hunt, "Feedbackcontrolled robotics-assisted treadmill exercise to assess and influence aerobic capacity early after stroke: a proofof-concept study," Disabil Rehabil Assist Technol, vol. 9, no. 4, pp. 271-278, 2014. 
[24] W. R. Thompson, N. F. Gordon, and L. S. Pescatello, American College of Sports Medicine. Guidelines for Exercise Testing and Prescription. Philadelphia: Lippincott Williams Wilkins, eight ed., 2010.

[25] "Ats/accp statement on cardiopulmonary exercise testing," American Journal of Respiratory and Critical Care Medicine, vol. 167, pp. 211-277, 2003.

[26] F. M. Collen, D. T. Wade, and C. M. Bradshaw, "Mobility after stroke: reliability of measures of impairment and disability," Int Disabil Stud, vol. 12, no. 1, pp. 6-9, 1990.

[27] J. H. Lin, M. J. Hsu, H. W. Hsu, H. C. Wu, and C. L. Hsieh, "Psychometric comparisons of 3 functional ambulation measures for patients with stroke," Stroke, vol. 41, no. 9, pp. 2021-2025, 2010.

[28] G. D. Fulk and J. L. Echternach, “Test-retest reliability and minimal detectable change of gait speed in individuals undergoing rehabilitation after stroke," J Neurol Phys Ther, vol. 32, no. 1, pp. 8-13, 2008.

[29] M. Kosak and T. Smith, "Comparison of the 2-, 6-, and 12-minute walk tests in patients with stroke," J Rehabil Res Dev, vol. 42, no. 1, pp. 103-107, 2005.

[30] A. Ruhe, R. Fejer, and B. Walker, "The test-retest reliability of centre of pressure measures in bipedal static task conditions - a systematic review of the literature," Gait Posture, vol. 32, no. 4, pp. 436-445, 2010.

[31] P. W. Duncan, D. Wallace, S. M. Lai, D. Johnson, S. Embretson, and L. J. Laster, "The stroke impact scale version 2.0 - evaluation of reliability, validity, and sensitivity to change," Stroke, vol. 30, no. 10, pp. 2131-2140, 1999.

[32] L. Thabane, J. Ma, R. Chu, J. Cheng, A. Ismaila, L. P. Rios, R. Robson, M. Thabane, L. Giangregorio, and C. H. Goldsmith, "A tutorial on pilot studies: the what, why and how," BMC Med Res Methodol, vol. 10, p. 1, 2010.

[33] M. Schindelholz, O. Stoller, and K. J. Hunt, "A software module for cardiovascular rehabilitation in roboticsassisted treadmill exercise,” Biomedical Signal Processing and Control, vol. 10, pp. 296-307, 2014.

[34] B. J. Whipp and K. Wasserman, “Oxygen-uptake kinetics for various intensities of constant-load work,” Journal of Applied Physiology, vol. 33, no. 3, pp. 351-356, 1972.

[35] B. J. Whipp, J. A. Davis, F. Torres, and K. Wasserman, "A test to determine parameters of aerobic function during exercise," Journal of Applied Physiology, vol. 50, no. 1, pp. 217-217, 1981.

[36] H. Tanaka, K. D. Monahan, and D. R. Seals, "Age-predicted maximal heart rate revisited," Journal of the American College of Cardiology, vol. 37, no. 1, pp. 153-156, 2001.

[37] P. A. Tesch, “Exercise performance and beta-blockade," Sports Medicine, vol. 2, no. 6, pp. 389-412, 1985.

[38] G. A. Borg, “Perceived exertion,” Exerc Sport Sci Rev, vol. 2, pp. 131-153, 1974.

[39] R. A. Robergs, D. Dwyer, and T. Astorino, "Recommendations for improved data processing from expired gas analysis indirect calorimetry," Sports Medicine, vol. 40, no. 2, pp. 95-111, 2010.

[40] W. L. Beaver, K. Wasserman, and B. J. Whipp, "A new method for detecting anaerobic threshold by gasexchange,” Journal of Applied Physiology, vol. 60, no. 6, pp. 2020-2027, 1986.

[41] W. H. Chang, M. S. Kim, J. P. Huh, P. K. W. Lee, and Y. H. Kim, "Effects of robot-assisted gait training on cardiopulmonary fitness in subacute stroke patients: A randomized controlled study," Neurorehabilitation and Neural Repair, vol. 26, no. 4, pp. 318-324, 2012.

[42] M. Ploughman, J. McCarthy, M. Bosse, H. J. Sullivan, and D. Corbett, "Does treadmill exercise improve performance of cognitive or upper-extremity tasks in people with chronic stroke? a randomized cross-over trial," Archives of Physical Medicine and Rehabilitation, vol. 89, no. 11, pp. 2041-2047, 2008. 
[43] P. Duncan, L. Richards, D. Wallace, J. Stoker-Yates, P. P. Pohl, C. Luchies, A. Ogle, and S. Studenski, “A randomized, controlled pilot study of a home-based exercise program for individuals with mild and moderate stroke," Stroke, vol. 29, no. 10, pp. 2055-2060, 1998.

[44] P. Duncan, S. Studenski, L. Richards, S. Gollub, S. M. Lai, D. Reker, S. Perera, J. Yates, V. Koch, S. Rigler, and D. Johnson, "Randomized clinical trial of therapeutic exercise in subacute stroke," Stroke, vol. 34, no. 9, pp. 2173-2180, 2003.

[45] A. Letombe, C. Cornille, H. Delahaye, A. Khaled, O. Morice, A. Tomaszewski, and N. Olivier, "Early poststroke physical conditioning in hemiplegic patients: A preliminary study," Annals of Physical Rehabilitation Medicine, vol. 53, no. 10, pp. 632-642, 2010. 


\title{
Chapter 7
}

\section{Efficacy of feedback-controlled robotics-assisted treadmill exercise to improve cardiovascular fitness early after stroke: a randomised controlled pilot trial}

Journal of Neurologic Physical Therapy Submitted - in revision

\author{
Oliver Stoller \\ Eling D de Bruin \\ Matthias Schindelholz \\ Corina Schuster-Amft \\ Rob A de Bie \\ Kenneth J Hunt
}




\section{Abstract}

Background and Purpose: Cardiovascular fitness is seriously reduced after stroke. While individuals with mild to moderate impairments benefit from conventional cardiovascular exercise interventions, there is a lack of effective concepts for the severely impaired population. This randomised controlled pilot trial investigated efficacy and feasibility of feedback-controlled roboticsassisted treadmill exercise (FC-RATE) for cardiovascular rehabilitation in persons with severe impairments early after stroke.

Methods: Twenty individuals (age $61 \pm 11$ years, $52 \pm 31$ days post-stroke) with severe motor limitations (Functional Ambulation Classification 0-2) were recruited for FC-RATE or conventional robotics-assisted treadmill exercise (RATE) (4 weeks, 3x30 min sessions/week). Outcome measures focused on peak cardiopulmonary performance parameters, training intensity, and feasibility, with examiners blinded to allocation.

Results: All 14 allocated participants (70\%) completed the intervention (7/group, withdrawals unrelated to the intervention), without serious adverse events occurring. Cardiovascular fitness increased significantly in both groups, with peak oxygen uptake increasing from 14.6 to 17.7 $\mathrm{mL} / \mathrm{min} / \mathrm{kg}(+17.8 \%)$ after 4 weeks ( 45.8 to $55.7 \%$ of predicted maximal aerobic capacity) (time effect $\mathrm{p}=0.01$, no group-time interaction). Training intensity (\% heart rate reserve) was significantly higher for FC-RATE ( $40 \pm 3 \%)$ compared to conventional RATE $(14 \pm 2 \%)(\mathrm{p}=0.001)$.

Discussion and Conclusions: Substantial and significant overall increases in the main cardiopulmonary performance parameters were observed, but there were no significant between-group differences when comparing FC-RATE and conventional RATE. FC-RATE significantly increased exercise intensity, but recommended intensity levels for cardiovascular training were not consistently achieved. Future research should focus on appropriate algorithms within advanced robotic systems to promote optimal cardiovascular stress.

Trial registration: ClinicalTrials.gov Registry (NCT01679600) 


\section{Introduction}

Evidence suggests that individuals with stroke benefit from cardiovascular exercise interventions. Several prospective trials and meta-analyses investigating cardiovascular fitness interventions using leg cycle ergometry, treadmill training, and combined upper- and lower-limb ergometry demonstrated beneficial effects on cardiovascular fitness in subacute $[1,2,3,4,5,6,7,8,9,10,11,12]$ and chronic stroke $[13,14,15,16,17]$. Furthermore, preliminary evidence exists for improved cerebral vasomotor reactivity [18], subcortical neural network activation [19], and enhanced cognitive function [20, 21] following cardiovascular exercise post-stroke. However, the main body of research focused on individuals with mild to moderate motor impairments. Unique challenges such as non-ambulatory status, limited trunk control, poor postural control, and poor coordination of the affected limbs may restrict individuals with severe impairments from performing exercise on conventional devices. Recently, a total-body recumbent stepper to implement cardiovascular exercise in persons with severe motor limitations was proposed [22, 23]. Considering that walking ability is one of the major goals of stroke rehabilitation, and given the importance of task-specific training in the early stages of recovery [24, 25], more functional rehabilitation strategies are sought to provide effective exercise interventions in populations with severe motor impairment.

A promising approach to overcome motor limitations while facilitating task-specific activity and cardiovascular stress is body weight supported treadmill training. Research has shown that gait symmetry improved with increasing body weight support [26]. However, during walking with body weight support of more than $15 \%$, vertical ground reaction forces and functional activity of antigravity muscles are decreased, which led to substantially lower oxygen uptake levels during body weight supported treadmill training compared to conventional treadmill exercise [27, 28]. Because individuals with severe motor impairments need considerable guidance during walking with lower body weight support, the application of robotics-assisted treadmill exercise (RATE) might be of relevance in this context. The technology provides important features for neuromuscular and cardiovascular training, i.e. assistive support during the gait trajectory for progressive body weight loading, and the opportunity to implement control strategies to facilitate participation and increase effort.

Studies on motor recovery after stroke have shown that RATE appears to facilitate similar outcomes compared to body weight supported treadmill training [29, 30, 31]. However, individuals tend to minimise their effort during RATE due to the constant physical support of the actuated exoskeleton, defined as "slacking" [32]. Metabolic cost and muscle activity patterns have been shown to be substantially lower during RATE compared to conventional body weight supported treadmill 
training in persons with incomplete spinal cord injury [33]. Nevertheless, recent experimental research on cardiovascular responses in persons with severe hemiplegia significantly increased the metabolic cost during RATE $[34,35,36]$. The active stance phase which produces body weight loading might be responsible for the increase in exercise intensity, while gait speed and advanced force controllers (e.g. guidance force) had only small effects on oxygen uptake [34].

Optimal exercise intensity is a crucial factor for improving cardiovascular fitness. Recent guidelines for individuals with stroke recommend regular cardiovascular exercise at an intensity level of $40-70 \%$ heart rate reserve (55-80\% of heart rate maximum; rate of perceived exertion 11-14 (6-20 scale)) [37]. Although a first intervention study on RATE to improve cardiovascular fitness by decreasing body weight support yielded promising results in the subacute phase after stroke [38], further research has shown that conventional RATE could not reach recommended cardiovascular exercise intensity in persons with severe hemiplegia [35, 36]. Therefore, a novel protocol has been developed to control and direct active participation during RATE with the specific aim of provoking higher cardiopulmonary responses [39]. This incorporates biofeedback mechanisms allowing the control of exercise intensity through the guidance of the individual's voluntary effort. The approach presented here provides control of exercise intensity during RATE by biofeedback and voluntary adaptation of the hip and knee forces by the individual. Initial studies demonstrated feasibility and reliability for feedback-controlled RATE (FC-RATE) to assess cardiovascular fitness and guide exercise intensity during CPET protocols soon after stroke $[40,41]$. The next logical step is to evaluate FC-RATE in a pilot randomised controlled trial in order to assess the clinical efficacy and feasibility of the method for cardiovascular training and improving cardiovascular fitness during subacute stroke rehabilitation [42]. This is relevant for the further development of the concept and the design of future large-scale trials.

The aim of this study was to carry out a preliminary investigation of efficacy and feasibility of FC-RATE for improving cardiovascular fitness in persons with severe motor impairments early after stroke. We hypothesised that FC-RATE would reach a substantially higher cardiovascular training intensity compared to conventional RATE in a clinical setting, thus resulting in significantly increased cardiovascular fitness following a 4-week FC-RATE intervention. In addition, we expected to affirm feasibility by achieving predefined criteria. 


\section{Methods}

\section{Design}

This was a single-centre, single blind, randomised control pilot trial. The study protocol was published previously [43]. The local ethics committee approved all experimental procedures (Reference No. 2012/051).

\section{Participants}

Twenty inpatients with first-ever stroke were recruited by research staff at a neurological rehabilitation clinic in Switzerland between October 2012 and March 2014. Participants were presented to the responsible ward physician and a cardiologist to confirm eligibility. Inclusion criteria were: (1) clinical diagnosis of a first-ever stroke, (2) $<20$ weeks post-stroke, (3) age $>18$ years, (4) Functional Ambulation Classification $<3,(5)$ ability to understand the procedures and provide informed consent. Exclusion criteria were: (1) contraindications for CPET as outlined by the American College of Sports Medicine [44], (2) contraindications for RATE according to the device manufacturer (e.g. bone instability (osteoporosis), severely fixed contractures, vascular disorders of the lower limbs, etc.) , (3) concurrent neurological disease (e.g. spinal cord injury, multiple sclerosis, Parkinsons disease, etc.), (4) concurrent pulmonary disease (e.g. COPD, etc.), (5) history of dementia. Individuals with any suspicion of these conditions were excluded. All participants provided written informed consent.

Randomisation was performed using a computer-generated permuted 4-block allocation scheme. A study-independent researcher generated the list and sent it to the clinic's pharmacy for safekeeping. The leading trial therapist contacted the pharmacy for assignment. A blinded examiner conducted all assessments and the randomisation was concealed until the last post-intervention assessment was performed and the data processing and acquisition was terminated.

\section{Outcomes}

The primary outcome of this study was efficacy, which was evaluated by cardiovascular fitness and training intensity. Peak cardiopulmonary performance was assessed by peak oxygen uptake $\left(\dot{\mathrm{V}}_{2}\right.$ peak), peak work rate (Ppeak), peak ventilation rate $\left(\dot{\mathrm{V}}_{\mathrm{E}}\right.$ peak $)$, peak respiratory rate $\left(\mathrm{R}_{\mathrm{f}}\right.$ peak), peak heart rate (HRpeak), and respiratory exchange ratio $\left(\dot{\mathrm{V}} \mathrm{CO}_{2} / \mathrm{V}_{2}\right)$ at $\dot{\mathrm{V}} \mathrm{O}_{2}$ peak (RERpeak). Additional cardiovascular parameters included the gas exchange threshold (GET) 
and important derivatives such as $\mathrm{O}_{2}$ cost of work $\left(\Delta \dot{\mathrm{V}} \mathrm{O}_{2} / \Delta \mathrm{P}\right), \mathrm{O}_{2}$ pulse at $\dot{\mathrm{V}} \mathrm{O}_{2}$ peak $\left(\mathrm{O}_{2}\right.$ pulse $)$ and $\dot{\mathrm{V}}_{\mathrm{E}}$ versus $\dot{\mathrm{VCO}}_{2}$ slope $\left(\Delta \dot{\mathrm{V}}_{\mathrm{E}} / \Delta \dot{\mathrm{V}} \mathrm{CO}_{2}\right)$. Continuous heart rate monitoring was used to evaluate the training intensity. The secondary outcome was feasibility, which was rated based on predefined criteria [45]. Compliance focused on training attendance during the intervention phase, i.e. the number of sessions completed (defined to be $\geq 90 \%$ ). The number of dropouts gave the attrition rate during the entire study (defined to be $\leq 10 \%$ ). The occurrence of any serious adverse events gave information on the protocol's clinical safety (defined to be 100\%). Successful data acquisition was assessed by reporting any loss of breath-by-breath, continuous heart rate, or mechanical work rate data (defined to be $100 \%$ ).

\section{Instrumentation}

RATE was implemented using the Lokomat system (Hocoma AG, Volketswil, Switzerland). The powered exoskeleton provides control of both legs, synchronised with an integrated treadmill (h/p/cosmos sports medical GmbH, Traunstein, Germany) and a motor-driven body weight support system with real time feedback control for precise body weight unloading (Lokolift, Hocoma AG). The feedback-control approach for exercise testing and experimental training was based on the work rate exerted on the exoskeleton by the participant calculated from the force, moment arm and velocity data at the 4 active joints (hip and knee). The participant was instructed to vary the forces applied on the exoskeleton by volitional muscle activity and to keep the measured and visualised active work rate as close as possible to the target (Figure 7.1). Detailed information on FC-RATE can be found elsewhere [40].

Pulmonary gas exchange and ventilatory measurements were carried out using breath-bybreath ergospirometry (MetaMax 3B, Cortex Biophysik GmbH, Leipzig, Germany). Heart rate was recorded by a heart rate belt (T31, Polar Electro, Kempele, Finland) and a receiver board (HRMI, Sparkfun, Boulder, USA). A software module for the overall procedure was programmed in LabVIEW (Version 2009, National Instruments, Austin TX, USA) [46].

\section{Testing procedure}

Cardiovascular fitness was assessed using FC-RATE-based CPET. At study entry, all participants completed a familiarisation session with the FC-RATE concept, starting by qualified and experienced physiotherapists adjusting the Lokomat system to provide a physiological gait pattern and ensuring the participants could walk comfortably. An initial test of decreasing body weight support continuously by $5 \%$ per minute was implemented to define the minimal possible body weight 


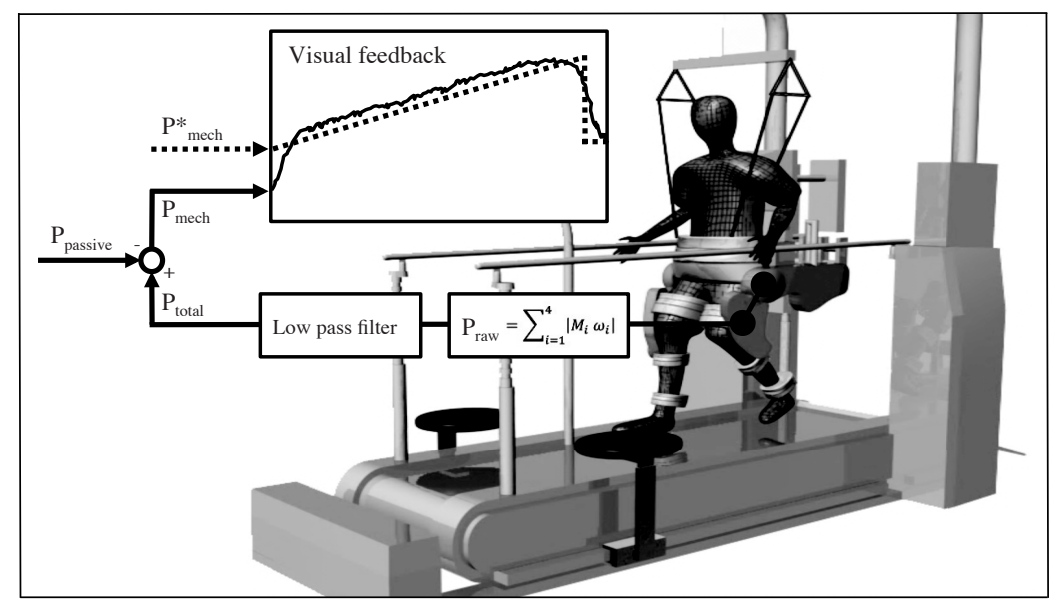

Figure 7.1: Feedback-controlled robotics-assisted treadmill exercise. Hip and knee joint forces and angles are measured in real time to allow calculation of the mechanical work rate $\left(\mathrm{P}_{\text {mech }}\right.$, solid line $)$ and projection onto a screen in front of the person. Individual target work rate profiles $\left(\mathrm{P}_{\text {mech }}^{*}\right.$, dashed line $)$ are used to guide exercise intensity during robotics-assisted walking. The passive mechanical work rate $\left(\mathrm{P}_{\text {passive }}\right)$ is evaluated before every session and subtracted from $\mathrm{P}_{\text {mech }}$. Legend: $\mathrm{P}_{\text {raw }}=$ raw mechanical work rate, $M_{i}=$ moments of force, $\omega_{i}=$ angular velocity, $\mathrm{P}_{\text {total }}=$ total mechanical work rate.

support level. After the first adjustments, participants were asked to perform a short constant load exercise test for $5 \mathrm{~min}$ (target work rate $=20 \mathrm{~W}$ ) in order to practice using the feedback-control structure. Finally, the safety procedures for potential adverse events were explained in detail.

After a break of at least $24 \mathrm{~h}$, participants performed consecutive CPET with 48-72 $\mathrm{h}$ between the trials. All sessions were controlled for time of day. Participants were instructed not to consume food, alcohol, nicotine or caffeine at least $3 \mathrm{~h}$ prior to testing.

Participants were asked at the beginning of the first exercise test to increase their maximal voluntary effort during FC-RATE within $30 \mathrm{~s}$ to define the maximal work rate for the subsequent tests. The progressive ramp was defined as a continuous slope aiming to reach the predefined maximal work rate in $10 \mathrm{~min}$. All participants performed 2 tests at baseline (on separate days) and 1 test at post-intervention. The test protocol followed strict termination criteria and there was close adherence to established models for CPET according to international guidelines [44, 47]. Detailed information on FC-RATE-based CPET can be found elsewhere [41]. 


\section{Intervention}

Both groups were balanced in terms of exposure (3/week, $30 \mathrm{~min} / \mathrm{session}$ ) over a period of 4 weeks (12 sessions). All training sessions were led by 1 of 2 experienced physiotherapists, who were trained in the intervention protocols. The training intensity was monitored in both groups by continuous heart rate measurement and further confirmed by rating of perceived exertion on the 6-20 Borg scale [48]. Walking cadence was fixed at $60 \mathrm{steps} / \mathrm{min}$ for all sessions and body weight support was individually adjusted within a range of $\pm 10 \%$ to provide a physiological gait pattern.

The experimental group received progressive cardiovascular exercise using FC-RATE. The first training session was defined to start at $40 \%$ of Ppeak to approach $40 \%$ of heart rate reserve (Borg scale approximately 11-14). The intensity was then adjusted based on continuous heart rate data and rating of perceived exertion indications by modulating the target work rate in 5\% increments for every subsequent session to reach the target intensity. The target heart rate was set at $40-70 \%$ of heart rate reserve [37]. The control group received conventional RATE, where therapists focused on gait quality only. All training sessions started with a 5 min warm-up period while passively walking in the device, followed by 25 min of continuous FC-RATE at the target level (experimental) or 25 min of conventional RATE (control).

All participants received individually tailored conventional care, in addition to RATE or FCRATE, including physiotherapy (4-5 sessions/week, 30-60 min/session), occupational therapy (23 sessions/week, 30-60 min/session), and individual speech and language therapy. Due to the severely impaired status of the participants, no specific aerobic exercise training was provided during conventional care.

\section{Data processing}

Raw breath-by-breath data were processed using a zero phase shift moving average filter over 15 breaths [49]. Peak cardiopulmonary variables were defined as the maximal values in the final 30 $\mathrm{s}$ during incremental exercise. Of the 2 consecutive tests at baseline, the data set containing the highest $\dot{\mathrm{VO}_{2}}$ peak was considered for analysis. Criteria for maximal aerobic capacity were: (1) plateau in oxygen uptake, (2) RER $\geq 1.15$, and (3) HRpeak within 10 beats per minute of the agepredicted heart rate maximum (adjusted for participants on beta-blocker medications) [44]. The identification of a $\dot{\mathrm{VO}} 2$ plateau was performed by plotting the slope and $95 \%$ confidence interval (CI) of the $\Delta \dot{\mathrm{VO}}_{2} / \Delta \mathrm{P}$ slope, where data points outside the extrapolated $95 \% \mathrm{CI}$ were taken as evidence of plateauing or leveling off behavior [50]. The GET was estimated using the v-slope method [51]. Predicted maximal heart rate was defined as $208-(0.7 \mathrm{x}$ age $)$ [52], but the formula 
was adjusted to $70 \%$ for participants on beta-blocker medication [53]. Heart rate reserve was defined as predicted maximal heart rate - resting heart rate [54]. Data processing was performed using MATLAB (Version R2010a, MathWorks, Natick MA, USA) and LabVIEW (Version 2009, National Instruments, Austin TX, USA).

\section{Statistical analysis}

Descriptive statistics were calculated on all outcome variables. For comparisons of baseline characteristics and training intensity between-groups, a Mann-Whitney U-test was applied for continuous and ordinal variables and $\mathrm{a}^{2}$ test for nominal variables. The analyses were based on the intention-to-treat approach. The F1-LD-F1 model by Brunner and Langer was used to test the median treatment effect, time effect, and the effect of their interaction by an analysis of variance (ANOVA)-type test statistic (Fn) [55]. The models were computed with the R 3.0.3. statistics program. All other statistical analyses were performed with SPSS (SPSS Inc., Chicago, United States). Due to the exploratory nature of the study, two-sided $\mathrm{p}$-values $\mathrm{p} \leq 0.05$ were considered significant and no adjustment for multiple endpoints was performed.

\section{Results}

\section{Participants}

The number of individuals screened, enrolled, randomised, and completing the trial is shown in Figure 7.2. There were no significant differences in baseline characteristics between the two groups (Table 7.1). No serious adverse events occurred during FC-RATE-based CPET. Thirteen participants (93\%) completed the tests without symptomatic responses. The overall reason for test termination was inability to reach the target work rate due to generalised and/or leg fatigue.

One participant required a test termination per safety criteria due to high systolic blood pressure of $>210 \mathrm{mmHg}$ during the baseline assessments, which was considered an adverse event. The participant's results were considered valid, because both exercise tests were terminated at very high exercise intensity. The responsible ward physician/surgeon admitted the participant to the intervention phase after careful medical evaluation. The high systolic blood pressure response in this participant did not occur during the post-intervention assessment.

With respect to the 3 criteria for maximal aerobic capacity, 2 participants (14\%) showed a plateau in $\dot{\mathrm{VO}}_{2}, 1$ participant (7\%) achieved an RERpeak value $\geq 1.15$, and 4 participants (29\%) reached HRpeak within 10 beats per minute of the age-predicted heart rate maximum; 2 of these 
had an adjusted heart rate due to beta-blocker medication. None of the participants reached more than 1 criterion for maximal aerobic capacity. No significant differences were found across cardiopulmonary performance parameters at baseline, except for $\Delta \dot{\mathrm{V}} \mathrm{O}_{2} / \Delta \mathrm{P}(\mathrm{p}=0.017)$.

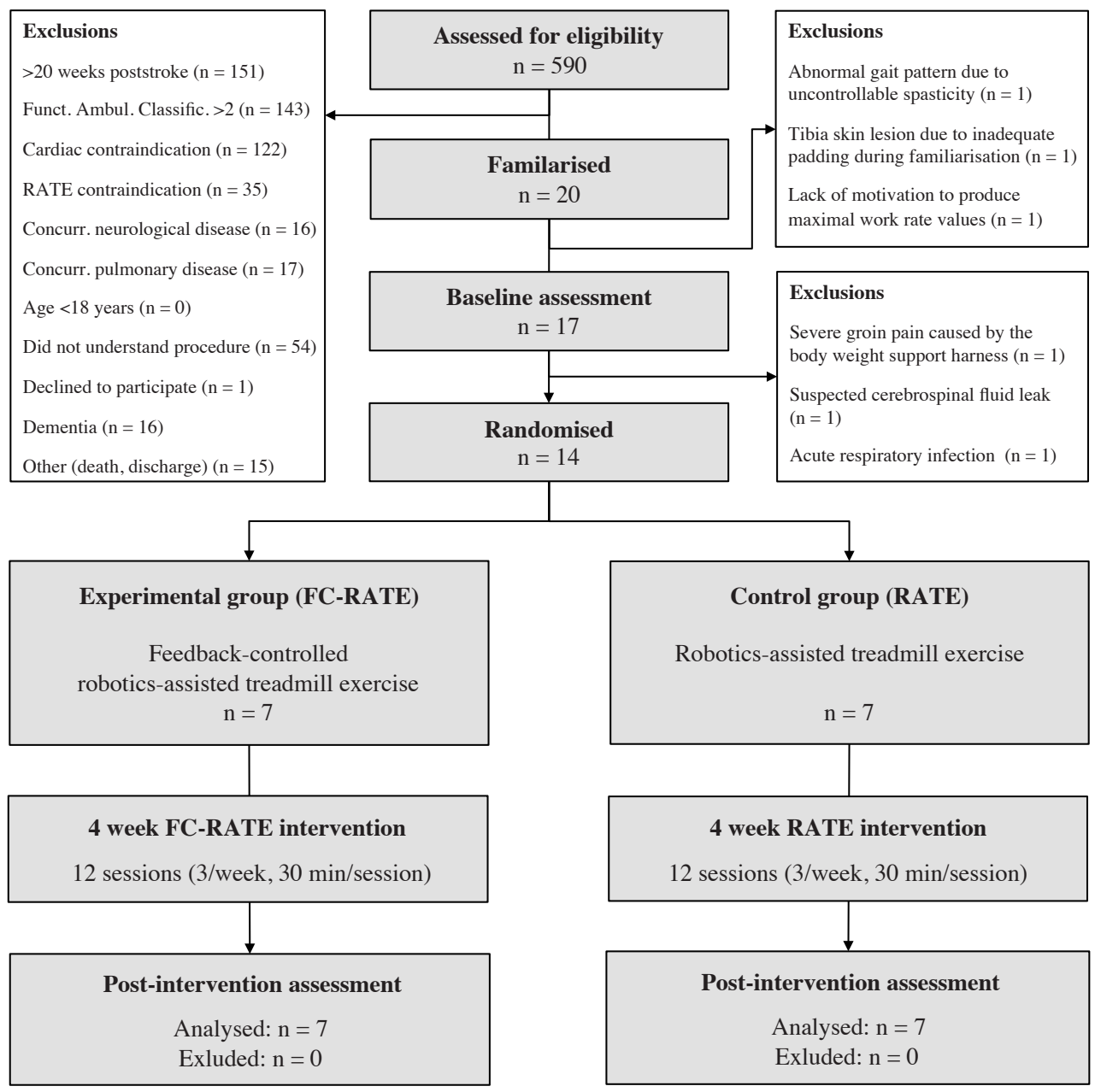

Figure 7.2: Study flow chart 


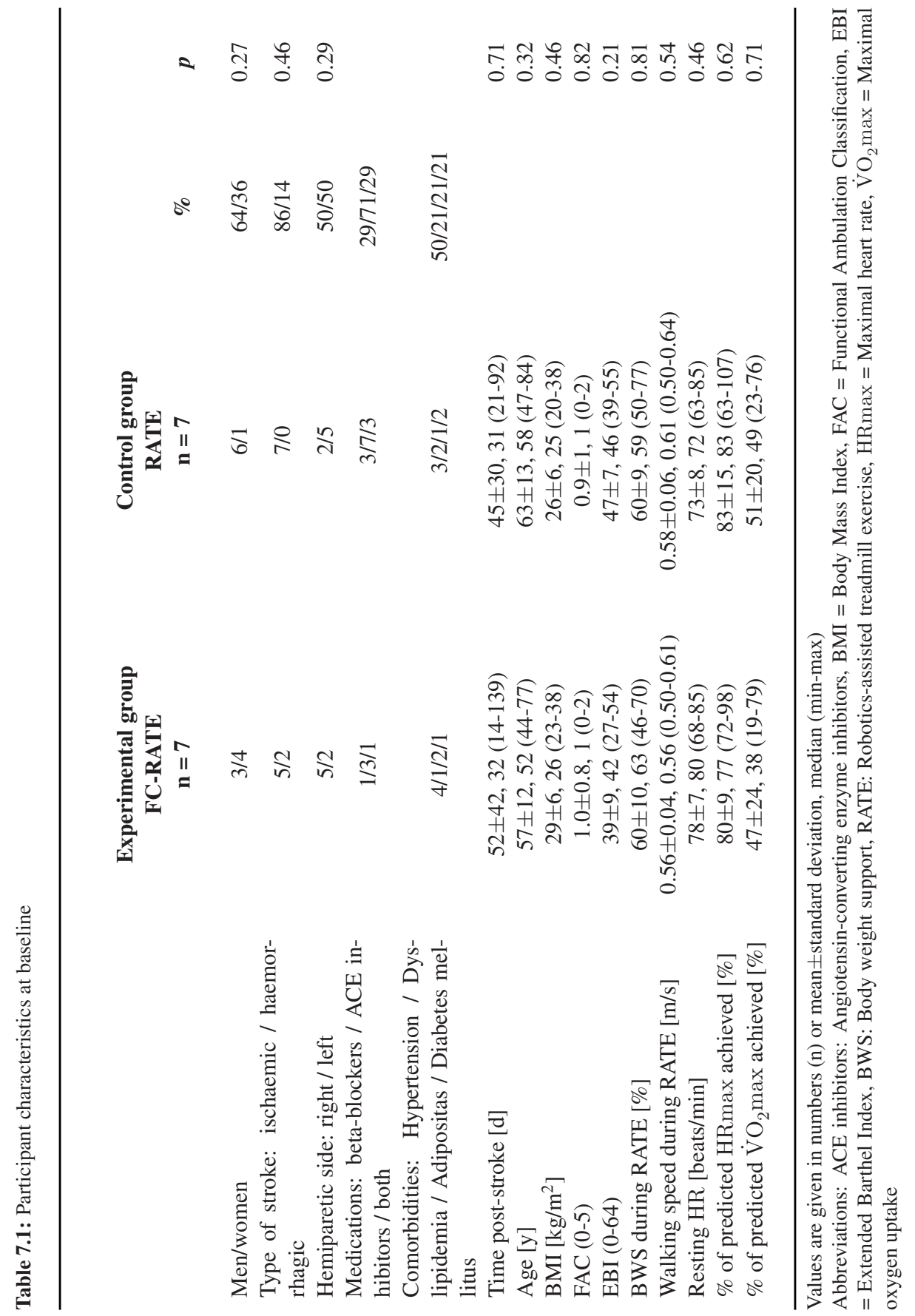




\section{Efficacy}

Peak cardiopulmonary performance showed no significant group-time interactions, but withingroup analyses revealed that both groups improved over time $\left(\mathrm{V}_{2}\right.$ peak absolute, $\mathrm{p}=0.02$, effect size (ES) 0.38; $\dot{\mathrm{V}}{ }_{2}$ peak relative, $\mathrm{p}=0.01$, $\mathrm{ES}=0.58 ; \dot{\mathrm{V}}_{\mathrm{E}}$ peak, $\mathrm{p}=0.001$, ES=0.49; RERpeak, $\mathrm{p}=0.001, \mathrm{ES}=0.82 ; \mathrm{O}_{2}$ pulse, $\mathrm{p}=0.02, \mathrm{ES}=0.29$ ) (Table 7.2). No other significant differences in cardiovascular performance parameters were found. Overall, absolute $\dot{\mathrm{V}}{ }_{2}$ peak increased from 1236 to $1477 \mathrm{~mL} / \mathrm{min}$ (48.2 to $57.6 \%$ of predicted absolute $\left.\dot{\mathrm{V}}{ }_{2} \max [56]\right)$, relative $\dot{\mathrm{V}}{ }_{2}$ peak from 14.6 to $17.7 \mathrm{~mL} / \mathrm{min} / \mathrm{kg}$ (45.8 to $55.7 \%$ of predicted relative $\dot{\mathrm{VO}}_{2} \max$ [56]), and HRpeak from 122 to 128 beats/min ( 80.7 to $84.7 \%$ of predicted maximal heart rate [52]).

Training intensity over the 4-week intervention period (12 sessions) was significantly different between the groups (heart rate, $\mathrm{p}=0.002$; heart rate reserve, $\mathrm{p}=0.001$ ). For the experimental group, heart rate was (mean \pm standard deviation) $110 \pm 8$ beats/min (95\%CI 103-116) and heart rate reserve was $40 \pm 3 \%$ (95\%CI 37-42). For the control group, heart rate was $83 \pm 13$ beats $/ \mathrm{min}$ (95\% CI 73-92) and heart rate reserve was $14 \pm 2 \%$ (95\% CI 12-16). The course of training intensity for both groups is shown in Figure 7.3.

\section{Feasibility}

The compliance to the intervention protocol was $100 \%$. All participants who completed the baseline assessment were able to complete the training protocol. However, several controllable and uncontrollable events led to an attrition rate of $30 \%$ during familiarisation and baseline assessment. Of the 6 participants who dropped out, only 2 gave reasons based on uncontrollable factors such as suspected cerebrospinal fluid leak and acute respiratory infection. The other 4 dropouts were caused by controllable factors. The gait pattern of 1 participant was disturbed by severe spasticity, which prevented a physiological gait pattern. One participant had a tibial skin lesion and another developed severe groin pain due to inappropriate padding. Furthermore, 1 participant was able to, but not motivated to, follow the target work rate, as described above. Although 2 cardiopulmonary exercise tests in 1 participant were considered as adverse events, no serious adverse events occurred during the study protocol (100\% clinical safety), and all data could be recorded continuously (100\% successful data acquisition). 


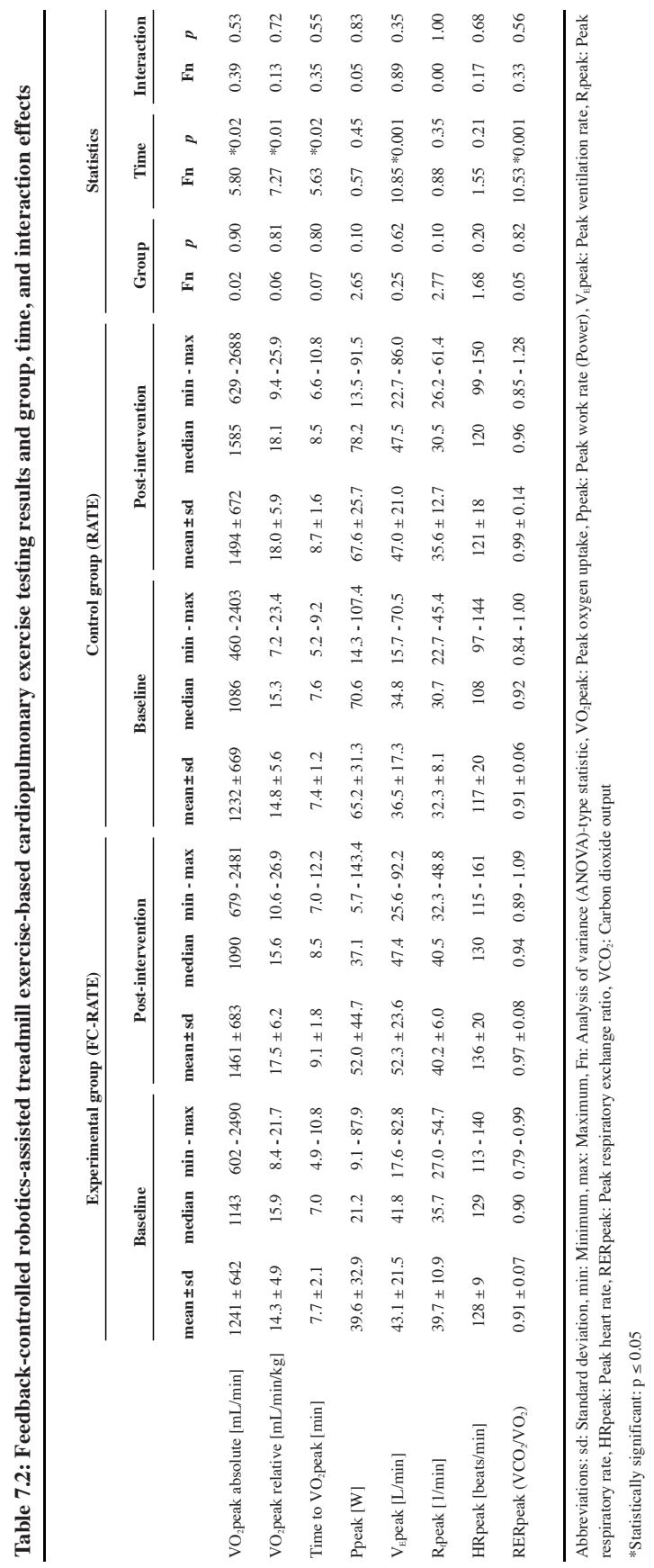




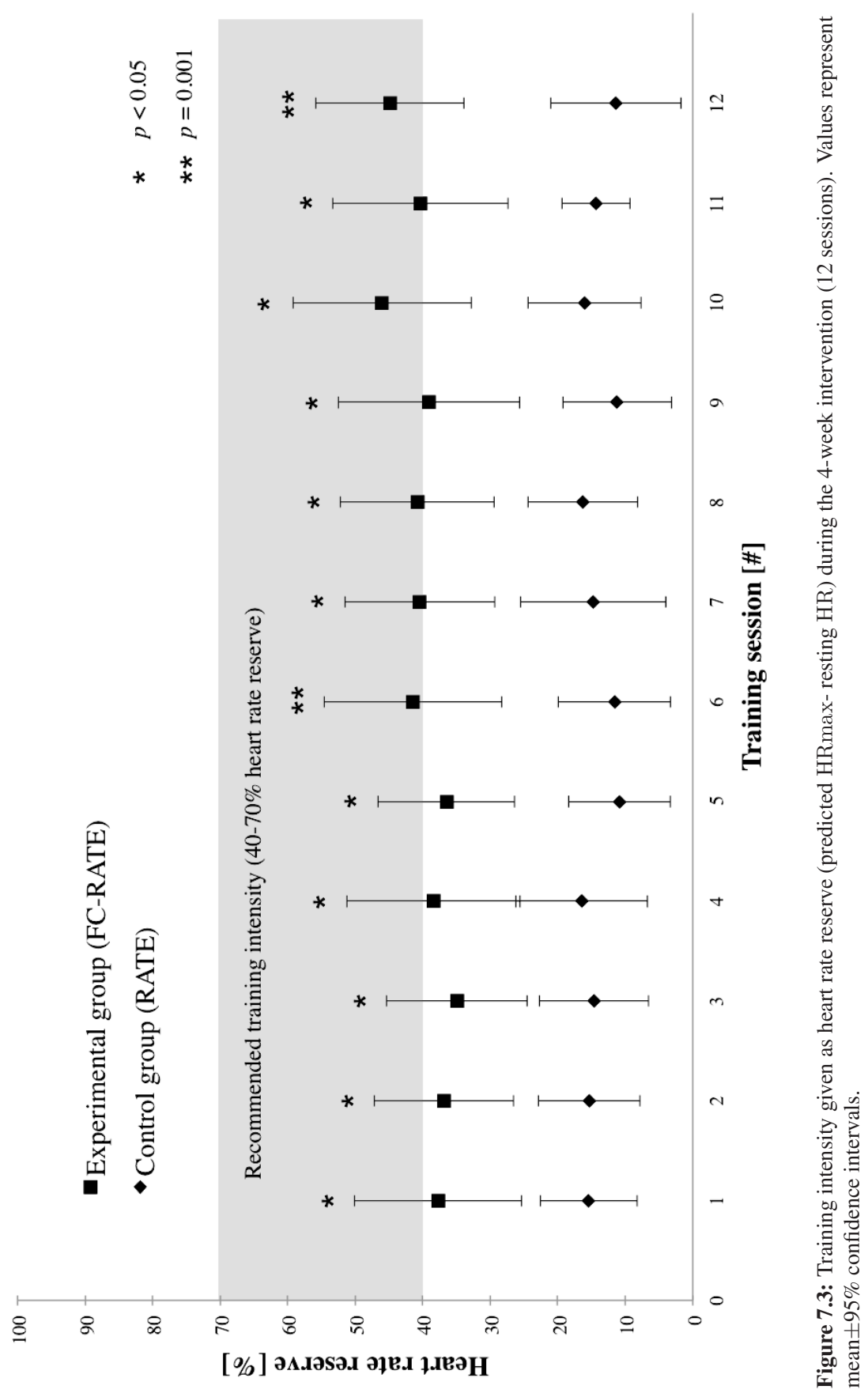




\section{Discussion}

This study aimed to carry out a preliminary investigation on efficacy and feasibility of FC-RATE for improving cardiovascular fitness in persons with severe motor impairments early after stroke. We hypothesised that FC-RATE would reach a substantially higher cardiovascular training intensity compared to conventional RATE in a clinical setting, thus resulting in significantly increased cardiovascular fitness following a 4-week FC-RATE intervention. In addition, we expected to affirm feasibility by achieving predefined criteria.

\section{Efficacy}

The results demonstrated substantial and significant overall increases in cardiovascular fitness, but no significant between-group differences when comparing FC-RATE with conventional RATE in a 4-week cardiovascular exercise intervention early after stroke. Although the FC-RATE concept achieved a significantly higher training intensity compared to conventional RATE, the difference between the two approaches was considered not to be clinically relevant. In detail, the experimental group did not consistently achieve the target range of 40-70\% heart rate reserve [37]. Sub-analyses revealed that only 3 of 7 participants in the experimental group (42\%) achieved at least $40 \%$ heart rate reserve during the intervention phase $(44 \%, 47 \%, 66 \%$ versus $28 \%, 29 \%$, 35\% heart rate reserve respectively). Thus, FC-RATE did not consistently achieve recommended intensity levels for cardiovascular training [37], which motivates improvement of the concept in its current technical state.

A major issue was the severely impaired status of the participants, which restricted exercise at higher target work rate values. This is underlined by the fact that the main reason for test termination during FC-RATE-based CPET was the inability to reach the target work rate due to generalised and/or leg fatigue. In addition, Ppeak in the experimental group was much lower at both time points compared to the control group, although not statistically different. This finding might have led to further limitations for the experimental group to achieve higher intensity levels. Even so, the high coordinative load of the concept in combination with the severely impaired status of the participants is a challenge. The period of time where participants could apply mechanical forces was probably too short, despite the slow walking speed of $0.57 \mathrm{~m} / \mathrm{s}$. Individuals generally tend to exercise using the unaffected side more dominantly, which leads to deviations from the predefined physiological gait pattern and further increases coordinative load. A further issue might be the low cardiovascular fitness status of the participants. The fact that all participants suffered from severe motor impairments, low cardiovascular fitness status and were not used to physical exercise 
training complicated the implementation of prolonged FC-RATE. The results clearly indicated a slow increase in mean training intensity over time for the experimental group (Figure 7.3).

However, it has been shown that even light to moderate exercise intensity is beneficial in deconditioned persons [57]. FC-RATE in its current form could, therefore, have potential for cardiovascular training. But the approach might have only limited power to promote significant between-group differences when compared to conventional RATE that has been shown to slightly increase exercise intensity $[34,35,36]$. Unfortunately, the present study protocol provided light training intensity for both groups and, thus, washed out potential between group-differences. It can be hypothesised that a longer intervention period and/or a comparison to conventional care (no RATE) would lead to significant differences in cardiovascular fitness, as FC-RATE has been shown to significantly increase exercise intensity to a moderate level.

A recent study that compared conventional RATE with standard care in a comparable sample found promising results favouring RATE within only 2 weeks [38]. Unfortunately, the authors did not report the effective training intensity (e.g. \% heart rate reserve), which led to difficulties for comparisons. They guided the training intensity for the RATE group by decreasing body weight support from $40 \%-0 \%$ and guidance force from $100 \%-10 \%$. While the sample in the present study was not able to walk with body weight support of $<40 \%$ due to severe motor limitations, their findings might be based on the comparison with a sedentary control group (conventional care), and the fact that the sample described was admitted earlier post-stroke. Previously, the time interval between stroke onset and intervention start as well as exercise intensity were shown to be predictive of training-induced gains in cardiovascular fitness [58].

The protocol presented here evaluated the minimal body weight support at baseline and adjustments during the intervention were only allowed within a range of $\pm 10 \%$ to maintain a physiological gait pattern. Although a further decrease in body weight support was not feasible due to the low motor function status of the included participants, the goal to substantially reduce body weight support remains important to optimally facilitate hemiparetic leg loading (activate relevant weight-bearing muscles) [59]. Advanced orthoses along with sophisticated controllers might provide solutions to enable unilateral assist-as-needed support during RATE in the future.

Both groups improved their $\dot{\mathrm{V}}{ }_{2}$ peak, the accepted criterion for cardiovascular fitness, after the 4-week intervention. The mean overall increase in relative $\dot{\mathrm{VO}_{2}}$ peak of $3.1 \mathrm{~mL} / \mathrm{min} / \mathrm{kg}$ $(+17.8 \%$, ES 0.58$)$ is nearly clinically meaningful, considering that $3.5 \mathrm{~mL} / \mathrm{min} / \mathrm{kg}$ has been associated with significantly fewer coronary events such as coronary artery disease [60] and a 12\% reduction in mortality in men with cardiac disease [61]. Individuals will generally profit from greater ability to engage in rehabilitation procedures at a lower percentage of their maximal exercise capac- 
ity. Considering the overall increase in $\dot{\mathrm{V}} \mathrm{O}_{2}$ peak of $+17.8 \%$ within 4 weeks, this is a promising result related to the spontaneous recovery of $\dot{\mathrm{VO}}_{2}$ peak of $16.9 \%$ in a 4-times longer period (20 weeks) reported in a comparable sample [62]. The significant time effect on $\dot{V}_{\text {Epeak }}(+19.9 \%)$, RERpeak $(+7.2 \%)$, and $\mathrm{O}_{2}$ pulse at $\dot{\mathrm{V}}{ }_{2}$ peak $(+11.6 \%)$ further underlines the improvement in exercise tolerance for both groups. Finally, the findings confirm the seriously compromised exercise capacity of individuals with severe impairments early after stroke, given the relative $\mathrm{V}_{2}$ peak values at post-intervention of $17.7 \pm 5.8 \mathrm{~mL} / \mathrm{min} / \mathrm{kg}$, which are $55.7 \%$ of predicted $\dot{\mathrm{VO}_{2}} \max$ [56].

\section{Feasibility}

Although the compliance to the intervention protocol was high, there was a dropout of 6 participants $(30 \%)$ during familiarisation and baseline assessment. Skin lesions and severe groin pain due to inappropriate padding are readily preventable by careful familiarisation and padding procedures; however, abnormal gait patterns due to spasticity and lack of motivation are more difficult to control. Extended familiarisation procedures, securing a careful padding of the limbs during all times, advances in harness design, and sophisticated work rate controllers are required to decrease attrition rates and improve motivation to elicit high target work rate levels in future trials.

The study demonstrated clinical safety and successful data acquisition. No serious adverse events occurred during FC-RATE-based CPET or during the intervention protocol. The strict eligibility criteria, designed to prevent adverse events in this pilot approach, led to the exclusion of a variety of individuals ( $97 \%$ of the initially screened population). For example, $21 \%$ were excluded due to cardiac contraindications for CPET. This large proportion of individuals with cardiac pathologies could profit from controlled cardiovascular exercise interventions. The number of individuals who cannot receive FC-RATE is rather small, i.e. only $6 \%$ had contraindications for RATE, which underlines the potential impact of the concept.

While the reliability of FC-RATE-based CPET has been demonstrated in a previous trial [41], the ability to evaluate true maximal exercise capacity using this novel approach needs to be tested. The fact that only $50 \%$ of the participants reached some criterion for maximal aerobic capacity suggests that the results on cardiovascular fitness presented here must be considered as submaximal overall. It could be that the guidelines postulated for healthy people and individuals with chronic stroke may not be realistic for determination of true exercise capacity in persons with severe motor limitations early after stroke. Further research in populations with severe motor impairments needs to establish valid criteria for maximal exercise capacity.

The clinical effort associated with the feedback-control approach presented in this study is comparable with conventional RATE. Additional measurements at baseline, such as the evaluation 
of minimal body weight support, can be easily implemented in clinical routine. As a minimum, 2 experienced therapists are needed to perform FC-RATE-based CPET, and 1 trained therapist can implement the training. Although previous work proposed a total-body recumbent stepper to implement cardiovascular exercise testing in persons with severe motor limitations [22, 23], and FC-RATE might be considered as a very elaborate and costly endeavour, this is the first study that presents a task-specific training device for assessment of cardiovascular fitness and guidance of exercise intensity early after severe stroke.

Overall, the concept presented in this study is deemed feasible with a need for major modifications. Considering the complexity of implementing the procedure in a sample with severe motor impairments early after stroke and the lack of effective cardiovascular intervention strategies, the findings presented here are of high clinical importance. The study revealed major issues associated with the customisation of RATE/FC-RATE and the consistent achievement of recommended intensity for prolonged training. Advances in body weight support systems and improved work rate controllers combined with appropriate visual and auditory feedback might provide solutions in the near future. For example, more degrees of freedom combined with individual joint control during the gait cycle would decrease body weight support to an absolute minimum, which in turn could increase exercise intensity in this population. Furthermore, the specific extensions of tasks (e.g. robotics-assisted stair climbing) could further increase cardiovascular stress and, hence, facilitate task-specific training.

\section{Limitations}

The major limitation of the current study is the small sample size, which may render the results underpowered. However, considering our pilot approach and the difficulty to implement an intensive cardiovascular exercise intervention in this early stage after severe stroke, the sample of 20 individuals at the outset was a realistic group size to obtain first estimates.

The results presented here could be partly explained by spontaneous recovery, in addition to the training intervention, and must therefore be interpreted with caution. Due to the fact that most of the exercise tests can be considered as submaximal, the overall increase in exercise capacity could have been influenced by the improved motor status of the participants.

The present study was not able to include a control group that received usual care only due to ethical considerations (all included participants would have received RATE as usual care).

Although demonstrating early beneficial effects, despite the short training duration that has been reported in previous trials $[8,38]$, the training volume and frequency in the current study was below recommended levels for cardiovascular exercise [57]. The length of the inpatient stay 
restricted the training volume to a 4-week period, and the weekly course of in-house rehabilitation limited the training frequency. Optimal training volume and frequency should theoretically reach 5 sessions/week for a minimum of 8 weeks [37].

As this was a pilot trial, outcomes beyond basic cardiovascular fitness measures such as vascular risk factors, motor function, gait pattern, cognition and well-being were not examined. Future trials need to establish deeper insight into the effects on cardiovascular health, motor recovery, cognition and quality of life.

\section{Conclusions}

Substantial and significant overall increases in the main cardiopulmonary performance parameters were observed in both groups, but there were no significant between-group differences when comparing FC-RATE and conventional RATE. FC-RATE significantly increased exercise intensity in persons with severe impairments early after stroke, but the recommended intensity levels for cardiovascular training were not consistently achieved. Future research should focus on the development of appropriate algorithms within advanced robotic systems to promote optimal cardiovascular stress. This study is an important step towards the implementation of effective cardiovascular exercise along with task-specific training early after severe stroke.

\section{References}

[1] M. Mackay-Lyons, A. McDonald, J. Matheson, G. Eskes, and M. A. Klus, "Dual effects of body-weight supported treadmill training on cardiovascular fitness and walking ability early after stroke: a randomized controlled trial," Neurorehabil Neural Repair, vol. 27, no. 7, pp. 644-53, 2013.

[2] S. A. Billinger, A. E. Mattlage, A. L. Ashenden, A. A. Lentz, G. Harter, and M. A. Rippee, "Aerobic exercise in subacute stroke improves cardiovascular health and physical performance," J Neurol Phys Ther, vol. 36, no. 4, pp. 159-65, 2012.

[3] I. T. da Cunha, P. A. Lim, H. Qureshy, H. Henson, T. Monga, and E. J. Protas, "Gait outcomes after acute stroke rehabilitation with supported treadmill ambulation training: A randomized controlled pilot study," Archives of Physical Medicine and Rehabilitation, vol. 83, no. 9, pp. 1258-1265, 2002.

[4] P. Duncan, S. Studenski, L. Richards, S. Gollub, S. M. Lai, D. Reker, S. Perera, J. Yates, V. Koch, S. Rigler, and D. Johnson, "Randomized clinical trial of therapeutic exercise in subacute stroke," Stroke, vol. 34, no. 9, pp. 2173-2180, 2003.

[5] H. J. Eich, H. Mach, C. Werner, and S. Hesse, "Aerobic treadmill plus bobath walking training improves walking in subacute stroke: a randomized controlled trial," Clinical Rehabilitation, vol. 18, no. 6, pp. 640-651, 2004.

[6] M. Katz-Leurer, M. Shochina, E. Carmeli, and Y. Friedlander, "The influence of early aerobic training on the functional capacity in patients with cerebrovascular accident at the subacute stage," Archives of Physical Medicine and Rehabilitation, vol. 84, no. 11, pp. 1609-1614, 2003. 
[7] J. C. Outermans, R. P. S. van Peppen, H. Wittink, T. Takken, and G. Kwakkel, "Effects of a high-intensity taskoriented training on gait performance early after stroke: a pilot study," Clinical Rehabilitation, vol. 24, no. 11, pp. 979-987, 2010.

[8] A. Tang, K. M. Sibley, S. G. Thomas, M. T. Bayley, D. Richardson, W. E. McIlroy, and D. Brooks, "Effects of an aerobic exercise program on aerobic capacity, spatiotemporal gait parameters, and functional capacity in subacute stroke," Neurorehabilitation and Neural Repair, vol. 23, no. 4, pp. 398-406, 2009.

[9] A. Tang, S. Marzolini, P. Oh, W. E. McIlroy, and D. Brooks, "Feasibility and effects of adapted cardiac rehabilitation after stroke: a prospective trial," BMC Neurology, vol. 10, p. 40, 2010.

[10] A. Letombe, C. Cornille, H. Delahaye, A. Khaled, O. Morice, A. Tomaszewski, and N. Olivier, "Early poststroke physical conditioning in hemiplegic patients: A preliminary study," Annals of Physical Rehabilitation Medicine, vol. 53, no. 10, pp. 632-642, 2010.

[11] A. Toledano-Zarhi, D. Tanne, E. Carmeli, and M. Katz-Leurer, "Feasibility, safety and efficacy of an early aerobic rehabilitation program for patients after minor ischemic stroke: A pilot randomized controlled trial," Neurorehabilitation, vol. 28, no. 2, pp. 85-90, 2011.

[12] O. Stoller, E. D. de Bruin, R. H. Knols, and K. J. Hunt, "Effects of cardiovascular exercise early after stroke: systematic review and meta-analysis," BMC Neurology, vol. 12, no. 1, p. 45, 2012.

[13] C. Globas, C. Becker, J. Cerny, J. M. Lam, U. Lindemann, L. W. Forrester, R. F. Macko, and A. R. Luft, “Chronic stroke survivors benefit from high-intensity aerobic treadmill exercise: a randomized control trial," Neurorehabil Neural Repair, vol. 26, no. 1, pp. 85-95, 2012.

[14] J. R. Jorgensen, D. T. Bech-Pedersen, P. Zeeman, J. Sorensen, L. L. Andersen, and M. Schonberger, "Effect of intensive outpatient physical training on gait performance and cardiovascular health in people with hemiparesis after stroke," Physical Therapy, vol. 90, no. 4, pp. 527-537, 2010.

[15] R. F. Macko, F. M. Ivey, L. W. Forrester, D. Hanley, J. D. Sorkin, L. I. Katzel, K. H. Silver, and A. P. Goldberg, "Treadmill exercise rehabilitation improves ambulatory function and cardiovascular fitness in patients with chronic stroke - a randomized, controlled trial," Stroke, vol. 36, no. 10, pp. 2206-2211, 2005.

[16] K. Potempa, M. Lopez, L. T. Braun, J. P. Szidon, L. Fogg, and T. Tincknell, "Physiological outcomes of aerobic exercise training in hemiparetic stroke patients," Stroke, vol. 26, no. 1, pp. 101-105, 1995.

[17] M. Y. C. Pang, J. J. Eng, A. S. Dawson, and S. Gylfadottir, "The use of aerobic exercise training in improving aerobic capacity in individuals with stroke: a meta-analysis," Clinical Rehabilitation, vol. 20, no. 2, pp. 97-111, 2006.

[18] F. M. Ivey, A. S. Ryan, C. E. Hafer-Macko, and R. F. Macko, "Improved cerebral vasomotor reactivity after exercise training in hemiparetic stroke survivors," Stroke, vol. 42, no. 7, pp. 1994-2000, 2011.

[19] A. R. Luft, R. F. Macko, L. W. Forrester, F. Villagra, F. Ivey, J. D. Sorkin, J. Whitall, S. McCombe-Waller, L. Katzel, A. P. Goldberg, and D. F. Hanley, "Treadmill exercise activates subcortical neural networks and improves walking after stroke: a randomized controlled trial,” Stroke, vol. 39, no. 12, pp. 3341-50, 2008.

[20] M. S. El-Tamawy, F. Abd-Allah, S. M. Ahmed, M. H. Darwish, and H. A. Khalifa, "Aerobic exercises enhance cognitive functions and brain derived neurotrophic factor in ischemic stroke patients," NeuroRehabilitation, vol. 34, no. 1, pp. 209-13, 2014.

[21] B. M. Quaney, L. A. Boyd, J. M. McDowd, L. H. Zahner, J. H. He, M. S. Mayo, and R. F. Macko, "Aerobic exercise improves cognition and motor function poststroke," Neurorehabilitation and Neural Repair, vol. 23, no. 9, pp. 879-885, 2009.

[22] L. J. Durstine, G. Moore, P. Painter, and S. Roberts, ACSM's Exercise Management for Persons with Chronic Diseases and Disabilities. Champaign, IL: Human Kinetics, 3rd ed., 2009. 
[23] S. A. Billinger, B. Y. Tseng, and P. M. Kluding, "Modified total-body recumbent stepper exercise test for assessing peak oxygen consumption in people with chronic stroke," Physical Therapy, vol. 88, no. 10, pp. 1188-95, 2008 .

[24] P. Langhorne, F. Coupar, and A. Pollock, "Motor recovery after stroke: a systematic review," Lancet Neurology, vol. 8, no. 8, pp. 741-754, 2009.

[25] B. French, L. H. Thomas, M. J. Leathley, C. J. Sutton, J. McAdam, A. Forster, P. Langhorne, C. I. M. Price, A. Walker, and C. L. Watkins, "Repetitive task training for improving functional ability after stroke," Cochrane Database of Systematic Reviews, no. 4, p. CD006073, 2007.

[26] S. Hesse, M. Konrad, and D. Uhlenbrock, “Treadmill walking with partial body weight support versus floor walking in hemiparetic subjects," Arch Phys Med Rehabil, vol. 80, no. 4, pp. 421-7, 1999.

[27] A. Danielsson and K. S. Sunnerhagen, "Oxygen consumption during treadmill walking with and without body weight support in patients with hemiparesis after stroke and in healthy subjects," Archives of Physical Medicine Rehabilitation, vol. 81, no. 7, pp. 953-957, 2000.

[28] M. MacKay, L. Makrides, and S. Speth, "Effect of 15exercise capacity of adults without impairments," Physical Therapy, vol. 81, no. 11, pp. 1790-1800, 2001.

[29] T. G. Hornby, D. D. Campbell, J. H. Kahn, T. Demott, J. L. Moore, and H. R. Roth, "Enhanced gait-related improvements after therapist- versus robotic-assisted locomotor training in subjects with chronic stroke: a randomized controlled study," Stroke; a Journal of Cerebral Circulation, vol. 39, no. 6, pp. 1786-1792, 2008.

[30] B. Husemann, F. Müller, C. Krewer, S. Heller, and E. Koenig, "Effects of locomotion training with assistance of a robot-driven gait orthosis in hemiparetic patients after stroke: a randomized controlled pilot study," Stroke; a Journal of Cerebral Circulation, vol. 38, no. 2, pp. 349-354, 2007.

[31] C. Werner, S. von Frankenberg, T. Treig, M. Konrad, and S. Hesse, "Treadmill training with partial body weight support and an electromechanical gait trainer for restoration of gait in subacute stroke patients: A randomized crossover study," Stroke, vol. 33, no. 12, pp. 2895-2901, 2002.

[32] D. J. Reinkensmeyer, O. Akoner, D. P. Ferris, and K. E. Gordon, "Slacking by the human motor system: computational models and implications for robotic orthoses," Conf Proc IEEE Eng Med Biol Soc, vol. 2009, pp. 2129-32, 2009.

[33] J. F. Israel, D. D. Campbell, J. H. Kahn, and T. G. Hornby, "Metabolic costs and muscle activity patterns during robotic- and therapist-assisted treadmill walking in individuals with incomplete spinal cord injury," Physical Therapy, vol. 86, no. 11, pp. 1466-1478, 2006.

[34] C. Krewer, F. Müller, B. Husemann, S. Heller, J. Quintern, and E. Koenig, "The influence of different lokomat walking conditions on the energy expenditure of hemiparetic patients and healthy subjects," Gait Posture, vol. 26, no. 3, pp. 372-377, 2007.

[35] M. P. van Nunen, K. H. Gerrits, A. de Haan, and T. W. Janssen, "Exercise intensity of robot-assisted walking versus overground walking in nonambulatory stroke patients," J Rehabil Res Dev, vol. 49, no. 10, pp. 1537-46, 2012.

[36] O. Stoller, E. D. de Bruin, M. Schindelholz, C. Schuster, R. A. de Bie, and K. J. Hunt, "Evaluation of exercise capacity after severe stroke using robotics-assisted treadmill exercise: A proof-of-concept study," Technology and Health Care, vol. 21, no. 2, pp. 157-166, 2013.

[37] S. A. Billinger, R. Arena, J. Bernhardt, J. J. Eng, B. A. Franklin, C. M. Johnson, M. MacKay-Lyons, R. F. Macko, G. E. Mead, E. J. Roth, M. Shaughnessy, A. Tang, C. American Heart Association Stroke, C. Council on, N. Stroke, L. Council on, H. Cardiometabolic, E. Council on, Prevention, and C. Council on Clinical, "Physical activity and exercise recommendations for stroke survivors: a statement for healthcare professionals from the american heart association/american stroke association," Stroke, vol. 45, no. 8, pp. 2532-53, 2014. 
[38] W. H. Chang, M. S. Kim, J. P. Huh, P. K. W. Lee, and Y. H. Kim, "Effects of robot-assisted gait training on cardiopulmonary fitness in subacute stroke patients: A randomized controlled study," Neurorehabilitation and Neural Repair, vol. 26, no. 4, pp. 318-324, 2012.

[39] K. J. Hunt, L. P. Jack, A. Pennycott, C. Perret, M. Baumberger, and T. H. Kakebeeke, "Control of work ratedriven exercise facilitates cardiopulmonary training and assessment during robot-assisted gait in incomplete spinal cord injury," Biomedical Signal Processing and Control, vol. 3, no. 1, pp. 19-28, 2008.

[40] O. Stoller, M. Schindelholz, L. Bichsel, C. Schuster, R. A. de Bie, E. D. de Bruin, and K. J. Hunt, "Feedbackcontrolled robotics-assisted treadmill exercise to assess and influence aerobic capacity early after stroke: a proofof-concept study," Disabil Rehabil Assist Technol, vol. 9, no. 4, pp. 271-278, 2014.

[41] O. Stoller, E. D. de Bruin, M. Schindelholz, C. Schuster-Amft, R. A. de Bie, and K. J. Hunt, "Cardiopulmonary exercise testing early after stroke using feedback-controlled robotics-assisted treadmill exercise: test-retest reliability and repeatability," Journal of Neuroengineering and Rehabilitation, vol. 11, p. 145, 2014.

[42] M. Campbell, R. Fitzpatrick, A. Haines, A. L. Kinmonth, P. Sandercock, D. Spiegelhalter, and P. Tyrer, "Framework for design and evaluation of complex interventions to improve health," BMJ, vol. 321, no. 7262, pp. 694-6, 2000 .

[43] O. Stoller, E. D. de Bruin, C. Schuster-Amft, M. Schindelholz, R. A. de Bie, and K. J. Hunt, "Cardiovascular rehabilitation soon after stroke using feedback-controlled robotics-assisted treadmill exercise: study protocol of a randomised controlled pilot trial," Trials, vol. 14, no. 1, p. 304, 2013.

[44] W. R. Thompson, N. F. Gordon, and L. S. Pescatello, American College of Sports Medicine. Guidelines for Exercise Testing and Prescription. Philadelphia: Lippincott Williams Wilkins, eight ed., 2010.

[45] L. Thabane, J. Ma, R. Chu, J. Cheng, A. Ismaila, L. P. Rios, R. Robson, M. Thabane, L. Giangregorio, and C. H. Goldsmith, "A tutorial on pilot studies: the what, why and how," BMC Med Res Methodol, vol. 10, p. 1, 2010.

[46] M. Schindelholz, O. Stoller, and K. J. Hunt, "A software module for cardiovascular rehabilitation in roboticsassisted treadmill exercise,” Biomedical Signal Processing and Control, vol. 10, pp. 296-307, 2014.

[47] “Ats/accp statement on cardiopulmonary exercise testing," American Journal of Respiratory and Critical Care Medicine, vol. 167, pp. 211-277, 2003.

[48] G. A. Borg, "Perceived exertion," Exerc Sport Sci Rev, vol. 2, pp. 131-153, 1974.

[49] R. A. Robergs, D. Dwyer, and T. Astorino, "Recommendations for improved data processing from expired gas analysis indirect calorimetry," Sports Medicine, vol. 40, no. 2, pp. 95-111, 2010.

[50] D. C. Poole, D. P. Wilkerson, and A. M. Jones, "Validity of criteria for establishing maximal o2 uptake during ramp exercise tests," Eur J Appl Physiol, vol. 102, no. 4, pp. 403-10, 2008.

[51] W. L. Beaver, K. Wasserman, and B. J. Whipp, "A new method for detecting anaerobic threshold by gasexchange,” Journal of Applied Physiology, vol. 60, no. 6, pp. 2020-2027, 1986.

[52] H. Tanaka, K. D. Monahan, and D. R. Seals, "Age-predicted maximal heart rate revisited," Journal of the American College of Cardiology, vol. 37, no. 1, pp. 153-156, 2001.

[53] P. A. Tesch, "Exercise performance and beta-blockade," Sports Medicine, vol. 2, no. 6, pp. 389-412, 1985.

[54] J. Karvonen and T. Vuorimaa, "Heart rate and exercise intensity during sports activities. practical application," Sports Med, vol. 5, no. 5, pp. 303-11, 1988.

[55] E. Brunner, S. Domhof, and F. Langer, Nonparametric analysis of longitudinal data in factorial experiments, vol. 406. Wiley-Interscience, 2002. 
[56] R. Jurca, A. S. Jackson, M. J. LaMonte, J. R. Morrow, S. N. Blair, N. J. Wareham, W. L. Haskell, W. van Mechelen, T. S. Church, J. M. Jakicic, and R. Laukkanen, "Assessing cardiorespiratory fitness without performing exercise testing," American Journal of Preventive Medicine, vol. 29, no. 3, pp. 185-193, 2005.

[57] C. E. Garber, B. Blissmer, M. R. Deschenes, B. A. Franklin, M. J. Lamonte, I. M. Lee, D. C. Nieman, D. P. Swain, and M. American College of Sports, "American college of sports medicine position stand. quantity and quality of exercise for developing and maintaining cardiorespiratory, musculoskeletal, and neuromotor fitness in apparently healthy adults: guidance for prescribing exercise," Med Sci Sports Exerc, vol. 43, no. 7, pp. 1334-59, 2011.

[58] J. M. Lam, C. Globas, J. Cerny, B. Hertler, K. Uludag, L. W. Forrester, R. F. Macko, D. F. Hanley, C. Becker, and A. R. Luft, "Predictors of response to treadmill exercise in stroke survivors," Neurorehabil Neural Repair, vol. 24 , no. 6 , pp. 567-74, 2010.

[59] S. Hesse, "Treadmill training with partial body weight support after stroke: a review," NeuroRehabilitation, vol. 23, no. 1, pp. 55-65, 2008.

[60] R. Hambrecht, C. Walther, S. Mobius-Winkler, S. Gielen, A. Linke, K. Conradi, S. Erbs, R. Kluge, K. Kendziorra, O. Sabri, P. Sick, and G. Schuler, "Percutaneous coronary angioplasty compared with exercise training in patients with stable coronary artery disease: a randomized trial," Circulation, vol. 109, no. 11, pp. 1371-8, 2004.

[61] J. Myers, M. Prakash, V. Froelicher, D. Do, S. Partington, and J. E. Atwood, "Exercise capacity and mortality among men referred for exercise testing," New England Journal of Medicine, vol. 346, no. 11, pp. 793-801, 2002 .

[62] M. J. MacKay-Lyons and L. Makrides, "Longitudinal changes in exercise capacity after stroke," Archives of Physical Medicine and Rehabilitation, vol. 85, no. 10, pp. 1608-1612, 2004. 



\section{Chapter 8}

\section{General discussion}


Regular cardiovascular exercise can improve overall health status, modify risk factors for cardiovascular disease, and improve exercise capacity, which is of high importance during stroke rehabilitation. To date, rehabilitation strategies to improve cardiovascular fitness in severely motor impaired individuals are inefficient and do not embody principles for motor learning (i.e. task-specific exercise). Considering the relatively short intervention window during stroke recovery, advanced intervention approaches (i.e. robotics-assisted treadmill exercise) might have high potential to facilitate a wide spectrum of key elements (e.g. cardiovascular stress, task-specific training) to attain highly effective stroke rehabilitation.

The driving hypothesis of this dissertation was that the implementation of cardiovascular exercise early after stroke will positively influence cardiovascular health, which might further facilitate overall recovery after stroke. Here, robotics-assisted treadmill exercise might have the potential to introduce cardiovascular rehabilitation into the early stages after stroke, where deconditioning and severe motor impairments are highly prevalent. This novel concept would provide a relevant methodology for exercise testing and cardiovascular exercise prescription for stroke survivors with severe motor limitations.

The aims of this thesis were: (1) to review knowledge about the effects of cardiovascular exercise early after stroke, (2) to implement a robotic-based strategy to accomplish recent standards for exercise testing and prescription for severely motor impaired stroke survivors, and (3) to systematically evaluate the concept within a clinical setting.

This chapter discusses the main research findings, presents limitations and methodological considerations, provides recommendations for future research and clinical practice, and completes with the main original contributions of the thesis. 


\section{Main findings}

\section{Cardiovascular exercise early after stroke}

The systematic review and meta-analysis provided an overview of the currently available evidence on cardiovascular exercise in the early stages after stroke (CHAPTER 2, [1]). The results provide robust evidence that subacute stroke survivors benefit from cardiovascular exercise protocols to improve $\mathrm{V}_{2}$ peak and walking endurance. Despite the fact that some of the included studies revealed moderate to large effect sizes (ES) for improving gait speed, it remains unclear whether cardiovascular exercise has an impact on ambulatory function. The inconsistent use of exercise protocols (e.g. leg cycle ergometry, treadmill exercise) might have biased these outcomes due to the training of the function itself, while treadmill exercise might have led to larger improvements in functional walking capacity compared to leg cycle ergometry. Further measures of motor recovery, functional ability and quality of life did not consistently improve, and large heterogeneity in the applied outcome measurements was found that restricted further synthesises of the results. While recent research in chronic stroke reported preliminary evidence for the effects of cardiovascular exercise on cerebral blood flow [2], neuroplasticity [3], and cognition [4, 5, 6, 7, 8], none of the studies included reported variables related to these outcomes in subacute stroke. As a result, the efficacy of cardiovascular exercise early after stroke on motor recovery, functional ability, quality of life, cerebral blood flow, neuroplasticity and cognition remains unknown.

Cardiovascular exercise has been shown to be safe in subacute stroke care. The incidence rate of adverse events such as recurrent stroke was comparable to the usual recurrent stroke risk. However, the strict exclusion criteria reported have excluded stroke survivors with severe cardiac disease, and the applicability of cardiovascular exercise in the acute period after stroke $(<7$ days post event) remains unclear. Therefore, the safety of cardiovascular exercise early after stroke has not yet been fully assessed.

To date, leg cycle ergometry is the most appropriate and valid cardiopulmonary exercise testing (CPET) method for assessment of exercise capacity in individuals early after stroke. None of the reviewed studies reported major adverse events, which can be regarded as an encouraging finding since it raises the possibility of starting CPET and cardiovascular exercise training in the very early period after stroke.

Recent research included mildly to moderately impaired stroke survivors only. These individuals are able to perform CPET and cardiovascular exercise interventions on established devices such as semi-recumbent leg cycle ergometers, conventional leg cycle ergometers, or treadmills. 
There is a lack of evidence for the severely motor impaired population after stroke due to unique challenges in motor control. New concepts for assessment and improvement of exercise capacity in severely disabled individuals are sought to explore potential effects of early cardiovascular exercise administration.

\section{Implementation of robotics-assisted treadmill technology}

A potential approach to overcome severe motor limitations while facilitating cardiovascular stress is the introduction of robotics-assisted treadmill exercise (RATE). A proof-of-concept study that used a control strategy based on the reduction of body weight support (BWS) demonstrated the feasibility of provoking substantial cardiovascular stress, however, the step increases were not distinct enough to accurately calculate cardiopulmonary performance parameters (i.e. oxygen uptake kinetics $(\tau)$ ) (CHAPTER 3, [9]). A further control strategy based on guidance force (GF) reduction was not appropriate to elicit peak cardiovascular responses for the assessment of exercise capacity. These findings are in line with previous studies that have shown that conventional RATE is not passive, and the control of BWS and GF has only a minor influence on cardiovascular responses $[10,11]$. The major issue of both strategies was to provide an appropriate challenge level while simultaneously supporting the severe motor impairments of the individuals. Sudden step increases based on BWS reduction during constant load testing (CLT), and incremental exercise testing (IET) based on GF reduction, might challenge the motor system that provokes some metabolic responses. Conventional control strategies are not sophisticated enough to adapt to individual motor impairments to provoke substantial cardiovascular responses for the assessment of exercise capacity and achievement of recommended exercise intensity levels for cardiovascular rehabilitation.

While previous studies on individuals with spinal cord jury showed similar cardiovascular stress during active walking within a robotics-assisted treadmill compared to conventional treadmill exercise $[12,13]$, the focus shifted to the control of active participation. A novel assessment protocol has been implemented to estimate key cardiopulmonary performance parameters during RATE (CHAPTER 4, [14]). This incorporates a biofeedback mechanism, which allows individuals to maximise their voluntary effort and associated cardiovascular stress during RATE. A further proof-of-concept study, evaluating the concept of feedback-controlled robotics-assisted treadmill exercise (FC-RATE), demonstrated feasibility for guidance and assessment of exercise capacity in severely motor impaired individuals early after stroke. Results showed reasonable cardiopulmonary performance parameters compared to previous studies on conventional CPET early after stroke $[15,16,17,18]$. The approach presented acceptable time constants $(\tau)$ for oxygen uptake kinetics for the passive to active step response, which were comparable to previous studies [19]. 
This might be of importance for the assessment of exercise capacity in high-risk populations, where peak performance cannot be provoked. Individuals also demonstrated high accuracy of work rate tracking, which means that subjects were able to follow the target work rate during walking on the robotics-assisted treadmill. The FC-RATE concept has been taken for further consideration as a potential method for assessment of exercise capacity and guidance of exercise intensity in severely motor impaired individuals early after stroke. The question then has arisen, how reliable and repeatable these parameters are.

\section{Assessment of exercise capacity using FC-RATE-based CPET}

Further research investigated the reliability and the repeatability of FC-RATE-based CPET for assessment of exercise capacity early after stroke (CHAPTER 5, [20]). Peak cardiopulmonary performance parameters were slightly higher compared to previous studies using mostly leg-cycle ergometry for CPET. This finding might be based on to the introduction of treadmill exercise that has previously confirmed higher peak values compared to leg cycle ergometry protocols $[15,17,18,21,22,23]$. The FC-RATE approach might have led to the recruitment of additional muscle mass compared to conventional procedures. Although the results yielded high peak values, the question of whether the concept is able to meet traditional criteria for true maximal exercise capacity remains. Based on the findings regarding the achievement of peak exercise capacity, we hypothesise that the guidelines postulated for healthy and chronic stroke populations so far may not be realistic for determination of true exercise capacity in early stages after stroke. This view is supported by other researchers [15]. The definition of indices for peak exercise capacity early after stroke must be part of future research.

FC-RATE-based CPET confirmed good to excellent relative reliability and acceptable absolute reliability for the major peak cardiopulmonary performance parameters during IET. While our findings on reliability are in line with previous studies [15, 16, 24, 25, 26], absolute reliability was lower compared to previous studies using leg cycle ergometry. Regarding repeatability, studies in healthy subjects and individuals with cardiac or respiratory disease have shown low coefficients of variation (CoV) $[27,28,29,30]$. Our results yielded considerably higher CoV for the major cardiopulmonary parameters. This finding might be based on the complex approach presented here. The extensive setup and the coordinative challenge to achieve the target work rate might have increased the variability. It should be noted that only limited evidence is available so far on reliability and repeatability of CPET in the early post-stroke period, thus, our findings must be rated as initial data on a novel approach where comparisons are a difficulty.

CLT revealed high mean differences and insufficient test-retest reliability for all variables 
studied. Time constants of oxygen uptake kinetics $(\tau)$ could not be evaluated due to the inherent noisiness of the breath-by-breath data and the consequent poor signal-to-noise ratio, which led to low goodness of fit. The CLT approach presented here seems to be incapable to provide consistent outcome values for the transition phases. Sudden onset of changes in BWS and walking pattern seem to have a strong impact on individual performance levels during conventional RATE, which restricts valid measures. Further development in control strategies might be able to decrease the variability during the transition phases.

\section{Efficacy of FC-RATE for cardiovascular rehabilitation early after stroke}

The efficacy and the clinical feasibility of FC-RATE for improvement of exercise capacity early after stroke has been established in a randomised controlled pilot trial (CHAPTER 6,7 [31, 32]). Results indicated that cardiovascular exercise intensity can be significantly increased in severely motor impaired individuals early after stoke using FC-RATE, but the method was not superior to conventional RATE to improve cardiovascular fitness within a 4-weeks intervention. We hypothesise that the difference in training intensity, duration, and frequency between the two groups was not high enough to prove the superiority of FC-RATE over conventional RATE. The control group might have profited from regular moderate cardiovascular exercise, which has shown to be effective in deconditioned persons [33] where the severe motor limitations in the early intervention stages might have provoked some cardiovascular response (as shown in CHAPTER 3) to facilitate improvements in cardiovascular fitness.

A recent study that compared progressive RATE with conventional care in a comparable sample with subacute stroke found promising results favouring RATE within a 2-week intervention period [34]. The training intensity for the RATE group was guided by decreasing BWS from 40\%$0 \%$ and GF from $100 \%-10 \%$. While our sample was not able to walk with BWS $<40 \%$ due to severe motor limitations, their findings might be based on the high intensity level reached during conventional RATE and the comparison to a sedentary control group (conventional care). This supports our hypothesis that the training intensity of our control group was probably too high. However, the authors did not report training intensity (e.g. heart rate) that leads to difficulties in comparing the findings regarding the training intensity. Furthermore, the question regarding how the BWS and the GF were decreased to such a low level remain questionable due to the severe motor limitations of the individuals included.

Although we found no between-group differences after a 4-weeks intervention, both groups improved their $\dot{\mathrm{VO}_{2}}$ peak, the accepted criterion for cardiovascular fitness. The mean increase in relative $\mathrm{VO}_{2}$ peak of $3.1 \mathrm{~mL} / \mathrm{min} / \mathrm{kg}(+17.8 \%$, effect size (ES) 0.58$)$ is nearly clinically meaning- 
ful, considering that $3.5 \mathrm{~mL} / \mathrm{min} / \mathrm{kg}$ has been associated with significantly fewer clinical events in coronary artery disease [35] and a $12 \%$ reduction in mortality among men with cardiac disease [36]. Subjects generally will profit from greater ability to engage in rehabilitation procedures at a lower percentage of their maximal exercise capacity. However, Ppeak, an important criterion for exercise capacity has shown no significant time effects, which means that subjects did not improve their maximal work rate values within the 4-weeks intervention period. Even so, the gas exchange threshold (GET), an important value regarding the onset of the anaerobic metabolism, showed no significant differences at post-intervention assessments. These findings might be explained by the severe motor impairments, the high coordinative demand of the approach, and the short intervention period of 4-weeks. Larger training duration up to 6 months might be able to improve coordinative skills, which may increase maximal work rate values, and induce physiological changes within the muscular system to modulate the metabolic state.

In conclusion, our findings close the gap between the lack of an effective assessment and intervention strategy for cardiovascular rehabilitation in severely impaired individuals early after stroke. FC-RATE provides reliable data for peak exercise capacity and is able to significantly increase the training intensity in a clinical setting. The approach opens new perspectives in exploring the effects of early cardiovascular exercise admission in severely impaired populations.

\section{Limitations and methodological considerations}

\section{Study sample}

\section{Heterogeneity}

Stroke survivors are heterogeneous not only with respect to aetiology and infarct or haemorrhage location, but also in terms of pre-existing clinical conditions. Brain lesions lead to a variety of syndromes (right hemisphere: recognition of distance, size, speed, and position, and self-assessment; left hemisphere: language production and understanding (aphasia)), and common comorbidities may include hypertension, hyperlipidaemia, diabetes mellitus, ECG abnormality, osteoarthritis, visual impairment, shoulder pain, depression, neurogenic bladder, and gastritis. Thus, every stroke survivor presents a unique syndrome that complicates overall comparisons in clinical research. In addition, the heterogeneity leads to a major challenge regarding time and feasibility for the recruitment of a sample that presents acceptable homogeneity to perform valid comparisons in experimental research. 
We applied strict eligibility criteria to improve the homogeneity of the samples studied. However, we could not control the sample on more detailed aspects such as stroke type, brain lesion side, and existing comorbidities due to general limitations of resources. As this research aimed to implement a novel strategy for cardiovascular rehabilitation, we focused on safety aspects (i.e. contraindications for exercise testing) and on frequent pathologies that could have influenced our results (concurrent neurological, pulmonary disease, dementia). This rough definition of the eligibility criteria has led to successful recruitment rates and increased the generalisability of our results, but biased the outcomes of the experimental approach presented here.

\section{Ethical and clinical considerations}

After the successful clinical implementation of FC-RATE for CPET, the focus shifted to the design of an intervention trial. A major difficulty was the definition of the control intervention. Although the major rehabilitation centres in Switzerland provide RATE, the method cannot be seen as standard care after stroke. We defined RATE as the active comparator to FC-RATE due to the fact that RATE is part of the standard rehabilitation program of the Reha Rheinfelden, the rehabilitation centre where this research project was conducted. The fact that CPET was based on RATE and that we aimed to evaluate the effects of intensive cardiovascular exercise on motor function reinforced our decision.

The early phase of stroke rehabilitation includes a variety of interventions and assessment procedures, which requires large resources. Inpatient rehabilitation can be very stressful, and stroke survivors present large day-by-day variability regarding form on the day. Therefore, the early stages after stroke complicate the performance of research studies, and the short inpatient period combined with the complex course of care during acute and subacute stroke leads to major challenges regarding the implementation of intensive intervention programmes for research purposes.

\section{Spontaneous recovery}

Some degree of spontaneous motor recovery is usually seen after stroke onset due to reorganisation of surviving central nervous system elements [37]. The majority of motor recovery will reach a plateau within 6 months, with some continued recovery after this period [38]. Regarding cardiovascular fitness, preliminary evidence exists that reveals a spontaneous recovery of $\dot{\mathrm{V}} \mathrm{O}_{2}$ peak of $16.9 \%$ within the first 6 months after stroke [39], which might have affected the findings of the controlled intervention study presented in this research (CHAPTER 7). The overall improvement in $\mathrm{VO}_{2}$ peak of $18 \%$ within 4 weeks in the subacute phase might be partly based on spontaneous recovery. 
In subacute stroke research, the specific effects of an intervention are always covered by a certain amount of spontaneous recovery. Well-controlled longitudinal intervention studies can distinguish between true effects of a specific intervention and spontaneous recovery, but several aspects such as heterogeneity as described above, effects sizes of the intervention approach, obligatory routine rehabilitation procedures, and a different response to the intervention can influence the outcomes.

\section{Early rehabilitation}

The sample studied here was in the subacute rehabilitation phase (6 days- 6 months post-stroke). While the first 30 days after stroke onset have shown to be a critical time period for treatment initiation [40], there is insufficient evidence that very early admission (0-6 days post-stroke) of physical activity might lead to improved rehabilitation outcomes [41]. Recent guidelines suggest preventing deconditioning, hypostatic pneumonia, and orthostatic intolerance by the implementation of exercise that increases resting HR up to 20 beats/min (RPE $\leq 11$ (6-20 scale). Based on the results in CHAPTER 3, RATE might be a potential treatment option in the very early period; however, this needs to be established in further studies.

\section{Technology}

\section{Robotics assisted treadmill exercise}

Robotics-assisted treadmill technology facilitates task-specific exercise for severely motor impaired individuals early after stroke. The technology provides controllable parameters for assessment and guidance of physical performance based on integrated mechanical sensors. Although this system has been implemented in daily clinical routine, the overall setup of the device is extensive. Subjects included in our studies underwent extended familiarisation sessions to ensure physiological gait patterns within the exoskeleton. Especially in stroke survivors, a proper setup is very important due to the high variability in motor control, postural control, coordination, and spasticity. During clinical experimentation, we excluded 3 subjects due to issues concerning the setup of the device (uncontrollable spasticity, tibia lesion due to inadequate padding, severe groin pain caused by the BWS). More sophisticated concepts for the control of walking patterns, the mechanical human-machine interaction points, and the BWS might provide solutions for these issues in the near future. 


\section{Conventional control strategies}

We have shown that motor impaired individuals exhibit cardiovascular responses when decreasing BWS or GF; but these conventional control strategies might be not appropriate for the assessment and improvement of cardiovascular fitness. Our hypothesis that a rapid decrease in BWS might be used to implement CLT in severely motor impaired individuals for the assessment of steady-state values and oxygen uptake kinetics $(\tau)$ had to be rejected. Further research on a suitable strategy to decrease BWS to a minimum while individuals are able to maintain a physiologic gait pattern might lead to more successful approaches. To date, step responses based on BWS during CLT have shown large variability and therefore insufficient reliability in test-retest situations. Nevertheless, the concept of decreasing BWS might be an important aspect in very early rehabilitation stages when appropriate controllers are available. The concept of decreasing GF, which is based on a progressive override of the position-controlled gait pattern, has also been shown not to be appropriate for severely impaired individuals. The required task of active walking without guidance was too difficult for this population. An option would be to decrease the support on the unaffected side only, while the hemiparetic side still receives full or customised guidance. Whether this concept can provoke higher cardiovascular responses needs to be tested in further studies.

\section{Feedback control mechanism}

The feedback-control mechanism was based on the rigid position-controlled approach of the Lokomat system. After subtracting the passive mechanical work rate that was needed to let the subject passive walking within the predefined trajectory, any additional force was counted as positive. As a result, interferences and even forces against the predefined gait pattern were counted as work rate. This limitation of the feedback-control mechanism might have led to a miscalculation of true work rate values. Even so, the mechanism required high coordinative skills to successfully reach higher target work rate values. Some of the subjects needed extended familiarisation not to understand the mechanism, but to translate it into a motor task. Indeed, the feedback-control approach is based on the physiological framework that additional forward forces are needed to walk faster; however, when reaching higher target work values, the amount of muscular force exceeded natural activation patterns leading to an substantial higher force rate production during the gait cycle compared to normal walking. This is an important limitation regarding the control concept of this approach.

Although the guidance of work rate was successful, the approach presented required work rate values of both legs and does not consider the severe hemiplegia of the included subjects. Subjects generally tend to exercise using the unaffected side more dominantly, which led to deviations 
from the predefined physiological gait pattern and might have facilitated unwanted compensation patterns.

\section{Assessment of exercise capacity}

\section{FC-RATE-based CPET}

The feedback-control mechanism was used to implement target work rate profiles for CPET in severely motor impaired stroke survivors. Exercise testing physiology aims to recruit large muscle groups to demand a substantial cardiovascular response (constant load testing (CLT)), and to progressively increase the target work rate until subjects reach their maximal exercise capacity or certain termination criteria (incremental exercise testing (IET)). The approach presented her was able to recruit large parts of the total body muscle mass by involving the major lower extremity muscles and some upper extremity muscles through the handrail for extra purchase. The variance in the severity of the hemiparesis severity led to different strategies to follow the target work rate (i.e. produce mechanical work rate). The different degrees of hemiparesis severity of the extremities might have led to varying muscle recruitment strategies within the feedback-control approach. This might have strongly affected the outcome variables, in particular during lower target work rates (i.e. CLT), which has shown insufficient test-retest reliability and repeatability.

Another important aspect to consider is the different strategy to produce the work rate, which could have influenced the cardiopulmonary response parameters. While conventional CPET approaches remain in smooth dynamic movement patterns by increasing the resistance during leg cycle ergometry or the walking speed and the inclination in conventional treadmill exercise, the approach presented here required more abrupt peak forces to successfully follow the target. More isolated muscle groups of the knee extensors and hip flexors might have reached fatigue in earlier stages compared to conventional CPET approaches. Whether a smoother work rate production within RATE leads to higher peak cardiopulmonary responses compared to conventional approaches need to be tested in future experimental research.

\section{Maximal exercise capacity}

CPET is a standardised procedure for the assessment of exercise capacity. Although our results demonstrated feasibility and reliability of using FC-RATE for CPET and promising results regarding assessment of peak exercise capacity in severely motor impaired individuals, the issue remains of whether the concept can meet traditional criteria for true maximal exercise capacity. The most accepted criteria for maximal aerobic capacity include plateau in $\dot{\mathrm{V}} \mathrm{O}_{2}$, respiratory exchange ratio 
$(\mathrm{RER}) \geq 1.15$, and HRpeak within 10 beats per minute of the age-predicted heart rate maximum (adjusted for subjects on beta-blocker medications) [42]. The results presented confirmed the issues concerning the achievement of maximal exercise capacity reported in previous studies. While some of our subjects reached a plateau in $\dot{\mathrm{VO}}_{2}$ response and HRpeak, we could not confirm the achievement of RER values $\geq 1.15$ at peak exercise. Interestingly, FC-RATE-based CPET yielded similar peak exercise capacity compared to previous studies without reaching RER values in the expected range. Further research is needed to gain data on cardiopulmonary responses during FC-RATE-based CPET for valid interpretation of true maximal exercise capacity.

\section{Anaerobic threshold}

The GET provides important information regarding the metabolic state of the exercising muscles. Exercising above the GET produces lactate, which causes more rapid fatigue. Research in chronic heart failure has shown improvements in the GET after 20 weeks of regular cardiovascular exercise $[43,44]$. This leads to higher exercise levels in a purely aerobic sense, resulting in higher functional capacity. Our sample studied here did not show significant improvements in the GET after the 4weeks intervention. Generally, an increased training duration might be necessary to influence the metabolic capacity of the muscles as shown previously. Further results on anaerobic threshold after stroke are important for interpretations regarding the metabolic changes during exercise.

\section{CPET standards}

The presented CPET approach did not used electrocardiography (ECG), as strongly recommended by international guidelines, for practical reasons. The setup within the robotics-assisted treadmill was too extensive to additionally control for specific cardiac termination criteria. We decided to rigorously exclude all subjects with a history of cardiac events and/or a present cardiac pathology at study onset. This strict eligibility criterion has led to large exclusion rates, as a major part of stroke survivors presents with some degree of cardiac disease, which decreases the generalisability of our approach. However, this research project aimed to implement a novel strategy and evaluate clinically feasibility in a high-risk population, which justifies this procedure.

Cardiopulmonary outcome variables are captured with sensitive instruments and raw data need to pass a specific pre-processing. Thus, the concept is prone to measurement error. Despite international guidelines for CPET [27, 42] and data pre-processing [27, 45], it is often unclear how the data were handled. We tried to define the pre-processing concept that allowed valid comparisons between the chapters presented in this thesis. Comparisons to external research might be influenced due to the usage of different instruments and various data processing approaches. 


\section{Training}

\section{Intensity, frequency, and duration}

Recommended training intensity, frequency and duration are 40-70\% of HRR, Ppeak, or $\dot{\mathrm{V}}_{2}$ peak, on 3-5 days/week, for 20-60 min per session [46, 47]. While FC-RATE in our intervention study (CHAPTER 7) achieved a significant higher training intensity compared to conventional RATE, both groups received only a low amount of training frequency and duration. This might have limited our approach to distinguish between the two groups in addition to the fact that the difference in training intensity was probably not clinically relevant.

Based on therapist-feedback, the training sessions in the intervention groups often were seen as too intensive for the participants, who seemed to not have the required fitness level to sustain the extensive FC-RATE training sessions. On the other hand, conventional RATE sessions were rated as boring and not really exhausting. This is another finding that indicates the need for welladjusted strategies to elicit physical activity within this population. A first adaption might be an incremental increase in training volume and frequency to better individualise the intervention.

\section{Responders}

About 30 genetic variations affect how individuals respond to cardiovascular training [48]. While some individuals can improve their $\dot{\mathrm{V}} \mathrm{O}_{2}$ max significantly (high responders), others get only little or no improvement (low responders). Whether this is true in the severely impaired population early after stroke needs to be established in further studies. However, this fact could lead to wrong conclusions regarding the evaluation of peak exercise capacity in cardiovascular intervention studies.

\section{Additional outcomes}

The study protocol presented in CHAPTER 6 defined additional outcome measures, i.e. gait speed, walking endurance, RMS deviation of work rate $\left(\mathrm{RMSE}_{\mathrm{P}}\right)$, standing function, and quality of life [31]. These outcomes have not been reported due to the extensive focus on the CPET results and the lack of impact considering the small sample size presented here. However, analyses on these secondary variables revealed no considerable group-time interactions. For example, although both groups improved their ambulatory status over time (FAC; $\mathrm{p}=0.006$ ), no between-group differences were found for gait speed (10MWT; $\mathrm{p}=0.43$ ) nor for walking endurance (6MWT; $p=0.14)$. Unsurprisingly, the absence of between-group differences in walking endurance measured with the 6MWT confirmed the findings of the CPET parameters reported in CHAPTER 7 [32]. 
The only significant group-time interaction was found for $\Delta \dot{\mathrm{V}} \mathrm{O}_{2} / \Delta \mathrm{P}$. The slope of the $\dot{\mathrm{VO}}_{2}$-P relation reflects the efficiency of the metabolic conversion to mechanical work rate and the mechanical efficiency of the musculoskeletal system. While the experimental group maintained $\Delta \dot{\mathrm{V}} \mathrm{O}_{2} / \Delta \mathrm{P}(+1.6 \%)$, the control group showed lower efficiency after the intervention period $(+33.5 \%)$. It could be hypothesised that training with the feedback-control mechanism leads to significantly better efficiency during FC-RATE. However, consistent and accurate quantification of the $\mathrm{VO}_{2}$-P relation during treadmill exercise is complicated due to underlying variability in motor impairment, body weight support level and handrail holding strategies. Further studies will be necessary gathering data on efficiency during FC-RATE to enable valid comparisons to be made.

\section{Implications for future research}

\section{Study sample}

To evaluate the effect of cardiovascular exercise on a variety of outcome variables early after stroke, further studies need to improve the homogeneity of the samples studied. The focus should be intensified on group stratification regarding type and location of lesion, severity level (i.e. motor impairment), and comorbidities. This will need large-scale multi-centre intervention studies. While some individuals will present severe motor impairments, others will exhibit severe cognitive restrictions that makes physical activity promotion challenging.

Because RATE cannot be seen as standard care, future studies needs to define usual care as the active comparator for the control group. Advanced measurement technology (e.g. activity tracking through motion sensors) will opens new perspectives regarding intensity control during the conventional rehabilitation process, which will improve the validity of comparisons in controlled intervention studies.

Spontaneous recovery will in any case dilute between-group comparisons and affect overall recovery measures. However, well-controlled long-term studies will be able to reveal the true effects of additional cardiovascular interventions on a variety of outcome variables.

Future research needs to focus on the controlled implementation of interventions in the very early phase after stroke. Interdisciplinary approaches are necessary to safely facilitate cardiovascular stress within this sensitive period of recovery. Well-controlled research might then be able to evaluate the effects of very early cardiovascular exercise administration on a variety of outcomes of interest. 


\section{Technology}

To successfully implement robotics-assisted treadmill technology in early rehabilitation stages, future research needs to develop sophisticated algorithms to promote active participation during robotics-assisted physical activity (i.e. assist-as-needed approach). The goal will be to increasingly challenge the stroke survivor on the affected side, while taking the capacity of the unaffected extremities into account. Considering the importance of errors or mismatch between intended and performed movements to facilitate motor learning $[49,50]$, more degrees of freedom within the exoskeleton might be an important aspect to fulfil recent standards in stroke rehabilitation. A novel approach could be the implementation of controlled error amplification in which the individual is challenged to stay close to the optimal movement trajectory. Whether these disturbances during robotics-assisted walking would lead to substantial cardiovascular responses is a part of future research.

Human-machine interaction points (e.g. BWS, extremity fixation) need to be revised to provide optimal interaction between the technology and the individual. In addition, advanced strategies such as uphill walking by increasing the gradient, the modulation of speed parameters [51], and robotics-assisted stair climbing $[52,53]$ might have potential to facilitate cardiovascular stress in severely motor impaired individuals.

\section{Assessment of exercise capacity}

The implementation of advanced algorithms to guide exercise intensity will improve the assessment of true exercise capacity in severely impaired populations. Recent standards regarding the achievement of maximal exercise capacity need to be revised for acute and subacute stroke survivors. Whether lower threshold values (i.e. RER, HRpeak) or further derivatives are more appropriate to define maximal exercise capacity needs to be evaluated in future experiments.

The evaluation of changes in the anaerobic threshold (e.g. GET) after longitudinal training interventions might be of importance to document increases in functional capacity for severely impaired population.

\section{Training}

The FC-RATE concept presented here might be predestined for high intensity interval training, which has shown to be a promising method in cardiovascular rehabilitation after stroke [54]. Bursts of concentrated effort alternated with recovery periods to maximise exercise intensity might be a potential option to overcome the issues described along with exercise testing in deconditioned 
individuals. Furthermore, short exercise bouts might increase the motivation to perform such an intensive training.

Future research will also need to focus on the implementation of appropriate cardiovascular stress in the very early stages after stroke, as some preliminary evidence confirms efficacy of very early mobilisation compared to delayed interventions [41]. The substantial increase in cardiovascular exercise intensity during conventional RATE might be a potential treatment option in this case. A progressive improvement in cardiovascular stress, initially through end-effector based robotics-assisted technology in a horizontal plane, followed by RATE or robotics-assisted stair climbing might open new perspectives in the evaluation of the effects of early cardiovascular exercise administration.

\section{Implications for clinical practice}

There is robust evidence that subacute stroke survivors may benefit from cardiovascular exercise protocols to improve $\dot{\mathrm{V}}{ }_{2}$ peak and walking endurance; but standard rehabilitation procedures have been shown not to be effective enough to improve cardiovascular fitness. Therefore, subacute stroke rehabilitation strategies need to include regular cardiovascular fitness training based on available equipment. While mildly to moderately impaired stroke survivors should exercise on conventional devices (recumbent leg cycle ergometer, treadmill), or preferably participate in outdoor programmes (Nordic walking, swimming), rehabilitation centres need to provide totalbody recumbent steppers, body weight supported treadmills, robotics-assisted treadmills, or endeffector based gait trainers for the severely motor impaired population. Cardiovascular exercise intensity should be well described and controlled based on international guidelines [42, 46, 47]. CPET is not necessary in the standard clinical setting, but might be relevant for further diagnostics and training prescription. 


\section{Main original contributions}

The research presented in this dissertation has gained knowledge in the field of cardiovascular exercise early after stroke. We have shown that subacute stroke survivors benefit from cardiovascular exercise protocols to improve exercise capacity. Standard procedures for assessment and improvement of cardiovascular fitness in mildly to moderately motor impaired individuals after stroke are safe, but there is a lack of methods for the severely impaired populations. We proposed the implementation of RATE to overcome severe motor limitations and facilitate task-specific training. Initial experimental results demonstrated that conventional control strategies during RATE are not suitable for the evaluation of exercise capacity. Further clinical experimentation using FC-RATE yielded promising results for assessment and training of cardiovascular fitness early after stroke. FC-RATE-based CPET demonstrated good to excellent test-retest reliability and acceptable repeatability for the major cardiopulmonary performance parameters. The concept has shown to be able to significantly increase the exercise intensity compared to conventional RATE, and both approaches have led to a significantly improvement in the main cardiopulmonary performance parameter within a 4-weeks intervention period. For the first time, we present a clinically feasible method for assessment and training of cardiovascular fitness in severely motor impaired stroke survivors. These findings are an important step towards the implementation and exploration of cardiovascular rehabilitation early after stroke. 


\section{References}

[1] O. Stoller, E. D. de Bruin, R. H. Knols, and K. J. Hunt, "Effects of cardiovascular exercise early after stroke: systematic review and meta-analysis," BMC Neurology, vol. 12, no. 1, p. 45, 2012.

[2] F. M. Ivey, A. S. Ryan, C. E. Hafer-Macko, and R. F. Macko, "Improved cerebral vasomotor reactivity after exercise training in hemiparetic stroke survivors," Stroke, vol. 42, no. 7, pp. 1994-2000, 2011.

[3] A. R. Luft, R. F. Macko, L. W. Forrester, F. Villagra, F. Ivey, J. D. Sorkin, J. Whitall, S. McCombe-Waller, L. Katzel, A. P. Goldberg, and D. F. Hanley, "Treadmill exercise activates subcortical neural networks and improves walking after stroke: a randomized controlled trial," Stroke, vol. 39, no. 12, pp. 3341-50, 2008.

[4] M. Ploughman, J. McCarthy, M. Bosse, H. J. Sullivan, and D. Corbett, "Does treadmill exercise improve performance of cognitive or upper-extremity tasks in people with chronic stroke? a randomized cross-over trial," Archives of Physical Medicine and Rehabilitation, vol. 89, no. 11, pp. 2041-2047, 2008.

[5] M. S. El-Tamawy, F. Abd-Allah, S. M. Ahmed, M. H. Darwish, and H. A. Khalifa, "Aerobic exercises enhance cognitive functions and brain derived neurotrophic factor in ischemic stroke patients," NeuroRehabilitation, vol. 34, no. 1, pp. 209-13, 2014.

[6] P. M. Kluding, B. Y. Tseng, and S. A. Billinger, "Exercise and executive function in individuals with chronic stroke: a pilot study," J Neurol Phys Ther, vol. 35, no. 1, pp. 11-7, 2011.

[7] S. Marzolini, P. Oh, W. McIlroy, and D. Brooks, "The effects of an aerobic and resistance exercise training program on cognition following stroke," Neurorehabil Neural Repair, vol. 27, no. 5, pp. 392-402, 2013.

[8] B. M. Quaney, L. A. Boyd, J. M. McDowd, L. H. Zahner, J. H. He, M. S. Mayo, and R. F. Macko, "Aerobic exercise improves cognition and motor function poststroke," Neurorehabilitation and Neural Repair, vol. 23, no. 9, pp. 879-885, 2009.

[9] O. Stoller, E. D. de Bruin, M. Schindelholz, C. Schuster, R. A. de Bie, and K. J. Hunt, "Evaluation of exercise capacity after severe stroke using robotics-assisted treadmill exercise: A proof-of-concept study," Technology and Health Care, vol. 21, no. 2, pp. 157-166, 2013.

[10] C. Krewer, F. Müller, B. Husemann, S. Heller, J. Quintern, and E. Koenig, "The influence of different lokomat walking conditions on the energy expenditure of hemiparetic patients and healthy subjects," Gait Posture, vol. 26, no. 3, pp. 372-377, 2007.

[11] M. P. van Nunen, K. H. Gerrits, A. de Haan, and T. W. Janssen, "Exercise intensity of robot-assisted walking versus overground walking in nonambulatory stroke patients," J Rehabil Res Dev, vol. 49, no. 10, pp. 1537-46, 2012 .

[12] J. F. Israel, D. D. Campbell, J. H. Kahn, and T. G. Hornby, "Metabolic costs and muscle activity patterns during robotic- and therapist-assisted treadmill walking in individuals with incomplete spinal cord injury," Physical Therapy, vol. 86, no. 11, pp. 1466-1478, 2006.

[13] L. P. Jack, M. Purcell, D. B. Allan, and K. J. Hunt, "The metabolic cost of passive walking during roboticsassisted treadmill exercise," Technology and Health Care, vol. 19, no. 1, pp. 21-27, 2011.

[14] O. Stoller, M. Schindelholz, L. Bichsel, C. Schuster, R. A. de Bie, E. D. de Bruin, and K. J. Hunt, "Feedbackcontrolled robotics-assisted treadmill exercise to assess and influence aerobic capacity early after stroke: a proofof-concept study," Disabil Rehabil Assist Technol, vol. 9, no. 4, pp. 271-278, 2014.

[15] A. Tang, K. M. Sibley, S. G. Thomas, W. E. McIlroy, and D. Brooks, "Maximal exercise test results in subacute stroke," Archives of Physical Medicine and Rehabilitation, vol. 87, no. 8, pp. 1100-1105, 2006.

[16] J. J. Eng, A. S. Dawson, and K. S. Chu, "Submaximal exercise in persons with stroke: Test-retest reliability and concurrent validity with maximal oxygen consumption," Archives of Physical Medicine and Rehabilitation, vol. 85, no. 1, pp. 113-118, 2004. 
[17] J. K. Chen, T. W. Chen, C. H. Chen, and M. H. Huang, "Preliminary study of exercise capacity in post-acute stroke survivors," Kaohsiung Journal of Medical Sciences, vol. 26, no. 4, pp. 175-181, 2010.

[18] J. S. Yates, S. Studenski, S. Gollub, R. Whitman, S. Perera, S. M. Lai, and P. W. Duncan, "Bicycle ergometry in subacute-stroke survivors: Feasibility, safety, and exercise performance," Journal of Aging and Physical Activity, vol. 12, no. 1, pp. 64-74, 2004.

[19] P. J. Manns, C. R. Tomczak, A. Jelani, and R. G. Haennel, “Oxygen uptake kinetics: associations with ambulatory activity and physical functional performance in stroke survivors," J Rehabil Med, vol. 42, no. 3, pp. 259-264, 2010 .

[20] O. Stoller, E. D. de Bruin, M. Schindelholz, C. Schuster-Amft, R. A. de Bie, and K. J. Hunt, "Cardiopulmonary exercise testing early after stroke using feedback-controlled robotics-assisted treadmill exercise: test-retest reliability and repeatability," Journal of Neuroengineering and Rehabilitation, vol. 11, p. 145, 2014.

[21] M. J. MacKay-Lyons and L. Makrides, "Exercise capacity early after stroke," Archives of Physical Medicine and Rehabilitation, vol. 83, no. 12, pp. 1697-1702, 2002.

[22] J. O. Kelly, S. L. Kilbreath, G. M. Davis, B. Zeman, and J. Raymond, "Cardiorespiratory fitness and walking ability in subacute stroke patients," Archives of Physical Medicine Rehabilitation, vol. 84, no. 12, pp. 1780$1785,2003$.

[23] D. C. Hill, K. A. Ethans, D. A. MacLeod, E. R. Harrison, and J. E. Matheson, "Exercise stress testing in subacute stroke patients using a combined upper- and lower-limb ergometer," Archives of Physical Medicine and Rehabilitation, vol. 86, no. 9, pp. 1860-1866, 2005.

[24] C. L. Dobrovolny, F. M. Ivey, M. A. Rogers, J. D. Sorkin, and R. F. Macko, "Reliability of treadmill exercise testing in older patients with chronic hemiparetic stroke," Archives of Physical Medicine Rehabilitation, vol. 84, no. 9, pp. 1308-12, 2003.

[25] O. C. Lennon, R. S. Denis, N. Grace, and C. Blake, "Feasibility, criterion validity and retest reliability of exercise testing using the astrand-rhyming test protocol with an adaptive ergometer in stroke patients," Disabil Rehabil, vol. 34 , no. 14 , pp. 1149-56, 2012.

[26] C. Olivier, J. Dore, S. Blanchet, D. Brooks, C. L. Richards, G. Martel, N. M. Robitaille, and D. B. Maltais, "Maximal cardiorespiratory fitness testing in individuals with chronic stroke with cognitive impairment: practice test effects and test-retest reliability," Arch Phys Med Rehabil, vol. 94, no. 11, pp. 2277-82, 2013.

[27] "Ats/accp statement on cardiopulmonary exercise testing," American Journal of Respiratory and Critical Care Medicine, vol. 167, pp. 211-277, 2003.

[28] C. S. Garrard and C. Emmons, "The reproducibility of the respiratory responses to maximum exercise," Respiration, vol. 49, no. 2, pp. 94-100, 1986.

[29] J. E. Nordrehaug, R. Danielsen, L. Stangeland, G. A. Rosland, and H. Vik-Mo, "Respiratory gas exchange during treadmill exercise testing: reproducibility and comparison of different exercise protocols," Scand J Clin Lab Invest, vol. 51, no. 7, pp. 655-8, 1991.

[30] A. Barron, N. Dhutia, J. Mayet, A. D. Hughes, D. P. Francis, and R. Wensel, "Test-retest repeatability of cardiopulmonary exercise test variables in patients with cardiac or respiratory disease," Eur J Prev Cardiol, vol. 21, no. 4, pp. 445-53, 2014.

[31] O. Stoller, E. D. de Bruin, C. Schuster-Amft, M. Schindelholz, R. A. de Bie, and K. J. Hunt, "Cardiovascular rehabilitation soon after stroke using feedback-controlled robotics-assisted treadmill exercise: study protocol of a randomised controlled pilot trial," Trials, vol. 14, no. 1, p. 304, 2013.

[32] O. Stoller, E. D. de Bruin, M. Schindelholz, C. Schuster-Amft, R. A. de Bie, and K. J. Hunt, "Efficacy of feedback-controlled robotics-assissted treadmill exercise to improve cardiovascular fitness early after stroke: a randomized controlled pilot trial," Journal of Neurologic Physical Therapy, submitted. 
[33] C. E. Garber, B. Blissmer, M. R. Deschenes, B. A. Franklin, M. J. Lamonte, I. M. Lee, D. C. Nieman, D. P. Swain, and M. American College of Sports, "American college of sports medicine position stand. quantity and quality of exercise for developing and maintaining cardiorespiratory, musculoskeletal, and neuromotor fitness in apparently healthy adults: guidance for prescribing exercise," Med Sci Sports Exerc, vol. 43, no. 7, pp. 1334-59, 2011.

[34] W. H. Chang, M. S. Kim, J. P. Huh, P. K. W. Lee, and Y. H. Kim, "Effects of robot-assisted gait training on cardiopulmonary fitness in subacute stroke patients: A randomized controlled study," Neurorehabilitation and Neural Repair, vol. 26, no. 4, pp. 318-324, 2012.

[35] R. Hambrecht, C. Walther, S. Mobius-Winkler, S. Gielen, A. Linke, K. Conradi, S. Erbs, R. Kluge, K. Kendziorra, O. Sabri, P. Sick, and G. Schuler, "Percutaneous coronary angioplasty compared with exercise training in patients with stable coronary artery disease: a randomized trial," Circulation, vol. 109, no. 11, pp. 1371-8, 2004.

[36] J. Myers, M. Prakash, V. Froelicher, D. Do, S. Partington, and J. E. Atwood, "Exercise capacity and mortality among men referred for exercise testing," New England Journal of Medicine, vol. 346, no. 11, pp. 793-801, 2002.

[37] S. C. Cramer, "Repairing the human brain after stroke: I. mechanisms of spontaneous recovery," Ann Neurol, vol. 63, no. 3, pp. 272-87, 2008.

[38] J. B. Green, “Brain reorganization after stroke,” Top Stroke Rehabil, vol. 10, no. 3, pp. 1-20, 2003.

[39] M. J. MacKay-Lyons and L. Makrides, "Longitudinal changes in exercise capacity after stroke," Archives of Physical Medicine and Rehabilitation, vol. 85, no. 10, pp. 1608-1612, 2004.

[40] J. W. Krakauer, S. T. Carmichael, D. Corbett, and G. F. Wittenberg, "Getting neurorehabilitation right: what can be learned from animal models?," Neurorehabil Neural Repair, vol. 26, no. 8, pp. 923-31, 2012.

[41] J. Bernhardt, M. N. T. Thuy, J. M. Collier, and L. A. Legg, "Very early versus delayed mobilization after stroke," Stroke, vol. 40, no. 7, pp. e489-e490, 2009.

[42] W. R. Thompson, N. F. Gordon, and L. S. Pescatello, American College of Sports Medicine. Guidelines for Exercise Testing and Prescription. Philadelphia: Lippincott Williams Wilkins, eight ed., 2010.

[43] R. Hambrecht, J. Niebauer, E. Fiehn, B. Kalberer, B. Offner, K. Hauer, U. Riede, G. Schlierf, W. Kubler, and G. Schuler, "Physical training in patients with stable chronic heart failure: effects on cardiorespiratory fitness and ultrastructural abnormalities of leg muscles," J Am Coll Cardiol, vol. 25, no. 6, pp. 1239-49, 1995.

[44] M. J. Sullivan, M. B. Higginbotham, and F. R. Cobb, "Exercise training in patients with chronic heart failure delays ventilatory anaerobic threshold and improves submaximal exercise performance," Circulation, vol. 79, no. 2, pp. 324-9, 1989.

[45] R. A. Robergs, D. Dwyer, and T. Astorino, "Recommendations for improved data processing from expired gas analysis indirect calorimetry," Sports Medicine, vol. 40, no. 2, pp. 95-111, 2010.

[46] N. F. Gordon, M. Gulanick, F. Costa, G. Fletcher, B. A. Franklin, E. J. Roth, and T. Shephard, "Physical activity and exercise recommendations for stroke survivors - an american heart association scientific statement from the council on clinical cardiology, subcommittee on exercise, cardiac rehabilitation, and prevention; the council on cardiovascular nursing; the council on nutrition, physical activity, and metabolism; and the stroke council," Stroke, vol. 35, no. 5, pp. 1230-1240, 2004.

[47] S. A. Billinger, R. Arena, J. Bernhardt, J. J. Eng, B. A. Franklin, C. M. Johnson, M. MacKay-Lyons, R. F. Macko, G. E. Mead, E. J. Roth, M. Shaughnessy, A. Tang, C. American Heart Association Stroke, C. Council on, N. Stroke, L. Council on, H. Cardiometabolic, E. Council on, Prevention, and C. Council on Clinical, "Physical activity and exercise recommendations for stroke survivors: a statement for healthcare professionals from the american heart association/american stroke association," Stroke, vol. 45, no. 8, pp. 2532-53, 2014. 
[48] C. Bouchard, P. An, T. Rice, J. S. Skinner, J. H. Wilmore, J. Gagnon, L. Perusse, A. S. Leon, and D. C. Rao, "Familial aggregation of vo(2max) response to exercise training: results from the heritage family study," J Appl Physiol (1985), vol. 87, no. 3, pp. 1003-8, 1999.

[49] J. Diedrichsen, O. White, D. Newman, and N. Lally, "Use-dependent and error-based learning of motor behaviors," J Neurosci, vol. 30, no. 15, pp. 5159-66, 2010.

[50] D. M. Wolpert and R. C. Miall, "Forward models for physiological motor control," Neural Netw, vol. 9, no. 8, pp. 1265-1279, 1996

[51] L. P. Jamieson, K. J. Hunt, and D. B. Allan, "A treadmill control protocol combining nonlinear, equally smooth increases in speed and gradient: Exercise testing for subjects with gait and exercise limitations," Medical Engineering and Physics, vol. 30, no. 6, pp. 747-754, 2008.

[52] S. Hesse, A. Waldner, and C. Tomelleri, "Innovative gait robot for the repetitive practice of floor walking and stair climbing up and down in stroke patients," J Neuroeng Rehabil, vol. 7, p. 30, 2010.

[53] O. Stoller, M. Schindelholz, L. Bichsel, and K. J. Hunt, "Cardiopulmonary responses to robotic end-effectorbased walking and stair climbing," Medical Engineering and Physics, vol. 36, no. 4, pp. 425-31, 2014.

[54] P. Boyne, K. Dunning, D. Carl, M. Gerson, J. Khoury, and B. Kissela, "High-intensity interval training in stroke rehabilitation," Top Stroke Rehabil, vol. 20, no. 4, pp. 317-30, 2013. 



\section{Summary / Zusammenfassung}




\section{Summary}

This dissertation addresses the implementation of cardiovascular exercise early after severe stroke. The aims were: (1) to review the knowledge about the effects of cardiovascular exercise early after stroke, (2) to implement a robotic-based strategy to accomplish recent standards for exercise testing and prescription for severely motor impaired stroke survivors, and (3) to systematically evaluate the concept within a clinical setting.

CHAPTER 1 provides an overview of the current knowledge on cardiovascular exercise and health, the state-of-the-art in the assessment of exercise capacity and its interpretation, the recent standards in stroke rehabilitation with a focus on cardiovascular health, and the role of rehabilitation robotics.

The human species is designed to perform prolonged physical exercise. Unsurprisingly, cardiovascular exercise might be responsible for several health-conserving factors. Evidence clearly indicates that individuals who are active tend to live longer and have lower risk for a variety of cardiovascular related diseases.

Each year about 16 million people worldwide experience a stroke. The primary rehabilitation goals are to reduce brain injury and promote maximal recovery. Once a stroke survivor is medically stable, the focus shifts to the prevention of secondary health complications, minimising impairments, and achieving functional goals that promote independence in activities of daily living. The majority of stroke survivors present with cardiovascular related health issues. This limits performance of physical activity and may contribute to a heightened risk for recurrent stroke and cardiovascular disease. Research has shown that the exercise capacity of stroke survivors is 25 $60 \%$ lower than in age-matched, healthy individuals. As a consequence, the early and persistent decline in exercise capacity can delay or inhibit participation in exercise programmes, complicate the rehabilitation process and long-term post-stroke course of care, and limit the ability of the individual to perform functional activities independently.

While studies in chronic stroke ( $>6$ month after the event) have shown efficacy for cardiovascular exercise to improve exercise capacity, the early rehabilitation period presents unique challenges due to severe motor impairments. Here, rehabilitation robotics might have large potential to implement appropriate cardiovascular stress to improve exercise capacity.

The main hypothesis driving this thesis is that the introduction of cardiovascular exercise early after stroke will positively influence cardiovascular health and exercise capacity, which might facilitate overall recovery. A potential methodology for assessment of cardiovascular fitness and exercise prescription for stroke survivors with severe motor limitations could enable further re- 
search regarding the effects of early cardiovascular exercise administration on a variety of further key outcomes (i.e. neuroplasticity, quality of life).

CHAPTER 2 comprises a systematic review and meta-analysis regarding the current knowledge about the effects of cardiovascular exercise interventions early after stroke. The results indicated robust evidence that subacute stroke survivors benefit from cardiovascular exercise protocols to improve peak oxygen uptake and walking endurance. Further measures on motor recovery, functional ability and quality of life did not consistently improve, and large heterogeneity in the applied outcome measurements was found that restricted further synthesises of the results. Previously reported outcome variables in chronic stroke such as cerebral blood flow, neuroplasticity, and cognition, were not addressed in the included studies. As a result, the efficacy of cardiovascular exercise early after stroke on motor recovery, functional ability, quality of life, cerebral blood flow, neuroplasticity and cognition remains unknown.

Cardiovascular exercise training and the assessment of cardiovascular fitness using standardised cardiopulmonary exercise testing have demonstrated clinical safety. None of the reviewed studies reported major adverse events. To date, research included mildly to moderately impaired stroke survivors only. These individuals are able to perform on established methods such as semirecumbent leg cycle ergometers, conventional leg cycle ergometers, or treadmills. The review identified a lack of evidence for the severely motor impaired stroke population due to unique challenges in motor control. Furthermore, new concepts for assessment and improvement of exercise capacity in severely disabled individuals are sought allowing to explore potential effects of early cardiovascular exercise administration.

CHAPTER 3 describes a potential approach to overcome severe motor limitations while facilitating cardiovascular stress using robotics-assisted treadmill exercise (RATE). Standardised cardiopulmonary exercise testing protocols (constant load testing, incremental exercise testing) were implemented to assess and guide exercise intensity during RATE. A first proof-of-concept study using a body weight support-based control strategy for constant load testing demonstrated the feasibility of provoking substantial cardiovascular stress; however, the step increases were not distinct enough to accurately calculate cardiopulmonary performance parameters. A further control strategy based on guidance force reduction was not appropriate to elicit peak cardiopulmonary responses for the assessment of exercise capacity during incremental exercise testing. These findings are in line with previous studies that have shown that conventional RATE is not passive, and the control of body weight support and guidance force has only a minor influence on cardiopulmonary performance parameters. The major issue of both strategies was to provide an appropriate chal- 
lenge level while simultaneously supporting the severe motor impairments of the individuals. As a result, recommended intensity for assessment and improvement of exercise capacity in severely impaired individuals could not be achieved using these conventional control strategies.

CHAPTER 4 presents a novel assessment protocol that controls active participation during RATE by incorporating a biofeedback mechanism. This method allows individuals to maximise their voluntary effort and associated cardiovascular stress. The concept of feedback-controlled roboticsassisted treadmill exercise (FC-RATE) demonstrated feasibility for guidance of exercise intensity and assessment of exercise capacity in severely motor impaired individuals. Results yielded reasonable cardiopulmonary performance parameters compared to previous studies on conventional cardiopulmonary exercise testing. Individuals demonstrated high accuracy of work rate tracking, which means that subjects were able to follow the target work rate during walking on the roboticsassisted treadmill. As a result, the FC-RATE concept has been taken for further consideration as a potential method for assessment of exercise capacity and guidance of exercise intensity in severely motor impaired individuals early after stroke.

CHAPTER 5 investigates the reliability and the repeatability of FC-RATE-based cardiopulmonary exercise testing early. Peak cardiopulmonary performance parameters during incremental exercise testing were slightly higher compared to previous studies in mildly to moderately impaired stroke survivors using mostly leg-cycle ergometry. This finding might be based on the introduction of treadmill exercise that has previously confirmed higher peak values compared to leg cycle ergometry protocols. FC-RATE-based cardiopulmonary exercise testing confirmed good to excellent relative reliability and acceptable absolute reliability for the major peak cardiopulmonary performance parameters. The findings on reliability are in line with previous studies using conventional approaches. Although studies in healthy subjects and individuals with cardiac or respiratory disease have shown excellent repeatability, the present study yielded considerably higher variability for the major cardiopulmonary parameters. Cardiopulmonary exercise testing revealed high mean differences and insufficient test-retest reliability for all variables studied. Sudden onset of changes in body weight support, walking patterns, and target work rate values seem to have a strong impact on individual performance levels and provoke high variability in the measured parameters, which restricts acquisition of valid measures.

CHAPTER 6 defines a study protocol and presents the theoretical rationale for a randomised controlled pilot trial comparing FC-RATE with conventional RATE regarding cardiovascular rehabilitation. Subjects are recruited early after their first stroke ( $<20$ weeks) and then randomly allocated to an inpatient cardiovascular exercise programme that uses FC-RATE (experimental) 
or to conventional RATE (control). The training intensity of the experimental group is defined at $40-70 \%$ of heart rate reserve, whereas the control group receives low intensity exercise $(<30 \%$ heart rate reserve). Outcome variables focus on exercise capacity and ambulatory function, and are conducted at baseline, after each 4-weeks intervention period, and before clinical discharge.

CHAPTER 7 presents the findings of the randomised controlled pilot trial described in CHAPTER 6. Results indicated that cardiovascular exercise intensity can be significantly increased using FCRATE, but the method was not superior to conventional RATE to improve cardiovascular fitness within a 4-weeks intervention. It seems plausible that the difference in training intensity, duration, and frequency between the two groups was not high enough to express the superiority of FC-RATE over conventional RATE. The control group might have profited from regular moderate cardiovascular exercise, which has shown to be effective in deconditioned persons. We cannot distinguish the differences between spontaneous recovery and the real treatment effect due to the lack of a control group, which received usual care only. However, the spontaneous improvement in exercise capacity reported after 20 weeks post-stroke was achieved within 4-weeks of regular cardiovascular exercise in both groups. This is a promising finding regarding cardiovascular rehabilitation early after stroke.

CHAPTER 8 discusses the results, presents limitations and methodological considerations, provides recommendations for clinical practice and future research, and completes with the main original contributions of the thesis. The implementation of cardiovascular exercise early after stroke presents unique challenges. FC-RATE presents a methodology for assessment of exercise capacity and guidance of cardiovascular rehabilitation for severely motor impaired individuals. The method is able to recruit large parts of total body muscle mass, which enabled a reliable evaluation of cardiovascular fitness and a significant higher training intensity compared to conventional RATE. However, the heterogeneity of the study sample, issues based on the early rehabilitation phase after stroke, and the spontaneous recovery, affects the interpretation and the generalisation of the results. Although FC-RATE has shown to be successful in the guidance of work rate profiles, the approach was based on a position-controlled approach and did not consider the severe hemiplegia of the included subjects. Future research needs to focus on advanced robotic systems and on sophisticated algorithms to promote active participation during robotics-assisted exercise. This is expected to lead to an approximation on true maximal exercise capacity during cardiopulmonary exercise testing and to better control of cardiovascular exercise intensity.

In conclusion, we have shown that subacute stroke survivors benefit from cardiovascular exercise protocols to improve exercise capacity and proposed the implementation of RATE to overcome 
severe motor limitations and facilitate task-specific training. FC-RATE-based cardiopulmonary exercise testing demonstrated clinical feasibility, good to excellent test-retest reliability, and acceptable repeatability for the major cardiopulmonary performance parameters. The method has shown to be able to significantly increase the exercise intensity compared to conventional RATE, and both approaches have led to a significant improvement in the main cardiopulmonary performance parameter within a 4-weeks intervention period. For the first time, we present a method for assessment and training of cardiovascular fitness in severely motor impaired stroke survivors. These findings are an important step towards the implementation and exploration of cardiovascular rehabilitation early after stroke. 


\section{Zusammenfassung}

Diese Dissertation befasst sich mit der Implementierung von kardiovaskulärem Training nach einem schweren Schlaganfall. Die Ziele waren: (1) die Aufarbeitung des Wissens bezüglich den Effekten von kardiovaskulärem Training in der Frühphase nach einem Schlaganfall, (2) die Implementierung einer roboterbasierten Strategie, um aktuelle Standards in der Leistungstestung und der Trainingssteuerung bei motorisch schwer betroffenen Schlaganfallpatienten zu erreichen und (3) die systematische Evaluation des Konzepts in einem klinischen Umfeld.

KAPITEL 1 liefert einen Überblick über den aktuellen Wissensstand im Bereich kardiovaskulärer Fitness und Gesundheit, über den aktuellen Stand betreffend der Evaluation der Leistungsfähigkeit und deren Interpretation, über den aktuellen Standard in der Rehabilitation nach einem Schlaganfall mit dem Fokus auf kardiovaskuläre Gesundheit und über die Rolle der Robotik in der Rehabilitation.

Der Mensch ist konzipiert für langandauernde körperliche Aktivität. Es ist somit nicht überraschend, dass kardiovaskuläres Training für mehrere gesundheitserhaltende Faktoren verantwortlich sein könnte. Es ist erwiesen, dass aktive Menschen länger leben und ein geringeres Risiko für eine Vielzahl von Herz-Kreislauf-Erkrankungen aufweisen.

Jedes Jahr erleiden weltweit etwa 16 Millionen Menschen einen Schlaganfall. Das primäre Rehabilitationsziel ist eine Verringerung der Hirnverletzung, um eine maximale Erholung zu begünstigen. Sobald ein Schlaganfallpatient medizinisch stabil ist, steht die Prävention von sekundären Komplikationen, die Minimierung von Einschränkungen, das Erreichen von funktionellen Zielen und die Unabhängigkeit in Alltagsaktivitäten im Vordergrund. Die Mehrheit der Schlaganfallpatienten weist Herz-Kreislauf-Erkrankungen auf, was die körperliche Leistungsfähigkeit limitiert und das Risiko für einen Rückfall und weitere kardiovaskuläre Erkrankungen erhöht. Die Forschung zeigt, dass die körperliche Leistungsfähigkeit von Schlaganfallpatienten $25-60 \%$ niedriger ist als bei gleichaltrigen gesunden Personen. Diese frühe und anhaltende Verminderung der Leistungskapazität kann die Teilnahme an Trainingsprogrammen verzögern oder verhindern, den Rehabilitationsprozess und die weitere Betreuung erschweren und die Fähigkeit, funktionelle Alltagsaktivitäten auszuführen, limitieren.

Während Studien in der chronischen Phase nach einem Schlaganfall ( $>6$ Monate nach dem Ereignis) die Wirksamkeit von kardiovaskulärem Training zur Steigerung der Leistungsfähigkeit gezeigt haben, steht die Frührehabilitation nach einem Schlaganfall aufgrund der schweren motorischen Beeinträchtigungen der Patienten vor besonderen Herausforderungen. Die Rehabilitati- 
onsrobotik hat hier grosses Potenzial, angepasste kardiovaskuläre Belastungen einzuführen um die körperliche Leistungsfähigkeit in der Frühphase nach einem Schlaganfall zu verbessern.

Die folgende Arbeit basiert auf der Hypothese, dass die Einführung von kardiovaskulärem Training die kardiovaskuläre Gesundheit und die körperliche Leistungsfähigkeit steigern und somit die allgemeine Erholung positiv beeinflussen könnte. Eine geeignete Methode zur Beurteilung der kardiovaskulären Fitness und zur Verabreichung von Trainingsprogrammen für Schlaganfallpatienten mit schweren motorischen Einschränkungen könnte die weitere Forschung in Bezug auf die Effekte von frühem kardiovaskulärem Training auf wichtige Messparameter (z.B. Neuroplastizität, Lebensqualität) entscheidend vorantreiben.

KAPITEL 2 umfasst eine systematische Literaturübersicht und eine Meta-Analyse der Effekte von kardiovaskulärem Training nach einem Schlaganfall. Die Ergebnisse zeigen mit robuster Beweiskraft, dass subakute Schlaganfallpatienten von Ausdauertraining profitieren und dadurch die Leistungskapazität und die Gangausdauer steigern können. Weitere Messvariablen, wie motorische Erholung, Funktionsfähigkeit und Lebensqualität, haben sich nicht konsistent verbessert. Des Weiteren hat eine hohe Heterogenität der Messmethoden weitere Synthesen der Ergebnisse verhindert. Zuvor verwendete Messvariablen bei chronischen Schlaganfallpatienten wie zerebraler Blutfluss, Neuroplastizität und Kognition wurden in den eingeschlossenen Studien nicht berücksichtigt. Somit bleibt die Wirksamkeit von Ausdauertraining in der Frühphase nach einem Schlaganfall auf die motorische Erholung, die Funktionsfähigkeit, die Lebensqualität, den zerebralen Blutfluss, die Neuroplastizität und die Kognition unbekannt.

Die klinische Sicherheit des kardiovaskulären Trainings und der kardiopulmonalen Leistungstestung konnte für die Frühphase nach einem Schlaganfall gezeigt werden. Keine der eingeschlossenen Studien musste schwerwiegende Ereignisse berichten. Bisher durchgeführte Studien haben lediglich leicht bis mässig beeinträchtigte Schlaganfallpatienten einschlossen, welche motorisch in der Lage waren, auf etablierten Geräten, wie z.B. einem Liege-Fahrrad-Ergometer oder einem konventionellen Fahrradergometer, zu treten oder auf einem Laufband zu gehen. Es mangelt an Evidenz im Bereich der schwer betroffenen Schlaganfallpatienten aufgrund von Limitierungen der motorischen Kontrolle. Neue Konzepte zur Evaluation und Verbesserung der körperlichen Leistungsfähigkeit bei motorisch schwerbehinderten Personen sind nötig, um mögliche Auswirkungen einer frühen kardiovaskulären Trainingsaufnahme zu evaluieren.

KAPITEL 3 beschreibt eine potentielle Methode, die mit Hilfe eines robotergestützten Laufbands schwere motorische Einschränkungen überwindet um gleichzeitig das Herzkreislaufsystem zu belasten. Es wurden standardisierte kardiopulmonale Leistungstestprotokolle implementiert (kon- 
stante Stufentests, inkrementelle Rampentests), um die Trainingsintensität zu evaluieren und zu steuern. Eine erste Machbarkeitsstudie, welche die Reduktion der Körpergewichtsentlastung zur Implementierung von konstanten Stufentests nutzte, zeigte eine erhebliche Steigerung der Herzkreislaufbelastung. Die kardiovaskuläre Antwort auf die Erhöhungen der Stufen war jedoch nicht ausgeprägt genug, um kardiopulmonale Messparameter berechnen zu können. Eine weitere Steuerungsstrategie, welche auf der Reduktion der Führungskraft der robotergesteuerten Orthese basierte, war ebenfalls nicht geeignet, um submaximale kardiopulmonale Messparameter zur Evaluation der Leistungsfähigkeit während inkrementeller Rampentests zu provozieren. Diese Ergebnisse stehen im Einklang mit früheren Studien, welche bestätigt haben, dass herkömmliches robotergestütztes Laufbandtraining zwar nicht passiv ist, die Kontrolle der Körpergewichtsentlastung und der Führungskraft jedoch nur einen geringen Einfluss auf die kardiovaskulären Leistungsparameter haben. Das Hauptproblem beider Strategien war die Schwierigkeit, gleichzeitig eine geeignete motorische Herausforderung und eine optimale Unterstützung der schweren motorischen Beeinträchtigungen zu bieten. Die Resultate haben gezeigt, dass die empfohlene Intensität für die Evaluation und die Verbesserung der körperlichen Leistungsfähigkeit bei motorisch stark eingeschränkten Personen mit diesen herkömmlichen Regelungsstrategien nicht erreicht werden konnte.

KAPITEL 4 stellt ein neuartiges Evaluationsprotokoll vor, welches die aktive Teilnahme anhand eines Biofeedback-Mechanismus steuert. Diese Methode ermöglicht es, den aktiven Einsatz zu maximieren und somit die Herzkreislaufbelastung zu steigern. Feedback-kontrolliertes, robotergestütztes Laufbandtraining hat die Machbarkeit in Bezug auf die Steuerung der Trainingsintensität und der Evaluation der Leistungskapazität in einer weiteren Machbarkeitsstudie mit motorisch schwer betroffenen Schlaganfallpatienten gezeigt. Die Ergebnisse waren vergleichbar mit den Resultaten vorangegangener Studien, welche konventionelle Methoden zur kardiopulmonalen Leistungstestung in der Frühphase nach einem Schlaganfall angewendet haben. Die Probanden zeigten eine hohe Genauigkeit im Erreichen der Leistungsvorgabe. Feedback-kontrolliertes, robotergestütztes Laufbandtraining wurde somit als potentielles Verfahren zur Beurteilung der körperlichen Leistungsfähigkeit und Steuerung der Trainingsintensität bei schwer betroffenen Patienten akzeptiert und sollte weiter überprüft werden.

KAPITEL 5 untersucht die Reliabilität und die Wiederholbarkeit von kardiopulmonaler Leistungstestung mittels eines feedback-kontrollierten, robotergestützten Laufbandes in der Frühphase nach einem Schlaganfall. Kardiopulmonale Leistungsparameter während inkrementeller Rampentests waren etwas höher im Vergleich zu früheren Studien mit leicht bis mässig motorisch limitierten Patienten, wobei die Tests vorwiegend auf Fahrrad-Ergometern durchgeführt wurden. Diese 
Resultate könnten auf der Einführung des Laufbandtrainings basieren, welches höhere Spitzenwerte im Vergleich zur Fahrrad-Ergometrie gezeigt hat. Die neue Methode erreichte eine gute bis ausgezeichnete relative Reliabilität und eine akzeptable absolute Reliabilität in den wesentlichen kardiopulmonalen Leistungsparametern. Diese Resultate bestätigen die Ergebnisse von vorhergehenden Studien, welche konventionelle Methoden zur Leistungstestung verwendeten. Obwohl Studien mit gesunden Probanden und Patienten mit Herzkreislauf- oder Atemwegserkrankungen eine sehr gute Wiederholbarkeit zeigten, fand die vorliegende Studie eine deutlich höhere Variabilität in den wichtigsten Leistungsparametern. Die konstanten Stufentests zeigten hohe Differenzen der Mittelwerte und eine unzureichende Reliabilität. Plötzliche Veränderungen der Körpergewichtsentlastung, des Gangmusters und der Leistungsvorgabe scheinen die individuelle Leistungsfähigkeit stark zu beeinflussen und eine hohe Variabilität der Messparameter zu provozieren, was zuverlässige Messungen verhindert.

KAPITEL 6 definiert ein Studienprotokoll einer randomisierten, kontrollierten Pilotstudie mit dem Ziel, feedback-kontrolliertes, robotergestütztes Laufbandtraining mit konventionellem, robotergestütztem Laufbandtraining hinsichtlich der Wirksamkeit zu prüfen. Die Patienten werden frühzeitig nach einem Schlaganfall ( $<20$ Wochen) rekrutiert und zufällig einem stationären, feedback-kontrollierten, robotergestützten Laufbandtraining (Experimental-Gruppe) oder einem herkömmlichen, robotergestützten Laufbandtraining (Kontroll-Gruppe) zugeordnet. Die Trainingsintensität der Experimentalgruppe wird bei 40-70\% der Herzfrequenzreserve festgesetzt, während die Kontrollgruppe herkömmliches, robotergestütztes Laufbandtraining mit einer niedrigen Intensität erhält ( $<30 \%$ Herzfrequenzreserve). Die Messvariablen konzentrieren sich auf die kardiovaskuläre Kapazität und die Gehfähigkeit und werden zu Beginn der Studie, nach jeder 4-wöchigen Interventionsperiode und vor der klinischen Entlassung aufgenommen.

KAPITEL 7 präsentiert die Ergebnisse der in KAPITEL 6 beschrieben randomisierten, kontrollierten Pilotstudie. Die Resultate zeigten, dass feedback-kontrolliertes, robotergestütztes Laufbandtraining die Trainingsintensität bei motorisch schwer beeinträchtigten Schlaganfallpatienten signifikant erhöhen kann. Die Methode war jedoch betreffend der Steigerung der kardiovaskulären Fitness nach einer 4-wöchigen Intervention nicht besser als herkömmliches, robotergestütztes Laufbandtraining. Der Unterschied zwischen den beiden Gruppen bezüglich Trainingsintensität, -dauer und -frequenz war vermutlich nicht hoch genug, um die Überlegenheit von feedback-kontrolliertem, robotergestütztem Laufbandtraining gegenüber herkömmlichem, robotergestütztem Laufbandtraining zu beweisen. Die Kontrollgruppe hat möglicherweise von moderatem Herzkreislauftraining profitiert, welches vor allem bei dekonditionierten Individuen zu einer Steigerung der kardiovas- 
kulären Fitness führt. Es konnte aufgrund des Fehlens einer Kontrollgruppe, welche ausschliesslich eine konventionelle Therapie bekommen hat, nicht zwischen Spontanerholung und dem Behandlungseffekt unterschieden werden. Jedoch wurde die in Studien beschriebene Spontanerholung innerhalb von 20 Wochen nach einem Schlaganfall mit zusätzlichem regelmässigem kardiovaskulärem Training bereits nach 4 Wochen erreicht. Dies ist ein vielversprechendes Resultat im Bereich der kardiovaskulären Rehabilitation nach einem Schlaganfall.

KAPITEL 8 diskutiert die wichtigsten Forschungsergebnisse, präsentiert Einschränkungen und methodische Überlegungen, gibt Empfehlungen für die klinische Praxis und zukünftige Forschung und schliesst mit den wichtigsten Originalbeiträgen der vorliegenden Arbeit ab. Die Implementierung von kardiovaskulärem Training in der Frühphase nach einem Schlaganfall stellt die Rehabilitation vor einzigartige Herausforderungen. Feedback-kontrolliertes, robotergestütztes Laufbandtraining bietet eine Möglichkeit zur Beurteilung der körperlichen Leistungsfähigkeit und zur Einführung von kardiovaskulärer Rehabilitation bei motorisch schwer limitierten Patienten. Das Verfahren fordert grosse Anteile der Körpermuskelmasse, was zu einer zuverlässigen Bewertung der kardiovaskulären Fitness und zu einer signifikant höheren Trainingsintensität im Vergleich zu herkömmlichem, robotergestütztem Laufbandtraining führt. Jedoch wirken sich die Heterogenität der Studienpopulation, allgemeine Probleme in der frühen Rehabilitationsphase und die spontane Erholung negativ auf die Interpretation und die Verallgemeinerung der Ergebnisse aus. Obwohl feedback-kontrolliertes, robotergestütztes Laufbandtraining die Leistungsvorgabe erfolgreich umsetzen konnte, hat das positions-kontrollierte Konzept die schwere Hemiplegie der Probanden nicht berücksichtigt. Weitere Forschung muss sich auf erweiterte Robotersysteme und hoch entwickelte Algorithmen konzentrieren, um die aktive Teilnahme während robotergestützter Aktivität zu fördern. Dies würde zu einer Annäherung an die effektive maximale Leistungsfähigkeit während der kardiopulmonalen Leistungstestung und zur einer besseren Kontrolle der kardiovaskulären Trainingsintensität führen.

Zusammenfassend wird gezeigt, dass subakute Schlaganfallpatienten von kardiovaskulärem Training profitieren und so ihre körperliche Leistungsfähigkeit verbessern. Die Implementierung von robotergestütztem Laufbandtraining wird vorgeschlagen, um die schweren motorischen Einschränkungen zu überwinden und gleichzeitig ein aufgabenspezifisches Training zu fördern. Kardiopulmonale Leistungstestung mittels eines feedback-kontrollierten, robotergestützten Laufbandes zeigte klinische Machbarkeit, gute bis sehr gute Reliabilität und eine akzeptable Wiederholbarkeit, was die wichtigsten kardiopulmonalen Leistungsparameter betrifft. Die Methode ist in der Lage, die Trainingsintensität im Vergleich zu herkömmlichem, robotergestütztem Laufbandtraining deutlich 
zu erhöhen. Beide Ansätze haben in einer 4-wöchigen Interventionsperiode zu einer signifikanten Verbesserung der wichtigsten kardiopulmonalen Leistungsparameter geführt. Erstmals kann eine Methode zur Beurteilung und zum Training der kardiovaskulären Fitness bei motorisch schwer betroffenen Schlaganfallpatienten vorgelegt werden. Diese Erkenntnisse sind ein wichtiger Schritt hin zur Umsetzung und Erforschung der kardiovaskulären Rehabilitation in der Frühphase nach einem Schlaganfall. 


\section{Valorisation}


This dissertation generated new knowledge in the field of cardiovascular rehabilitation early after stroke. In this part of my thesis I would like to present and explain the innovative aspect of the approach, discuss the findings in a social and economic context, reveal the potential for further target groups, and propose approaches for translation into advanced robotic devices and rehabilitation strategies.

\section{Innovation}

Cardiovascular exercise has been shown to be effective in improving exercise capacity in chronic stroke [1]. In this thesis, I was able to provide first evidence that this is also true for subacute stroke survivors [2]. Intensive cardiovascular exercise interventions can be implemented safely in the early stages after stroke. Furthermore, first-ever evidence that cardiovascular fitness can be assessed in severely motor impaired stroke survivors using robotics-assisted technology was presented $[3,4]$ and a method for cardiovascular training in combination with task-specific exercise (i.e. walking) that is of high importance during early post-stroke rehabilitation was proposed [5]. To my knowledge, no other robotics-assisted device has been modified and equipped for cardiovascular rehabilitation early after stroke; therefore, the findings presented here are an innovation.

\section{Social and economic relevance}

Considering the social and economic burden of stroke, effective rehabilitation strategies have high potential to improve recovery and combat the rising costs that have been proposed in several healthcare economic statements $[6,7,8,9]$. The early initiation of exercise training aims to prevent deconditioning and to improve exercise capacity in regard to promote functional independence in daily life. To date, the improvement of cardiovascular fitness after stroke has not been set as a major rehabilitation goal, despite regular cardiovascular exercise having been shown to be able to improve exercise capacity and positively affect a variety of health-related factors [10, 11]. While mildly to moderately impaired individuals after stroke can participate in conventional exercise training programmes, the introduction of cardiovascular exercise in severely impaired stroke survivors presents unique challenges due to various motor limitations. Advanced rehabilitation technology might promote intensive cardiovascular fitness training for severely impaired populations. The improvements in exercise capacity will increase the chance of achieving independence in daily life, which, on a secondary basis, enables social participation and enhances quality of life. The implementation of cardiovascular exercise interventions as early as possible is expected to in- 
crease the functional capacity level of stroke survivors, which might, in turn, decrease the overall expenses during inpatient care and after clinical discharge due to less need of health-care support. In addition, better cardiovascular fitness will decrease the risk for secondary medical complications and enable continuous participation in rehabilitation routines. Fewer adverse events during rehabilitation will lead to better therapy outcomes, and higher functional capacity levels are associated with higher independence during inpatient rehabilitation and after clinical discharge, which will further decrease health-care expenses. This thesis can be taken as a point of departure to further assess these assumptions about improved functional capacity level of stroke survivors on the one hand together with expected lower treatment costs on the other.

\section{Target groups}

The results reported in this dissertation are of importance for several populations suffering from severe motor impairments, which restricts the implementation of effective physical exercise interventions. Although we focussed on stroke due to its large socio-economic impact, the prevention of deconditioning and the improvement of functional capacity might be of interest in a variety of pathologies (e.g. heart failure, brain injury, spinal cord injury, multiple sclerosis, M. Parkinson). Sophisticated robotics-assisted concepts will enable the implementation of cardiopulmonary exercise testing and interpretation after severe medical events (e.g. soon after surgery).

To date, promising results in regard to improved cognitive function and enhanced neuroplasticity after cardiovascular exercise have been published [12, 13, 14, 15, 16]. The technology opens new perspectives in exploring specific effects of early initiation of cardiovascular stress in diseases presenting severe limitation for physical exercise.

The importance of early initiation of cardiovascular exercise and the feasibility using roboticsassisted technologies might also be of importance for the further design and development of advanced robotic concepts. Industries might implement the findings into novel product lines or for supplementary modules. Importantly, engineers and clinicians need to liaise closely.

\section{Translation of knowledge}

While robotic devices have been primarily developed to facilitate motor recovery, a hidden aspect is the potential for cardiovascular rehabilitation in severely disabled populations. The implementation of effective protocols to improve exercise capacity would provide a complement to the application of these devices for neurological rehabilitation, thus expanding the field of application and 
clinical uptake. Our results have revealed the importance of early cardiovascular exercise admission after stroke [2] and demonstrated that feedback-controlled robotics-assisted treadmill exercise (FC-RATE) is a clinically feasible, reliable and repeatable concept for the assessment of exercise capacity in the early stages after severe stroke [4]. Furthermore, FC-RATE has been shown to be able to increase the exercise intensity to almost recommended levels for cardiovascular exercise training [5]. These findings have important implications for further development of assistive technologies and clinical rehabilitation strategies after stroke.

Based on the impact and importance of regular cardiovascular exercise after stroke, we propose further elaborations in control strategies for assisted physical exercise. A major goal will be the introduction of appropriate assist-as-needed strategies or on suitable error augmentation to facilitate cardiovascular stress while considering principles for motor learning. Although we have shown that severely motor impaired individuals can be assessed for cardiovascular fitness in a purely position-controlled path, advanced controllers might be essential to improve the reliability and the validity of cardiopulmonary assessments and increase the exercise intensity up to recommended levels for cardiovascular rehabilitation $[17,18]$.

Our results have led to further promising research activities in robotics-assisted technology. To date, several devices are on the market, which have the potential to provoke cardiovascular stress in severely impaired populations. Our research group is still working on the identification and evaluation of potential novel technology. For example, we recently explored a robotic-assisted stair climbing device [19]. Furthermore, a recent project has been initiated to develop highly specialised cardiopulmonary exercise testing modules for cardiovascular rehabilitation, which will be introduced to the market in the near future.

Although scientific statements reveal the impact of regular cardiovascular exercise training after stroke $[16,17]$ and there is evidence that conventional rehabilitation does not provide appropriate cardiovascular stress to induce training effects [20], the implementation of controlled intervention strategies to improve cardiovascular fitness has not taken place in many rehabilitation institutions. Our findings will, therefore, further emphasise the importance of intensive cardiovascular exercise during stroke rehabilitation. We propose a well-designed and structured cardiovascular rehabilitation strategy that is suitable for inpatient and outpatient settings. Trained medical staff need to categorise stroke survivors based on their cardiovascular and motor status at clinical entry, which enables the further allocation to adequate cardiovascular exercise programmes. Different intervention approaches within groups or on a single basis might then implement regular physical fitness training. While mildly to moderately impaired stroke survivors should perform on conventional devices (e.g. cycle ergometer, treadmill), or preferably participate in outdoor programmes 
(e.g. Nordic walking), rehabilitation centres need to provide total-body recumbent steppers, body weight supported treadmills, robotics-assisted treadmills, or end-effector based gait trainers for the more severely impaired population. Indeed, these conventional methods can be boring due to their monotonous nature. However, further developments will introduce virtual reality setups to enhance motivation, and advanced strategies might shorten the time cost per session through the introduction of interval training methods. Due to the fact that FC-RATE was not able to reach recommended training intensities for cardiovascular rehabilitation, we propose high intensity interval training for further consideration. Controlled bursts of high work rate have shown promising results to improve exercise capacity in subacute stroke [21], and robotics-assisted treadmill technology might to be able to achieve such short periods of peak performance.

\section{Conclusion}

It must be recognised that the improvement of cardiovascular fitness is an important rehabilitation goal after stroke. Based on the work presented in this thesis, it seems justified to strongly propose the implementation of intensive cardiovascular exercise programmes into early stroke rehabilitation. FC-RATE is a novel concept for assessment of cardiovascular fitness and improvement of exercise capacity in severely impaired individuals after stroke. The findings might be highly important in the prevention of deconditioning and the improvement of exercise capacity in a variety of diseases. Further developments in advanced robotic devices and control strategies will lead to better options in guiding cardiovascular exercise intensity, which will improve the application of cardiovascular rehabilitation strategies in severely impaired populations.

The age of technology in rehabilitation sciences has just begun. Rehabilitation robotics are still cost expensive, and elaborate setup structures and complex technical equipment complicate the implementation into clinical practice. It is hoped and expected, however, that the technology will profit from promising research findings that could facilitate an emerging demand, resulting in decreased acquisition costs for end-users. 


\section{References}

[1] M. Y. C. Pang, J. J. Eng, A. S. Dawson, and S. Gylfadottir, "The use of aerobic exercise training in improving aerobic capacity in individuals with stroke: a meta-analysis," Clinical Rehabilitation, vol. 20, no. 2, pp. 97-111, 2006.

[2] O. Stoller, E. D. de Bruin, R. H. Knols, and K. J. Hunt, "Effects of cardiovascular exercise early after stroke: systematic review and meta-analysis," BMC Neurology, vol. 12, no. 1, p. 45, 2012.

[3] O. Stoller, M. Schindelholz, L. Bichsel, C. Schuster, R. A. de Bie, E. D. de Bruin, and K. J. Hunt, "Feedbackcontrolled robotics-assisted treadmill exercise to assess and influence aerobic capacity early after stroke: a proofof-concept study," Disabil Rehabil Assist Technol, vol. 9, no. 4, pp. 271-278, 2014.

[4] O. Stoller, E. D. de Bruin, M. Schindelholz, C. Schuster-Amft, R. A. de Bie, and K. J. Hunt, "Cardiopulmonary exercise testing early after stroke using feedback-controlled robotics-assisted treadmill exercise: test-retest reliability and repeatability," Journal of Neuroengineering and Rehabilitation, vol. 11, p. 145, 2014.

[5] O. Stoller, E. D. de Bruin, M. Schindelholz, C. Schuster-Amft, R. A. de Bie, and K. J. Hunt, "Efficacy of feedback-controlled robotics-assissted treadmill exercise to improve cardiovascular fitness early after stroke: a randomized controlled pilot trial," Journal of Neurologic Physical Therapy, submitted.

[6] A. Di Carlo, "Human and economic burden of stroke," Age and Ageing, vol. 38, no. 1, pp. 4-5, 2009.

[7] M. Kaste, R. Fogelholm, and A. Rissanen, "Economic burden of stroke and the evaluation of new therapies,' Public Health, vol. 112, no. 2, pp. 103-12, 1998.

[8] J. Leal, R. Luengo-Fernandez, A. Gray, S. Petersen, and M. Rayner, "Economic burden of cardiovascular diseases in the enlarged european union," Eur Heart J, vol. 27, no. 13, pp. 1610-9, 2006.

[9] P. Youman, K. Wilson, F. Harraf, and L. Kalra, “The economic burden of stroke in the united kingdom," Pharmacoeconomics, vol. 21 Suppl 1, pp. 43-50, 2003.

[10] N. Cavill, S. Kahlmeier, and F. Racioppi, Physical activity and health in Europe: evidence for action. World Health Organization, 2006.

[11] W. L. Haskell, I. M. Lee, R. R. Pate, K. E. Powell, S. N. Blair, B. A. Franklin, C. A. Macera, G. W. Heath, P. D. Thompson, and A. Bauman, "Physical activity and public health - updated recommendation for adults from the american college of sports medicine and the american heart association," Circulation, vol. 116, no. 9, pp. 1081-1093, 2007

[12] S. J. Colcombe, A. F. Kramer, K. I. Erickson, P. Scalf, E. McAuley, N. J. Cohen, A. Webb, G. J. Jerome, D. X. Marquez, and S. Elavsky, "Cardiovascular fitness, cortical plasticity, and aging," Proc Natl Acad Sci U S A, vol. 101, no. 9, pp. 3316-21, 2004.

[13] M. S. El-Tamawy, F. Abd-Allah, S. M. Ahmed, M. H. Darwish, and H. A. Khalifa, "Aerobic exercises enhance cognitive functions and brain derived neurotrophic factor in ischemic stroke patients," NeuroRehabilitation, vol. 34, no. 1, pp. 209-13, 2014.

[14] P. J. Smith, J. A. Blumenthal, B. M. Hoffman, H. Cooper, T. A. Strauman, K. Welsh-Bohmer, J. N. Browndyke, and A. Sherwood, "Aerobic exercise and neurocognitive performance: a meta-analytic review of randomized controlled trials," Psychosom Med, vol. 72, no. 3, pp. 239-52, 2010.

[15] K. Knaepen, M. Goekint, E. M. Heyman, and R. Meeusen, "Neuroplasticity - exercise-induced response of peripheral brain-derived neurotrophic factor: a systematic review of experimental studies in human subjects," Sports Med, vol. 40, no. 9, pp. 765-801, 2010.

[16] F. M. Ivey, A. S. Ryan, C. E. Hafer-Macko, and R. F. Macko, "Improved cerebral vasomotor reactivity after exercise training in hemiparetic stroke survivors," Stroke, vol. 42, no. 7, pp. 1994-2000, 2011 
[17] N. F. Gordon, M. Gulanick, F. Costa, G. Fletcher, B. A. Franklin, E. J. Roth, and T. Shephard, "Physical activity and exercise recommendations for stroke survivors - an american heart association scientific statement from the council on clinical cardiology, subcommittee on exercise, cardiac rehabilitation, and prevention; the council on cardiovascular nursing; the council on nutrition, physical activity, and metabolism; and the stroke council," Stroke, vol. 35, no. 5, pp. 1230-1240, 2004.

[18] S. A. Billinger, R. Arena, J. Bernhardt, J. J. Eng, B. A. Franklin, C. M. Johnson, M. MacKay-Lyons, R. F. Macko, G. E. Mead, E. J. Roth, M. Shaughnessy, A. Tang, C. American Heart Association Stroke, C. Council on, N. Stroke, L. Council on, H. Cardiometabolic, E. Council on, Prevention, and C. Council on Clinical, "Physical activity and exercise recommendations for stroke survivors: a statement for healthcare professionals from the american heart association/american stroke association,” Stroke, vol. 45, no. 8, pp. 2532-53, 2014.

[19] O. Stoller, M. Schindelholz, L. Bichsel, and K. J. Hunt, "Cardiopulmonary responses to robotic end-effectorbased walking and stair climbing," Medical Engineering and Physics, vol. 36, no. 4, pp. 425-31, 2014.

[20] M. J. MacKay-Lyons and L. Makrides, "Cardiovascular stress during a contemporary stroke rehabilitation program: Is the intensity adequate to induce a training effect?," Archives of Physical Medicine and Rehabilitation, vol. 83, no. 10, pp. 1378-1383, 2002.

[21] P. Boyne, K. Dunning, D. Carl, M. Gerson, J. Khoury, and B. Kissela, "High-intensity interval training in stroke rehabilitation," Top Stroke Rehabil, vol. 20, no. 4, pp. 317-30, 2013. 

Acknowledgment 
The success of any project depends largely on the encouragement and guidance from a group of people. I take this opportunity to express my appreciation and thanks to all those who have been involved in this research project.

Prof. dr. Kenneth J. Hunt, my local supervisor, is an outstanding engineer and a wise character. You have been a tremendous mentor to me. I would like to thank you for giving me the opportunity to work on such an interesting project, encouraging me during the entire process, giving professional and personal advice, and for allowing me to grow as a scientist but also as a human being. Your advice on both research as well as on my personality have been priceless.

Prof. dr. Rob A. de Bie, my main supervisor, is a leader in rehabilitation research with a remarkable personality. I would like to thank you for guiding me through the $\mathrm{PhD}$ process as an external candidate at CAPHRI, giving me the opportunity to become an independent researcher, and encouraging me to think further. It was a pleasure being a $\mathrm{PhD}$ candidate in your research programme.

PD dr. Eling D. de Bruin, my independent supervisor, is an experienced and straightforward researcher with an outstanding methodological know-how. I would like to thank you for your scientific and personal advice throughout the entire process, and especially for your open ear and friendliness during difficult phases.

The Institute for Rehabilitation and Performance Technology at Bern University of Applied Sciences, my employer, is a centre of excellence for biomedical engineering in the field of rehabilitation technology. I would like to thank Matthias Schindelholz for his professional technical support, his reliability, and his honest personality. I also want to thank the entire academic staff for their valuable support, especially Lukas Bichsel, Marco Laubacher, Christian Dietrich, Jittima Saengsuwan, and Michael Muster. Special thanks go to Dr. Franz Baumberger: I appreciate your confidence and constant support.

The Reha Rheinfelden, our clinical cooperation partner, is a first-class rehabilitation centre in Northwestern Switzerland. I would like to thank Prof. Dr. Thierry Ettlin and Dr. Corina SchusterAmft for the opportunity to perform experimental clinical research in their excellent facilities. I appreciate your faith in me. I especially would like to thank Heike Rosemeyer for her continuous support during clinical experimentation. Thank you for your critical inputs, your attention and support during the recruitment, and for your accurate work. I would also like to thank the clinical staff involved for their professional support, especially Dr. Niklaus Urscheler, Nadine Springinsfeld, Rahel Peyer, Daniel Vosseler, Michael McCaskey, Andrea Henneke, Heike Rösner, Doris Felber, Willi Bäckert, and Zorica Suica. 
The "Herzpraxis am Rhein" is a cardiac private practice in Rheinfelden. I would like to thank Dr. Beat Spoendlin for his professional advice on the cardiac aspects of this project, and for the cardiopulmonary resuscitation courses. Also, many thanks to Dr. Andreas Rohner and Dr. Marco Kummer for the cardiac screening of the patients.

To all the participants that were involved in clinical research and whose interest stimulated them to participate in an unknown area of research: many thanks for your motivation, your efforts, and your confidence in our knowledge and in the technology.

I would also like to thank the members of the assessment committee and the corona for their time, interest, and valuable feedback: Prof. dr. Martin H. Prins, Prof. dr. Roger Gassert, Dr. Kenneth Meijer, Prof. dr. Robert Riener, and Prof. dr. Lodewijk W. van Rhijn.

I am very grateful for the scientific, personal, and even spiritual input and support of many people. A special thank goes to my paranymphs and classmates Corinne Ammann-Reiffer and Slavko Rogan. I would also like to thank Dr. Carolien Bastiaenen, Dr. Judith Sieben, and all my $\mathrm{PhD}$ classmates for their commitment.

Life consists of science and love. Words cannot express how grateful I am to have such wonderful people behind me. Many thanks to all my family members for their continual support, and to all of my friends who encouraged me to strive towards my goal and helped me to clear my mind.

Finally, I would like to thank my wonderful wife Sabina. This work would not have been possible without you. Thank you for your patience and for your unconditional love. 

About the author 


\section{Curriculum vitae}

Oliver Stoller was born on 29 August 1980 in Frauenfeld, Switzerland. He received a B.Sc. in Physical Therapy from the University College Physiotherapy Thim van der Laan (Switzerland / the Netherlands) in 2005, and a M.Sc. in Physical Therapy from the University College Physiotherapy Thim van der Laan (Switzerland / the Netherlands) in cooperation with the Technical University Lisbon (Portugal) and the Free University of Brussels (Belgium) in 2007. Oliver Stoller gained practical experience in several rehabilitation centres before he was appointed research assistant and lecturer at the Health Department of the Bern University of Applied Sciences (Switzerland). In 2010, he was then admitted as a PhD candidate at the CAPHRI Research School, in the Department of Epidemiology of the Maastricht University (the Netherlands), and as a research associate at the Institute for Rehabilitation and Performance Technology in the Department of Engineering and Information Technology of the Bern University of Applied Sciences (Switzerland).

Oliver Stoller's research interests focus on human-machine interaction, biological signal processing and advanced assessment tools to promote physical activity and motor learning. He is convinced that specific exercise concepts will become one of the more important areas in future health care management, with the connection of technical devices to the human body as a major trend.

Oliver Stoller received an Early Postdoc Mobility fellowship from the Swiss National Science Foundation in 2014 to follow up on relevant questions in the field of biomedical engineering, rehabilitation and sports sciences. 


\section{Publications}

\section{Journal articles (peer reviewed)}

O. Stoller, E.D. de Bruin, M. Schindelholz, C. Schuster-Amft, R.A. de Bie, K.J. Hunt, "Efficacy of feedbackcontrolled robotics-assisted treadmill exercise to improve cardiovascular fitness early after stroke: a randomized controlled pilot trial," Journal of Neurologic Physical Therapy, submitted - in revision.

O. Stoller, E.D. de Bruin, M. Schindelholz, C. Schuster-Amft, R.A. de Bie, K.J. Hunt, "Cardiopulmonary exercise testing early after stroke using feedback-controlled robotics-assisted treadmill exercise: test-retest reliability and repeatability," Journal of Neuroengineering and Rehabilitation, vol 11, p. 145, 2014.

O. Stoller, M. Schindelholz, L. Bichsel, K.J. Hunt, "Cardiopulmonary responses to robotic end-effector-based walking and stair climbing," Medical Engineering and Physics, vol. 36, no. 4, pp. 425-431, 2014.

M. Schindelholz, O. Stoller, K.J. Hunt, "A software module for cardiovascular rehabilitation in robotics-assisted treadmill exercise," Biomedical Signal Processing and Control, vol. 10, pp. 296-307, 2014.

O. Stoller, E.D. de Bruin, M. Schindelholz, C. Schuster-Amft, R.A. de Bie, K.J. Hunt, "Cardiovascular rehabilitation soon after stroke using feedback-controlled robotics-assisted treadmill exercise: study protocol of a randomised controlled pilot trial," Trials, vol. 14, p. 304, 2013.

O. Stoller, M. Schindelholz, L. Bichsel, C. Schuster, R.A. de Bie, E.D. de Bruin, K.J. Hunt, "Feedback-controlled robotics-assisted treadmill exercise to assess and influence aerobic capacity early after stroke: a proof-of-concept study," Disability and Rehabilitation: Assistive Technology, vol. 9, no. 4, pp. 271-278, 2014.

O. Stoller, E.D. de Bruin, M. Schindelholz, C. Schuster, R.A. de Bie, K.J. Hunt, "Evaluation of exercise capacity after severe stroke using robotics-assisted treadmill exercise: a proof-of-concept study," Technology and Health Care, vol. 21, no. 2, pp. 157-166, 2013.

O. Stoller, E.D. de Bruin, R.H. Knols, K.J. Hunt, "Effects of cardiovascular exercise early after stroke: systematic review and meta-analysis," BMC Neurology, vol. 12, no. 1, p. 45, 2012.

O. Stoller, M. Waser, L. Stammler, C. Schuster, 'Evaluation of robot-assisted gait training using integrated biofeedback in neurologic disorders," Gait and Posture, vol. 35, no. 4, pp. 595-600, 2012.

\section{Conference proceedings}

O. Stoller, E.D. de Bruin, C. Schuster-Amft, M. Schindelholz, R.A. de Bie, K.J. Hunt, "Feedback-controlled roboticsassisted treadmill exercise for cardiovascular training and assessment early after severe stroke," European Stroke Conference, Vienna, Austria, May 2015.

O. Stoller, E.D. de Bruin, C. Schuster-Amft, M. Schindelholz, R.A. de Bie, K.J. Hunt, "Feedback-controlled roboticsassisted treadmill exercise for cardiovascular rehabilitation in severely motor impaired individuals early after stroke," Summer School on Neurorehabilitation, Baiona, Spain, September 2014. 
O. Stoller, E.D. de Bruin, C. Schuster-Amft, M. Schindelholz, R.A. de Bie, K.J. Hunt, 'Feedback-controlled roboticsassisted treadmill exercise for cardiovascular rehabilitation in severely motor impaired individuals early after stroke,' Swiss Physiotherapy Congress, Bern, Switzerland, June 2014.

O. Stoller, E.D. de Bruin, R.H. Knols, K.J. Hunt, "Effects of cardiovascular exercise early after stroke: systematic review and meta-analysis," World Congress of Neurorehabilitation, Melbourne, Australia, May 2012.

O. Stoller, M. Schindelholz, L. Bichsel, C. Schuster, R.A. de Bie, E.D. de Bruin, K.J. Hunt, ’Exercise testing early after stroke using feedback-controlled robotics-assisted treadmill exercise," World Congress of Neurorehabilitation, Melbourne, Australia, May 2012.

O. Stoller, M. Waser, L. Stammler, C. Schuster, "Evaluation of robot-assisted gait training using integrated biofeedback in neurologic disorders," World Congress of Neurorehabilitation, Melbourne, Australia, May 2012.

O. Stoller, E.D. de Bruin, R.H. Knols, K.J. Hunt, ”Effects of cardiovascular exercise early after stroke: systematic review and meta-analysis," Swiss Physiotherapy Congress, Geneva, Switzerland, May 2012.

O. Stoller, M. Schindelholz, L. Bichsel, C. Schuster, R.A. de Bie, E.D. de Bruin, K.J. Hunt, ’Exercise testing early after stroke using feedback-controlled robotics-assisted treadmill exercise," Swiss Physiotherapy Congress, Geneva, Switzerland, May 2012.

O. Stoller, M. Waser, L. Stammler, C. Schuster, "Evaluation of robot-assisted gait training using integrated biofeedback in neurologic disorders," International Neurorehabilitation Symposium, Zurich, Switzerland, June/July 2011.

O. Stoller, M. Waser, L. Stammler, C. Schuster, "Evaluation of robot-assisted gait training using integrated biofeedback in neurologic disorders," World Congress of Physical Therapy, Amsterdam, Netherlands, June 2011. 
